\author{
Universidade de São Paulo \\ Instituto de Física
}

\title{
Transições de fase em modelos estocásticos para descrever epidemias
}

\author{
David Rodrigues de Souza
}

Orientadora: Prof ${ }^{\mathrm{a}}$. Dr ${ }^{\mathrm{a}}$. Tânia Tomé Martins de Castro

Tese de doutorado apresentada ao Instituto de Física para a obtenção do título de Doutor em Ciências

Banca examinadora:

Prof $^{\mathrm{a}}$. Dr ${ }^{\mathrm{a}}$. Tânia Tomé Martins de Castro (IFUSP)

Prof. Dr. André de Pinho Vieira (IFUSP)

Prof. Dr. José Roberto Drugowich de Felício (FFCLRP-USP)

Prof. Dr. Marcelo Lobato Martins (UFV)

Prof. Dr. Ronald Dickman (UFMG)

São Paulo

2012 
FICHA CATALOGRÁFICA

Preparada pelo Serviço de Biblioteca e Informação do Instituto de Física da Universidade de São Paulo

Souza, David Rodrigues de

Transições de fase em modelos estocásticos para descrever epidemias. - São Paulo, 2012.

Tese (Doutorado) - Universidade de São Paulo. Instituto de Física, Depto. Física Geral.

Orientador: Prof ${ }^{\mathrm{a}} \mathrm{Dr}{ }^{\mathrm{a}}$ Tânia Tomé Martins de Castro

Área de Concentração: Física

Unitermos: 1.Mecânica estatística; 2. Modelos epidemiológicos; 3 . Processos estocásticos; 4. Física biológica; 5 . Transições de fase em sistemas irreversiveis.

USP/IF/SBI-072/2012 


\section{Agradecimentos}

À minha orientadora, $\operatorname{Prof}^{\mathrm{a}} \operatorname{Dr}^{\mathrm{a}}$ Tânia Tomé, pela oportunidade, pelos ensinamentos, pelo tempo e pela paciência dedicada para o desenvolvimento deste trabalho.

Aos colaboradores deste trabalho, Robert M. Ziff, Suani Pinho e Florisneide Barreto, pelas hipóteses e sugestões.

Ao Prof. Dr. Mário J. Oliveira, pela colaboração em todas as etapas deste trabalho, levantando críticas e interpretações enriquecedoras.

Aos colegas, pela fraternidade e pelo companherismo sob qualquer estado de humor. Agradecimento especial ao Lucas, Maicon, Áttila, Rafael e Oscar, pelas dicas de programação e manutenção dos computadores.

Aos funcionários do Instituto, por propiciar boas condições de trabalho. Aos colegas do CCA-UFES pela flexibilização do meu horário de trabalho. A CAPES e ao CNPq, pelo financiamento parcial deste trabalho.

À minha família, pelo apoio, pelos valores e pelo subsídio ao meu desenvolvimento. 


\section{Resumo}

Este trabalho busca descrever sistemas irreversíveis (aqueles que não obedecem ao balanceamento detalhado) usando o formalismo mecânico-estatístico que tem como base a dinâmica estocástica. Nossos principais objetivos são: (i) a investigação do comportamento crítico e das possíveis classes de universalidade em sistemas irreversíveis; (ii) a modelagem da dinâmica de propagação de epidemias. Primeiramente investigamos o modelo suscetívelinfectado-recuperado (SIR) estocástico e espacialmente estruturado. Nesse modelo, os indivíduos são divididos em três classes: suscetível (S), infectado (I) e recuperado (R). Um indivíduo suscetível pode tornar-se infectado devido ao contato com um vizinho infectado, e um indivíduo infectado pode recuperar-se espontaneamente. Este modelo exibe transição de fase entre uma fase em que a doença se espalha e uma fase em que não há espalhamento da doença. Tratando cada par suscetível-infectado como uma conexão através da qual pode haver propagação da epidemia, mostramos que é possível estabelecer uma conexão entre o modelo SIR e o modelo de percolação. Assim, pudemos utilizar métodos da teoria de percolação usual para determinar o limiar de espalhamento epidêmico. Por meio de aproximações de campo médio dinâmico, simulações computacionais de Monte Carlo estacionárias e simulações dependentes do tempo, determinamos o ponto crítico e o comportamento crítico desse modelo. Ademais, propomos dois modelos para descrever um processo epidêmico de transmissão vetorial. Consideramos duas populações interagentes, uma formada por vetores e a outra por hospedeiros. Os vetores podem ser suscetíveis (S) ou infectados (I), enquanto os estados permitidos para os hospedeiros são os mesmos do modelo SIR. O processo de transmissão da doença ocorre devido ao contato local de um hospedeiro (vetor) suscetível com um vetor (hospedeiro) infectado. Determinamos o limiar de infecção, o tamanho da epidemia e mostramos que ambos os modelos exibem transições de fase de segunda ordem e que pertencem à classe de universalidade da percolação dinâmica isotrópica. 


\section{Abstract}

This study aims to describe irreversible systems (those that do not obey detailed balance) using a statistical mechanics formalism based on stochastic dynamics. Our main objectives are: (i) to investigate the critical behavior and the possible universality classes in irreversible systems; (ii) and modeling the dynamics of the epidemic spreading. First we investigate the stochastic and spatially structured susceptible-infected-recovered model (SIR). In this model, individuals are divided into three classes: susceptible (S), infected (I) and recovered (R). A susceptible individual may become infected due to contact with an infected neighbor, and an infected individual may recover spontaneously. This model exhibits a phase transition between a phase in which the epidemic spreads and a phase where there is no spreading of the disease. Treating each susceptible-infected pair as a connection through which there may be epidemic spreading, we show that it is possible to establish a connection between the SIR model and the percolation model. Thus we are able to use methods of the theory of standard percolation for determining the epidemic spreading. By means of dynamic mean-field approximations and stationary and time-dependent computational Monte Carlo simulations, we determine the critical point and critical behavior of this model. In addition, we propose two models to describe the vector transmitted epidemic process. We consider two interacting populations, one formed by vectors and other by hosts. The vectors may be susceptible (S) or infected (I), where the states allowed for the hosts are the same as those in the SIR model. Transmission of the disease occurs due to contact between a local host (vector) and a susceptible vector (host) infected. We determine the threshold of infection, the size of the epidemic, and show that both models exhibit second order phase transitions that belong to the universality class of dynamic isotropic percolation. 


\section{Sumário}

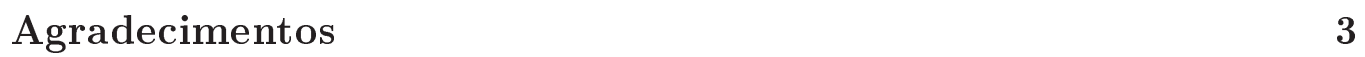

Resumo 5

\begin{tabular}{ll}
\hline Abstract & 7
\end{tabular}

$\begin{array}{lll}1 & \text { Introducão } & 15\end{array}$

2 Modelo de percolação 19

2.1 Introducão . . . . . . . . . . . . . . . . . . . . . . . . . . . . . 19

2.2 Solucão exata em uma dimensão . . . . . . . . . . . . . . . . . 21

2.3 Extensão para redes d-dimensionais . . . . . . . . . . . . . 24

2.4 Solucão exata na árvore de Cavlev. . . . . . . . . . . . . . . 26

2.5 Comportamento crítico na árvore de Cavlev . . . . . . . . . . 29

2.6 Comportamento crítico . . . . . . . . . . . . . . . . . 30

$3 \quad$ Modelo Suscetível-Infectado-Recuperado (SIR) 35

3.1 Descricão do Modelo . . . . . . . . . . . . . . . . . . . . . 35

3.2 Equacão mestra para o modelo SIR . . . . . . . . . . . . . 37

3.3 Aproximacão de Campo Médio Simples . . . . . . . . . . . . . 40

3.4 Aproximacão de Campo Médio por Pares . . . . . . . . . . . . 44

3.5 Simulacões de Monte Carlo estacionárias . . . . . . . . . . . . 50

3.6 Simulacões na rede quadrada . . . . . . . . . . . . . . . . . 52

3.7 Comportamento crítico . . . . . . . . . . . . . . . 56

3.8 Simulacỗes na rede triangular . . . . . . . . . . . . . . 63

\begin{tabular}{|ll}
4 Modelo de infeccão cruzada I & 67
\end{tabular}

4.1 Descricão do modelo . . . . . . . . . . . . . . . . . . . 67

4.2 Equacão mestra para o modelo . . . . . . . . . . . . . . 71

4.3 Aproximacão de campo médio simples . . . . . . . . . . . . 74

4.4 Aproximacão de campo médio por pares . . . . . . . . . . . . 78

4.5 Simulacôos computacionais estacionárias . . . . . . . . . . . . . 84 
4.6 Simulacões dependentes do tempo . . . . . . . . . . . . . . . 95

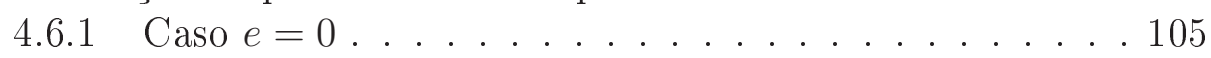

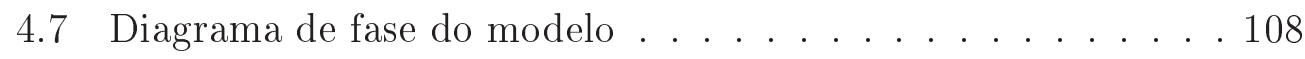

5 Modelo de infeccão cruzada II 111

5.1 Descricão do modelo . . . . . . . . . . . . . . . . . . 111

5.2 Equacão mestra . . . . . . . . . . . . . . . . . . . . . . . 113

5.3 Aproximacão de campo médio simples . . . . . . . . . . . . 114

5.4 Aproximacão de campo médio por pares . . . . . . . . . . 117

5.5 Simulacões estacionárias . . . . . . . . . . . . . . . . . . . 120

5.6 Expoentes críticos estáticos . . . . . . . . . . . . . . . . . 128

5.7 Simulações dependentes do tempo . . . . . . . . . . . . . . . . 131

6 Comparacões e aplicacões dos modelos 137

6.1 Comparacão entre os modelos . . . . . . . . . . . . . . . . . 138

6.2 Aplicação do modelo I . . . . . . . . . . . . . . . . . . . 145

\begin{tabular}{lll}
\hline 7 & Conclusões & 147
\end{tabular} 


\section{Lista de Figuras}

2.1 Redes de tamanho $L=32$ para diferentes probabilidades $p$. . 20

2.2 Exemplo de árvore de Cavlev com $z=3$. . . . . . . . . 27

3.1 Esboco das transicões entre os estados para o modelo SIR . . 36

3.2 Curvas de evolucão das densidades $\operatorname{com} c=0,25$. . . . . . . . 42

3.3 Densidade de recuperados versus d . . . . . . . . . . . . . . . 43

3.4 Curvas de evolucão das densidades $\operatorname{com} c=0,25$. . . . . . . . 49

3.5 Densidade de recuperados versus c . . . . . . . . . . . . 49

3.6 Retratos da rede com $L=100$ e $c=0,12 \ldots$. . . . . . . . . 53

3.7 Retratos da rede com $L=100$ e $c=0.15$. . . . . . . . . . . 53

3.8 Retratos da rede com $L=100$ e $c=0.1765$. . . . . . . . . 54

3.9 Retratos da rede com $L=100$ e $c=0.19$. . . . . . . . . . 54

3.10 Densidade de indivíduos recuperados versus d . . . . . . . . 55

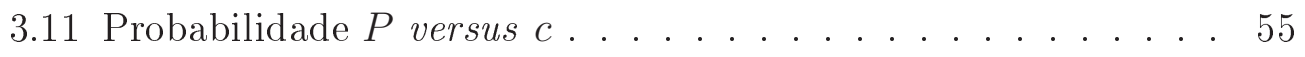

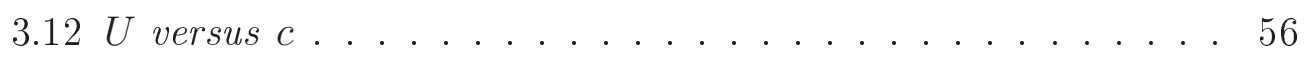

3.13 UP versus d . . . . . . . . . . . . . . . . . . . . . . 58

3.14 UP versus $1 / L \ldots \ldots \ldots$

3.15 Densidade de recuperados versus $L$. . . . . . . . . . . . 60

$3.16 \operatorname{Ln}(P)$ versus $\ln (L) \ldots \ldots \ldots$

$3.17 \operatorname{Ln}(S)$ versus $\operatorname{Ln}(L) \ldots \ldots \ldots \ldots$. . . . . . . . . . . 61

3.18 Colapso de dados de $P$ para diferentes valores de $L$. . . . . . 61

3.19 Colapso de dados de $S$ para diferentes valores de $L$. . . . . . 62

3.20 Colapso de dados de UP para diferentes valores de $L$. . . . . 62

3.21 Estrutura da rede triangular . . . . . . . . . . . . . . . . 63

3.22 grandezas estacionárias para rede triangular . . . . . . . . . . 64

$3.23 P(>s . c) s^{\tau-2}$ versus $s^{\sigma} \ldots \ldots \ldots \ldots \ldots$. . . . . . . . . . . . . . . . 66

3.24 Coeficientes de $P(>s, c) s^{\tau-2}$ versus $\ldots . . . . . .666$

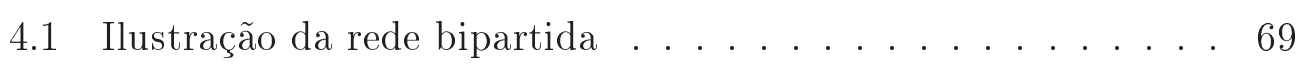

4.2 Regras de transicões locais para o modelo . . . . . . . . . . . 69

4.3 Diagrama de fase . . . . . . . . . . . . . . 76 
4.4 Evolucão da densidade de infectados . . . . . . . . . . . . 77

4.5 Diagrama de fase do modelo . . . . . . . . . . . . . . . . . . 82

4.6 Densidade de vetores e hospedeiros infectados . . . . . . . . . 83

4.7 Densidade de hospedeiros recuperados $\left(H_{r}\right)$ versus $a^{\prime} \ldots$. . . 89

4.8 P versus $a^{\prime} \ldots \ldots \ldots$. . . . . . . . . . . . . . . . . . . . . . . . . . 90

4.9 Cumulante $U$ versus $a$. . . . . . . . . . . . . . . . . . . . . . . 91

4.10 Produto $U P$ versus $a^{\prime} \ldots \ldots$. . . . . . . . . . . . . . . . . . . . 92

4.11 Densidade probabilidade $P$ e cumulante para $e^{\prime}=0.100 \ldots 93$

4.12 Densidade probabilidade $P$ e cumulante para $e^{\prime}=0.400$. . . 94

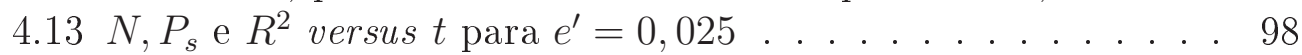

4.14 Expoentes dinâmicos versus $1 / t$ para $e^{\prime}=0.025$. . . . . . . . 100

4.15 Expoentes dinâmicos versus $1 / t$ para $e^{\prime}=0,100 \ldots . . . . .104$

$4.16 N(t)$ e $R^{2}(t)$ versus $t$ para $e=0 \ldots \ldots$. . . . . . . . . . 106

4.17 Expoentes dinâmicos versus $1 / t$ para $e=0 \ldots$. . . . . . . 107

4.18 Diagrama de fase no plano a versus e . . . . . . . . . . . . . . 109

4.19 Diagrama de fase no plano e/c versus a/d . . . . . . . . . . 109

5.1 Regras de transicão para o modelo . . . . . . . . . . . . . . 112

5.2 Densidade de infectados em funcão do tempo . . . . . . . . . . 115

5.3 Diagrama de fase do modelo na aproximação de campo médio 119

5.4 Retratos da evolucão temporal . . . . . . . . . . . . . . . 122

5.5 Grandezas estacionárias versus a para $e=r=0,100 \quad \ldots 124$

$5.6 \quad U P$ próximo do ponto crítico . . . . . . . . . . . . . . . . 125

$5.7 \quad$ Grandezas estacionárias versus a para $e=0.025$. . . . . . . . 126

5.8 Grandezas estacionárias versus a para $e=0.200$. . . . . . . . 127

$5.9 \log (\mathrm{P}) \operatorname{versuslog}(\mathrm{L}) \quad \ldots \ldots \ldots$

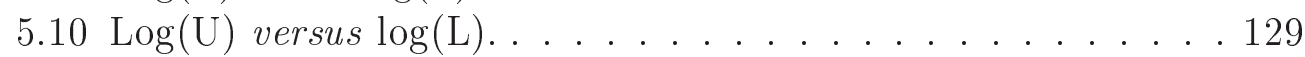

5.11 Grandeza UP versus $1 / L$. . . . . . . . . . . . . . . . . . . 130

$5.12 P_{>s} s^{\tau-2}$ versus $s^{\sigma} \ldots \ldots \ldots \ldots$

5.13 Coeficiente linear $K_{2}$ versus a . . . . . . . . . . . . . . 131

5.14 Grandezas médias em função do tempo . . . . . . . . . . . . . 133

5.15 Expoentes dinâmicos . . . . . . . . . . . . . . . . . . 134

5.16 Diagrama de fase no plano $a-e$. . . . . . . . . . . . 135

6.1 Regras do modelo I . . . . . . . . . . . . . . . . . . . 138

6.2 Regras do modelo II . . . . . . . . . . . . . . . . . . . . . . . . 139

6.3 Diagrama de fase no plano $a-e$. . . . . . . . . . . . . . . . . . 141

6.4 Diagrama de fase no plano $a-E$. . . . . . . . . . . . . . . 142

6.5 Diagrama de fase no plano e/c-a/d . . . . . . . . . . . . 142

6.6 Linha de controle vetorial . . . . . . . . . . . . . . . . . . . . 144

6.7 Epidemia de dengue em Salvador 1995 . . . . . . . . . . . . . 146 


\section{Lista de Tabelas}

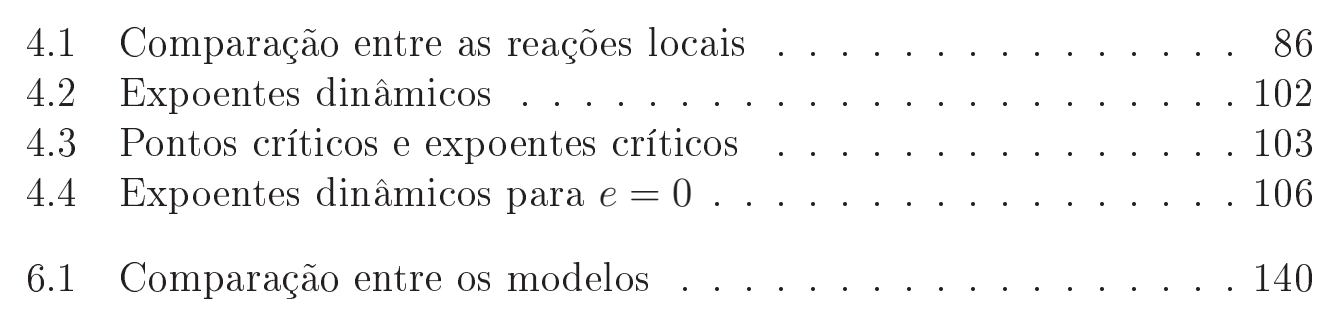




\section{Capítulo 1}

\section{Introdução}

A descrição do espalhamento de uma epidemia em uma população envolve dois níveis de interação [1] []: um que denominaremos microscópico, referente às interações entre o agente causador da doença (vírus, bactéria ou verme) no vetor e no hospedeiro e, em alguns casos, considerando também o ciclo de vida do vetor; outro que denominaremos de nível macroscópico, em que é importante analisar as interações entre os indivíduos infectados e suscetíveis e buscar entender os aspectos gerais necessários de transmissão da doença.

$\mathrm{Na}$ modelagem da propagação de epidemias considerando o caso macroscópico, pode-se fazer uma descrição determinística [1,7, 9] ou estocástica [1-4, 10-14]. A descrição determinística é usualmente feita a partir de equações diferenciais, similares às equações da cinética química, nas quais o número de indivíduos infectados aumenta proporcionalmente à quantidade de indivíduos suscetíveis.

Na abordagem estocástica, considera-se que os indivíduos suscetíveis, infectados e recuperados (quando for o caso) podem aumentar ou diminuir devido às transições de um estado para outro.

O modelo epidêmico conhecido como suscetível-infectado-recuperado (SIR), introduzido por Kermack e McKendrick em 1927 [15], busca a descrição determinística do espalhamento de uma doença na qual os indivíduos infectados podem se recuperar, e se tornar permanentemente imunes. Neste modelo, cada indivíduo pode estar em uma das três classes: suscetível (S), infectado (I) ou recuperado/imune/morto (R). O processo de infecção é catalítico, ou seja, a população de infectados cresce proporcionalmente ao número de infectados, e o processo de imunização é espontâneo. Assim, indivíduos infectados podem espontaneamente tornar-se recuperados, ficando permanentemente imunes.

As hipóteses implícitas no modelo SIR são: o tempo de vida do hos- 
pedeiro é muito maior que o tempo de duração da epidemia (tal que durante a epidemia a população pode ser considerada constante); o processo de recuperação proporciona imunidade permanente ao indivíduo em relação à doença. Essas hipóteses são válidas para várias doenças [2, 3, 7, 14, 16 20] e, assim, mesmo sem considerar os efeitos da estrutura espacial e da estocasticidade, é possível obter curvas epidêmicas adequadas para muitas epidemias. Além disso, o modelo pode ser usado como ferramenta de previsão do total de indivíduos de certa população que deve ser imunizado por meio de vacinação para prevenir epidemias [9, 21 27], ou mesmo servir de parâmetro para definição de políticas públicas para controle e gerenciamento de doenças infectocontagiosas [16, 28, 30].

O modelo SIR é um modelo simples que pode prever um limiar de infecção acima do qual a epidemia se espalha e abaixo do qual não há propagação da doença. Devido ao fato de ser definido por um processo local que segue o ciclo: $(S \rightarrow I \rightarrow R)$, as reações $S \rightarrow I$ e $I \rightarrow R$ ocorrem com certas taxas, mas as reações reversas são expressamente proibidas e, assim, o sistema é irreversível e não obedece ao balanceamento detalhado [12, 31, 32].

Versões estocásticas deste modelo podem ser do tipo "nascimento e morte" [1,33,34, nas quais se consideram equações mestras em que o número de indivíduos de cada espécie são as variáveis estocásticas. Apesar do nome, "nascimento e morte" não significa natalidade ou mortalidade de indivíduos e diz respeito somente ao aumento do número de infectados (que ocorre devido ao decréscimo do número de indivíduos suscetíveis) e ao decaimento de infectados (devido ao processo de recuperação com imunização permanente). Uma das primeiras descrições do sistema SIR por meio de um processo estocástico do tipo "nascimento e morte" é a dada por Bailey [10,11.

Para uma análise mais detalhada e completa do processo de dispersão da doença, deve-se levar em consideração a estrutura espacial [35, 36] e a individualidade de cada elemento da população. Nesse caso, consideramos uma rede onde cada nó ou sítio representa um único indivíduo ao qual associamos uma variável estocástica para denotar seu estado de saúde com relação a certa doença. Além disso, nesse tratamento os processos de infecção e recuperação são estocásticos e locais (envolvem somente os vizinhos mais próximos), e o processo SIR é tratado como um modelo estocástico e espacialmente estruturado. A descrição por meio de um processo estocástico do tipo "nascimento e morte" (citado acima) pode ser obtida a partir do modelo SIR estocástico e espacialmente estruturado por meio de uma redução de graus de liberdade [34.

Por meio de simulações de Monte Carlo estacionárias e dependentes do tempo, podemos investigar grandezas relevantes, tais como o raio de giração e número médio de infectados; e determinar o ponto crítico (limiar de 
espalhamento epidêmico) e os expoentes críticos com precisão [37-40].

Conforme as referências [13, 41, 50], há estreita relação entre o modelo SIR e o de percolação usual. Embora o modelo SIR tenha dinâmica temporal, enquanto o modelo de percolação é estático, pode-se analisar cada par suscetível-infectado como uma ligação através da qual pode haver transmissão da doença. Sob este ponto de vista, o processo de transmissão inicia-se a partir de um único infectado (localizado no centro da rede), que, estando interligado a primeiros vizinhos suscetíveis, pode transmitir a doença, criando novos indivíduos infectados. Após um tempo longo (no estado estacionário), a dinâmica temporal cessa devido à recuperação de todos os indivíduos infectados. Quando isso acontece, o sistema fica preso em um estado absorvente de suscetíveis e recuperados. Os indivíduos recuperados formam um aglomerado e a probabilidade de haver espalhamento da doença é definida como a probabilidade de haver percolação, ou seja, para sistemas finitos, é a probabilidade de que o aglomerado de recuperados toque pelo menos uma das fronteiras da rede.

A distribuição dos aglomerados de indivíduos recuperados, obtida em uma simulação computacional do modelo SIR, apresenta no ponto crítico propriedades que têm analogia com as do modelo de percolação [44,46]. Neste trabalho explorou-se a relação entre estes modelos e procurou-se descrever o comportamento do modelo SIR (definido em redes regulares) a partir da teoria de percolação. Para isso, primeiro foi realizada uma revisão do modelo de percolação, que será descrito no capítulo 2, no qual foram calculadas algumas grandezas importantes e discutido o seu comportamento crítico. O leitor que compreende o comportamento crítico do modelo de percolação pode iniciar a leitura a partir do capítulo 3, no qual se apresenta a investigação do modelo SIR espacialmente estruturado por meio de aproximações de campo médio dinâmico e simulações de Monte Carlo do modelo.

Para tratar explicitamente o processo de transmissão vetorial, introduziram-se dois diferentes modelos estocásticos de infecção cruzada, nos quais a transmissão da doença ocorre entre duas populações espacialmente estruturadas. Uma das populações representa os vetores e a outra representa os hospedeiros. Os indivíduos destas populações interagem entre si podendo transmitir a doença entre indivíduos de uma população para indivíduos da outra. O primeiro modelo de infecção cruzada, denominado modelo I, é descrito no capítulo 4 e visa investigar o espalhamento de uma epidemia em uma estrutura de rede regular com vetores uniformemente distribuídos. Neste modelo não foram tratados explicitamente os processos de nascimento e morte dos vetores. No segundo modelo (denominado modelo II), que será apresentado no capítulo 5, foram inseridas no modelo I reações que buscam descrever explicitamente o nascimento e morte de vetores. Ambos os 
modelos possuem dinâmicas equivalentes às regras dinâmicas do modelo SIR espacialmente estruturado, exceto que neste caso o processo de transmissão é cruzado, tornando-se dependente da existência de vetores infectados vizinhos ao hospedeiro suscetível. No modelo I, as regras de transição local são similares às regras do modelo de contato [32, 33, ,51,53] e, no modelo II, as regras análogas às do modelo SIRS [34,54,55], introduzindo uma reação reversa que descreve o processo de morte natural de um vetor.

No capítulo 6] são comparados os modelos I e II e discutidos como esses modelos podem ser usados para descrever a trasmissão da dengue em área urbana, caso haja somente um sorotipo do vírus. E, por fim, no capítulo 7 são apresentadas algumas conclusões. 


\section{Capítulo 2}

\section{Modelo de percolação}

\subsection{Introdução}

Imagine um meio com porosidade irregular, através do qual se deseja fazer passar um fluido, como se fosse um filtro natural. Para que haja escoamento do fluido, é necessário que exista pelo menos um caminho contínuo entre as extremidades. Quando isso ocorre, diz-se que há percolação e que o fluido percola o meio.

O entendimento do modelo de percolação está diretamente associado a diversos problemas, como: formação de lençóis freáticos e prospecção de petróleo [56 58], fluidez em materiais irregulares [59 61], propagação de incêndio em florestas [62], propagação de epidemias [35, 43], processos de invasão [63], condutividade elétrica em circuitos isolantes-condutores [59,63, 68 ] e difusão em misturas multicomponentes [59, 60, 64, 69].

O estudo do processo de percolação teve origem com Flory (1941) e Stockmayer (1943), que buscavam modelar o processo de formação de macromoléculas por meio da ligação de moléculas menores. A versão mais conhecida e utilizada do modelo de percolação foi introduzida em 1957 por Broadbent e Hammersley, que buscavam descrever a fluidez de partículas por um meio com cavidades distribuídas aleatoriamente.

Considerando uma rede regular, pode-se inferir que dois sítios da rede estão conectados com probabilidade $p$ ou desconectados com probabilidade $q=1-p$. Com este critério, forma-se uma estrutura com densidade de ligações ou sítios ocupados $p$ e desconexão ou sítios vazios $1-p$, e deve-se responder à seguinte questão: há sítios conectados formando um caminho contínuo entre as extremidades da superfície da rede?

Na figura 2.1, são demonstradas conexões de uma rede quadrada com $32 \times 32$ sítios, para diferentes valores da probabilidades de ligação $p$. Pode- 

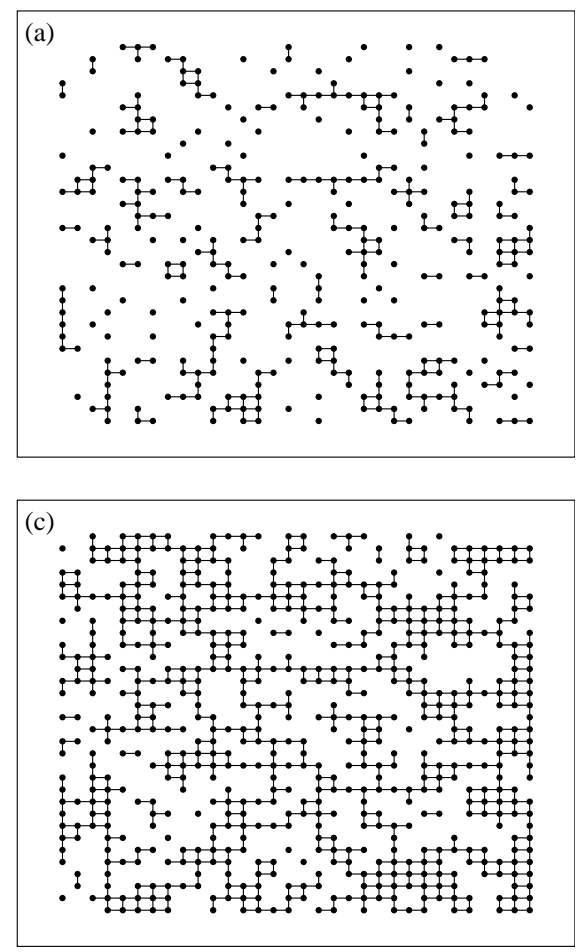
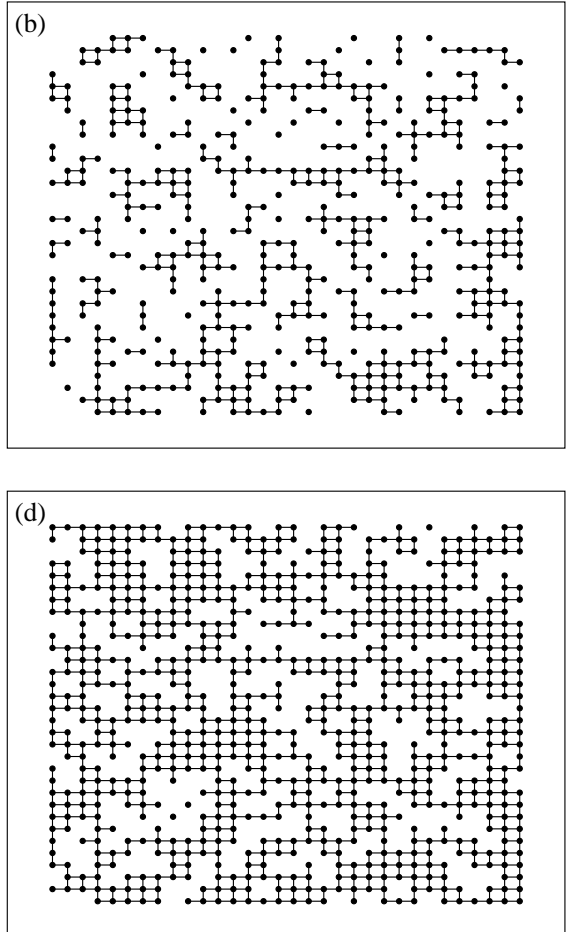

Figura 2.1: Redes de tamanho $L=32$ para diferentes probabilidades de ocupação $p$. Em (a) $p=0,35$, (b) $p=0,50$, (c) $p=0,65$ e em (d) $p=0,75$. Sítios vazios e ausência de ligações estão em branco.

se fazer a diluição da rede de duas formas distintas: remoção de sítios ou remoção de conexões. O primeiro processo corresponde ao modelo denominado percolação por sítios e o segundo, percolação por ligações. Na figura 2.1 pode-se imaginar cada sítio com um contato elétrico (ponto de solda) e cada ligação como uma resistência elétrica (todas as resistências são equivalentes). Na percolação por sítio, um contato é representado por um sítio ocupado e a ausência de contato é representada por um sítio vazio. Por sua vez, na percolação por ligação, uma conexão/desconexão representa a existência/ausência de uma resistência elétrica entre dois pontos adjacentes. Em ambos os modelos, se a probabilidade $p$ for grande, é altamente provável que, aplicando uma diferença de potencial entre duas extremidades opostas, haverá passagem de corrente elétrica (fase condutora) e, se o valor de $p$ for pequeno, é pouco provável que haja corrente elétrica (fase isolante). Se há corrente elétrica, então existe um aglomerado de resistências conectando duas extremidades adjacentes da placa de circuitos e dize-se que há percolação. A transição entre a fase isolante e a condutora está associada à probabilidade $p$ (de haver contatos no caso da percolação por sítio, ou haver resistências no caso da 
percolação por ligação). Essa transição de fase ocorre para certo limiar $p_{c}$. Abaixo do ponto crítico $\left(p<p_{c}\right)$, não há percolação e todos os aglomerados (de sítios, no caso do modelo de percolação por sítios, ou ligações, quando se tratar da percolação por ligação) têm tamanho finito. Acima do ponto crítico $\left(p>p_{c}\right)$, além de aglomerados de tamanho finito, pode haver aglomerados cujo tamanho preenche toda a extensão da rede e, no caso de uma rede infinita, haverá um aglomerado de tamanho infinito.

As propriedades críticas do modelo de percolação têm sido amplamente estudadas e apontam que o modelo e vários outros apresentam um comportamento universal no ponto crítico, pertencendo à classe de universalidade denominada classe de universal da percolação isotrópica.

Nas próximas seções serão apresentados os cálculos que descrevem probabilisticamente o processo de percolação, possibilitando determinar grandezas importantes, como o tamanho médio dos aglomerados, o limiar de percolação e o comportamento crítico do sistema para diferentes redes regulares. O formalismo apresentado neste capítulo está baseado principalmente nas referências [32, 70, 71.

Nos capítulos 3, 4 e 5, esse formalismo será usado para investigar um modelo de espalhamento de epidemia que pode ser visto como um modelo de crescimento de aglomerados cujas propriedades críticas podem ser caracterizadas a partir do conhecimento da teoria de percolação.

\subsection{Solução exata em uma dimensão}

Imagine um reticulado regular formando uma cadeia linear finita, onde cada sítio encontra-se a uma distância fixa dos seus vizinhos. Independentemente dos outros, cada sítio pode estar ocupado com probabilidade $p$ ou vazio com probabilidade $q=1-p$. Os sítios ocupados representam poros, enquanto os vazios indicam a ausência de poros.

A probabilidade de ocupação, escolhida a priori, independe dos estados dos sítios vizinhos. Para descrever uma configuração do sistema, associa-se a cada sítio $i$ uma variável estocástica $\eta_{i}$ que toma os valores $\eta_{i}=1 \mathrm{ou}$ $\eta_{i}=0$, conforme o sítio esteja ocupado ou vazio, respectivamente. Assim uma configuração é representada pelo vetor $\eta=\left(\eta_{1}, \eta_{2}, \eta_{3}, \ldots, \eta_{N-1}, \eta_{N}\right)$, em que $N$ é o total de sítios do reticulado.

Um aglomerado ou "cluster" é um conjunto de sítios ocupados e conectados. Como os sítios são ocupados independentemente com probabilidade $p$, a probabilidade de dois sítios quaisquer estarem ocupados é $p^{2}$. Para um tripleto, isto é, um trio de três sítios ocupados, esta probabilidade será $p^{3}$ e assim sucessivamente. Por outro lado, a probabilidade de se ter um sítio 
vazio é $q=1-p$ e a probabilidade de haver dois sítios quaisquer vazios é $q^{2}=(1-p)^{2}$. Para haver um aglomerado de $s$ sítios, é necessário que os $s$ sítios estejam ocupados, formando uma cadeia contínua, sem sítios vazios entre eles, e nos extremos direito e esquerdo, deve haver sítios vazios delimitando o aglomerado. Por exemplo, a probabilidade de se ter um aglomerado de dois sítios é $q p^{2} q=p^{2}(1-p)^{2}$.

Além de saber o tamanho dos aglomerados, é possível calcular o número de aglomerados de tamanho $s$ existentes na rede. Como cada sítio tem probabilidade $p^{s}(1-p)^{2}$ de pertencer ao aglomerado, para uma rede de tamanho $L$, desprezando efeitos de borda, o total de aglomerados por sítio $s$ é $L p^{s}(1-p)^{2}$. Entretanto, é conveniente calcular grandezas que independem do tamanho da rede. Por isso, define-se $n_{s}$ como o número médio de aglomerados de tamanho $s$ por sítio. No caso unidimensional, tem-se:

$$
n_{s}=p^{s}(1-p)^{2} .
$$

A probabilidade de que um sítio, escolhido aleatoriamente, faça parte de um aglomerado de tamanho $s$ é $s n_{s}$, uma vez que ele pode ser qualquer um $\operatorname{dos} s$ sítios do aglomerado. Quando a probabilidade de ocupação é $p=1$, toda a rede está ocupada, formando um único aglomerado. Contudo, se $p<1$, haverá sítios vazios, totalizando $(1-p) L$ "buracos" na rede, em que $L$ é o tamanho da rede. Portanto, não haverá linha contínua conectando os extremos da rede. Consequentemente, o limiar de percolação no caso unidimensional é a unidade

$$
p_{c}=1 \text {. }
$$

Todo sítio ocupado pertence a um aglomerado, mesmo que ele esteja isolado, formando um aglomerado de tamanho unitário. Assim, a probabilidade de um sítio qualquer pertencer a um aglomerado é exatamente a probabilidade $p$ de ele estar ocupado. Isto é, ao somar sobre todos os aglomerados de tamanho $s$, deve-se obter a probabilidade de que o sítio pertença a um deles

$$
\sum_{s=1}^{\infty} n_{s} s=p .
$$

Esse resultado pode ser demonstrado como a seguir:

$$
\begin{aligned}
& \sum_{s=1}^{\infty} n_{s} s=\sum_{s=1}^{\infty} p^{s}(1-p)^{2} s=(1-p)^{2} \sum_{s=1}^{\infty} s p^{s} \\
& =(1-p)^{2} \sum_{s=1}^{\infty} p \frac{d}{d p} p^{s}=p(1-p)^{2} \frac{d}{d p} \sum_{s=1}^{\infty} p^{s}
\end{aligned}
$$


Resolvendo a série geométrica, lembrando que ela só é convergente se $p<p_{c}=1$, obtem-se:

$$
\sum_{s=1}^{\infty} p^{s}=p+p^{2}+p^{3}+p^{4}+\ldots=\frac{p}{1-p} .
$$

Logo, substituindo o resultado obtido na equação (2.5) na equação (2.4), obtem-se:

$$
\sum_{s=1}^{\infty} n_{s} s=\sum_{s=1}^{\infty} p^{s}(1-p)^{2} s=p(1-p)^{2} \frac{d}{d p}\left(\frac{p}{1-p}\right)=p .
$$

Ao realizar a soma sobre todos os tamanhos de aglomerados, deve-se excluir aglomerados de tamanho infinito. No caso unidimensional ele não existe enquanto $p<1$.

Assim, $\sum_{s} n_{s} s$ é a probabilidade de um sítio pertencer a um aglomerado. Desta definição, entende-se que a probabilidade de um sítio escolhido aleatoriamente pertencer a um aglomerado de tamanho $s$ é:

$$
w_{s}=\frac{n_{s} s}{\sum_{s=1}^{\infty} n_{s} s} \text {. }
$$

O tamanho médio $S$ de um aglomerado é dado por

$$
S=\sum_{s=1}^{\infty} s w_{s}=\sum_{s=1}^{\infty} \frac{n_{s} s^{2}}{\sum_{s=1}^{\infty} n_{s} s} .
$$

A soma que aparece no denominador já foi calculada na equação (2.6) e vale $p$. Para resolver a soma do numerador, utiliza-se o mesmo métdo de obter a série geométrica e depois derivar o resultado. O cálculo a ser feito é:

$$
\begin{array}{r}
\sum_{s=1}^{\infty} n_{s} s^{2}=\sum_{s=1}^{\infty} p^{s}(1-p)^{2} s^{2}=(1-p)^{2} \sum_{s=1}^{\infty} s^{2} p^{s} \\
=(1-p)^{2} p \frac{d}{d p} \sum_{s=1}^{\infty} s p^{s}=(1-p)^{2} p \frac{d}{d p}\left[p \frac{d}{d p}\left(\sum_{s=1}^{\infty} p^{s}\right)\right] \\
=p(1-p)^{2} \frac{d}{d p}\left[p \frac{d}{d p}\left(\frac{p}{1-p}\right)\right]=p(1-p)^{2} p \frac{d}{d p}\left[p \frac{d}{d p}\left(\frac{p}{1-p}\right)\right] \\
=\frac{p}{1-p}(1-p+2 p)=p \frac{1+p}{1-p}, \quad\left(p<p_{c}\right) .
\end{array}
$$

E, portanto,

$$
S=\frac{1+p}{1-p}, \quad\left(p<p_{c}\right)
$$


Percebe-se que o tamanho médio do aglomerado diverge conforme se aproxima do ponto crítico.

Define-se a função de correlação ou conectividade de pares $g(r)$ como a probabilidade de que dois sítios a uma distância $r$ pertençam ao mesmo aglomerado. Se $r=0$, então $g(0)=1$. Para $r=1$, os sítios pertencem ao mesmo aglomerado se, e somente se, eles são vizinhos e estão ocupados. Para sítios distando $r$ um do outro, para que estejam no mesmo aglomerado, é necessário que os $r-1$ sítios entre eles estejam ocupados. Isso ocorre com probabilidade $p^{r}$ e, assim

$$
g(r)=p^{r}, \quad(\forall \quad p \quad \text { e } \quad r) .
$$

Para $p<p_{c}$, a correlação vai a zero exponencialmente se a distância vai a infinito, ou seja,

$$
g(r)=e^{-\frac{r}{\xi}}
$$

em que

$$
\xi=-\frac{1}{\ln p}=-\frac{1}{\ln \left(1+p-p_{c}\right)} \approx \frac{1}{p_{c}-p}, \quad\left(p \rightarrow p_{c}\right) .
$$

A grandeza $\xi$ é o comprimento de correlação ou conectividade.

Tanto $\xi$ quanto $S$ dão uma medida do tamanho médio dos aglomerados, entretanto, há uma distinção crucial entre eles. O comprimento de correlação $\xi$ deve ser entendido como o comprimento linear dos aglomerados, enquanto a grandeza $S$ deve ser entendida como o número de sítios ocupados no aglomerado. Essas duas grandezas estão relacionadas por meio de

$$
S=\sum_{r=0}^{\infty} g(r) .
$$

\subsection{Extensão para redes d-dimensionais}

Ao analisar o problema de percolação, qual deve ser a probabilidade $P_{s}$ de que determinado sítio pertença a um aglomerado de tamanho $s$ ? Para responder a essa pergunta, é mais conveniente indagar primeiro qual é a probabilidade de haver um aglomerado de $s$ sítios, que pode também ser entendida como o número médio (por sítio) $n_{s}$ de aglomerados contendo $s$ sítios. A relação entre $P_{s}$ e $n_{s}$ é

$$
P_{s}=s n_{s}
$$

Como exemplo, pode-se usar uma rede quadrada. Nessa rede, usando $s=1$ na equação (2.15) , tem-se a probabilidade de haver um aglomerado com 
um único sítio. Para isso ocorrer, é necessário que ele esteja ocupado com probabilidade $p$ e seus quatro vizinhos devem estar vazios (com probabilidade $1-p)$. Assim, a probabilidade de se obterem aglomerados com somente um sítio é $n_{1}=p(1-p)^{4}$.

Ao calcular a probabilidade de encontrar aglomerados com dois sítios $(s=2)$, o par de sítios ocupados pode estar tanto na horizontal quanto na vertical e, então, haver seis vizinhos que precisam estar vazios. Deste modo, $n_{2}=2 p^{2}(1-p)^{6}$. No caso de aglomerados de três sítios, há dois tipos de aglomerados: serão considerados primeiramente apenas os aglomerados lineares, para um aglomerado de três sítios que possui oito sítios na periferia, de modo que $n_{3}=2 p^{3}(1-p)^{8}$.

Generalizando para aglomerados de $s$ sítios colineares na rede quadrada, obtem-se

$$
n_{s}=2 p^{s}(1-p)^{2 s+2} .
$$

Nesta equação, o fator 2 advém do número de configurações distintas e está relacionado à liberdade de se fazer o alinhamento horizontal ou vertical.

Em uma rede cúbica regular, cada sítio tem seis vizinhos e, portanto, cada aglomerado linear tem $2(2 s+1)$ sítios vizinhos e tem-se 3 graus de liberdade para orientar a linha. Portanto, a probabilidade de se ter um aglomerado com $s$ sítios é

$$
n_{s}=3 p^{s}(1-p)^{2+4 s} \text {. }
$$

Para um rede hipercúbica d-dimensional, cada sítio tem $2 d$ vizinhos e, consequentemente, um aglomerado linear com $s$ sítios terá $(2 d-2) s$ vizinhos vazios e mais os 2 vizinhos que delimitam as extremidades. Portanto, a probabilidade de haver um aglomerado de $s$ sítios deve ser

$$
n_{s}=d p^{s}(1-p)^{2+(2 d-2) s}
$$

Entretanto, até o momento só foram considerados aglomerados lineares e não há motivo para os aglomerados serem todos assim. Para formar um trio em uma rede quadrada, há sete vizinhos vazios delimitando o aglomerado e, combinando este resultado ao obtido para o caso linear, a probabilidade de existência de aglomerados de tamanho $s=3$ para a rede quadrada é dada por

$$
n_{3}=2 p^{3}(1-p)^{8}+4 p^{3}(1-p)^{7} .
$$

Com o aumento do tamanho do aglomerado, obter as diferentes configurações vai se tornando dispendioso, sendo conveniente fazer este cálculo computacionalmente. Ao calcular $n_{s}$, é necessário ter o número de sítios vazios que delimitam o aglomerado. Essa grandeza é denominada perímetro e não deve ser confundida com a superfície, pois o aglomerado não 
necessariamente será compacto, podendo apresentar buracos. Usa-se o símbolo $t$ para denotar o perímetro, e o número de configurações de aglomerados com $s$ sítios é $g_{s t}$. A partir destas definições, a expressão geral para $n_{s}$ tornase

$$
n_{s}=\sum_{t} g_{s t} p^{s}(1-p)^{t}
$$

\subsection{Solução exata na árvore de Cayley}

A árvore de Cayley é construída da seguinte forma: partindo de um sítio, conectam-se a ele $z$ sítios. Isso define a primeira camada concêntrica ou geração. Por regra de construção, cada sítio deve fazer $z$ conexões. Então, na segunda geração, cada sítio deve fazer $\sigma=z-1$ novas conexões. com sítios da terceira geração, e assim sucessivamente. Na figura 2.2, consta um exemplo de árvore com coordenação $z=3$. Desta forma, partindo de um sítio, a cada ramo ou conexão, encontram-se $\sigma$ novas ramificações levando a novos vizinhos, que podem estar ocupados cada um com probabilidade $p$. Logo, tem-se em média $\sigma p$ novos vizinhos para estabelecer um caminho contínuo. Se $\sigma p<1$, o número de conexões para formar um caminho infinito decresce a cada ramo por um fator menor que a unidade e a probabilidade de encontrar um caminho infinito vai a zero exponencialmente. Portanto,

$$
p_{c}=\frac{1}{z-1}
$$

é o limiar de percolação para a árvore de Cayley com coordenação z.

$\mathrm{Na}$ árvore de Cayley, qualquer aglomerado de $s$ sítios possui o mesmo número de sítios na periferia. Essa propriedade importante diz que $t$ é univocamente determinado por $s$ e é dada por $t=(\sigma-1) s+2$. Portanto, para uma árvore de Cayley, o número médio de aglomerados de tamanho $s$ por sítio se escreve como

$$
n_{s}=b_{s} p^{s}(1-p)^{(\sigma-1) s+2}
$$

em que $b_{s}$ é um coeficiente que depende apenas do tamanho $s$ do aglomerado.

O número médio de aglomerados por sítio é dado pela expressão

$$
f=\sum_{s=1}^{\infty} n_{s}=\sum_{s=1}^{\infty} b_{s} p^{s}(1-p)^{(\sigma-1) s+2}=q^{2} \sum_{s=1}^{\infty} b_{s} p^{s} q^{(\sigma-1) s} .
$$

O número médio de sítios pertencentes a aglomerados finitos, por sítio, é

$$
F=\sum_{s=1}^{\infty} s n_{s}=q^{2} \sum_{s=1}^{\infty} s b_{s} p^{s} q^{(\sigma-1) s}
$$




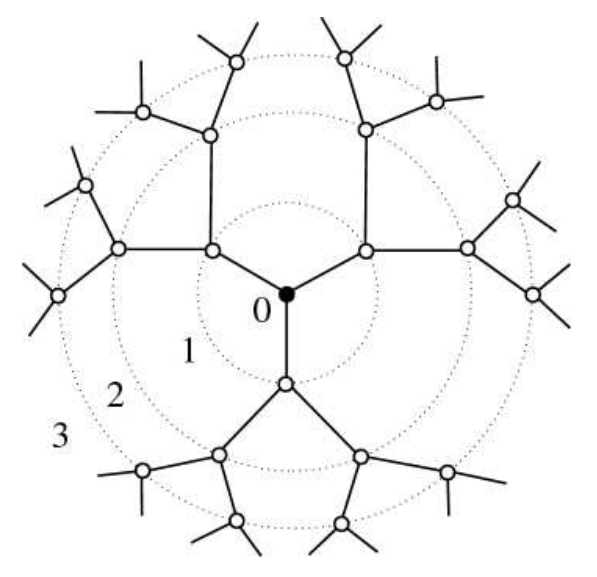

Figura 2.2: Exemplo de árvore de Cayley com coordenação $z=3$. Na imagem estão enumeradas as gerações. O número de sítios em cada geração cresce como uma lei de potência em função do aumento da geração.

E o tamanho médio dos aglomerados finitos é

$$
S=\sum_{s=1}^{\infty} s^{2} n_{s}=q^{2} \sum_{s=1}^{\infty} s^{2} b_{s} p^{s} q^{(\sigma-1) s} .
$$

As expressões (2.23), (2.24) e (2.25) dependem dos coeficientes $b_{s}$, que podem ser obtidos usando a equação (2.24), pois $F=p$ para $p<p_{c}$, caso em que a probabilidade de percolação $P$ se anula.

Para se obterem as grandezas $f, F$ e $S$ sem calcular os coeficientes $b_{s}$, utiliza-se o método da função geratriz. Define-se a função geratriz $G(x)$ por

$$
G(x)=\sum_{s=1}^{\infty} b_{s} x^{s}
$$

em que $x=p q^{\sigma-1}$. A partir da função geratriz, tem-se que

$$
\begin{array}{r}
f=q^{2} G(x) \\
F=q^{2} x \frac{d G}{d x} \\
S=q^{2}\left(x^{2} \frac{d^{2} G}{d x^{2}}+x \frac{d G}{d x}\right) .
\end{array}
$$

Como abaixo do ponto crítico a probabilidade de um sítio pertencer a qualquer cluster finito é $p$, então $F=p$, tornand-se possível escrever

$$
F=q^{2} x G^{\prime}(x)=p, \quad p<p_{c} .
$$


Usando a definição de $x$, obtem-se a primeira derivada de $G$ com relação a $x$

$$
G^{\prime}(x)=q^{-(\sigma+1)} .
$$

Lembrando-se que $q$ depende implicitamente de $x$.

Para obter a derivada segunda de $G$ em relação a $x$, aplica-se a regra da cadeia:

$$
\frac{d^{2} G}{d x^{2}}=\frac{d G^{\prime}}{d x}=\frac{d q}{d x} \frac{d G^{\prime}}{d q} .
$$

Por outro lado,

$$
\frac{d x}{d q}=\frac{d}{d q}\left((1-q) q^{\sigma-1}\right)=q^{\sigma-2}(\sigma-1-\sigma q)
$$

$\mathrm{e}$

$$
\frac{d G^{\prime}}{d q}=-(\sigma+1) q^{-\sigma-2} .
$$

Substituindo as equações (2.33) e (2.34) na equação (2.32), obtem-se

$$
G^{\prime \prime}(x)=\frac{1}{q^{\sigma-2}(\sigma-1-\sigma q)}\left[-(\sigma+1) q^{-\sigma-2}\right]=(\sigma+1) \frac{q^{-2 \sigma}}{1-\sigma p} .
$$

Substituindo $G^{\prime}(x)$ e $G^{\prime \prime}(x)$ na equação (2.29) determina-se o tamanho médio dos aglomerados

$$
\begin{aligned}
S & =q^{2}\left[x^{2} \frac{d^{2} G}{d x^{2}}+x \frac{d G}{d x}\right]=q^{2} p q^{\sigma-1}\left[p q^{\sigma-1} \frac{(\sigma+1) q^{-2 \sigma}}{1-\sigma p}+q^{-\sigma-1}\right] \\
& =p q^{\sigma+1-\sigma-1}\left[p \frac{1+\sigma}{1-\sigma p}+1\right]=p \frac{1+p}{1-\sigma p} ; \quad\left(p<p_{c}\right) .
\end{aligned}
$$

Note que $S$ diverge quando $p=1 / \sigma$. Conforme a equação (2.21), a probabilidade crítica é

$$
p_{c}=\frac{1}{\sigma}=\frac{1}{z-1} .
$$

A equação (2.37) pode ser reescrita como

$$
S=\frac{p(1+p)}{\sigma(1 / \sigma-p)}=\frac{p(1+p)}{\sigma\left(p_{c}-p\right)}
$$

A partir desta expressão, é possível notar que o comportamento crítico da função $S$ é dado por

$$
S \sim\left(p_{c}-p\right)^{-1}
$$


Para calcular o número médio de aglomerados por sítio, é necessário expressar $G(x)$ explicitamente. Para isso é suficiente integrar a função $G^{\prime}(x)$ e determinar a constante de integração.

$$
\begin{aligned}
G(x) & =\int q^{-(\sigma+1)} \frac{d x}{d q} d q=\int q^{-(\sigma+1)} q^{\sigma-2}(\sigma-1-\sigma q) d q \\
& =c-\frac{(\sigma-1) q^{-2}}{2}+\sigma q^{-1}
\end{aligned}
$$

Note que, por definição, a equação (2.26) tem a propriedade $G(0)=0$. Por outro lado, quando $x=0$, temos $p q^{\sigma-1}=0$ e portanto $q=1$. Com este resultado, é possível determinar a constante de integração, $c=-(\sigma+1) / 2$. E a função geratriz, abaixo do limiar de percolação, é

$$
G(x)=-\frac{1}{2}\left(\frac{\sigma-1}{q^{2}}-2 \frac{\sigma}{q}+(\sigma+1)\right) .
$$

E com a substituição da equação (2.42) na equação (2.27), pode-se escrever o número de aglomerados por sítio como $f=-\frac{1}{2}\left[(\sigma-1)-2 \sigma q+(\sigma+1) q^{2}\right]$. Substituindo $q=1-p$, obtem-se

$$
f=p-\frac{\sigma+1}{2} p^{2}
$$

\subsection{Comportamento crítico na árvore de Cay- ley}

No limite em que $p \rightarrow p_{c}$, o tamanho médio dos aglomerados diverge conforme a expressão

$$
S \sim\left(p_{c}-p\right)^{-1}
$$

e a probabilidade de percolação

$$
P \sim\left(p-p_{c}\right), \quad\left(p>p_{c}\right) .
$$

Para efeito de comparação, pode-se calcular a razão entre o número de aglomerados de tamanho $s$ quando a probabilidade de ocupação é $p$ e quando é $p=p_{c}$.

No limite $p \rightarrow p_{c}$, para aglomerados de tamanho grande

$$
n_{s}(p) \propto e^{-c s},
$$

em que a constante $c$ é dada pela expressão

$$
c \sim\left(p-p_{c}\right)^{2} .
$$


Mesmo sem calcular o número de configurações $b_{s}$, pode-se obter o comportamento assintótico do número médio de aglomerados no limiar, $n_{s}\left(p_{c}\right)$. Pela equação (2.8), tem-se

$$
S \propto \sum_{s=1}^{\infty} s^{2} n_{s},
$$

pois o denominador na equação (2.8) é finito no limiar. Esta soma também é denominada segundo momento da distribuição dos tamanhos dos aglomerados. Se $n_{s}\left(p_{c}\right)$ decai exponencialmente com $s$, então $S$ deve ser finito em $p_{c}$. Desta forma, $S$ deve comportar-se como uma lei de potência

$$
n_{s}\left(p_{c}\right) \sim s^{-\tau}
$$

em que $\tau$ é o expoente de Fisher. Ao calcular $S$, estar-se determinando $\tau$, pois depois pode-se simplesmente comparar o resultado com a equação (2.44).

Para $p$ menor que $p_{c}$, mas muito próximo, tem-se:

$$
\begin{aligned}
& S \propto \sum_{s=1}^{\infty} s^{2} n_{s} \propto \sum_{s=1}^{\infty} s^{2-\tau} e^{-c s} \propto \int_{0}^{\infty} s^{2-\tau} e^{-c s} d s \\
= & c^{\tau-3} \int_{0}^{\infty} x^{2-\tau} e^{-x} d x \propto c^{-\tau+3} \propto\left(p_{c}-p\right)^{-2(\tau-3)} .
\end{aligned}
$$

A equação (2.44) mostra que $S$ diverge com expoente -1 e, portanto,

$$
2(\tau-3)=1
$$

Logo, o expoente de Fisher é

$$
\tau=5 / 2
$$

na árvore de Cayley.

Substituindo o valor de $\tau$ na equação (2.46), obtem-se:

$$
n_{s}(p) \sim s^{-5 / 2} e^{-c s}, \quad\left(c \propto\left(p-p_{c}\right)^{2}\right) .
$$

A expressão para $n_{s}(p)$ é válida para todo $p \operatorname{com} s$ grande. Já o valor para a constante $c$ exige que $p \rightarrow p_{c}$.

\subsection{Comportamento crítico}

Na seção (2.2), foram obtidos resultados exatos para o caso unidimensional e, na seção (2.4), foram encontrados resultados para a árvore de Cayley. Então, é possível estipular o comportamento das grandezas calculadas para redes d-dimensionais. 
As equações (2.1) e (2.53), referentes ao caso unidimensional e na árvore de Cayley, são dominadas por aglomerados grandes, seguindo um decaimento exponencial. Em uma rede regular, o decaimento pode não ser necessariamente exponencial, de modo que postulamos o resultado geral

$$
n_{s}=s^{-\tau} f\left(\left(p-p_{c}\right) s^{\sigma}\right) \text {. }
$$

em que $\sigma$ é um expoente crítico, o qual, para a árvore de Cayley, é $1 / 2$ e 1 no caso unidimensional.

Se um sítio está ocupado com probabilidade $p$, então ele pertence a um aglomerado de tamanho infinito ou a um de tamanho finito. Isto é,

$$
p=P+\sum_{s=1}^{\infty} s n_{s}
$$

No ponto crítico, tem-se $p=p_{c}$ e $P=0$ e, assim, $p_{c}=\sum_{s=1}^{\infty} s n_{s}$. Para essa soma convergir, é necessário que $\tau>2$. Deste modo, é possível reescrever a equação (2.55) como

$$
\begin{aligned}
& P=\sum_{s=1}^{\infty}\left[n_{s}(p)-n_{s}\left(p_{c}\right)\right] s \\
& \propto \sum_{s=1}^{\infty} s^{1-\tau}[f(x)-f(0)],
\end{aligned}
$$

em que $x=\left(p-p_{c}\right) s^{\sigma}$. Se $p$ estiver próximo de $p_{c}$, o fator $c$ no expoente da equação (2.50) deve ser muito pequeno, e somente valores de $s$ grande, da ordem de $1 / c$, devem ser significantes na soma. Neste limite de $p \rightarrow$ $p_{c}$ e $s \rightarrow \infty$, é possível substituir a soma por uma integral, visto que há interesse somente no comportamento na criticalidade. Portanto, satisfeitas estas condições, tem-se:

$$
P \propto \int_{0}^{\infty} s^{1-\tau}[f(x)-f(0)] d s .
$$

Ao usar ntegração por partes, substituindo $d x=(\sigma x / s) d s$,

$$
P \propto\left|p-p_{c}\right|^{(2-\tau) / \sigma} \int_{0}^{\infty} x^{-1+(2-\tau) / \sigma}[f(x)-f(0)] d x / \sigma .
$$

O termo constante resultante da integração se anula, desde que $\tau<3$. Ocorrendo isto,

$$
P \propto c^{2-\tau} \propto\left(p-p_{c}\right)^{(\tau-2) / \sigma}=\left(p-p_{c}\right)^{\beta}
$$


em que o expoente crítico

$$
\beta=\frac{\tau-2}{\sigma}
$$

Calculando também o tamanho médio dos aglomerados $S$, que diverge no ponto crítico, conforme a equação (2.50), tem-se:

$$
S \propto \sum_{s=1}^{\infty} s^{2} n_{s},
$$

que no ponto crítico é

$$
S=\frac{\sum_{s=1}^{\infty} s^{2} n_{s}}{p_{c}}
$$

Como discutido anteriormente, em torno do ponto crítico pode-se trocar a soma por uma integral. Assim,

$$
\begin{array}{r}
S \propto \int_{0}^{\infty} s^{2} n_{s} d s \\
\propto \int_{0}^{\infty} s^{2-\tau} f(x) d s .
\end{array}
$$

Com a mudança de variável $x=c s$ :

$$
S \propto\left|p-p_{c}\right|^{-(3-\tau) / \sigma} \int_{0}^{\infty} x^{-1+(3-\tau) / \sigma} f(x) d x / \sigma .
$$

Portanto, $S$ diverge no ponto crítico com o expoente $\gamma$,

$$
S \propto\left|p-p_{c}\right|^{-(3-\tau) / \sigma}=\left|p-p_{c}\right|^{-\gamma} .
$$

Logo,

$$
\gamma=\frac{3-\tau}{\sigma}
$$

Uma vez que $\beta$ e $\gamma$ são positivos, é necessário que $2<\tau<3$.

Ao calcular o k-ésimo momento da distribuição de tamanhos de aglomerados,

$$
M_{k}=\sum_{s=1}^{\infty} s^{k} n_{s}
$$

$\mathrm{Na}$ vizinhança do ponto crítico, vale a relação

$$
\begin{aligned}
& M_{k} \propto \sum_{s=1}^{\infty} s^{k-\tau} s^{\sigma} \\
\propto & \int_{0}^{\infty} s^{k-\tau} f(x) d s .
\end{aligned}
$$




$$
M_{k} \propto\left|p-p_{c}\right|^{(\tau-k-1) / \sigma} \int_{0}^{\infty} x^{-1-(\tau-k-1) / \sigma} f(x) d x / \sigma
$$

O expoente $(k+1-\tau) / \sigma$ pode ser expresso em termos de $\tau$ e $\sigma$ e, portanto, $\tau$ e $\sigma$ não são independentes. Pode-se escolher $\sigma$ e $\tau$ como expoentes fundamentais ou $\beta$ e $\gamma$.

Pelas equações (2.60) e (2.65), tem-se as seguintes relações:

$$
\frac{\beta}{\gamma}=\frac{\tau-2}{3-\tau} \quad \therefore \quad \tau=2+\frac{\beta}{\beta+\gamma} .
$$

Substituindo este resultado na equação (2.60), obtem-se:

$$
\sigma=\frac{1}{\beta+\gamma}
$$

Em termos de $\beta$ e $\gamma$ pode-se escrever o terceiro momento, denotando simplesmente por $M$, ocultando-se o subíndice, na forma

$$
M \sim\left|p-p_{c}\right|^{(\tau-3-1) / \sigma}=\left|p-p_{c}\right|^{(\beta+2 \gamma)} .
$$

A probabilidade de se encontrarem aglomerados de tamanho $s$ no ponto crítico é $P_{s}=s n_{s} \sim s^{1-\tau}$ e de encontrarem aglomerados de tamanho finito maior ou igual a $s$ é a soma acumulada

$$
P_{\geqslant s}=\sum_{s}^{\infty} p_{s} \sim \int_{s}^{\infty} s^{1-\tau} f(x) d s .
$$

No ponto crítico, essa probabilidade acumulada deve se comportar da forma

$$
P_{\geqslant s} \sim s^{2-\tau} .
$$

E nas proximidades do ponto crítico, deve ser uma função com o seguinte comportamento

$$
P_{\geqslant s} \sim s^{2-\tau} F\left(\epsilon s^{\sigma}\right),
$$

em que $\epsilon=p-p_{c}$ é o desvio em relação ao ponto crítico. O comportamento crítico dessas grandezas serão importantes para analisar as propriedades críticas do modelo SIR, que também aparecem na descrição dos modelos epidêmicos que serão discutidos nos capítulos 4 e 5 . 


\section{Capítulo 3}

\section{Modelo \\ Suscetível-Infectado-Recuperado (SIR)}

\subsection{Descrição do Modelo}

O modelo suscetível-infectado-recuperado (SIR) foi introduzido como um modelo fenomenológico para descrever a dinâmica de doenças infecto-contagiosas [15]. Neste modelo cada indivíduo da população pode estar em um dos seguintes estados: suscetível (S), infectado (I) ou recuperado (removido e imune) (R) e permanentemente imunizado. Considerase que a epidemia tenha uma duração muito menor que o tempo de vida do hospedeiro, hipótese que é válida para várias doenças [2, 3, 6, 17, 14, 16 - 20], e assim, durante o intervalo de tempo em que ocorre a epidemia, assume-se que a população seja constante.

Para a mecânica estatística, este modelo é importante por exibir uma transição de fase de não-equilíbrio contínua entre uma fase em que a epidemia se espalha e outra na qual, devido à taxa de infecção ser muito baixa, a doença não se espalha. Como tanto o processo de infecção quanto o processo de recuperação são irreversíveis, o sistema sempre evolui para um estado absorvente.

Na dinâmica de evolução, adota-se que toda a população esteja suscetível em relação a uma doença, e, portanto, a disseminação da doença ocorre devido a uma pequena perturbação (uma pequena densidade de infectados). O processo de infecção é catalítico $(S I \rightarrow I I)$, enquanto o de recuperação e imunização é espontâneo $(I \rightarrow R)$. Na figura 3.1, consta um diagrama das reações locais para esse modelo. Nota-se que, quando o indivíduo torna- 
se recuperado, seu estado não muda mais, e que todas as transições não apresentam reação reversa.

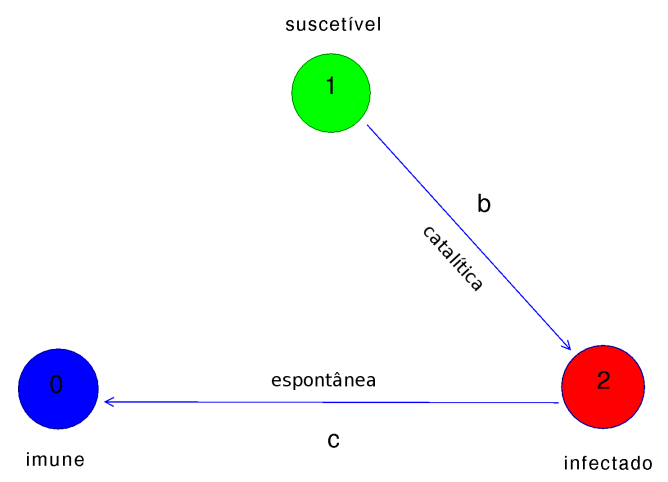

Figura 3.1: Esboço das transições entre os estados para o modelo SIR.

Neste capítulo investigamos o modelo SIR definido em uma rede regular evoluindo com dinâmica temporal assíncrona na qual apenas um sítio é atualizado a cada instante. Este é um modelo com população espacialmente estruturada [1]. Cada sítio da rede representa um indivíduo, ao qual associamos uma variável estocástica $\eta_{i}$ que assume os valores $\eta_{i}=0,1$ ou 2 , conforme o indivíduo esteja recuperado, suscetível ou infectado, respectivamente. Os processos de infecção e recuperação são tratados como reações locais em que cada indivíduo suscetível pode se tornar infectado com taxa $b$ se houver pelo menos um primeiro vizinho infectado.

Em uma rede regular em que cada sítio tenha $\zeta$ primeiros vizinhos, a probabilidade de um indivíduo suscetível se tornar infectado é $\frac{b}{\zeta} n$, em que $n$ é o número de primeiros vizinhos infectados. Uma vez infectado, o indivíduo pode se recuperar espontaneamente $\operatorname{com}$ probabilidade $c$, ficando permanentemente recuperado e imune.

O processo SIR a ser descrito aqui é um processo markoviano [72, 73] a tempo contínuo e isso permite reescalar o tempo de modo a considerar:

$$
b+c=1,
$$

em que $b$ e $c$ são probabilidades reduzidas. Adotando a probabilidade de imunização $c$ como parâmetro de controle, tem-se $b=1-c$.

Na seção 3.2 é apresentada a descrição do modelo por meio da equação mestra. Com este formalismo, obte-se as equações de evolução temporal das densidades de suscetíveis, infectados e recuperados. Se essas equações forem tratadas por meio de aproximação de campo médio simples, obtem-se equações idênticas às obtidas por Kermack e McKendrik [15]. Ainda dentro 
do contexto das aproximações de campo médio, será analisado o modelo por meio de aproximações de pares.

A caracterização do comportamento crítico do modelo é feita por meio de simulações computacionais, e será detalhada nas seções 3.5 à 3.8 .

\subsection{Equação mestra para o modelo SIR}

Considerando um reticulado com $N$ sítios e representando cada configuração do sistema pelo vetor

$$
\eta=\left(\eta_{1}, \eta_{2}, \ldots, \eta_{i}, \ldots, \eta_{N}\right),
$$

em que $\eta_{i}$ é uma variável estocástica associada ao i-ésimo sítio e que pode assumir um dos seguintes valores: $\eta_{i}=1$ (sítio ocupado por um único indivíduo suscetível), $\eta_{i}=2$ (sítio ocupado por um único indivíduo infectado) ou $\eta_{i}=0$ (sítio ocupado por um único indivíduo recuperado). A probabilidade $P(\eta, t)$ de o sistema estar em uma configuração microscópica $\eta$ no instante $t$ obedece à equação mestra [12, 31, 32, 39],

$$
\frac{d}{d t} P(\eta, t)=\sum_{\eta^{\prime} \neq \eta}\left\{W\left(\eta \mid \eta^{\prime}\right) P\left(\eta^{\prime}, t\right)-W\left(\eta^{\prime} \mid \eta\right) P(\eta, t)\right\}
$$

em que a soma é feita sobre todas as configurações $\eta^{\prime}$ (diferente de $\eta$ ); $W\left(\eta \mid \eta^{\prime}\right) \geqslant 0$ para $\eta^{\prime}$ diferente de $\eta$, denota a taxa de transição de $\eta^{\prime}$ para $\eta$.

Para calcular grandezas de estado como, por exemplo, a densidade de indivíduos recuperados, utiliza-se a equação mestra (3.3). Antes de prosseguir aos cálculos deve-se estabelecer a dinâmica de evolução temporal e construir a equação mestra para o modelo SIR estocástico e espacialmente estruturado, que é o objeto de estudo. Nesse trabalho, considerou-se uma dinâmica temporal assíncrona, na qual apenas um sítio é atualizado por unidade de tempo. Nesse caso, a taxa de transição do microestado $\eta^{\prime}$ para $\eta$ é escrita como

$$
W\left(\eta \mid \eta^{\prime}\right)=\sum_{i=1}^{N} \delta\left(\eta_{1}, \eta_{1}^{\prime}\right) \delta\left(\eta_{2}, \eta_{2}^{\prime}\right) \ldots \delta\left(\eta_{i}^{i}, \eta_{i}^{\prime}\right) \ldots \delta\left(\eta_{N}, \eta_{N}^{\prime}\right) \omega_{i}\left(\eta^{\prime}\right)
$$

em que $\delta(\alpha, \beta)$ é o delta de Kronecker e $\omega_{i}\left(\eta^{\prime}\right)$ denota a probabilidade de transição do i-ésimo sítio da configuração $\eta^{\prime}$; e $\eta^{i}$ (índice superescrito à direita) denota o estado obtido de $\eta^{\prime}$ pela atualização do i-ésimo sítio que muda de estado na seguinte ordem $(1 \rightarrow 2,2 \rightarrow 0,0 \rightarrow 1))$. 
A média da grandeza de estado $f(\eta)$ é calculada a partir da distribuição de probabilidade $P(\eta, t)$ e é definida por

$$
<f(\eta)>=\sum_{\eta} f(\eta) P(\eta, t) .
$$

Utilizando as equações (3.3), (3.4) e (3.5), obtem-se a equação de evolução temporal para $<f(\eta)>$,

$$
\begin{aligned}
\frac{d}{d t}<f(\eta)>= & \sum_{i}^{N} \sum_{\eta} \sum_{\eta^{\prime}}\left\{f\left(\eta_{1}^{\prime}, \ldots, \eta_{i}^{\prime}, \ldots, \eta_{N}^{\prime}, t\right) \delta\left(\eta_{i}, 1-\eta_{i}^{\prime}\right)\right. \\
& \left.\prod_{\substack{j=1 \\
(j \neq i)}}^{N} \delta\left(\eta_{j}, \eta_{j}^{\prime}\right) w_{i}\left(\eta_{1}^{\prime}, \ldots, \eta_{i}^{\prime}, \ldots, \eta_{N}^{\prime}\right) P\left(\eta_{1}^{\prime}, \ldots, \eta_{i}^{\prime}, \ldots, \eta_{N}^{\prime}, t\right)\right\}- \\
& \sum_{i}^{N} \sum_{\eta} \sum_{\eta^{\prime}}\left\{f\left(\eta_{1}, \ldots, \eta_{i}, \ldots, \eta_{N}, t\right) \delta\left(1-\eta_{i}^{\prime}, \eta_{i}\right)\right. \\
& \left.\prod_{\substack{j=1 \\
(j \neq i)}}^{N} \delta\left(\eta_{j}^{\prime}, \eta_{j}\right) w_{i}\left(\eta_{1}, \ldots, \eta_{i}, \ldots, \eta_{N}\right) P\left(\eta_{1}, \ldots, \eta_{i}, \ldots, \eta, t\right)\right\},
\end{aligned}
$$

em que $\eta^{i}$ (índice superescrito à esquerda) denota a configuração obtida de $\eta$ a partir de uma mudança anticíclica (conforme o esboço mostrado na figura 3.1). Tem-se, então, as transições: $(1 \rightarrow 0,0 \rightarrow 2,2 \rightarrow 1)$.

Como os únicos deltas de Kronecker não-nulos são $\delta\left(\eta_{i}, 1-\eta_{i}^{\prime}\right)=\delta\left(\eta_{i}, \eta_{i}^{i}\right)$ e $\delta\left(1-\eta_{i}, \eta_{i}^{\prime}\right)=\delta\left(\eta_{i}^{i}, \eta_{i}\right)$, pode-se escrever a equação anterior como se segue

$$
\begin{gathered}
\frac{d}{d t}<f(\eta)>=\sum_{i} \sum_{\eta} f(\eta) \omega_{i}\left(\eta^{i}\right) P\left(\eta^{i}, t\right)-f(\eta) \omega_{i}(\eta) P(\eta, t) \\
=\sum_{i}<f\left({ }^{i} \eta\right)-f(\eta) \omega_{i}(\eta)>
\end{gathered}
$$

A probabilidade de transição por sítio é definida a partir das regras definidas na seção 3.1, que podem ser escritas analiticamente por

$$
\omega_{i}(\eta)=c \delta\left(\eta_{i}, 2\right)+\frac{1}{\zeta} b \delta\left(\eta_{i}, 1\right) \sum_{\delta} \delta\left(\eta_{i+\delta}, 2\right),
$$

em que a soma é sobre os primeiros vizinhos do i-ésimo sítio.

Substituindo a probabilidade de transição por sítio, dada na equação (3.8), na equação (3.7) pode-se obter as equações de evolução temporal dos momentos da distribuição de probabilidades. 
Usando $f(\eta)=\delta\left(\eta_{i}, 1\right)$, pode-se definir a média $\left\langle\delta\left(\eta_{i}, 1\right)\right\rangle=P_{i}(1)$, que é a densidade de indivíduos suscetíveis, assim como $<\delta\left(\eta_{i}, 2\right)>=$ $P_{i}(2)$ representa a densidade de infectados, e $\left\langle\delta\left(\eta_{i}, 0\right)\right\rangle=P_{i}(0)$, que denota a densidade de recuperados. Quando se considera uma população constante, não há natalidade ou mortalidade, ficando implícita a condição de normalização,

$$
P(1)+P(2)+P(0)=1 .
$$

Assim, é suficiente calcular apenas dois dos primeiros momentos. O terceiro fica determinado pela condição de normalização.

Substituindo $f(\eta)=\delta\left(\eta_{i}, 1\right)$ na equação (3.7), tem-se:

$$
\begin{aligned}
\frac{d P_{i}(1)}{d t} & =<\left[\delta\left({ }^{i} \eta_{i}, 1\right)-\delta\left(\eta_{i}, 1\right)\right] \omega_{i}(\eta)> \\
& =<\left[\delta\left(\eta_{i}, 0\right)-\delta\left(\eta_{i}, 1\right)\right] \omega_{i}(\eta)> \\
& =<0-\frac{b}{\zeta} \delta\left(\eta_{i}, 1\right) \sum_{\delta} \delta\left(\eta_{i+\delta}, 2\right)> \\
& =-\frac{b}{\zeta} \sum_{\delta} P_{i, i+\delta}(12) .
\end{aligned}
$$

Nessa expressão, o somatório com índice $\delta$ é a contagem de primeiros vizinhos infectados; $\zeta$ é o número de coordenação da rede; e o termo $P_{i, i+\delta}(12)$ representa a probabilidade de haver um primeiro vizinho infectado ao lado de um indivíduo suscetível.

Repetindo os cálculos com $f(\eta)=\delta\left(\eta_{i}, 2\right)$, encontra-se:

$$
\begin{aligned}
\frac{d P_{i}(2)}{d t} & =<\left[\delta\left({ }^{i} \eta_{i}, 2\right)-\delta\left(\eta_{i}, 2\right)\right] \omega_{i}(\eta)> \\
& =<\left[\delta\left(\eta_{i}, 1\right)-\delta\left(\eta_{i}, 2\right)\right] \omega_{i}(\eta)> \\
& =<\frac{b}{\zeta} \delta\left(\eta_{i}, 1\right) \sum_{\delta} \delta\left(\eta_{i+\delta}, 2\right)-c \delta\left(\eta_{i}, 2\right)> \\
& =\frac{b}{\zeta} \sum_{\delta} P_{i, i+\delta}(12)-c P_{i}(2) .
\end{aligned}
$$

Utilizando as equações (3.9), (3.10) e (3.11), é possível também escrever a equação de evolução para $P_{i}(0)$,

$$
\frac{d}{d t} P_{i}(0)=-\frac{d}{d t} P_{i}(1)-\frac{d}{d t} P_{i}(2) .
$$




\subsection{Aproximação de Campo Médio Simples}

Em primeiro lugar, analisam-se as equações (3.10)-(3.12) por meio da aproximação de campo médio simples. Nesse caso, as correlações de dois ou mais sítios são escritas em termos das correlações de um sítio, isto é,

$$
P\left(\eta_{1}, \ldots, \eta_{i}, \ldots, \eta_{N}\right)=\prod P\left(\eta_{i}\right)
$$

Em particular a probabilidade conjunta $P_{i, i+\delta}(12)$ que aparece no lado direito das equações (3.10) e (3.11), é escrita na aproximação de campo médio simples como

$$
P_{i j}(\alpha \beta)=P_{i}(\alpha) P_{j}(\beta) .
$$

Com essa aproximação, assumindo invariância translacional e definindo as densidades $P(1)=x, P(2)=y$ e $P(0)=z$ que representam a densidade de indivíduos suscetíveis, infectados e recuperados, respectivamente, tem-se que as equações (3.10), (3.11) e (3.12) podem ser escritas como

$$
\begin{gathered}
\dot{x}=-b x y, \\
\dot{y}=b x y-c y,
\end{gathered}
$$

e

$$
\dot{z}=c y,
$$

assumindo-se que a rede seja isotrópica $P(12)=P(21)$.

Não foi possível obter a solução exata do sistema de equações (3.15)(3.17), entretanto, pelas equações (3.15)-(3.16) pode-se escrever a equação independente do tempo

$$
\frac{d y}{d x}=-1+\frac{c}{b} \frac{1}{x}
$$

Integrando a equação (3.18), obtem-se a seguinte equação:

$$
y+x-\frac{c}{b} \ln x=K,
$$

em que $K$ é a constante de integração. Essa equação expressa as " trajetórias" no espaço de fase. Ou seja, é uma equação de conservação entre as densidades. Como ela é uma equação independente do tempo, deve ser satisfeita em qualquer instante de tempo e, assim, permite-se usar as condições de contorno para determinar a solução estacionária.

Pelas hipóteses do modelo, no instante inicial $(t=0)$, a densidade de infectados tende a zero $(y \rightarrow 0)$ e a densidade de suscetíveis se aproxima do 
valor unitário $(x \rightarrow 1)$. Usando os valores $(x, y)=(1,0)$ na equação (3.19), obtem-se $K=1$, cujo valor é válido para qualquer instante $t$. No limite de tempo muito grande $(t \rightarrow \infty)$, a densidade de suscetíveis assume o valor assintótico $x=1-z_{\infty}$, em que $z_{\infty}$ é a densidade de indivíduos recuperados. Substituindo esses valores na equação (3.19) $\operatorname{com} K=1$ e expandindo a expressão em série de Taylor, trucando a série em termos de segunda ordem, obtem-se a solução estacionária

$$
z_{\infty}\left[2 c(c-b)+b^{2} z_{\infty}\right]=0 .
$$

Pela equação (3.20), nota-se que há duas raízes: $z_{\infty}=0$ e $z_{\infty}=\frac{b^{2}}{2 c(b-c)}$. A primeira corresponde à fase sem propagação da epidemia e a segunda, à fase com propagação da epidemia.

Pela equação (3.16), percebe-se que $x(t=0)>c / b$ é a condição necessária para que a densidade de infectados seja uma função crescente para tempos próximos de zero. Ou seja, na fase inicial de propagação da epidemia, deve-se ter $d y / d t>0$ (função crescente). Portanto, na aproximação de campo médio simples, $x_{0}=c / b$ é o limiar de espalhamento epidêmico.

O sistema de equações (3.15 3.17) apresenta os seguintes pontos fixos: $\left(x^{*}, y^{*}, z^{*}\right)=(1,0,0)$, que é marginalmente estável se $c>b$, e $\left(x^{*}, y^{*}, z^{*}\right)=$ $\left(x_{1}, 0, z_{1}\right)$. O primeiro ponto fixo representa o estado absorvente de suscetíveis, enquanto o segundo representa o estado absorvente de recuperados e suscetíveis. A perda de estabilidade ocorre quando $c=b$. Isso significa que, para $c>b$, ou seja, $c>1 / 2$, a epidemia não se espalha e toda a população se mantém suscetível. À medida que a probabilidade de imunização $c$ diminui (a probabilidade de infecção $b$ aumenta) tomando valores menores que o limiar $c_{c}=1 / 2$, parte da população torna-se infectada e, no estado estacionário, devido ao processo de recuperação espontânea, todos os indivíduos infectados tornam-se recuperados e imunes. Consequentemente, o sistema fica preso em um estado absorvente de suscetíveis e recuperados.

Na figura 3.2, consta a evolução das densidades de suscetíveis, infectados e recuperados em função do tempo. A densidade inicial de infectados é muito pequena (adotando $y(0)=10^{-4}$ ) e pode ser vista como uma perturbação, mas, como $c<c_{c}$, essa perturbação é suficiente para infectar uma parcela significativa da população. O processo de infecção cessa devido à imunização de todos os infectados. Como os processos de infecção e recuperação ocorrem concomitantemente, em determinado momento, prevalece o processo de imunização e o sistema vai para um estado absorvente. As curvas foram obtidas pela iteração das equações (3.15) 3.17) com o método de Runge-Kutta de quarta ordem.

Na figura 3.3, é possível observar a densidade estacionária de indivíduos recuperados em função da probabilidade de imunização (recuperação) $c$, na 


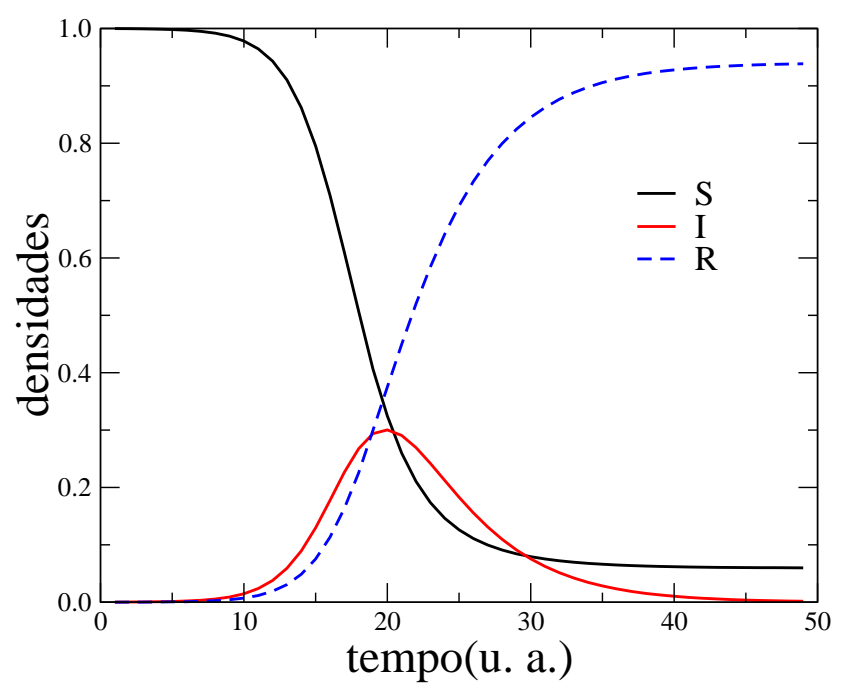

Figura 3.2: Curvas de evolução das densidades com $c=0,25$ na aproximação de campo médio simples para a densidade de suscetíveis $S$, infectados $I$ e recuperados $R$ em função do tempo.

aproximação de campo médio simples. Para fazer a integração numérica das equações (3.15 3.17) inicia-se com uma densidade muito baixa de indivíduos infectados $y \rightarrow 0$ e $x \rightarrow 1$ e, a cada iteração, resolve-se o sistema de equações diferenciais numericamente até as densidades atingirem um valor estacionário. Utilizando sempre a mesma condição inicial e variando a probabilidade de imunização $c$, calcula-se a densidade estacionária de indivíduos recuperados. Abaixo de $c_{c}=1 / 2$, há uma fase com espalhamento da epidemia e, por isso, a densidade de indivíduos recuperados é sempre maior que zero $R>0$. Acima do ponto crítico $c>1 / 2$, não há espalhamento da epidemia e, por isso, a densidade de indivíduos recuperados é nula $R=0$. A linha vertical tracejada separa a fase "ativa", na qual a epidemia se espalha, da fase "inativa" em que não há espalhamento da epidemia. 


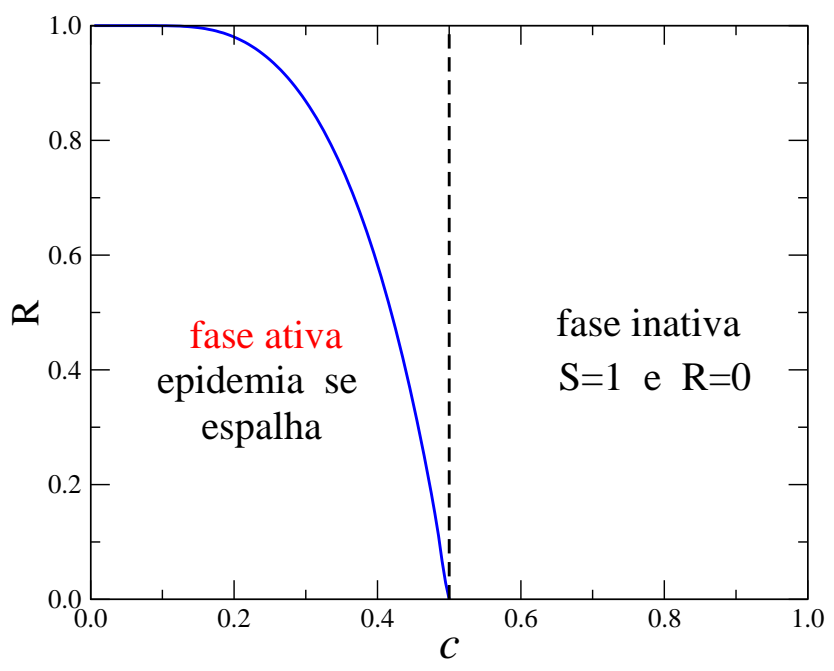

Figura 3.3: Densidade estacionária de indivíduos recuperados versus $c$ na aproximação de campo médio simples. 


\subsection{Aproximação de Campo Médio por Pares}

Ao calcular os primeiros momentos da distribuição, nota-se a dependência dos segundos momentos que, na aproximação de campo médio simples, foram descorrelacionados, a fim de obter em uma primeira aproximação do resultado exato (desconhecido). Para melhorar a solução de campo médio, deve-se considerar momentos de ordem maior. Para incluir os momentos de segunda ordem (correlações de dois sítios), não é necessário calcular todas as probabilidades conjuntas, pois há as seguintes condições de normalização:

$$
\begin{array}{r}
P(00)+P(01)+P(02)=P(0) \\
P(10)+P(11)+P(12)=P(1) \\
P(20)+P(21)+P(22)=P(2) \\
\mathrm{e} \quad P(0)+P(1)+P(2)=1 .
\end{array}
$$

Com a escolha de $P(1), P(2), P(01), P(02)$ e $P(12)$ como probabilidades independentes, resta determinar as equações de evolução temporal para as correlações de dois sítios, visto que na seção 3.3 já foram determinadas as equações para $P(1)$ e $P(2)$.

Usando $f(\eta)=\delta\left(\eta_{i}, 0\right) \delta\left(\eta_{j}, 1\right)$ na equação (3.7) e definindo $<\delta\left(\eta_{i}, 0\right) \delta\left(\eta_{j}, 1\right)>=P_{i j}(01)$, obtem-se:

$$
\frac{d P_{i j}(01)}{d t}=\sum_{k=1}^{N}<\left[\delta\left({ }^{k} \eta_{i}, 0\right) \delta\left({ }^{k} \eta_{j}, 1\right)-\delta\left(\eta_{i}, 0\right) \delta\left(\eta_{j}, 1\right)\right] \omega_{k}(\eta)>.
$$

Na somatória em $k$, há três casos a considerar:

- 1) Quando $k \neq i$ com $k \neq j$, tem-se

$$
\delta\left({ }^{k} \eta_{i}, 0\right) \delta\left({ }^{k} \eta_{j}, 1\right)-\delta\left(\eta_{i}, 0\right) \delta\left(\eta_{j}, 1\right)=\delta\left(\eta_{i}, 0\right) \delta\left(\eta_{j}, 1\right)-\delta\left(\eta_{i}, 0\right) \delta\left(\eta_{j}, 1\right)=0 .
$$

- 2) Quando $k=i$ com $k \neq j$, tem-se:

$$
\begin{array}{r}
\delta\left({ }^{k} \eta_{i}, 0\right) \delta\left({ }^{k} \eta_{j}, 1\right)-\delta\left(\eta_{i}, 0\right) \delta\left(\eta_{j}, 1\right)=\delta\left(\eta_{i}, 2\right) \delta\left(\eta_{j}, 1\right)-\delta\left(\eta_{i}, 0\right) \delta\left(\eta_{j}, 1\right) \\
<\left[\delta\left(\eta_{i}, 2\right) \delta\left(\eta_{j}, 1\right)-\delta\left(\eta_{i}, 0\right) \delta\left(\eta_{j}, 1\right)\right] \omega_{i}(\eta)>= \\
<c \delta\left(\eta_{i}, 2\right) \delta\left(\eta_{j}, 1\right)-0>=c P_{i j}(12)
\end{array}
$$

- 3) Quando $k \neq i$ com $k=j$, tem-se:

$$
\begin{array}{r}
\delta\left({ }^{k} \eta_{i}, 0\right) \delta\left({ }^{k} \eta_{j}, 1\right)-\delta\left(\eta_{i}, 0\right) \delta\left(\eta_{j}, 1\right)=\delta\left(\eta_{i}, 0\right)\left[\delta\left(\eta_{j}, 0\right)-\delta\left(\eta_{j}, 1\right)\right] \\
<\left[\delta\left(\eta_{i}, 0\right)\left[\delta\left(\eta_{j}, 0\right)-\delta\left(\eta_{j}, 1\right)\right] \omega_{j}(\eta)>=\right. \\
<0-\frac{b}{\zeta} \sum_{\delta} \delta\left(\eta_{i}, 0\right) \delta\left(\eta_{j}, 1\right) \delta\left(\eta_{j+\delta}, 2\right)>=-\frac{b}{\zeta} \sum_{\delta} P_{i, j, j+\delta}(012) .
\end{array}
$$


Logo, ao realizar a somatória no índice $k$, deve-se encontrar

$$
\frac{d P_{i j}(01)}{d t}=c P_{i j}(21)-\frac{b}{\zeta} \sum_{\delta} P_{i, j, j+\delta}(012) .
$$

A soma nos primeiros vizinhos do sítio $j$ deve ser feita percorrendo todos os primeiros vizinhos, excluindo-se o sítio $i$. Para $\zeta=4$ (rede quadrada), tem-se um aglomerado com seis sítios vizinhos ao par $\eta_{i} \eta_{j}$, na forma

$$
P\left(\begin{array}{cccc} 
& \eta_{2} & \eta_{3} & \\
\eta_{1} & \eta_{i} & \eta_{j} & \eta_{4} \\
& \eta_{6} & \eta_{5} &
\end{array}\right)
$$

Considerando esse aglomerado, a somatória da equação (3.26) torna-se:

$$
\begin{aligned}
& \sum_{\delta} P_{i, j, j+\delta}(012)=\sum_{\delta}<\delta\left(\eta_{i}, 0\right) \delta\left(\eta_{j}, 1\right) \delta\left(\eta_{j+\delta}, 2\right)>= \\
& \sum_{\eta_{i}, \eta_{3}, \eta_{4}, \eta_{5}}<\left(\delta\left(\eta_{i}, 2\right)+\delta\left(\eta_{3}, 2\right)+\delta\left(\eta_{4}, 2\right)+\delta\left(\eta_{5}, 2\right)\right) P\left(\begin{array}{ccc} 
& \eta_{3} & \\
0 & 1 & \eta_{4} \\
& \eta_{5} &
\end{array}\right)> \\
& =0+\sum_{\eta_{3}, \eta_{4}, \eta_{5}}<\delta\left(\eta_{3}, 2\right) P\left(\begin{array}{ccc} 
& \eta_{3} & \\
0 & 1 & \eta_{4} \\
& \eta_{5} &
\end{array}\right)+\sum_{\eta_{3}, \eta_{4}, \eta_{5}} \delta\left(\eta_{4}, 2\right) P\left(\begin{array}{ccc} 
& \eta_{3} & \\
0 & 1 & \eta_{4} \\
& \eta_{5} &
\end{array}\right)+ \\
& +\sum_{\eta_{3}, \eta_{4}, \eta_{5}} \delta\left(\eta_{5}, 2\right) P\left(\begin{array}{ccc} 
& \eta_{3} & \\
0 & 1 & \eta_{4} \\
& \eta_{5} &
\end{array}\right)> \\
& =P\left(\begin{array}{ll} 
& 2 \\
0 & 1
\end{array}\right)+P(012)+P\left(\begin{array}{ll}
0 & 1 \\
& 2
\end{array}\right) .
\end{aligned}
$$

Portanto, considerando isotropia e homogeneidade espacial, qualquer que seja a coordenação da rede, o i-ésimo sítio não contribui para a soma. Assim, deve-se ter:

$$
\frac{d P_{i j}(01)}{d t}=c P_{i j}(21)-b \frac{\zeta-1}{\zeta} P_{i, j, j+\delta}(012)
$$

Com $f(\eta)=\delta\left(\eta_{i}, 1\right) \delta\left(\eta_{j}, 2\right)$ na equação (3.7), e definindo $<\delta\left(\eta_{i}, 1\right) \delta\left(\eta_{j}, 2\right)>=P_{i j}(12)$, obtem-se:

$$
\frac{d P_{i j}(12)}{d t}=\sum_{k=1}^{N}<\left[\delta\left({ }^{k} \eta_{i}, 1\right) \delta\left({ }^{k} \eta_{j}, 2\right)-\delta\left(\eta_{i}, 1\right) \delta\left(\eta_{j}, 2\right] \omega_{k}(\eta)>\right.
$$


Analisando todas as possibilidades de haver valor não-nulo na somatória em $k$ :

- 1) Quando $k \neq i$ com $k \neq j$, tem-se:

$$
\delta\left({ }^{k} \eta_{i}, 1\right) \delta\left({ }^{k} \eta_{j}, 2\right)-\delta\left(\eta_{i}, 1\right) \delta\left(\eta_{j}, 2\right)=\delta\left(\eta_{i}, 1\right) \delta\left(\eta_{j}, 2\right)-\delta\left(\eta_{i}, 1\right) \delta\left(\eta_{j}, 2\right)=0 .
$$

- 2) Quando $k=i$ com $k \neq j$, tem-se:

$$
\begin{aligned}
& \delta\left({ }^{k} \eta_{i}, 1\right) \delta\left({ }^{k} \eta_{j}, 2\right)-\delta\left(\eta_{i}, 1\right) \delta\left(\eta_{j}, 2\right)=\left[\delta\left(\eta_{i}, 0\right)-\delta\left(\eta_{i}, 1\right)\right] \delta\left(\eta_{j}, 2\right) \\
& <\left[\delta\left(\eta_{i}, 0\right)-\delta\left(\eta_{i}, 1\right)\right] \delta\left(\eta_{j}, 2\right) \omega_{i}(\eta)>= \\
& <0-\frac{b}{\zeta} \sum_{\delta} \delta\left(\eta_{i}, 1\right) \delta\left(\eta_{j}, 2\right) \delta\left(\eta_{i+\delta}, 2\right)>\text {. } \\
& \sum_{\delta}<\delta\left(\eta_{i}, 1\right) \delta\left(\eta_{j}, 2\right) \delta\left(\eta_{i+\delta}, 2\right)>= \\
& \sum_{\eta_{1}, \eta_{2}, \eta_{j}, \eta_{6}}<\left(\delta\left(\eta_{1}, 2\right)+\delta\left(\eta_{2}, 2\right)+\delta\left(\eta_{j}, 2\right)+\delta\left(\eta_{6}, 2\right)\right) P\left(\begin{array}{ccc} 
& \eta_{2} & \\
\eta_{1} & 1 & 2 \\
& \eta_{6} &
\end{array}\right)>. \\
& =P_{i j}(12)+\sum_{\eta_{1}, \eta_{2}, \eta_{6}} \delta\left(\eta_{1}, 2\right) P\left(\begin{array}{ccc} 
& \eta_{2} & \\
\eta_{1} & 1 & 2 \\
& \eta_{6} &
\end{array}\right)+\sum_{\eta_{1}, \eta_{2}, \eta_{6}} \delta\left(\eta_{2}, 2\right) P\left(\begin{array}{ccc} 
& \eta_{2} & \\
\eta_{1} & 1 & 2 \\
& \eta_{6} &
\end{array}\right)+ \\
& +\sum_{\eta_{1}, \eta_{2}, \eta_{6}} \delta\left(\eta_{6}, 2\right) P\left(\begin{array}{ccc} 
& \eta_{2} & \\
\eta_{1} & 1 & 2 \\
& \eta_{6} &
\end{array}\right) \\
& =P(12)+P(212)+P\left(\begin{array}{ll} 
& 2 \\
1 & 2
\end{array}\right)+P\left(\begin{array}{ll}
1 & 2 \\
2 &
\end{array}\right) \text {. }
\end{aligned}
$$

- 3) Quando $k \neq i$ com $k=j$, tem-se:

$$
\begin{array}{r}
\delta\left({ }^{k} \eta_{i}, 1\right) \delta\left({ }^{k} \eta_{j}, 2\right)-\delta\left(\eta_{i}, 1\right) \delta\left(\eta_{j}, 2\right)=\delta\left(\eta_{i}, 1\right)\left[\delta\left(\eta_{j}, 1\right)-\delta\left(\eta_{j}, 2\right)\right] \\
<\delta\left(\eta_{i}, 1\right)\left[\delta\left(\eta_{j}, 1\right)-\delta\left(\eta_{j}, 2\right)\right] \omega_{j}(\eta)>= \\
<\frac{b}{\zeta} \sum_{\delta} \delta\left(\eta_{i}, 1\right) \delta\left(\eta_{j}, 1\right) \delta\left(\eta_{j+\delta}, 2\right)-\delta\left(\eta_{i}, 1\right) \delta\left(\eta_{j}, 2\right)>
\end{array}
$$


Realizando a soma sobre os primeiros vizinhos,

$$
\begin{gathered}
\sum_{\delta}<\delta\left(\eta_{i}, 1\right) \delta\left(\eta_{j}, 1\right) \delta\left(\eta_{j+\delta}, 2\right)>= \\
\sum_{\eta_{i}, \eta_{3}, \eta_{4}, \eta_{5}}<\left(\delta\left(\eta_{i}, 2\right)+\delta\left(\eta_{3}, 2\right)+\delta\left(\eta_{4}, 2\right)+\delta\left(\eta_{5}, 2\right)\right) P\left(\begin{array}{ccc}
\eta_{3} & \\
1 & 1 & \eta_{4} \\
& \eta_{5}
\end{array}\right)> \\
=0+P(12)+\left(\begin{array}{ll}
1 & 1 \\
& 2
\end{array}\right)+P(112)+P\left(\begin{array}{ll}
1 & 1 \\
2 &
\end{array}\right) .
\end{gathered}
$$

Após realizar a somatória no índice $k$, deve-se encontrar

$$
\begin{aligned}
\frac{d P_{i j}(12)}{d t}= & -\frac{b}{\zeta}\left[P_{i j}(12)+(\zeta-1) P_{i+\delta i j}(212)\right]+ \\
& \frac{b}{\zeta}(\zeta-1) P_{i j j+\delta}(112)-c P_{i j}(12) .
\end{aligned}
$$

Para obter a equação de evolução temporal para $P(02)$, deve-se usar $f(\eta)=\delta\left(\eta_{i}, 0\right) \delta\left(\eta_{j}, 2\right)$ na equação (3.7), que resulta

$$
\begin{array}{r}
\frac{d P_{i j}(02)}{d t}=\sum_{k=1}^{N}<\left[\delta\left({ }^{k} \eta_{i}, 0\right) \delta\left({ }^{k} \eta_{j}, 2\right)-\delta\left(\eta_{i}, 0\right) \delta\left(\eta_{j}, 2\right] \omega_{k}(\eta)>\right. \\
=<\left[\delta\left(\eta_{i}, 2\right)-\delta\left(\eta_{i}, 0\right)\right] \delta\left(\eta_{j}, 2\right) \omega_{i}(\eta)+\delta\left(\eta_{i}, 0\right)\left[\delta\left(\eta_{j}, 1\right)-\delta\left(\eta_{j}, 2\right)\right] \omega_{j}(\eta)> \\
=<c \delta\left(\eta_{i}, 2\right) \delta\left(\eta_{j}, 2\right)-0+\frac{b}{z} \sum_{\delta} \delta\left(\eta_{i}, 0\right) \delta\left(\eta_{j}, 1\right) \delta\left(\eta_{j+\delta}, 2\right)-c \delta\left(\eta_{i}, 0\right) \delta\left(\eta_{j}, 2\right)>
\end{array}
$$

Portanto,

$$
\frac{d P_{i j}(02)}{d t}=c P_{i j}(22)+b \frac{\zeta-1}{\zeta} P_{i j j+\delta}(012)-c P_{i j}(02)
$$

As equações para os segundos momentos da distribuição dependem de momentos de terceira ordem. Fazer a aproximação de campo médio por pares corresponde a truncar a dependência das correlações entre probabilidades em segunda ordem. Para isso, as correlações de trios são aproximadas da seguinte forma:

$$
P(\alpha \beta \gamma)=\frac{P(\alpha \beta) P(\beta \gamma)}{P(\beta)} .
$$

Com essa aproximação, e usando $x=P(1), \quad y=P(2), \quad u=P(01)$, $v=P(12)$ e $w=P(02)$, pode-se escrever as equações (3.10), (3.11), 
(3.27), (3.29) e (3.30) como:

$$
\begin{array}{r}
\dot{x}=-b v, \\
\dot{y}=b v-c y, \\
\dot{u}=-b \frac{\zeta-1}{\zeta} \frac{u v}{x}+c v, \\
\dot{v}=b \frac{\zeta-1}{\zeta} \frac{x-u-2 v}{x} v-\left(\frac{b}{\zeta}+c\right) v, \\
\dot{\mathrm{w}}=b \frac{\zeta-1}{\zeta} \frac{u v}{x}+c(y-v-2 w) .
\end{array}
$$

Um dos pontos fixos desse sistema de equações é $(x, y, u, v, w)=(1,0,0,0,0)$, que corresponde ao estado absorvente de suscetíveis. Fazendo a análise de estabilidade linear, é possível notar que esse ponto é marginalmente estável se $c>\frac{\zeta-2}{2(\zeta-1)}$. Quando $c=c_{c}=\frac{\zeta-2}{2(\zeta-1)}$, similar ao comportamento que ocorre na transição encontrada na aproximação de campo médio simples, o sistema perde a estabilidade. Abaixo desse limiar, a epidemia atinge parte da população mas, decorrido um tempo suficientemente longo, o sistema atinge um estado estacionário absorvente de suscetíveis e recuperados.

$\mathrm{Na}$ figura 3.4, consta a evolução das densidades indivíduos suscetíveis, infectados e recuperados/imunes na aproximação de campo médio por pares. As curvas foram obtidas pela iteração numérica das equações (3.10), (3.11), (3.27), (3.29) ) e (3.30), a partir de uma densidade inicial de infectados igual a $10^{-4}$.

Na figura 3.5, descreve-se a densidade estacionária de indivíduos recuperados em função da probabilidade de imunização para $(\zeta=4)$ (correspondente a rede quadrada regular). Nessa figura, constam os resultados de campo médio simples e campo médio por pares. Percebe-se que a aproximação de pares prevê que o regime onde há espalhamento de epidemia apresenta uma redução quando são consideradas correlações de maior ordem. Esse comportamento deve ser visto quando se faz também uma aproximação de trios. Contudo, a aproximação de campo médio é útil para estimar o comportamento do sistema e ter uma expectativa de como é o diagrama de fases. Para analisar como se comporta as grandezas médias na criticalidade, deve-se recorrer a outros métodos de investigação. Para este fim, serão realizadas simulações computacionais de Monte Carlo do modelo definido em uma rede regular, buscando determinar o ponto crítico e estudar o comportamento de grandezas de estado próximas da criticalidade. 


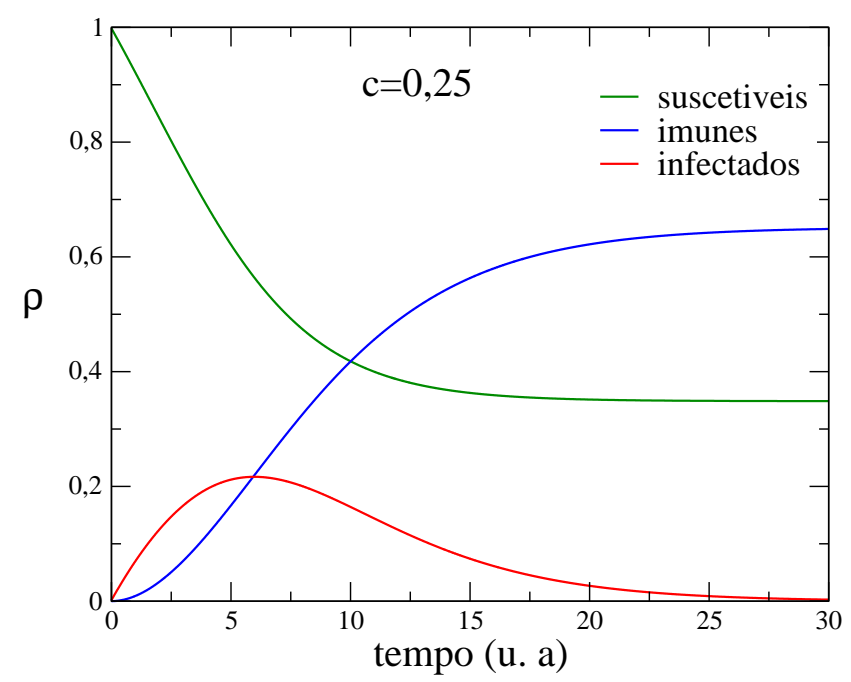

Figura 3.4: Curvas de evolução das densidades com $c=0,25$ na aproximação de campo médio por pares. As curvas foram obtidas pela iteração das equações (3.10), (3.11), (3.27), (3.29) ) e (3.30) $\operatorname{com} \zeta=4$ correspondente a uma rede regular quadrada.

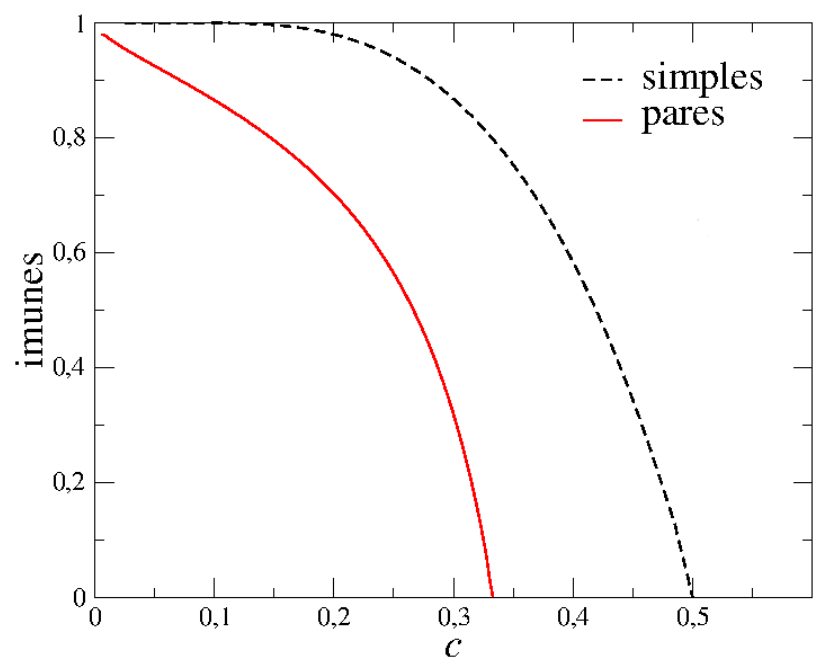

Figura 3.5: Densidade de recuperados versus a probabilidade de imunização c obtida por meio de aproximação de campo médio simples (linha tracejada) e por meio de aproximação de pares (linha contínua). Na aproximação de pares, fixa-se $\zeta=4$, que corresponde a uma rede quadrada. 


\subsection{Simulações de Monte Carlo estacionárias}

Com o método de Monte Carlo, é possível construir um algoritmo para realizar simulações computacionais do modelo SIR. É importante ressaltar que, em um sistema finito, esse modelo sempre evolui para um estado absorvente. E, assim, diferentemente de modelos com um único estado absorvente, não se pode calcular médias estacionárias usando uma média temporal sobre uma única realização. Todas as grandezas médias devem ser calculadas usando um conjunto de realizações e cada realização evolui até atingir um estado absorvente, então, a corrida temporal é interrompida e é computado o valor de cada uma das grandezas a ser analisadas. Isto é feito para um número amplo de realizações, com condições iniciais iguais, mas cada realização evolui independentemente.

Com base nas regras de transição do modelo, construiu-se uma primeira versão de algoritmo para simulação, o qual segue exatamente as etapas de evolução do modelo, cujos passos são:

- 1) Definir e inicializar uma rede regular com condições periódicas de contorno. Colocar todos os sítios no estado $\eta_{i}=1$ (suscetível), exceto o sítio central, com $\eta_{i}=2$ (infectado).

- 2) Sortear um sítio da rede e um número aleatório $\alpha(0 \leq \alpha<1)$. Efetuar o cálculo da probabilidade de transição do sítio $\omega_{i}(\eta)$ usando a equação (3.8). Se $\alpha \leqslant \omega_{i}(\eta)$, então o sítio assume seu próximo estado cíclico (segundo o diagrama apresentado na figura 3.1).

- 3) Repetir o passo 2 até que o último indivíduo infectado torne-se recuperado. Quando isso acontecer o sistema fica preso em um estado absorvente e a corrida é interrompida.

- 4) Calcular as grandezas médias.

Caso seja necessário definir uma unidade de tempo, introduz-se no passo 3 um critério de incremento temporal. Para cada $N$ sítios sorteados, em que $N$ é o total de sítios da rede, o tempo sofre acréscimo de uma unidade ou passo de Monte Carlo.

Uma vez que a simulação inicia-se com um único infectado, os sítios sorteados não terão seus estados modificados por tratar-se de sítios no estado suscetível sem primeiros vizinhos infectados. Isso ocorre principalmente no começo da simulação e acaba gerando ociosidade computacional. Pode-se contornar esse problema criando uma lista de sítios ativos, nos quais pode haver criação e aniquilação de infectados. Um algoritmo que permite simular o modelo SIR com maior eficiência computacional segue os seguintes passos: 
- 0) Para uma rede de $N$ sítios cria-se um vetor de dimensão $2 \times N$ (lista de sítios ativos), onde serão armazenadas as coordenadas dos sítios infectados. Inicializa-se todos os sítios da rede no estado 1 (suscetível), exceto o sítio central, que deve estar inicialmente no estado 2.

Para uma rede regular quadrada de lado $L$, adotando dois pontos (:) para designar toda a linha ou coluna de uma matriz ou vetor, tem-se:

$$
\begin{gathered}
\operatorname{rede}(:,:)=1 \\
\operatorname{rede}(L / 2, L / 2)=2 \\
\operatorname{La}(1,1)=L / 2 ; \operatorname{La}(1,2)=L / 2 \text { (lista de sítios infectados). } \\
N i=1 \text { (número de indivíduos infectados). }
\end{gathered}
$$

- 1) Sorteia-se um número aleatório $\alpha$ no intervalo $[0,1)$. Usa-se este número para escolher um sítio infectado. Para isto, faz-se $k=\operatorname{int}(1.0+$ $\alpha \times N i$ ), em que int denota a função inteiro, e o número $k$ irá indicar a posição deste sítio, a partir da lista de infectados. Capturam-se as coordenadas do sítio $i=L a(k, 1), j=L a(k, 2)$.

- 2) Sorteia-se um número aleatório $\beta(0 \leq \beta<1)$, que é comparado à probabilidade de recuperação $c$.

- 2.a) Se $\beta<c$, então, o indivíduo se recupera.

$$
\operatorname{rede}(i, j)=0
$$

reagrupa-se a lista de infectados para ficar compacta

$$
\begin{gathered}
L a(k,:)=L a(N i,:) \\
L a(N i,:)=0 \\
N i=N i-1 .
\end{gathered}
$$

- 2.b) Se $\beta \geqslant c$, então, sorteia-se um primeiro vizinho com coordenadas $(x, y)$. Se este vizinho for suscetível, torna-se infectado.

$$
\begin{gathered}
N i=N i+1 \\
L a(N i, 1)=x ; L a(N i, 2)=y \\
\operatorname{rede}(x, y)=2 .
\end{gathered}
$$

- 3) Enquanto há indivíduo infectado $(N i>0)$, repete-se o passo 2. Se $N i=0$, calculam-se as grandezas de estado.

As grandezas calculadas nas simulações estacionárias foram: número médio de indivíduos recuperados,

$$
S=<N_{R}>
$$


a densidade de indivíduos recuperados,

$$
\rho=\frac{<N_{R}>}{N}
$$

em que $N$ é o total de sítios da rede, o número quadrático médio de indivíduos recuperados,

$$
M=<N_{R}^{2}>,
$$

a razão entre o número quadrático médio de recuperados e o quadrado do número médio de recuperados,

$$
U=\frac{<N_{R}^{2}>}{<N_{R}>^{2}}=\frac{M}{S^{2}}
$$

e a probabilidade $P$ do aglomerado de recuperados atingir a borda mais próxima do centro da rede. Essa última grandeza foi definida como a fração do total de realizações em que há recuperados na borda da rede sobre o total de realizações. Mostrou-se que essa probabilidade é o parâmetro de ordem $P$ 44,46] e está relacionada à probabilidade de percolação. O número médio de indivíduos recuperados está relacionado ao tamanho médio dos aglomerados, e o número quadrático médio de indivíduos recuperados está associado ao terceiro momento da distribuição de aglomerados. Por isso, usaram-se as mesmas variáveis $S$ e $M$, calculadas para o modelo de percolação.

Foram realizadas várias simulações do modelo SIR definido em uma rede quadrada regular e também para o modelo SIR em uma rede triangular, cujos resultados serão apresentados nas próximas seções.

\subsection{Simulações na rede quadrada}

A rede quadrada regular possui coordenação $\zeta=4$ e é esperado que, em simulações computacionais de Monte Carlo, o ponto crítico $c_{c}$ seja menor que aquele obtido via aproximações de campo médio. Ressalta-se que o modelo possui somente um parâmetro de controle escolhido como a probabilidade de imunização $c, \operatorname{com} b=1-c$. Portanto, deve-se investigar como as grandezas médias variam em função desta probabilidade em redes de tamanho finito.

Nas figuras 3.6 3.9, constam retratos de uma realização para probabilidade de imunização $c=0,12 ; 0,15 ; 0,1765$ e 0,19 , respectivamente. Por elas verifica-se que, no estado estacionário, quanto menor o valor de $c$, maior a quantidade de indivíduos recuperados e o tamanho das "ilhas" de indivíduos suscetíveis formada pelos indivíduos que escaparam da epidemia crescem em função do aumento da probabilidade $c$. Abaixo do limiar de imunização $c_{c}$, 
que deve ser determinado, a epidemia se propaga e o aglomerado formado pelos indivíduos recuperados, na grande maioria das realizações, atinge a borda da rede. As simulações são executadas usando condições periódicas de contorno e a probabilidade $P$, que é o parâmetro de ordem do modelo, é definida como a probabilidade do aglomerado de recuperados atingir a face superior ou direita da rede (lados mais próximos do centro da rede).
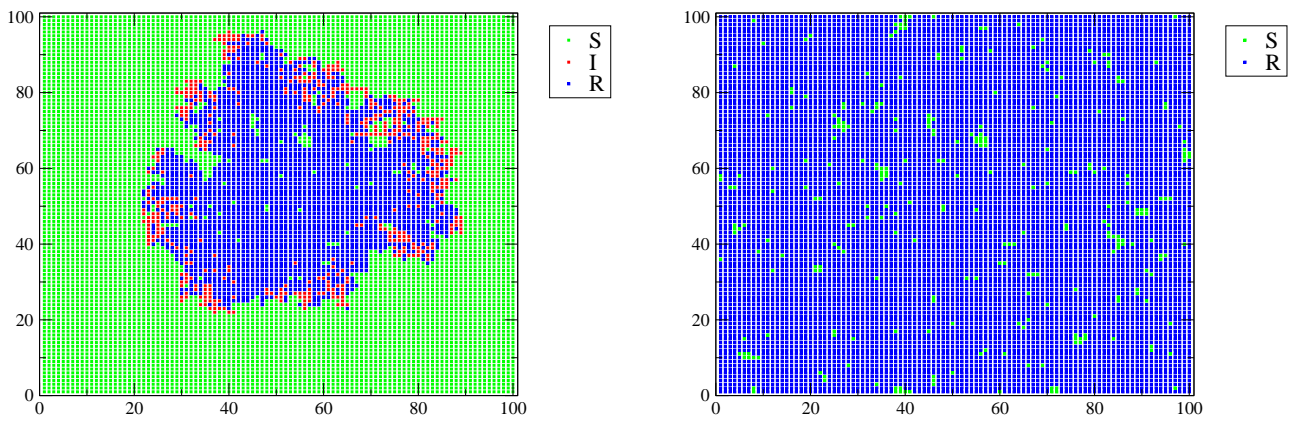

Figura 3.6: Retratos da rede com $L=100$ e $c=0,12$ durante a propagação da epidemia (instante $t=90$ ) e quando atinge o estado absorvente.
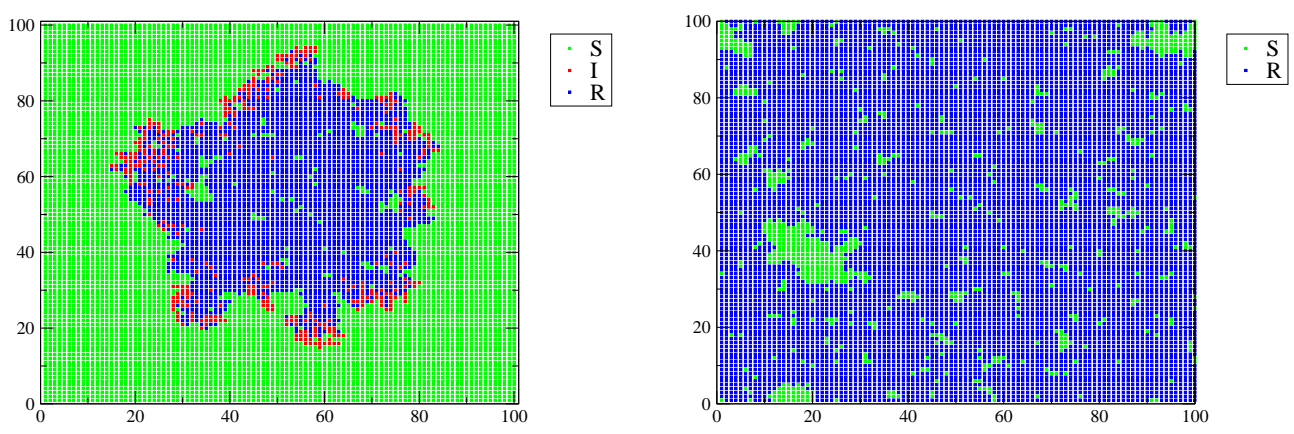

Figura 3.7: Retratos da rede com $L=100$ e $c=0,15$ durante a propagação da epidemia (instante $t=100$ ) e quando atinge o estado absorvente.

Os resultados estacionários apresentados nesta seção e na seção seguinte foram obtidos usando um conjunto entre $10^{7}$ e $10^{8}$ realizações independentes.

Na figura 3.10, consta a densidade estacionária de indivíduos recuperados em função da probabilidade de imunização para diferentes tamanhos de rede. Na figura 3.11, consta o parâmetro de ordem $P$ versus a probabilidade de imunização para diferentes tamanhos de rede. Na figura 3.10, observa-se que, para tamanhos grandes, aumentando a probabilidade de imunização, sai-se de uma fase com densidade não-nula de indivíduos recuperados e atingimos uma fase com densidade nula de indivíduos recuperados. Comportamento 

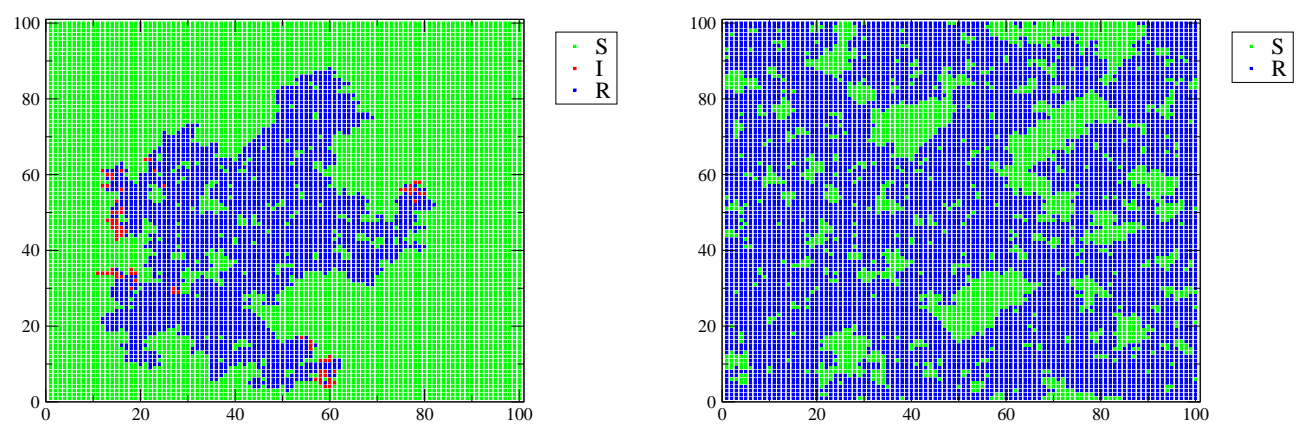

Figura 3.8: Retratos da rede com $L=100$ e $c=0,1765$ durante a propagação da epidemia (instante $t=180$ ) e quando atinge o estado absorvente.
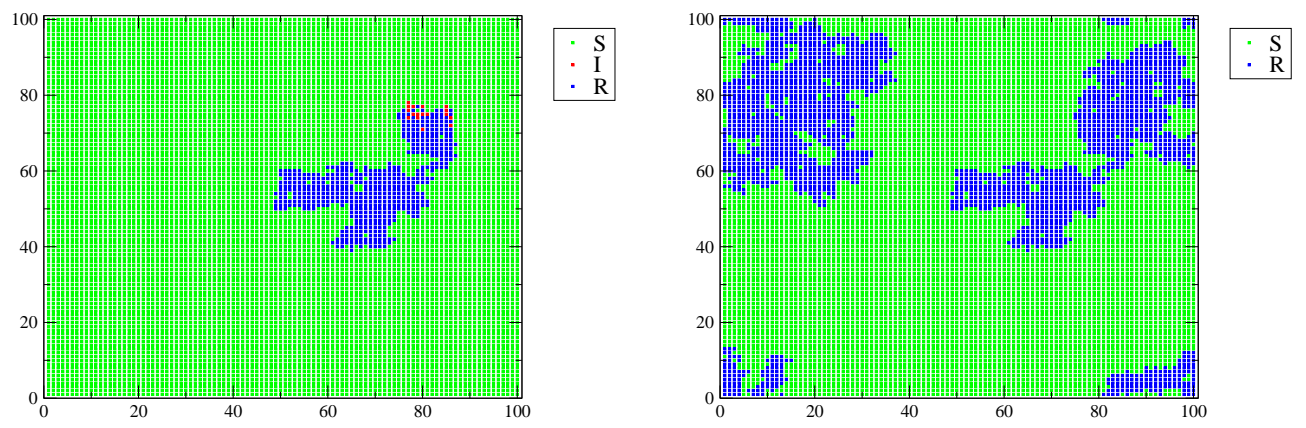

Figura 3.9: Retratos da rede com $L=100$ e $c=0,19$ durante a propagação da epidemia (instante $t=200$ ) e quando atinge o estado absorvente.

similar pode ser observado para o parâmetro de ordem $P$. Isso significa que, para um sistema de tamanho infinito, deve ocorrer uma transição de fase contínua.

A partir dessas figuras, não se pode afirmar com precisão qual deve ser o ponto crítico, mas fica nítido que para redes grandes a densidade de recuperados e o parâmetro de ordem se anulam acima deste limiar e, à medida que o tamanho da rede aumenta, mais bem definido fica este limiar.

O comportamento da razão entre os momentos $U=\frac{M}{S^{2}}$ definida na equação em 3.40 pode ser visto na figura 3.12 como função da probabilidade de imunização. A partir dessa figura, verifica-se que, no regime com espalhamento da epidemia, essa grandeza tende a um valor constante e, na proximidade do ponto crítico, $U$ cresce em função do aumento do tamanho da rede $L$. Não está representado nessa figura, mas muito acima do ponto crítico, $U$ volta a apresentar um mesmo valor para diferentes tamanhos de rede. Isso é distinto do comportamento observado em modelos com um único estado absorvente, como por exemplo o modelo de contato para o qual U, 


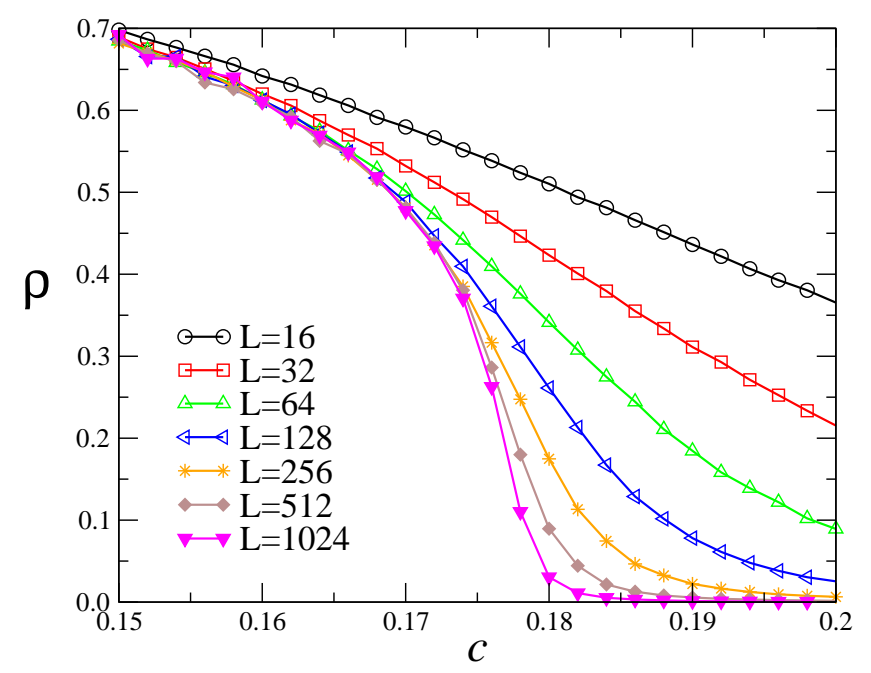

Figura 3.10: Densidade de indivíduos recuperados versus a probabilidade de imunização $c$ para diferentes tamanhos de rede quadrada de lado $L$.

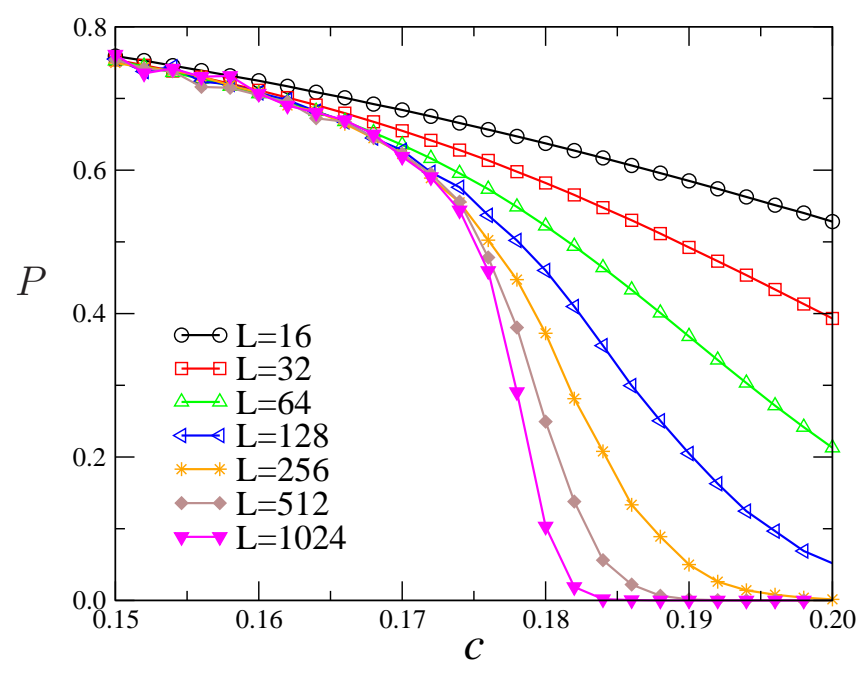

Figura 3.11: Probabilidade do aglomerado de indivíduos recuperados atingir a borda da rede $P$ versus a probabilidade de imunização $c$ para diferentes tamanhos de rede quadrada de lado $L$.

no ponto crítico, apresenta um valor universal (que independe do tamanho da rede). Neste modelo, à medida que o tamanho do sistema aumenta, a grandeza $U$ diverge e, por isso, deve-se procurar outra grandeza que seja universal no ponto crítico para que seja possível determinar o ponto crítico com praticidade e precisão. 


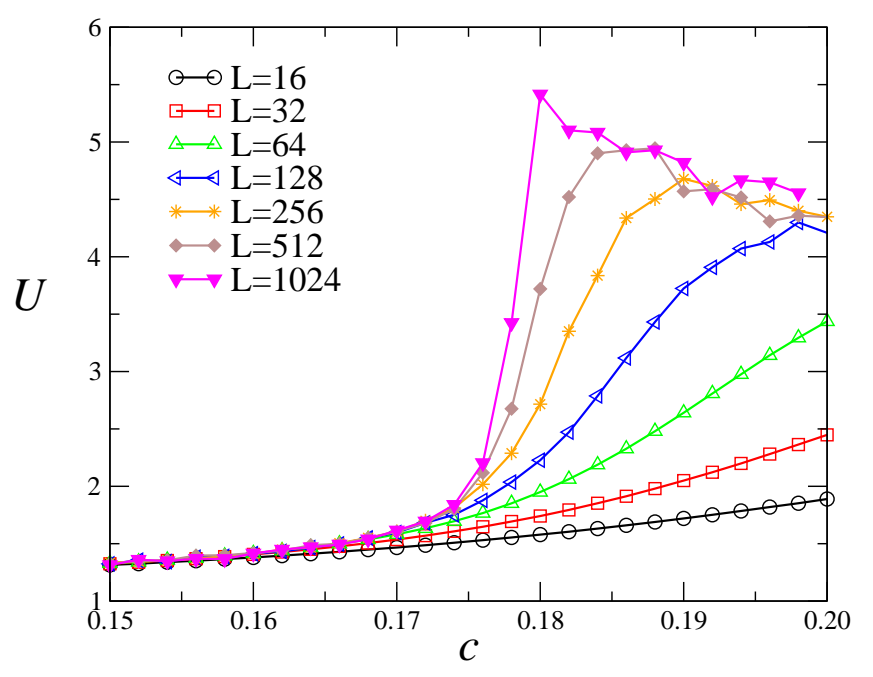

Figura 3.12: Razão entre o segundo e primeiro momento ao quadrado $U=\frac{M}{S^{2}}$ versus a probabilidade de imunização $c$ para diferentes tamanhos de rede quadrada de lado $L$.

\subsection{Comportamento crítico}

Para obter os expoentes críticos associados à transição de fase de nãoequilíbrio que ocorre no modelo SIR, serão analisadas esses dados por meio de simulações de Monte Carlo do modelo SIR definido em redes finitas de tamanho linear $L$. Constata-se, a partir da figura 3.10, que a densidade de indivíduos recuperados depende fortemente de $L$ nas vizinhanças da criticalidade. O mesmo ocorre para o parâmetro de ordem $P$ e para a razão $U$, como pode ser visto nas figuras 3.11 e 3.12 .

Partindo-se do pressuposto de que uma análise de escala finita pode ser feita para estudar a transição de fase de não-equilíbrio no modelo SIR, podese assumir que, para o sistema infinito, a transição é caracterizada por um comprimento de correlação $\xi$ que diverge no ponto crítico e possui o seguinte comportamento assintótico

$$
\xi \sim|\epsilon|^{-\nu_{\perp}},
$$

em que $\epsilon=c-c_{c}$ é o desvio do parâmetro de controle $c$ com relação ao valor crítico. Como nas proximidades do ponto crítico o tamanho linear do sistema $L$ se escala com o comprimento de correlação $\xi$, deve-se esperar que uma grandeza $A$ dependa de $L$ da seguinte maneira

$$
A_{L}(c)=L^{\alpha / \nu_{\perp}} F(L / \xi),
$$

$\mathrm{Ou}$

$$
A_{L}(c)=L^{\alpha / \nu_{\perp}} \widetilde{F}\left(L^{1 / \nu_{\perp}} \epsilon\right) .
$$


No limite em que $L \rightarrow \infty$, deve-se ter

$$
A_{\infty}(c) \sim|\epsilon|^{-\alpha} .
$$

Neste limite o parâmetro de ordem deve se comportar nas vizinhanças da criticalidade como

$$
P \sim|\epsilon|^{\beta},
$$

em que $\beta$ é o expoente crítico associado ao parâmetro de ordem. Portanto, de acordo com a expressão (3.43), o parâmetro de ordem deve obedecer à seguinte forma de escala

$$
P_{L}(c)=L^{-\beta / \nu_{\perp}} f\left(L^{1 / \nu_{\perp}} \epsilon\right),
$$

em que $f(x)$ é uma função universal.

As grandezas que caracterizam o modelo SIR podem ser relacionadas às grandezas da teoria de percolação. Pelas equações (2.64) e (2.69), tem-se

$$
S \sim|\epsilon|^{-\gamma},
$$

e

$$
M \sim|\epsilon|^{-\beta-2 \gamma} .
$$

Portanto,

$$
U \sim|\epsilon|^{-\beta} .
$$

Pelas expressões (3.44) e (3.47), o número médio de indivíduos recuperados deve obedecer à seguinte forma de escala

$$
S_{L}(c)=L^{\gamma / \nu_{\perp}} h\left(L^{1 / \nu_{\perp}} \epsilon\right),
$$

em que $h(x)$ é uma função universal. Pelo mesmo raciocínio, a partir das equações (3.44) e (3.48), tem-se

$$
M_{L}(c)=L^{(\beta+2 \gamma) / \nu_{\perp}} j\left(L^{1 / \nu_{\perp}} \epsilon\right),
$$

em que $j(x)$ é uma função universal. E a razão $U$ deve obedecer à forma de escala

$$
U_{L}(c)=L^{\beta / \nu_{\perp}} k\left(L^{1 / \nu_{\perp}} \epsilon\right) .
$$

No ponto crítico $\epsilon=0$, as grandezas que estão sendo investigadas para o modelo SIR obedecem às seguintes formas de escala:

$$
\begin{array}{r}
P \sim L^{-\beta / \nu_{\perp}}, \\
S \sim L^{\gamma / \nu_{\perp}}, \\
M \sim L^{(\beta+2 \gamma) / \nu_{\perp}},
\end{array}
$$


e

$$
U \sim L^{\beta / \nu_{\perp}}
$$

Veja que, por estas relações, o produto entre $U$ e $P$ torna-se independente do tamanho da rede $L$. Introduziu-se essa nova grandeza, denominada $U P$, como grandeza que estabelece um novo método para calcular o ponto crítico em simulações de Monte Carlo para o modelo SIR. Devido à independência com relação ao tamanho da rede $L$, em um gráfico de $U P$ versus $c$ para diferentes valores de $L$, deve haver um cruzamento das curvas no ponto crítico. Como pode ser visto na figura 3.13, isso de fato acontece. Usando esse método, a estimativa para $c_{c}$ é $0,17650(2)$, que está de acordo com os valores conhecidos [34,44]. Além disso, conforme pode ser visto figura 3.14, pelo cruzamento entre duas curvas sucessivas de $U P$ versus $1 / L$, é possível fazer uma extrapolação para um sistema de tamanho infinito $(1 / L \rightarrow 0)$, e obter o valor crítico de $U P=1,0167(1)$, o qual concorda com o valor de $U P$ obtido para a percolação por sítio e ligação [46].

No ponto crítico, $c=c_{c}$ e $\epsilon=0$, e a partir da equação (3.53) pode-se escrever

$$
\ln \left[P_{L}\left(c_{c}\right)\right]=-\beta / \nu_{\perp} \ln L+C,
$$

em que $C$ é uma constante. É possível obter a razão entre os expoentes $\beta / \nu_{\perp}$ a partir do gráfico de $\ln P_{l}\left(c_{c}\right)$ versus $\ln L$.

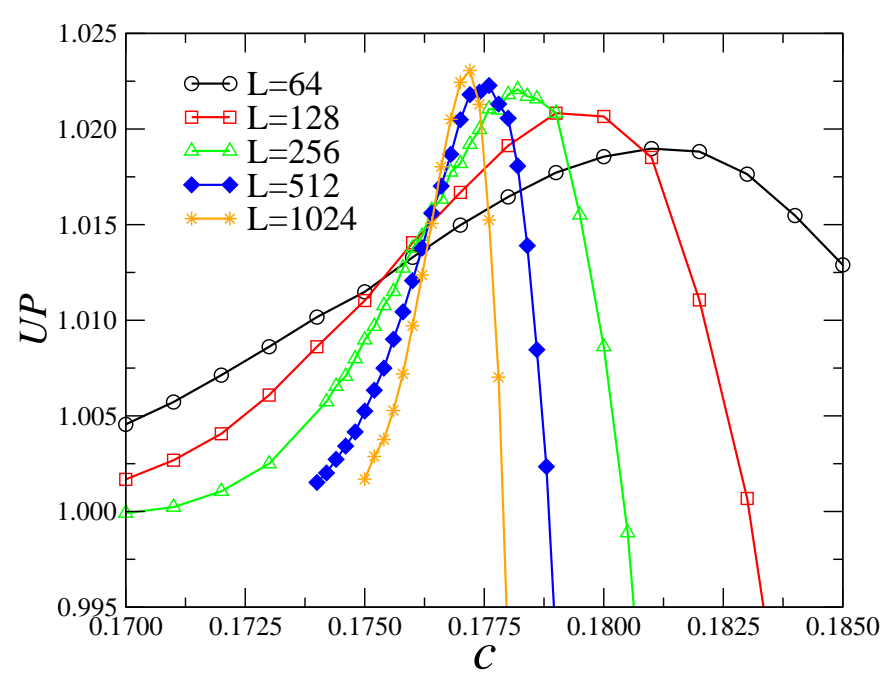

Figura 3.13: Produto da razão entre o segundo e primeiro momento $U=\frac{M}{S^{2}}$ pelo parâmetro de ordem $U P$ versus a probabilidade de imunização $c$ para diferentes tamanhos de rede quadrada de lado $L$. 


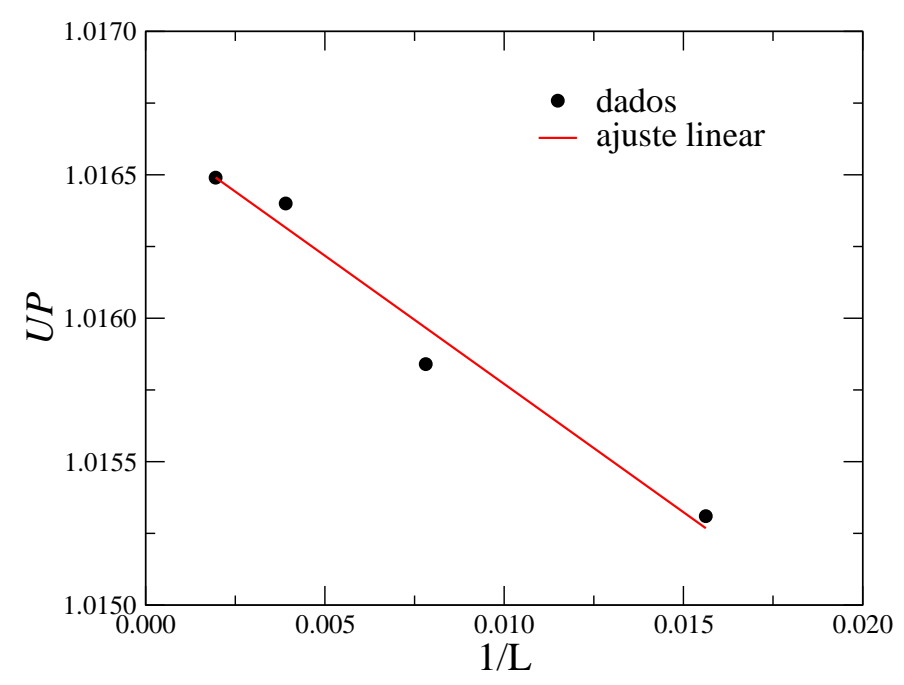

Figura 3.14: $U P$ versus $1 / L$, em que $U P$ é o valor obtido para o cruzamento das curvas mostrada na figura 3.13 para dois tamanhos consecutivos de rede de lado $L$.

Na figura 3.15, consta a densidade de indivíduos recuperados versus o tamanho da rede para diferentes probabilidades de imunização. No regime subcrítico $\left(c>c_{c}\right)$, para $L \rightarrow \infty$ a densidade de recuperados se anula e, assim, para redes finitas as curvas de $\rho$ em função de $L$ são convexas. Por outro lado, no regime supercrítico, mesmo para tamanho infinito, a densidade não vai a zero, pois assume um valor constante não-nulo. Em redes de tamanho finito, isso resulta em curvas côncavas para $\rho$ versus $L$. No ponto crítico, a densidade descresce segundo uma lei de potência. O parâmetro de ordem apresenta esse mesmo comportamento. Na figura 3.16, tem-se o parâmetro de ordem versus $L$, em escala log-log, para $c=0,17650$. O coeficiente linear é a razão $\beta / \nu_{\perp}=0,1048(10)$. Na figura 3.17, em escala log-log, consta o número médio de recuperados $S$ versus $L$ para $c_{c}=0,17650(2)$, cujo coeficiente linear permite calcular $\gamma / \nu_{\perp}=1,7923(5)$. Pode-se comparar estes resultados com os valores exatos $\beta / \nu_{\perp}=5 / 48=0.1042$ e $\gamma / \nu_{\perp}=43 / 24=1.792$ que são calculados com $\beta=5 / 36$ e $\nu_{\perp}=4 / 3$ da percolação em duas dimensões.

Para averiguar a validade da hipótese de escala finita usada para o modelo, deve-se verificar se, usando os expoentes críticos encontrados, é possível colapsar os dados de cada uma das grandezas em estudo. Na figura 3.18, tem-se o colapso para o parâmetro de ordem $P$ versus $c$ para diferentes tamanhos de rede quadrada de lado $L$. Percebe-se que, dentro do intervalo de probabilidades analisado, os dados colapsam, confirmando a validade de nossas suposições. 


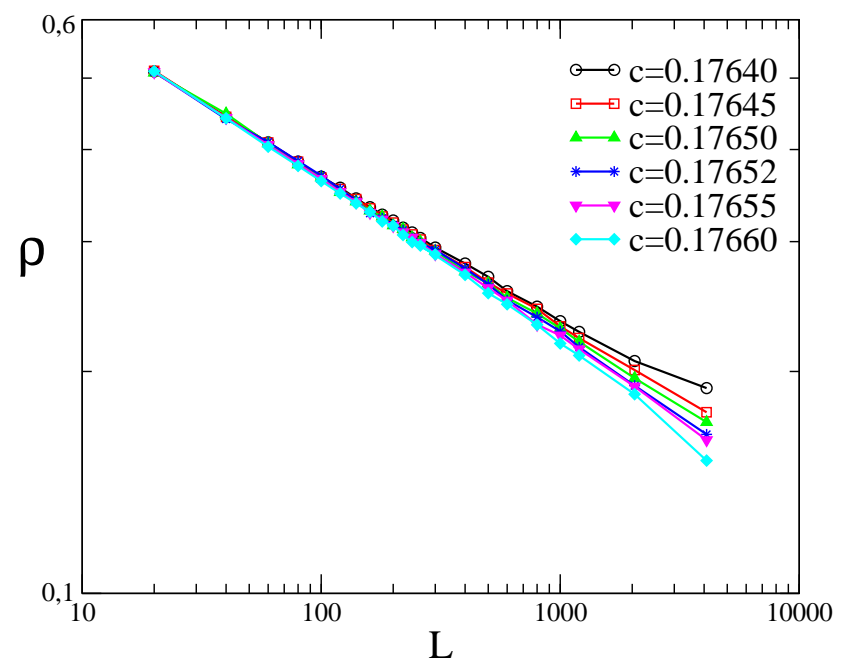

Figura 3.15: Densidade de recuperados versus o tamanho linear da rede $L$ para diferentes probabilidades de imunização $c$. A densidade se escala segundo a relação $\rho \sim L^{-2 \beta / \nu_{\perp}}$.

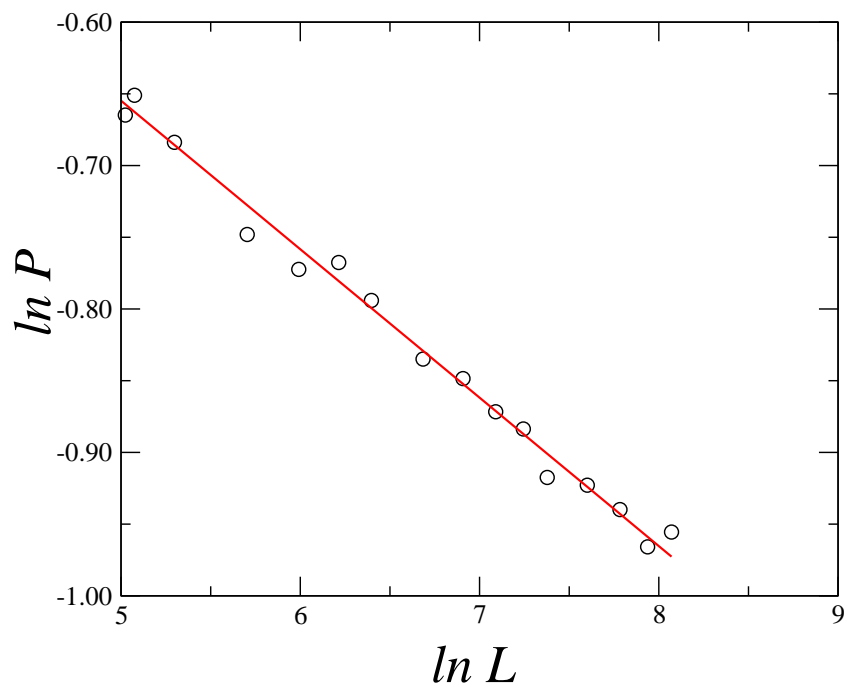

Figura 3.16: $\operatorname{Ln}(P)$ versus $\ln (L)$ para $c=0,17650$. A reta ajustada aos dados (linha contínua), conforme a equação (3.53), apresenta coeficiente linear $\beta / \nu_{\perp}=0,1048(10)$.

O colapso para o número médio de recuperados $S$ pode ser visto na figura 3.19 para diferentes valores de $L$, cujo comportamento está em concordância com o comportamento crítico dado pela equação (3.48).

Em vez de mostrar o colapso para a grandeza $U$, que deve escalar com o 


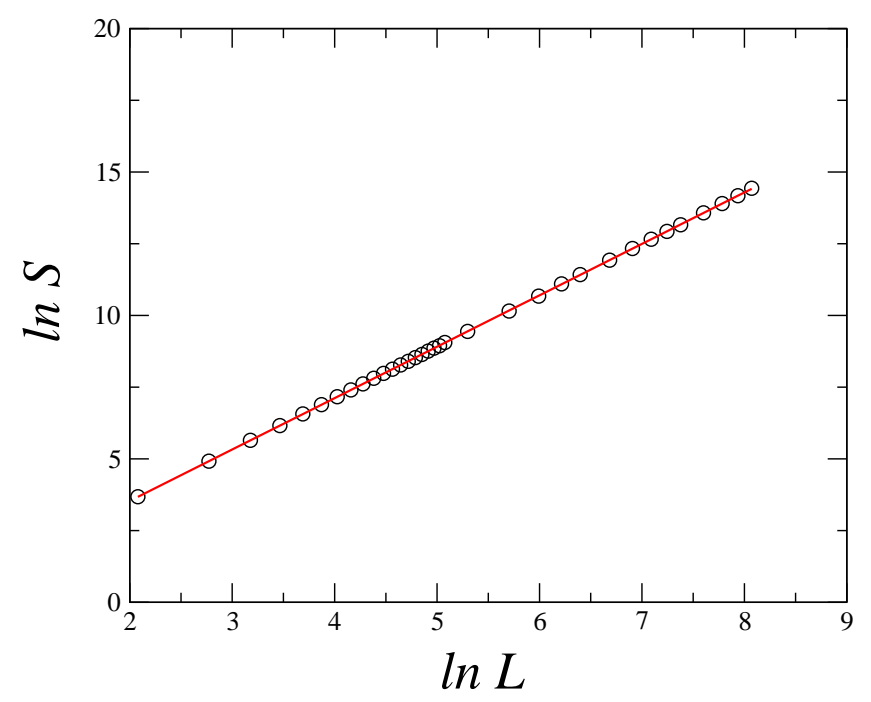

Figura 3.17: $\operatorname{Ln}(S)$ versus $\operatorname{Ln}(L)$. Ajustando uma reta aos dados (linha contínua), conforme a equação (3.57), obtemos $\gamma / \nu_{\perp}=1,723(5)$.

expoente $\beta / \nu_{\perp}$, o qual já foi verificado a partir da relação (3.47), conforme pode ser visto na figura 3.18, analisou-se a grandeza $U P$ que escala com expoente unitário. Na figura 3.20, está representado o colapso dos dados de $U P$ versus $c$ para diferentes tamanhos de rede de lado $L$. Os resultados são consistentes com as hipóteses feitas.

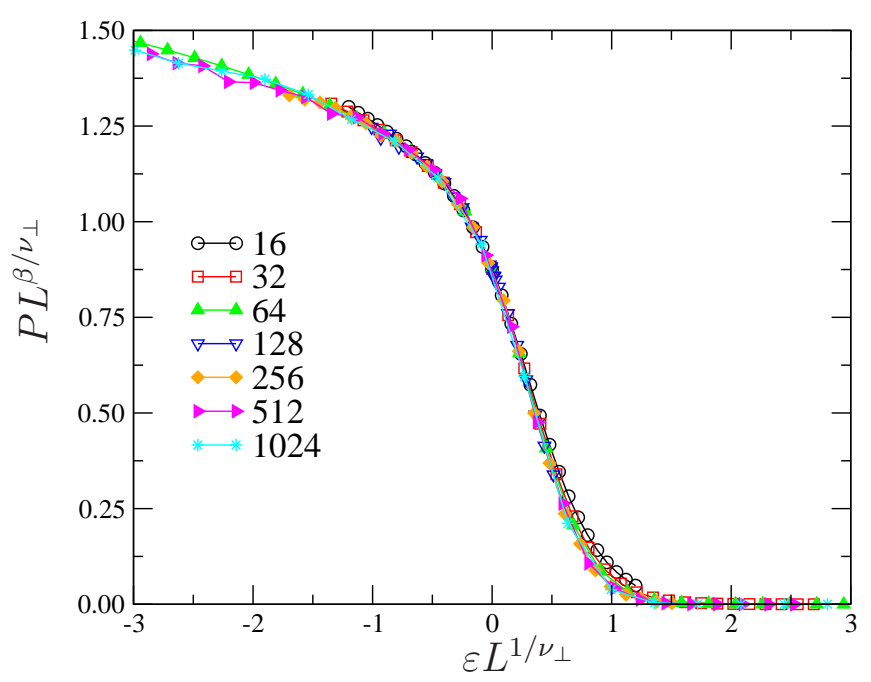

Figura 3.18: Colapso de dados de $P$ para diferentes valores de $L$. 


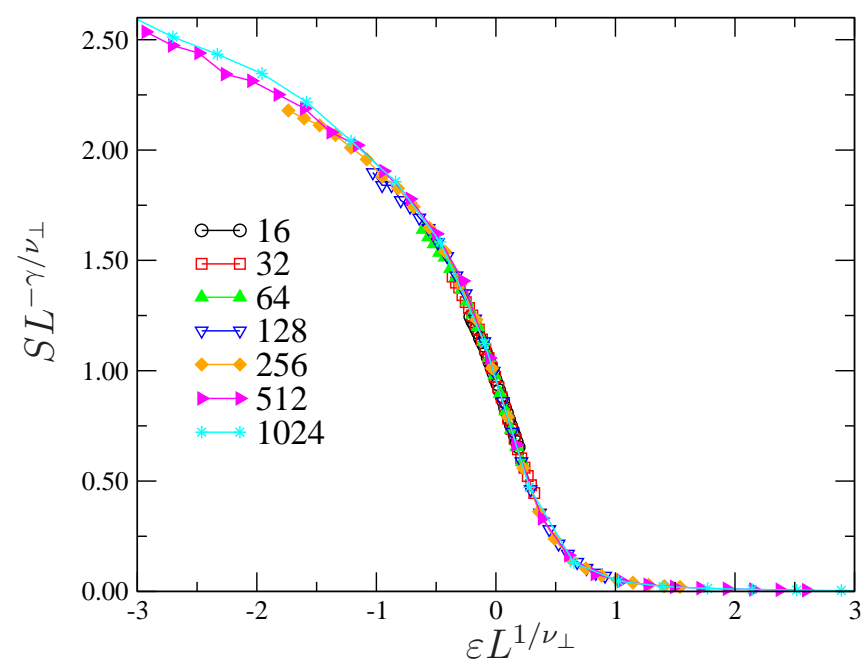

Figura 3.19: Colapso de dados de $S$ para diferentes valores de $L$.

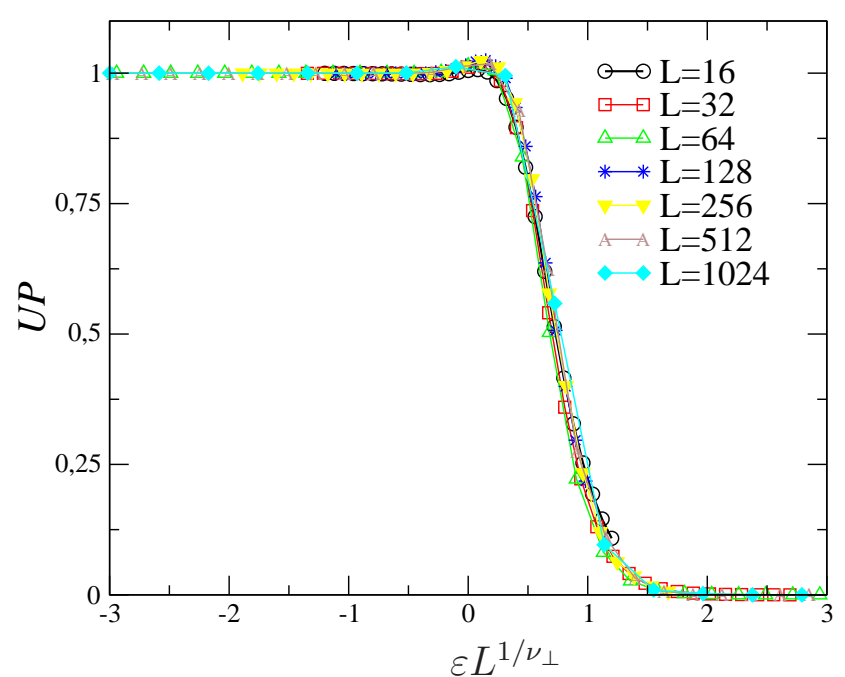

Figura 3.20: Colapso de dados de $U P$ para diferentes valores de $L$. 


\subsection{Simulações na rede triangular}

Nessa seção analisou-se o modelo SIR estocástico e espacialmente definido em uma rede triangular. Essa estrutura de rede, representada na figura 3.21. tem coordenação $\zeta=6$ e, conforme a aproximação de campo médio por pares para uma rede hipercúbica, o ponto crítico é $c_{c}=\frac{\zeta-2}{2(\zeta-1)}$. Substituindo $\zeta=6$ obtem-se $c_{c}=2 / 5$. Sabe-se que a aproximação de campo médio superestima o ponto crítico e, portanto, utilizaram-se simulações de Monte Carlo para determinar o ponto crítico e investigar o comportamento crítico de algumas grandezas que já foram analisadas para a rede quadrada.

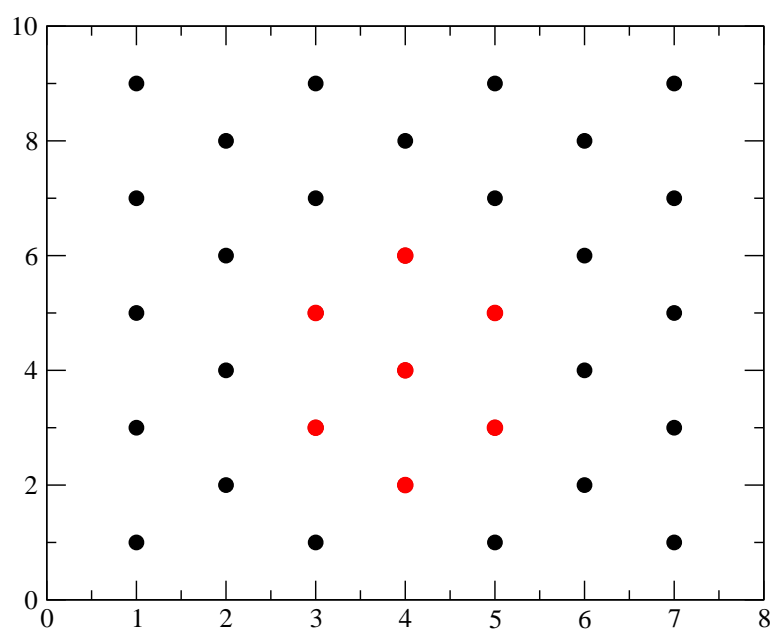

Figura 3.21: Estrutura da rede triangular. Nesta rede cada sítio tem seis sítios como primeiros vizinhos. Uma célula está destacada na cor vermelha.

Na figura 3.22, consta a densidade de indivíduos recuperados, a probabilidade $P$ da epidemia atingir a borda da rede, o cumulante $U$ e $o$ produto $U \times P$ em função da probabilidade de recuperação $c$ para diferentes tamanhos de rede. Estes resultados indicam que há uma transição de fase cujo ponto crítico está próximo de $c=0,20$. É possível determinar o ponto crítico usando o comportamento universal da grandeza UP. Entretanto, explorando a conexão entre o modelo SIR e o modelo de percolação, pode-se usar outro método para determinar o ponto crítico.

Como demonstrado na seção 2.5, é possível escolher os expoentes críticos $\beta$ e $\gamma$ ou $\sigma$ e $\tau$ como expoentes fundamentais. Logo, diferentemente do procedimento adotado nas simulações do modelo SIR na rede quadrada, tomando $\tau$ e $\sigma$ como expoentes fundamentais, também pode-se determinar o ponto crítico. Nesse caso, define-se a probabilidade acumulada de ter 

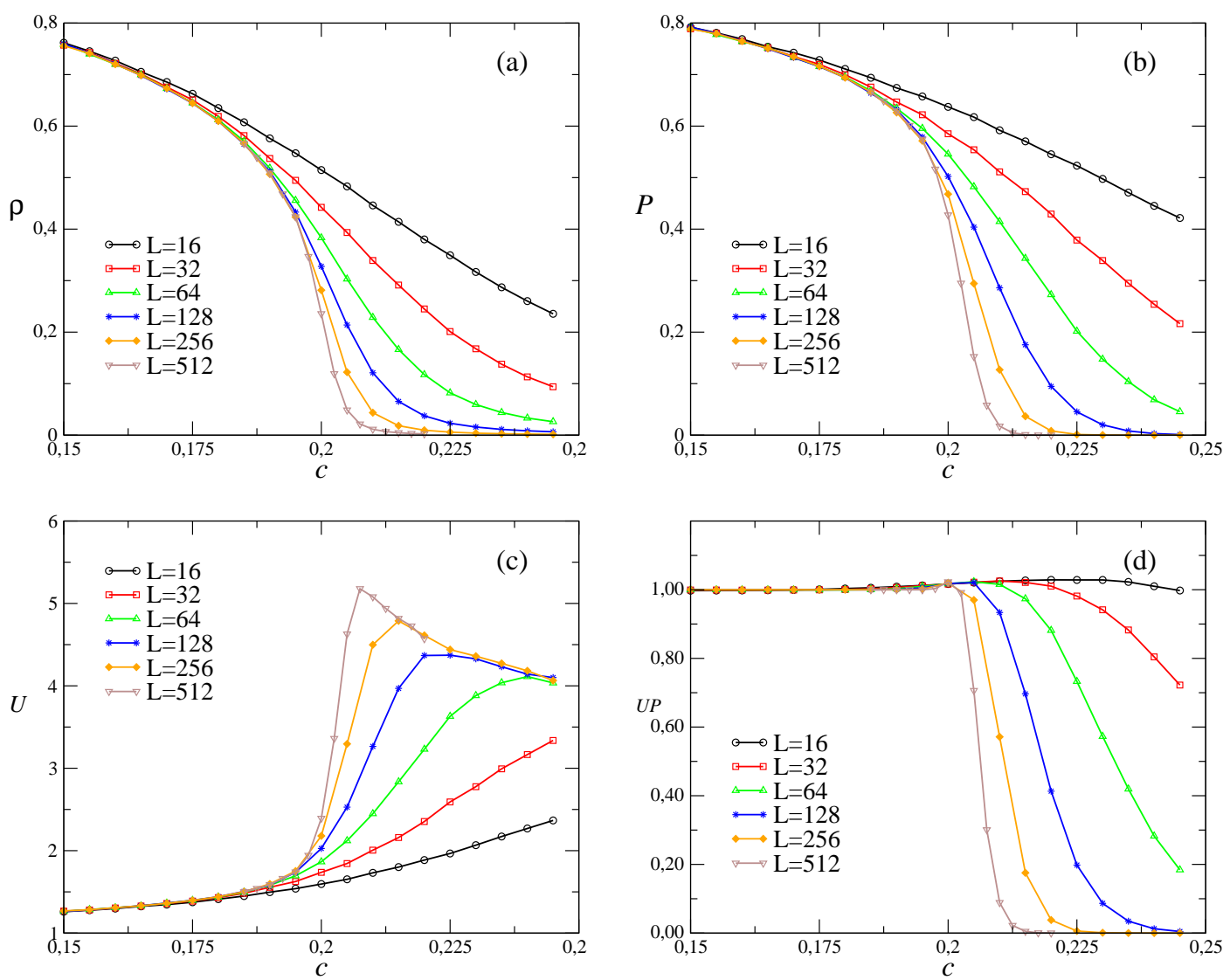

Figura 3.22: Em (a) densidade de recuperados, (b) probabilidade da epidemia atingir a borda da rede, (c) cumulante $U$, (d) UP versus a probabilidade de imunização para diferentes tamanhos da rede de lado $L$.

aglomerados de tamanho maior ou igual a um valor $s$ como,

$$
P_{\geq s}=\sum_{i=s}^{\infty} P(i),
$$

em que $P(i)$ é a probabilidade de encontrar um aglomerado com $i$ indivíduos recuperados no estado estacionário.

No ponto crítico $c_{c}$, espera-se que essa probabilidade acumulada obedeça à lei de potência

$$
P \sim \epsilon^{2-\tau} .
$$

Ou seja, que essa grandeza se comporte de acordo com a equação (2.71) proveniente da percolação usual. Além disso, espera-se que, nas vizinhanças da criticalidade e para $s$ suficientemente grande, essa probabilidade obedeça 
à forma de escala

$$
P_{\geq s}=s^{2-\tau} F\left(\epsilon s^{\sigma}\right),
$$

em que $\epsilon=c-c_{c}$ e $F(x)$ é uma função universal, ou seja, comporte-se de acordo com a equação (2.72) proveniente também da percolação. A expansão de $F(x)$ em série de Taylor para $c$ próximo de $c_{c}$ leva a seguinte expressão válida na região de escala [44,71]:

$$
P_{\geq s} s^{\tau-2} \sim c_{1}+c_{2} \epsilon s^{\sigma},
$$

em que $c_{1}$ e $c_{2}$ são constantes. Os expoentes $\tau$ e $\sigma$ para a percolação usual em duas dimensões são conhecidos exatamente: $\tau=187 / 91$ e $\sigma=36 / 91$. Assim, pode-se empregar estes valores na equação (3.61) e, a partir dela, determinar o ponto crítico. Nota-se que, usando somente termos de primeira ordem, a grandeza $P_{\geq s}$ torna-se independente do expoente crítico $\sigma$.

Na figura 3.23, consta $P_{\geq_{s}} s^{\tau-2}$ versus $s^{\sigma}$ para diferentes probabilidades de recuperação $c$. Na fase subcrítica $c>c_{c}$, as curvas tem inclinação negativa, enquanto na fase supercrítica a inclinação é positiva. No ponto crítico, a inclinação deve ser nula. Os coeficientes lineares de $P_{\geq_{s}} s^{\tau-2}$ são ilustrados na figura 3.24 em função de $c$. Ajustando uma reta a estes dados, é possível determinar o valor de $c$ tal que a curva de $P_{\geq s} s^{\tau-2}$ tenha coeficiente linear nulo. Pela reta ajustada aos dados, obtem-se $c_{c}=0,199727(6)$. Conforme discutido na referência [44] esse método possibilita obter o ponto crítico com precisão de aproximadamente 200 vezes maior que usando simulações dependentes do tempo ou simulações estacionárias (calculando a grandeza $U P$ ). Pode-se aplicar esse mesmo método para investigar o modelo definido em outras estruturas regulares, inclusive na rede quadrada, como pode ser visto na referência 44] e no modelo de percolação [71]. 


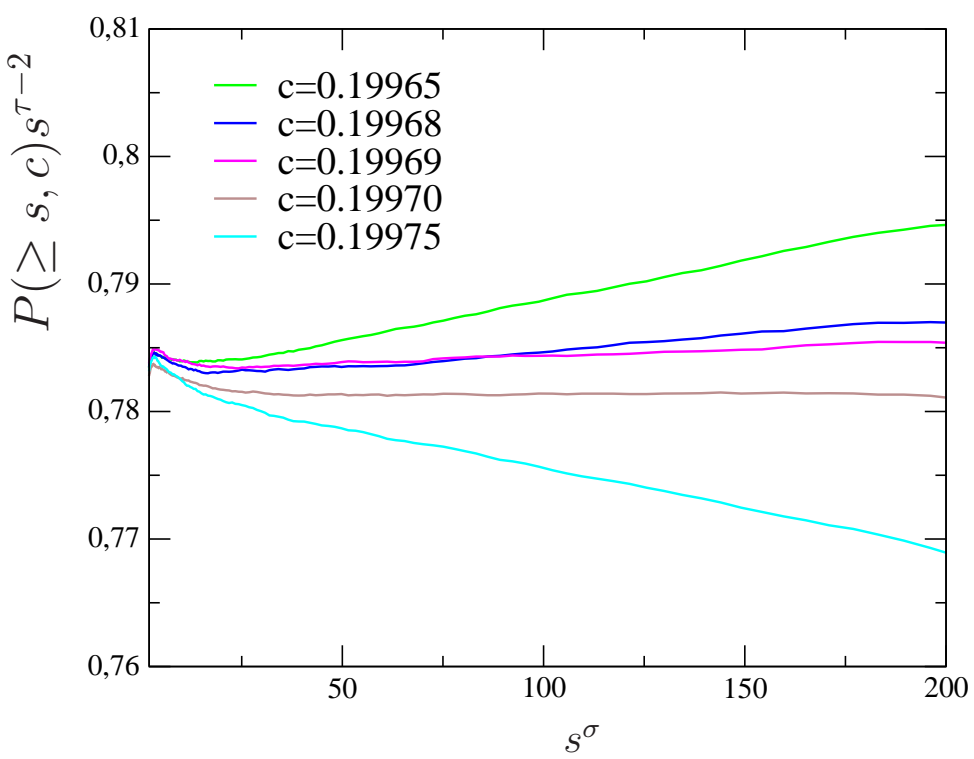

Figura 3.23: Probabilidade acumulada de encontrar aglomerados com mais de $s$ recuperados multiplicada pelo tamanho dos aglomerados reescalada com o expoente $\tau-2$ versus $s^{\sigma}$.

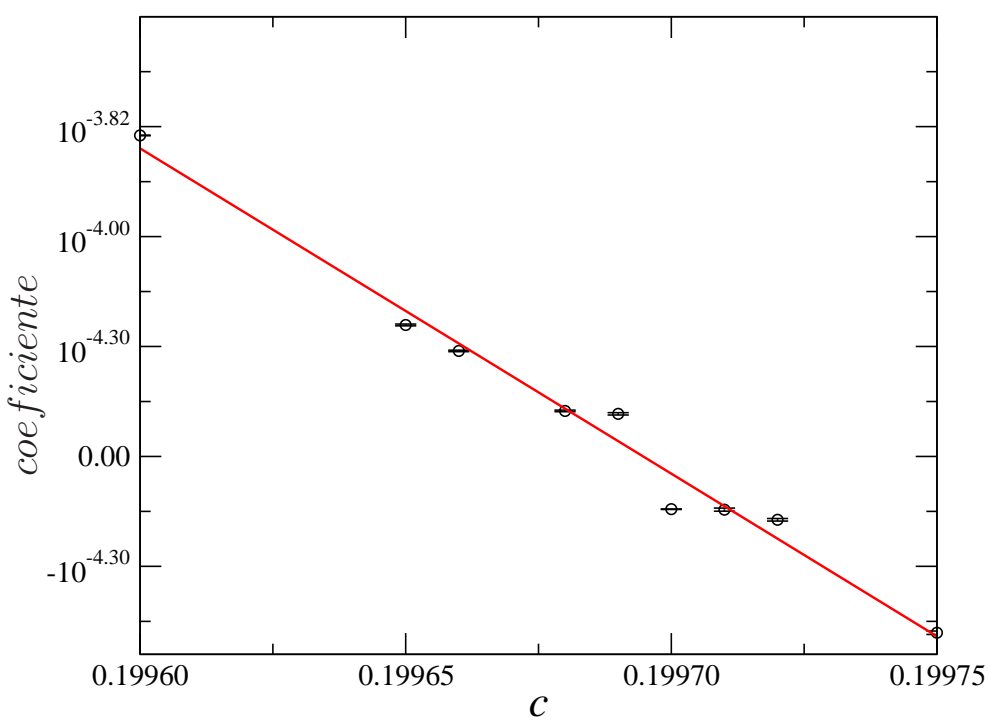

Figura 3.24: Coeficientes lineares das retas ajustadas às curvas mostradas na figura 3.23 versus $c$. 


\section{Capítulo 4}

\section{Modelo de infecção cruzada I}

\subsection{Descrição do modelo}

No modelo suscetível-infectado-recuperado (SIR) [5, 15, 41, 51], considerase que o processo de infecção ocorre via transmissão direta, ou seja, um indivíduo transmite a doença para outro da mesma espécie. Para investigar a propagação de uma doença cuja transmissão ocorre predominantemente por transmissão cruzada, mediada por vetores, elaborou-se um modelo de infecção cruzada com base no modelo SIR e no modelo de contato (conhecido também como processo de contato) [32, 33, 51, 53].

Considere um reticulado com estrutura espacial homogênea e isotrópica ocupado por vetores e hospedeiros. Neste modelo cada vetor pode estar em um dos seguintes estados: suscetível (S) ou infectado (I), enquanto os hospedeiros podem estar em um dos estados: suscetível (S), infectado (I) ou recuperado $(\mathrm{R})$ e permanentemente imunizado. Conforme ilustrado na figura 4.2, assumiu-se que a dinâmica de evolução é similar ao processo de contato para a população de vetores e análoga ao modelo SIR para a população de hospedeiros. Também, considerou-se que a epidemia tenha uma duração muito inferior ao tempo de vida dos hospedeiros de forma que os processos de natalidade e mortalidade possam ser desconsiderados e assim, durante o tempo de duração da epidemia, a população seja constante.

No princípio da epidemia, todos os indivíduos (vetores e hospedeiros) estão suscetíveis a determinada doença. A partir de uma pequena perturbação deste estado de equilíbrio, advinda da introdução de um único indivíduo infectado de qualquer uma das espécies envolvidas, começa a disseminação da doença. O processo de infecção é catalítico e envolve vetores e hospedeiros $\left(S_{h} I_{v} \rightarrow I_{h} I_{v}\right)$ e/ou $\left(S_{v} I_{h} \rightarrow I_{v} I_{h}\right)$, em que o subíndice $v$ e $h$ denotam vetor e hospedeiro, respectivamente. O processo de recuperação e imunização dos 
hospedeiros é espontâneo $\left(I_{h} \rightarrow R_{h}\right)$. Vetores Infectados morrem e imediatamente nasce um novo vetor suscetível em que $\left(I_{v} \rightarrow S_{v}\right)$. Este último processo significa que os vetores suscetíveis e infectados morrem espontaneamente, mas a reprodução é rápida de tal forma que o espaço seja ocupado por um novo vetor suscetível em tempo suficientemente pequeno e, neste caso, não é preciso escrever o processo de morte um vetor suscetível e o posterior nascimento de um novo vetor suscetível.

Todas as reações locais são irreversíveis e, por isso, seguindo a dinâmica de evolução, o sistema sempre atinge um estado absorvente. Se a taxa de infecção for alta, a doença se espalha, provocando uma epidemia e, portanto, o estado absorvente apresenta hospedeiros suscetíveis e recuperados (indivíduos que foram infectados e recuperaram espontaneamente) e vetores suscetíveis. Quando a taxa de infecção é baixa e o tempo de vida dos vetores é curto, a doença não se espalha e a densidade de hospedeiros recuperados é quase nula para um população grande. Por isso, neste caso o estado absorvente apresenta hospedeiros e vetores suscetíveis.

A partir da análise destas características, conclui-se que este modelo exibe uma transição de fase, pois, devido às regras de transição locais, a taxa de transição entre dois microestados gerados nos instantes $t$ e $t+\Delta t$ não obedece ao balanceamento detalhado. Pela conjectura de Grassberger [42, modelos epidêmicos com imunização pertecem à classe de universalidade da percolação dinâmica e, no ponto crítico, o estado absorvente é formado por indivíduos suscetíveis e recuperados. Os indivíduos recuperados formam um aglomerado que pode percolar a rede. Serão utilizados os métodos da mecânica estatística de não-equilíbrio para estudar esta transição de fase e obter o comportamento crítico de algumas grandezas importantes.

Devido à grande importância da estrutura espacial [36, 74] em modelos envolvendo interações de curto alcance, definiu-se o modelo em uma rede regular evoluindo com dinâmica temporal assíncrona em que cada indivíduo interage com seus primeiros vizinhos. Adotou-se a estrutura de uma rede regular quadrada como ilustrado na figura 4.1, a qual pode ser dividida em duas sub-redes A e B, ocupada por vetores e hospedeiros, respectivamente. Cada sítio da sub-rede A representa um vetor, ao qual foi associada uma variável estocástica $\eta_{i}$ que assume os valores $\eta_{i}=1$ (suscetível) ou $\eta_{i}=2$ (infectado). Na sub-rede B, cada sítio representa um hospedeiro, ao qual a variável estocástica associada pode assumir os valores $\eta_{i}=3,4$ ou 5 , conforme o hospedeiro esteja suscetível, infectado ou recuperado, respectivamente.

Todos os processos envolvendo mudança de estado são tratados como reações locais. Os processos espontâneos independem da interação entre os indivíduos. 


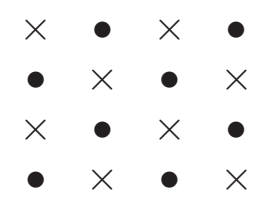

Figura 4.1: Ilustração da rede bipartida, usando $\times$ para representar um hospedeiro e $\bullet$ para denotar um vetor.
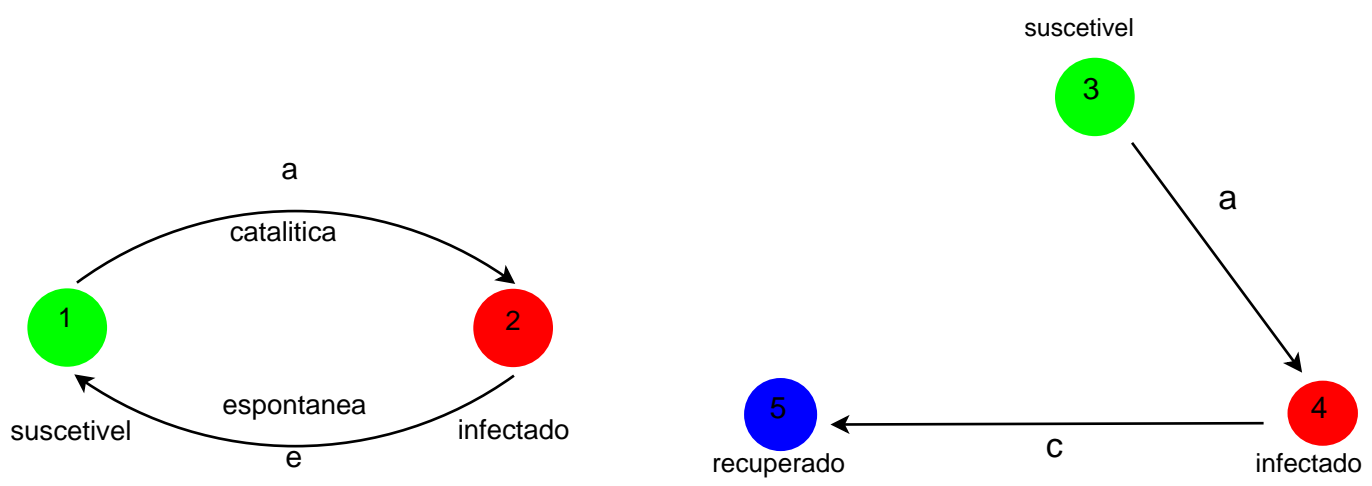

Figura 4.2: Regras de transições locais para o modelo. À esquerda, constam os estados permitidos para os vetores e à direita os estados e as transições envolvendo os hospedeiros. Somente o processo de infecção cruzada é catalítico (vetores infectados podem contaminar hospedeiros vizinhos e vice-versa) enquanto as demais transições são espontâneas.

Uma vez que o processo de evolução do modelo é um processo markoviano a tempo contínuo, pode-se reescalar o tempo e considerar a seguinte condição de normalização para as probabilidades:

$$
2 a+c+e=1
$$

em que, somando todas as probabilidades, tem-se o valor unitário.

No capítulo 3 discutimos o modelo SIR, o qual é um modelo epidêmico intraespecífico com imunização. Neste capítulo, estudou-se um modelo de infecção cruzada no qual o processo de infecção envolve duas espécies distintas. Quando um indivíduo infectado de alguma das espécies é introduzido em uma população isolada composta por indivíduos não-imunizados, pode desencadear um processo epidêmico. Uma vez infectado, o vetor pode transmitir a doença para os hospedeiros vizinhos com uma certa probabilidade "a". Supondo que os vetores morrem com probabilidade "e", a qual depende de fatores naturais e um possível controle vetorial, tem-se que um vetor pode ficar infectado por um tempo médio $t_{i} \sim 1 / e$ e, após esse 
tempo, ele morre. Para um ambiente infestado de vetores, supõe-se que o espaço e a capacidade de suporte do meio possibilitam que o espaço livre, decorrente da morte de um vetor, é imediatamente ocupado por um novo vetor. Para a população de hospedeiros, supõe-se uma dinâmica como a do modelo SIR em que um indivíduo suscetível torna-se infectado se houver pelo menos um vetor infectado em sua vizinhança. Enquanto estiver infectado, o hospedeiro pode transmitir a doença para os vetores suscetíveis que entrarem em contato com ele. Após certo tempo, o indivíduo espontaneamente se recupera, torna-se imune e deixa de transmitir a doença.

Com estas hipóteses, percebe-se que o espalhamento da doença está relacionado às probabilidades $a, e$ e $c$. Fixados valores para as probabilidade de morte $e$ e de imunização $c$, haverá um limiar para a probabilidade de infecção a necessário para a doença se espalhar. Abaixo desse limiar, a doença cessa em um curto intervalo de tempo, mas, acima desse limiar, pode se espalhar por um tempo longo, provocando uma epidemia. Como o processo de imunização dos hospedeiros infectados é um processo espontâneo, a epidemia sempre cessa para uma população finita. Devido a esses fatores, quando a epidemia acaba, tem-se um regime estacionário formado por vetores suscetíveis e hospedeiros suscetíveis e recuperados. Ao atingir esse estado, nenhuma reação pode ocorrer, visto que não há mais infectados. Consequentemente, tal qual no modelo SIR, todos os estados estacionários são estados absorventes.

As definições discutidas anteriormente visam capturar os princípios básicos associados à transmissão de doenças, entre duas espécies como a febre amarela [75], dengue [76-79], leishmaniose [80, 81], ferrugem asiática [82], cólera [83], e modelos vetor-hospedeiro [84,85], analisando explicitamente a localização espacial dos indivíduos.

Neste trabalho, será investigada de que maneira grandezas como a densidade de indivíduos suscetíveis e infectados evoluem no tempo. Também será estudado como o valor estacionário dessas grandezas varia em função da probabilidade de infecção e da probabilidade de morte dos vetores. Com o intuito de determinar as probabilidades críticas que separam as fases com espalhamento da epidemia e sem espalhamento, será investigado o comportamento crítico de algumas grandezas importantes, como o número médio de recuperados e a probabilidade da epidemia percolar em uma rede regular, e analisada a distribuição dos aglomerados formados pelos indivíduos recuperados.

O estudo deste modelo está dividido nos seguintes tópicos: na seção 4.2, será apresentado o desenvolvimento da equação mestra para o modelo. A partir da equação mestra, será aplicada a aproximação de campo médio simples (seção 4.3) e por pares (seção 4.4). Nas seções 4.5 a 4.6, serão usadas 
simulações computacionais do modelo para determinar o limiar de infecção em função da probabilidade de morte dos vetores e analisado o comportamento crítico de algumas grandezas, obtendo-se também os expoentes críticos associados a cada uma delas.

\subsection{Equação mestra para o modelo}

Como no estudo do modelo SIR (seção 3.2), será considerado um reticulado com $N$ sítios e cada configuração do sistema será representada por

$$
\eta=\left(\eta_{1}, \eta_{2}, \ldots, \eta_{i}, \ldots, \eta_{N}\right),
$$

em que $\eta_{i}$ é uma variável estocástica associada ao i-ésimo sítio e que pode assumir um dos seguintes valores: $\eta_{i}=1$ (vetor suscetível), $\eta_{i}=2$ (vetor infectado), $\eta_{i}=3$ (hospedeiro suscetível), $\eta_{i}=4$ (hospedeiro infectado) e $\eta_{i}=5$ (hospedeiro recuperado).

Para escrever as equações de evolução dos momentos da distribuição de probabilidades é conveniente escrever a probabilidade de transição por sítio como a seguir:

$$
\omega_{i}(\eta)= \begin{cases}\frac{a}{\zeta} \sum_{\delta} \delta\left(\eta_{i+\delta}, 4\right) & \text { se } \eta_{i}=1 \\ \mathrm{e} & \text { se } \eta_{i}=2 \\ \frac{a}{\zeta} \sum_{\delta} \delta\left(\eta_{i+\delta}, 2\right) & \text { se } \eta_{i}=3 \\ c & \text { se } \eta_{i}=4 \\ 0 & \text { se } \eta_{i}=5\end{cases}
$$

A partir da equação mestra (3.3), considerando a dinâmica de um único sítio a cada atualização, a evolução temporal de uma grandeza média é dada pela equação

$$
\frac{d<f(\eta)>}{d t}=\sum_{i=1}^{N}<\left[f\left({ }^{i} \eta\right)-f(\eta)\right] \omega_{i}(\eta)>
$$

em que $\eta$ é um vetor (no sentido algébrico e não-epidemiológico) que descreve o estado formado pelas $N$ partículas do aglomerado no instante $t$, sendo o estado ${ }^{i} \eta$ obtido a partir de $\eta$ pelas mudanças anticíclicas do estado do iésimo sítio (seguindo o diagrama da figura 4.2): $(2 \rightarrow 1,1 \rightarrow 2)$ e $(3 \rightarrow$ $5,4 \rightarrow 3,5 \rightarrow 4)$.

Será calculada a evolução da densidade de vetores suscetíveis e infectados, hospedeiros suscetíveis, infectados e recuperados. A densidade de vetores infectados é calculada usando $f(\eta)=\delta\left(\eta_{i}, 2\right)$ e, para facilitar a notação, será 
definido o valor médio $<\delta\left(\eta_{i}, 2\right)>=P_{i}(2)$. Como não foram consideradas explicitamente natalidade ou mortalidade, a população é constante em qualquer instante e as densidades de vetores devem seguir a condição de normalização

$$
P(1)+P(2)=1 \text {. }
$$

Portanto, pode-se calcular somente $P(1)$ ou $P(2)$. Será calculada a densidade de vetores infectados e, se for necessário calcular a densidade de vetores suscetíveis, é suficiente fazer $P(1)=1-P(2)$.

Substituindo $f(\eta)=\delta\left(\eta_{i}, 2\right)$ e a probabilidade de transição por sítio $\omega_{i}(\eta)$ na equação (4.2), obtem-se:

$$
\begin{aligned}
\frac{d P_{i}(2)}{d t}= & <\left(\delta\left({ }^{i} \eta_{i}, 2\right)-\delta\left(\eta_{i}, 2\right)\right) \omega_{i}(\eta)> \\
& =<\left(\delta\left(\eta_{i}, 1\right) \omega_{i}(\eta)-\delta\left(\eta_{i}, 2\right) \omega_{i}(\eta)>\right. \\
& =<\frac{a}{\zeta} \sum_{\delta} \delta\left(\eta_{i}, 1\right) \delta\left(\eta_{i+\delta}, 4\right)-e \delta\left(\eta_{i}, 2\right)> \\
& =a P_{i, j}(14)-e P_{i}(2) .
\end{aligned}
$$

Repetindo o procedimento para as densidades de hospedeiros suscetíveis e infectados, com a densidade de hospedeiros recuperados estabelecida via a condição de normalização,

$$
P(5)=1-P(3)-P(4) .
$$

Aplicando $f(\eta)=\delta\left(\eta_{i}, 3\right)$ na equação (4.2), tem-se:

$$
\begin{aligned}
\frac{d P_{i}(3)}{d t}= & <\left(\delta\left({ }^{i} \eta_{i}, 3\right)-\delta\left(\eta_{i}, 3\right)\right) \omega_{i}(\eta)> \\
& =<\left(\delta\left(\eta_{i}, 5\right) \omega_{i}(\eta)-\delta\left(\eta_{i}, 3\right) \omega_{i}(\eta)>\right. \\
& =<0-\frac{a}{\zeta} \sum_{\delta} \delta\left(\eta_{i}, 3\right) \delta\left(\eta_{i+\delta}, 2\right)> \\
& =-a P_{i, j}(32),
\end{aligned}
$$

e, $\operatorname{com} f(\eta)=\delta\left(\eta_{i}, 4\right)$, pode-se escrever:

$$
\begin{aligned}
\frac{d P_{i}(4)}{d t}= & <\left(\delta\left({ }^{i} \eta_{i}, 4\right)-\delta\left(\eta_{i}, 4\right)\right) \omega_{i}(\eta)> \\
& =<\left(\delta\left(\eta_{i}, 3\right) \omega_{i}(\eta)-\delta\left(\eta_{i}, 4\right) \omega_{i}(\eta)>\right. \\
& =<\frac{a}{\zeta} \sum_{\delta} \delta\left(\eta_{i}, 3\right) \delta\left(\eta_{i+\delta}, 2\right)-c \delta\left(\eta_{i}, 4\right)> \\
& =a P_{i, j}(32)-c P_{i}(4) .
\end{aligned}
$$


As densidades conjuntas na forma $P(\alpha \beta)$ representam a densidade de indivíduos $\alpha$ que tem como vizinhos indivíduos $\beta$. Por exemplo, na equação (4.7) o termo $P_{i j}(32)$ é o total de hospedeiros suscetíveis que têm vetores infectados como primeiros vizinhos. 


\subsection{Aproximação de campo médio simples}

As equações (4.4), (4.6) e (4.7) foram obtidas com o intuito de calcular as densidades $P(2), P(3)$ e $P(4)$ que envolvem correlações de um único sítio. Contudo, essas equações dependem de probabilidades conjuntas que envolvem correlações entre dois sítios vizinhos. Não foi encontrada uma solução exata para este conjunto de equações, mas é possível analisá-las por meio de aproximações de campo médio. A aproximação mais simples que pode ser feita é considerar que a densidade cruzada envolvendo dois sítios vizinhos pode ser descorrelacionada por meio da aproximação

$$
P_{i j}(\alpha \beta)=P_{i}(\alpha) P_{j}(\beta) \text {. }
$$

Usando essa aproximação simples e assumindo que há invariância translacional $\left(P_{i j}(\alpha \beta)=P_{i}(\alpha) P_{j}(\beta)=P_{i}(\beta) P_{j}(\alpha)\right)$ e definindo as densidades de vetores infectados $v=P(2)$, hospedeiros suscetíveis $x=P(3)$ e infectados $y=P(4)$, pode-se reescrever as equações (4.4), (4.6) e (4.7) como a seguir:

$$
\begin{aligned}
& \frac{d x}{d t}=-a x v \\
& \frac{d y}{d t}=a x v-c y
\end{aligned}
$$

e

$$
\frac{d v}{d t}=a y(1-v)-e v
$$

Quando a população de hospedeiros não sofreu imunização prévia, todos os indivíduos devem estar suscetíveis $(x=1)$ e $(y=0)$; a dispersão da doença pode começar a partir de uma pequena perturbação deste estado absorvente mediante a introdução de uma pequena densidade de vetores infectados ou hospedeiros infectados. O fato da disseminação começar a partir de vetores ou hospedeiros somente é significativo na fase inicial, quando surgem os primeiros contágios. A longo prazo, ambas as condições iniciais conduzem ao mesmo comportamento dinâmico e estacionário.

Escolhendo o estado inicial formado por hospedeiros suscetíveis $(x=1)$ sem nenhum hospedeiro infectado $(y=0)$ e todos os vetores suscetíveis, com exceção de uma pequena densidade de infectados que tende a zero $(v \rightarrow 0)$, dependendo das probabilidades de transição, a doença pode se dispersar ou não.

Decorrido um tempo longo $(t \rightarrow \infty)$, as densidades atingem valores estacionários e o sistema fica preso em um estado absorvente. Devido às regras de transição entre os estados, sabe-se que, no estado estacionário, 
não há hospedeiros ou vetores infectados e, portanto, essas densidades são nulas $\left(y_{\infty}=0\right)$ e $\left(v_{\infty}=0\right)$. Como parte da população de hospedeiros suscetíveis pode ter sido infectada e depois recuperada espontaneamente, no estado estacionário a densidade de hospedeiros suscetíveis deve ter certo valor $x_{\infty} \leq 1$. Estas informações sobre os estados estacionários estão contidas nas equações (4.9)-(4.11) e tornam-se evidentes quando encontrados os pontos fixos, isto é, impondo que todas as derivadas sejam nulas, obtem-se o ponto fixo $\left(x^{*}, y^{*}, v^{*}\right)=\left(x_{\infty}, 0,0\right)$.

Linearizando o sistema de equações (4.9)-(4.11) em torno desse ponto fixo e analizando sua estabilidade, em torno desse ponto fixo, calculou-se a matriz jacobiana e a equação de autovalores

$$
\operatorname{det}(J-\lambda I)=\left|\begin{array}{ccc}
-\lambda & 0 & -a x_{\infty} \\
0 & -c-\lambda & a x_{\infty} \\
0 & a & -e-\lambda
\end{array}\right|
$$

Resolvendo o determinante, obtem-se:

$$
\lambda\left[(\lambda+c)(\lambda+e)-a^{2} x_{\infty}^{2}\right]=0
$$

Os autovalores são $\lambda=0 \mathrm{e}$

$$
\lambda^{2}+(c+e) \lambda+c e-a^{2} x_{\infty}^{2}=0
$$

Para que o ponto fixo seja estável, é necessário que as duas raízes dessa equação sejam negativas. A soma das raízes é $-(c+e)$, sendo sempre negativa, pois, tanto $c$ como $e$ são probabilidades. Para as raízes serem negativas, é necessário que o produto delas seja positivo, isto é

$$
c e>a^{2} x_{\infty}^{2}
$$

Embora essa análise não permita determinar a densidade final de hospedeiros suscetíveis $x_{\infty}$, sabe-se que, se não houver epidemia, o estado absorvente possui $x_{\infty}=1$. Substituindo esse valor na desigualdade, conclui-se que o ponto fixo é estável quando

$$
c e>a^{2}
$$

$\mathrm{Ou}$

$$
a<\sqrt{c e}=a_{c} .
$$

Dessa desigualdade, conclui-se que, para um valor de $a$ abaixo do valor crítico $a_{c}$, a probabilidade infecção não provoca epidemia. Lembrando que 
foram normalizadas as probabilidades $(2 a+c+e=1)$, pode-se escrever o ponto crítico em termos de apenas uma probabilidade, como a seguir:

$$
\begin{aligned}
& a_{c}^{2}=e\left(1-e-2 a_{c}\right) \\
& a_{c}=\sqrt{e}-e .
\end{aligned}
$$

Na figura 4.3, observa-se o diagrama de fase do modelo segundo a aproximação de campo médio simples. Neste diagrama qualquer ponto do espaço de fase abaixo da linha crítica leva ao estado estacionário absorvente de hospedeiros e vetores suscetíveis $(x, v)=(1,0)$ no qual a doença não se espalha e, consequentemente, a densidade de hospedeiros recuperados é nula. Para probabilidades de infecção acima da linha crítica, tem-se dispersão da doença, levando a uma fase cujos estados absorventes possuem densidade não-nula de hospedeiros recuperados $H_{r}>0$.

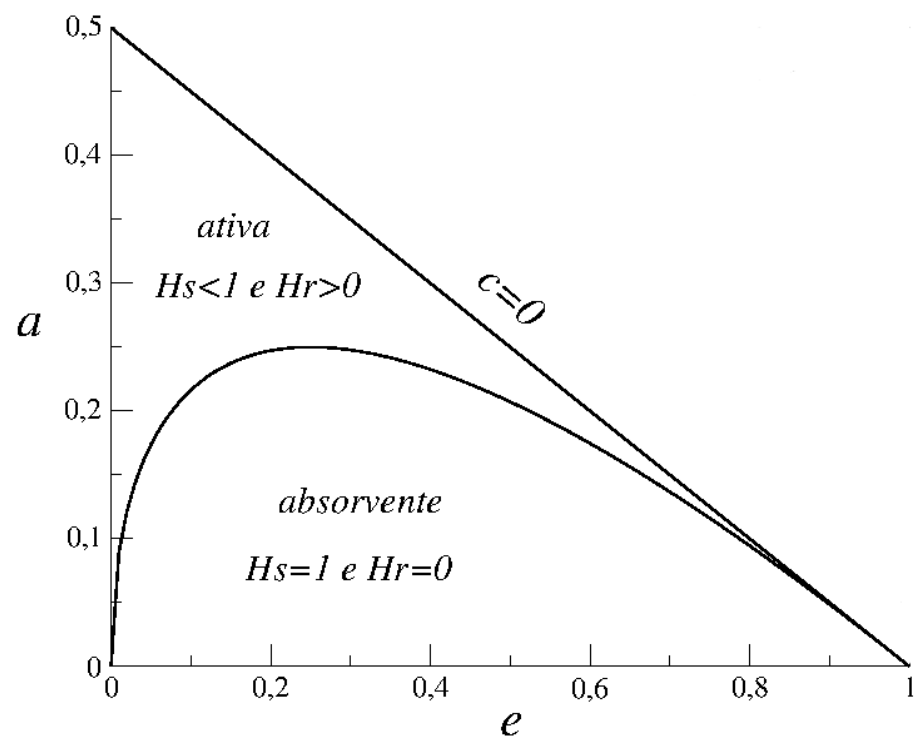

Figura 4.3: Diagrama de fase do modelo, no espaço de fase formado pelas probabilidades $e$ e $a$, segundo a aproximação de campo médio simples.

É ilustrada na figura 4.4 a evolução das densidades para as probabilidades $(e, a)=(0,05 ; 0,35)$ (gráfico à esquerda) e $(e, a)=(0,20 ; 0,35)$ (gráfico à direita). A linha contínua corresponde à densidade de hospedeiros infectados e a linha tracejada, aos vetores infectados. Se a probabilidade de morte dos vetores é muito baixa, a densidade de vetores infectados tende a ser maior que a densidade de hospedeiros infectados, o que significa que a transmissão da doença ocorre predominantemente de vetores para hospedeiros. Quando a probabilidade de morte dos vetores é grande, o tempo de vida dos vetores 
torna-se pequeno e a população de vetores infectados tende a ser menor que a de hospedeiros infectados. As curvas mostradas na figura são resultado da integração numérica das equações (4.9)-(4.11), via método de Runge-Kutta de quarta ordem, com uma densidade inicial muito baixa de vetores infectados $H_{i}(t=0)=10^{-4}$. Verifica-se também que, para ambas as probabilidades, a densidade de infectados nessa fase ativa (com espalhamento da doença) crescem até um valor máximo (não necessariamente o número máximo de infectados ocorre no mesmo instante para as duas populações). Após o atingir o valor máximo as densidades de infectados começam a decrescer, pois, devido ao processo de recuperação espontânea dos hospedeiros, o processo de infecção passa a ocorre com taxa menor que a taxa de imunização de hospedeiros e morte de vetores.
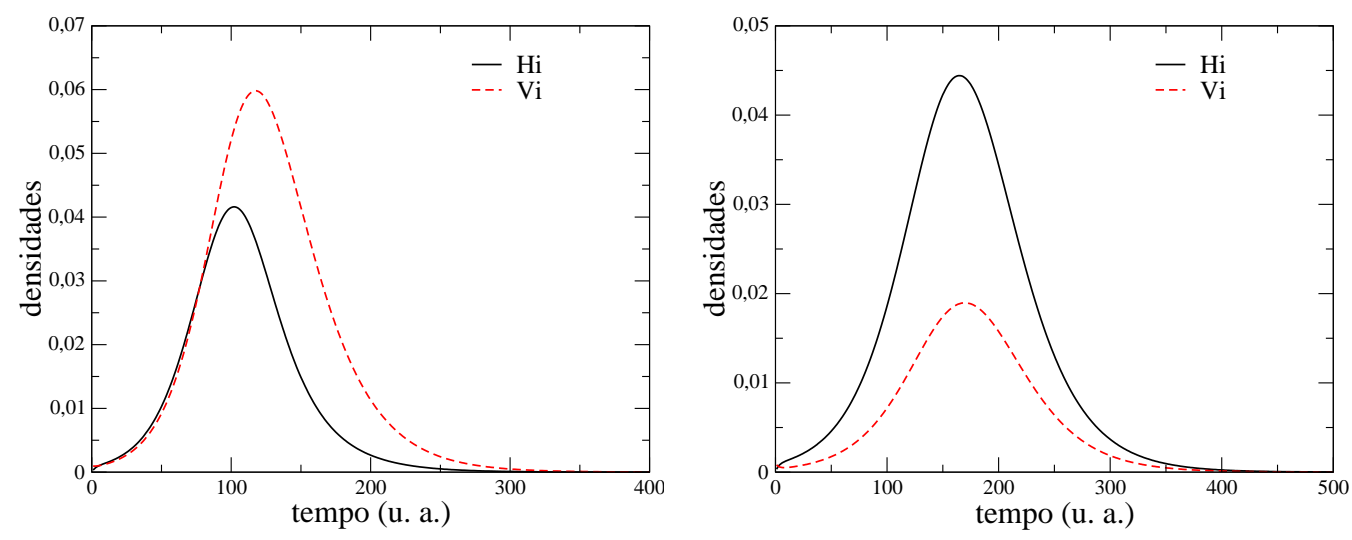

Figura 4.4: Evolução da densidade de hospedeiros (linha contínua) e vetores infectados (linha tracejada) para probabilidades $(e, a)=(0,05 ; 0,35)$ (à esquerda) e $(e, a)=(0,20 ; 0,35)$ (à direita). 


\subsection{Aproximação de campo médio por pares}

Nas equações (4.4), (4.6) e (4.7) envolvendo a evolução das densidades (primeiros momentos da distribuição de probabilidades), nota-se uma dependência de termos de segunda ordem que, na aproximação de campo médio simples, foram descorrelacionadas para limitar as correlações de apenas um sítio. É possível melhorar a aproximação calculando os termos de segunda ordem. A seguir, descreve-se o total de pares possíveis, respeitando a normalização das probabilidades:

$$
\begin{array}{r}
P(13)+P(14)+P(15)=P(1), \\
\mathbf{P}(\mathbf{2 3})+\mathbf{P}(\mathbf{2 4})+P(25)=P(2), \\
P(3)+P(4)+P(5)=1,
\end{array}
$$

e

$$
P(1)+P(2)=1 .
$$

Estão determinadas as equações para a evolução de $P(2), P(3)$ e $P(4)$. Por isso, é preciso determinar somente a evolução de dois pares e escrever todas as equações para os momentos considerados independentes. Escolhendo os pares em negrito na equação (4.19), é necessário obter a equação de evolução dos pares $P(23)$ e $P(24)$.

Usando $f(\eta)=\delta\left(\eta_{i}, 2\right) \delta\left(\eta_{j}, 3\right)$ na equação (4.2) e definindo o valor médio $<\delta\left(\eta_{i}, 2\right) \delta\left(\eta_{j}, 3\right)>=P_{i j}(23)$, obtem-se:

$$
\frac{d P_{i j}(23)}{d t}=\sum_{k=1}^{N}<\left[\delta\left({ }^{k} \eta_{i}, 2\right) \delta\left({ }^{k} \eta_{j}, 3\right)-\delta\left(\eta_{i}, 2\right) \delta\left(\eta_{j}, 3\right)\right] \omega_{k}(\eta)>.
$$

Na somatória em $k$, que percorre todos os sítios do reticulado, observa-se três casos a considerar:

- 1) Quando $k \neq i$ e $k \neq j$, tem-se: $\delta\left({ }^{k} \eta_{i}, 2\right) \delta\left({ }^{k} \eta_{j}, 3\right)-\delta\left(\eta_{i}, 2\right) \delta\left(\eta_{j}, 3\right)=\delta\left(\eta_{i}, 2\right) \delta\left(\eta_{j}, 3\right)-\delta\left(\eta_{i}, 2\right) \delta\left(\eta_{j}, 3\right)=0$.

- 2) Quando $k=i$ e $k \neq j$, tem-se: $\delta\left({ }^{i} \eta_{i}, 2\right) \delta\left({ }^{i} \eta_{j}, 3\right)-\delta\left(\eta_{i}, 2\right) \delta\left(\eta_{j}, 3\right)=\delta\left(\eta_{i}, 1\right) \delta\left(\eta_{j}, 3\right)-\delta\left(\eta_{i}, 2\right) \delta\left(\eta_{j}, 3\right)$, e o valor médio é

$$
\begin{array}{r}
<\delta\left(\eta_{i}, 1\right) \delta\left(\eta_{j}, 3\right) \omega_{i}(\eta)-\delta\left(\eta_{i}, 2\right) \delta\left(\eta_{j}, 3\right) \omega_{i}(\eta)>= \\
\frac{a}{\zeta} \sum_{\delta}<\delta\left(\eta_{i}, 1\right) \delta\left(\eta_{j}, 3\right) \delta\left(\eta_{i+\delta}, 4\right)-e \delta\left(\eta_{i}, 2\right) \delta\left(\eta_{j}, 3\right)>= \\
=a \frac{(\zeta-1)}{\zeta} P_{i+\delta i j}(413)-e P_{i j}(23) .
\end{array}
$$


- 3) Quando $k \neq i$ com $k=j$, tem-se:

$$
\begin{array}{r}
<\left[\delta\left({ }^{j} \eta_{i}, 2\right) \delta\left({ }^{j} \eta_{j}, 3\right)-\delta\left(\eta_{i}, 2\right) \delta\left(\eta_{j}, 3\right)\right] \omega_{j}(\eta)= \\
<\delta\left(\eta_{i}, 2\right) \delta\left(\eta_{j}, 5\right) \omega_{j}(\eta)-\delta\left(\eta_{i}, 2\right) \delta\left(\eta_{j}, 3\right) \omega_{j}(\eta)>= \\
<0-\frac{a}{\zeta} \sum_{\delta} \delta\left(\eta_{i}, 2\right) \delta\left(\eta_{j}, 3\right) \delta\left(\eta_{j+\delta}, 2\right)>= \\
=0-\frac{a}{\zeta} P_{i j}(23)-a \frac{(\zeta-1)}{\zeta} P_{i j j+\delta}(232) .
\end{array}
$$

Assim, a somatória em $k$ resulta a seguinte equação

$$
\frac{d P_{i j}(23)}{d t}=a \frac{\zeta-1}{\zeta}\left[P_{i+\delta i j}(413)-P_{i j j+\delta}(232)\right]-\left(e+\frac{a}{\zeta}\right) P_{i j}(23) .
$$

Para obter a equação de evolução de $P_{i j}(34)$, substituiu-se $f(\eta)=\delta\left(\eta_{i}, 3\right) \delta\left(\eta_{i}, 4\right)$ na equação (4.2) e definiu-se o valor médio $<\delta\left(\eta_{i}, 2\right) \delta\left(\eta_{i}, 4\right)>=P_{i j}(24)$. Então,

$$
\frac{d P_{i j}(24)}{d t}=\sum_{k=1}^{N}<\left[\delta\left({ }^{k} \eta_{i}, 2\right) \delta\left({ }^{k} \eta_{j}, 4\right)-\delta\left(\eta_{i}, 2\right) \delta\left(\eta_{j}, 4\right)\right] \omega_{k}(\eta)>
$$

Na somatória em $k$, deve-se analisar os três termos não-triviais:

- 1) Quando $k \neq i$ e $k \neq j$, tem-se: $\delta\left({ }^{k} \eta_{i}, 3\right) \delta\left({ }^{k} \eta_{j}, 4\right)-\delta\left(\eta_{i}, 3\right) \delta\left(\eta_{j}, 4\right)=\delta\left(\eta_{i}, 3\right) \delta\left(\eta_{j}, 4\right)-\delta\left(\eta_{i}, 3\right) \delta\left(\eta_{j}, 4\right)=0$.

- 2) Quando $k=i$ e $k \neq j$, tem-se:

$$
\begin{array}{r}
<\left[\delta\left({ }^{i} \eta_{i}, 2\right) \delta\left({ }^{i} \eta_{j}, 4\right)-\delta\left(\eta_{i}, 2\right) \delta\left(\eta_{j}, 4\right)\right] \omega_{i}(\eta)>= \\
<\delta\left(\eta_{i}, 1\right) \delta\left(\eta_{j}, 4\right) \omega_{i}(\eta)-\delta\left(\eta_{i}, 2\right) \delta\left(\eta_{j}, 4\right) \omega_{i}(\eta)>= \\
<\delta\left(\eta_{i}, 1\right) \delta\left(\eta_{j}, 4\right) \omega_{i}(\eta)-\delta\left(\eta_{i}, 2\right) \delta\left(\eta_{j}, 4\right) \omega_{i}(\eta)>= \\
\frac{a}{\zeta} \sum_{\delta}<\delta\left(\eta_{i}, 1\right) \delta\left(\eta_{j}, 4\right) \delta\left(\eta_{i+\delta}, 4\right)-e \delta\left(\eta_{i}, 2\right) \delta\left(\eta_{j}, 4\right)>= \\
=\frac{a}{\zeta} P_{i j}(14)+a \frac{(\zeta-1)}{\zeta} P_{i+\delta i j}(414)-e P_{i j}(24) .
\end{array}
$$

- 3) Quando $k \neq i$ com $k=j$, tem-se:

$$
\begin{array}{r}
<\left[\delta\left({ }^{j} \eta_{i}, 2\right) \delta\left({ }^{j} \eta_{j}, 4\right)-\delta\left(\eta_{i}, 2\right) \delta\left(\eta_{j}, 4\right)\right] \omega_{j}(\eta)= \\
<\delta\left(\eta_{i}, 2\right) \delta\left(\eta_{j}, 3\right) \omega_{j}(\eta)-\delta\left(\eta_{i}, 2\right) \delta\left(\eta_{j}, 4\right) \omega_{j}(\eta) \\
<\frac{a}{\zeta} \sum_{\delta} \delta\left(\eta_{i}, 2\right) \delta\left(\eta_{j}, 3\right) \delta\left(\eta_{j+\delta}, 2\right)-c \delta\left(\eta_{i}, 2\right) \delta\left(\eta_{j}, 4\right)>= \\
=\frac{a}{\zeta} P_{i j}(23)+a \frac{(\zeta-1)}{\zeta} P_{i j j+\delta}(232)-c P_{i j}(24) .
\end{array}
$$


Assim, a somatória em $k$ resulta a seguinte equação

$$
\begin{array}{r}
\frac{d P_{i j}(24)}{d t}=\frac{a}{\zeta}\left[P_{i j}(14)+P_{i j}(23)\right]+a \frac{\zeta-1}{\zeta}\left[P_{i+\delta i j}(414)+P_{i j j+\delta}(232)\right] \\
-(c+e) P_{i j}(24) .
\end{array}
$$

Note que há uma hierarquia de dependência de termos de ordem superior. Os momentos de primeira ordem dependem dos de segunda e os segundos dependem dos de terceira ordem. Na aproximação de campo médio por pares, as probabilidades envolvendo três sítios foram descorrelacionadas usando-se a equação

$$
P(\alpha \beta \gamma)=\frac{P(\alpha \beta) P(\beta \gamma)}{P(\beta)},
$$

em que $\alpha, \beta$ e $\gamma$ são variáveis arbitrárias. Aplicando esta aproximação e definindo as densidades $v=P(2), x=P(3), y=P(4), r=P(23)$ e $s=$ $P(24)$, é possível reescrever os trios que apareceram nas equações anteriores

$$
\begin{array}{r}
P(232)=\frac{P(23) P(32)}{P(3)}=\frac{r^{2}}{x}, \\
P(414)=\frac{P(41) P(14)}{P(1)}=\frac{[P(4)-P(24)]^{2}}{1-P(2)}=\frac{(y-s)^{2}}{1-v} .
\end{array}
$$

Nesta aproximação, as equações (4.4), (4.6), (4.7), (4.25) e (4.29) podem ser escritas como a seguir:

$$
\begin{array}{r}
\frac{d v}{d t}=a(y-s)-e v, \\
\frac{d x}{d t}=-a r, \\
\frac{d y}{d t}=a r-c y, \\
\frac{d r}{d t}=a \frac{\zeta-1}{\zeta}\left[\frac{(y-s)(x-r)}{1-v}-\frac{r^{2}}{x}\right]-\left(e+\frac{a}{\zeta}\right) r,
\end{array}
$$

e

$$
\frac{d s}{d t}=\frac{a}{\zeta}(y+r)+a \frac{\zeta-1}{\zeta}\left[\frac{(y-s)^{2}}{1-v}+\frac{r^{2}}{x}\right]-\left(\frac{a}{\zeta}+c+e\right) s .
$$

O sistema de equações (4.33)-(4.37) apresenta o ponto fixo $\left(x^{*}, y^{*}, r^{*}, s^{*}, v^{*}\right)=\left(x_{\infty}, 0,0,0,0\right)$. Na análise de estabilidade, em vez de buscar determinar os autovalores, como o interesse é saber apenas a estabilidade das soluções, pode-se analisar o limite em que os autovalores são nulos. 
Neste caso a equação de autovalores, com a matriz jacobiana calculada no ponto fixo e adotando $x_{\infty}=1$ (estado absorvente de hospedeiros suscetíveis), é dada pela seguinte expressão:

$$
-c\left(e+c+\frac{a}{\zeta}\right)\left(e+\frac{a}{\zeta}\right)+a^{2} e \frac{\zeta-1}{\zeta}+a^{2} c\left(\frac{\zeta-1}{\zeta}\right)^{2}=0,
$$

em que a probabilidade $c=1-2 a-e$.

Observa-se alguns casos particulares dessa equação:

- 1) Caso $e=0$

Neste caso, a probabilidade crítica é

$$
\begin{array}{r}
\left(1-2 a+\frac{a}{\zeta}\right)=a \frac{(\zeta-1)^{2}}{\zeta} \\
a=\frac{1}{\zeta}
\end{array}
$$

Se a rede tiver coordenação $\zeta=2$, o valor crítico é $a_{c}=1 / 2$ e o modelo não exibe transição de fase, pois a probabilidade $a$ está restrita ao intervalo $[0,1 / 2]$.

Se a rede for quadrada, isto é, o número de coordenação $\zeta=4$, o valor crítico é $a_{c}=1 / 4$ e, nesse caso, diferentemente do que ocorre no campo médio simples, a probabilidade de infecção não se anula para $e \rightarrow 0$.

- 2) Rede quadrada $(\zeta=4) \operatorname{com} e \neq 0$.

Quando isso ocorre, a equação (4.38), usando $c=1-2 a-e$, fica com termos cúbicos em $a$ e quadráticos em $e$. Após simplificar a equação para explicitar uma equação de segundo grau em termos da probabilidade de infecção, tem-se:

$$
e^{2}\left(1-\frac{7 a}{4}\right)-e \frac{15}{4}\left(a-\frac{2}{3}\right)\left(a-\frac{2}{5}\right)-\frac{a}{4}(1-2 a)(1-4 a)=0,
$$

cujas raízes são

$$
e=\frac{\mu \pm \sqrt{\mu^{2}+a\left(1-\frac{7 a}{4}\right)(1-2 a)(1-4 a)}}{2\left(1-\frac{7 a}{4}\right)},
$$

em que $\mu=\frac{15}{4}\left(a-\frac{2}{3}\right)\left(a-\frac{2}{5}\right)$.

Na figura 4.5, está descrito o diagrama de fase do modelo conforme a aproximação de campo médio simples e por pares. A linha tracejada é dada pela equação (4.40), em que as raízes devem ser calculadas em acordo com o 
domínio do espaço de fase. Neste diagrama, percebe-se que no limite $e \rightarrow 1$, as duas aproximações de campo médio apresentam mesmo comportamento. Para probabilidade de morte dos vetores muito baixa, a linha crítica encontrada via aproximação de campo médio por pares indica que o limiar de infecção é não-nulo e, portanto, esse resultado é diferente do obtido via campo médio simples. Na figura 4.6, são descritas as curvas de evolução da densidade de vetores e hospedeiros infectados, calculadas via integração numérica das equações (4.33)-(4.37), usando uma densidade inicial de infectados muito baixa.

Embora o diagrama de fase no plano $a-e$ mostre que o limiar de infecção (para $e$ pequeno) cresce em função do aumento da probabilidade de morte, deve-se lembrar que $c=1-e-2 a$ e, portanto, a probabilidade de imunização está variando também. Para probabilidades $(a, e)=(0,35 ; 0,05)$, a probabilidade de imunização é $c=0,25$, por outro lado, para $(a, e)=(0,35 ; 0,20)$, tem-se $c=0,10$. Logo, as densidades de infectados para $e=0,20$ devem ser maiores que as obtidas para $e=0,05$, uma vez que a probabilidade de imunização é menor.

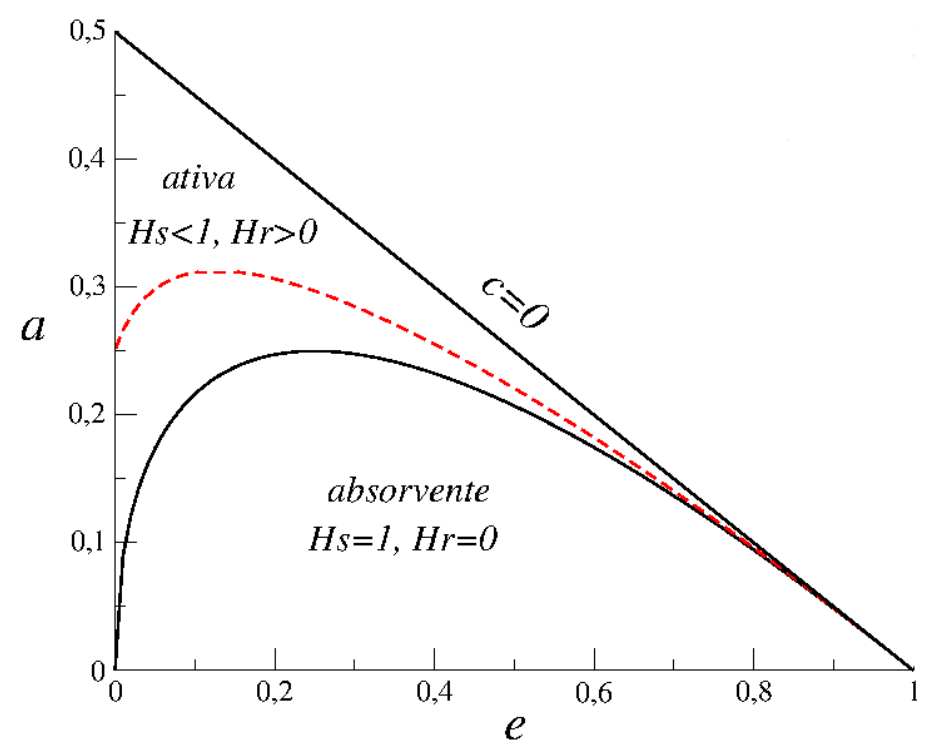

Figura 4.5: Diagrama de fase do modelo, no espaço de fase no plano $e-a$, segundo a aproximação de campo médio simples (linha contínua) e por pares (linha tracejada). 


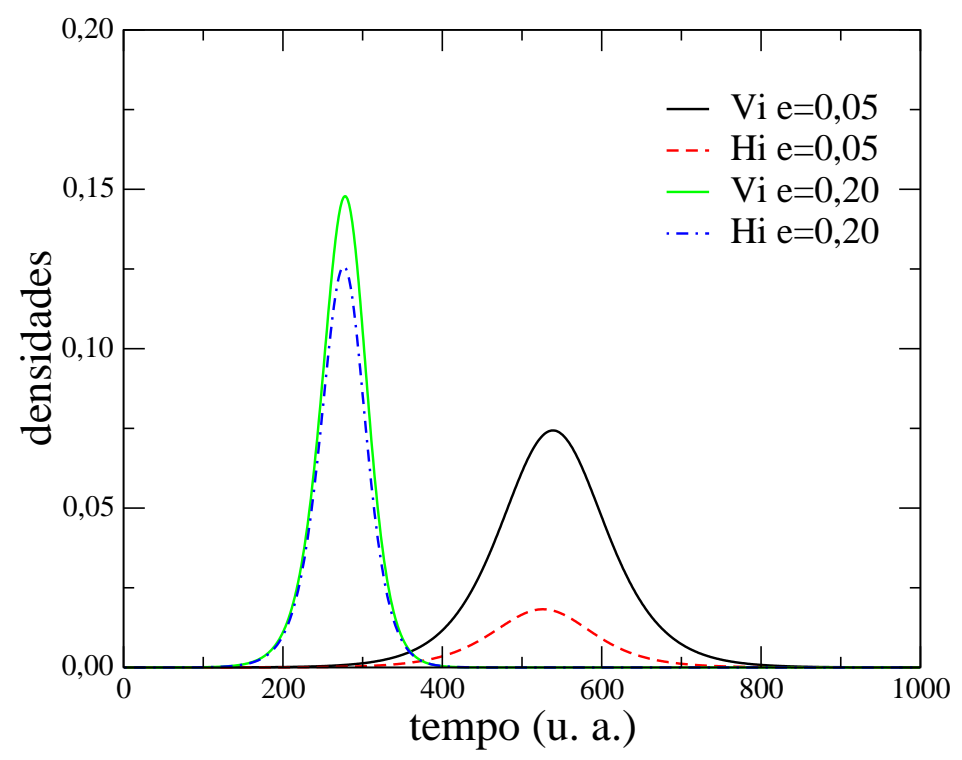

Figura 4.6: Densidade de vetores e hospedeiros infectados em função do tempo para probabilidade de infecção $a=0,35$ e diferentes probabilidades de morte dos vetores $e=0,05$ e $e=0,20$, calculadas via integração numéria das equações obtidas pela aproximação de campo médio por pares. 


\subsection{Simulaçõs computacionais estacionárias}

A partir das regras do modelo descritas na seção 4.1, elaborou-se um algoritmo para realizar as simulações do modelo definido em uma rede regular quadrada. Nesta rede, usando a vizinhança de Von Neumann, cada sítio tem quatro vizinhos $(\zeta=4)$ e, então, devido ao processo de infecção cruzada (que é catalítico), a probabilidade de um indivíduo suscetível (vetor ou hospedeiro) tornar-se infectado é $n a / 4$, em que $n$ é o número de primeiros vizinhos infectados.

Com o intuito de melhorar a velocidade de execução das simulações, é conveniente usar o método discutido na seção 3.5 com o qual se pode escrever as regras de evolução conforme os seguintes passos:

- 0) Defina uma rede quadrada com $N=2 L^{2}$ sítios e preencha alternadamente a rede com vetores e hospedeiros. Ao fazer isso, a rede pode ser analisada como uma sub-rede de vetores e outra de hospedeiros, conforme o exemplo abaixo com duas sub-redes de lado $L=2$ :

$\begin{array}{llll}1 & 3 & 1 & 3 \\ 3 & 2 & 3 & 1 \\ 1 & 3 & 1 & 3 \\ 3 & 1 & 3 & 1\end{array}$

Neste exemplo, o sítio no centro da rede $\left(\eta_{2,2}=2\right)$, destacado em vermelho, é inicialmente ocupado por um vetor infectado.

Cria-se uma lista de sítios ativos $L a$ com as coordenadas e classe do indivíduo. Como inicialmente há apenas um único indivíduo infectado, o número de vetores e hospedeiros infectados é $N_{i}=N_{2}+N_{4}=1$. A lista pode ser escrita como

$$
L a\left(n_{i}, 1\right)=L ; L a\left(n_{i}, 2\right)=L ; L a\left(n_{i}, 3\right)=2
$$

A primeira e segunda coluna deste vetor armazenam as coordenadas $i$ e $j$ do sítio, enquanto a terceira refere-se ao tipo de indivíduo (vetor ou hospedeiro infectado).

- 1) Enquanto há infectado $\left(n_{i}>0\right)$, faz-se:

- 2) Sorteia-se um sítio ativo a partir da lista de sítios ativos. Para isso, usa-se um número pseudoaleatório $\alpha \in[0,1)$ e encontra-se a posição $k$ do sítio na lista de infectados usando a função inteiro $k=i n t\left(1+\alpha \times n_{i}\right)$. As coordenadas e a espécie do indivíduo são então copiadas; a linha é $i=L a(k, 1)$; a coluna é $j=L a(k, 2)$; e a espécie é $\eta=L a(k, 3)$. 
- 3) Se $\eta=4$ sorteia-se um número $\beta \in[0,1)$ e compara-se à probabilidade de recuperação.

- 3.1) Se $\beta<c$, então o hospedeiro recupera-se rede $(i, j)=5$.

Reagrupa-se a lista de infectados

$\operatorname{La}(k,:)=\operatorname{La}\left(N_{i},:\right)$

$\operatorname{La}\left(N_{i},:\right)=0$

$N_{i}=N_{i}-1, N_{r}=N_{r}+1$.

A sintaxe adotada é tal que os dois pontos significam todas colunas da k-ésima ou n-ésima linha.

- 3.2) Senão, sorteia-se um sítio vizinho com coordenadas $(x, y)$. Se suscetível, esse vizinho torna-se infectado com probabilidade unitária. As variáveis são atualizadas fazendo-se

$N_{i}=N_{i}+1$

rede $(x, y)=2$

$\operatorname{La}\left(N_{i}, 1\right)=x ; \operatorname{La}\left(N_{i}, 2\right)=y ; \operatorname{La}\left(N_{i}, 3\right)=2$.

- 4) Se $\eta=2$ sorteia-se um número $\beta \in[0,1)$ e compara-se à probabilidade de recuperação.

- 4.1) Se $\beta<e$, então o vetor se recupera rede $(i, j)=1$.

A lista de infectados deve ser reagrupada para não ficar lacunas.

$\operatorname{La}(k,:)=\operatorname{La}\left(N_{i},:\right)$

$\operatorname{La}\left(N_{i},:\right)=0$

$N_{i}=N_{i}-1$.

- 4.2) Senão, sorteia-se um sítio vizinho com coordenadas $(x, y)$. Se for suscetível, este vizinho torna-se infectado com probabilidade unitária e as variáveis são atualizadas

$N_{i}=N_{i}+1$

rede $(x, y)=4$

$\operatorname{La}\left(N_{i}, 1\right)=x ; \operatorname{La}\left(N_{i}, 2\right)=y ; \operatorname{La}\left(N_{i}, 3\right)=4$.

- 5) Repitem-se os passos 1-4 até não haver mais infectados.

Entretanto, ao fazer a evolução usando este algoritmo, os processos são realizados com probabilidades diferentes, as quais denominamos $a^{\prime}, c^{\prime}$ e $e^{\prime}$ para diferenciar das probabilidades reais $a, c$ e $e$. 
Tabela 4.1: Comparação entre as reações locais que ocorrem no algoritmo otimizado e não-otimizado

\begin{tabular}{c|c|c}
\hline Processo & Não-otimizado & Otimizado \\
\hline Infecção de vetor & $v_{s}+h_{i} \stackrel{a}{\rightarrow} v_{i}+h_{i}$ & $v_{s}+h_{i} \stackrel{1-c^{\prime}}{\rightarrow} v_{i}+h_{i}$ \\
Infecção de hospedeiro & $v_{i}+h_{s} \stackrel{a}{\rightarrow} v_{i}+h_{i}$ & $v_{i}+h_{i} \stackrel{1-e^{\prime}}{\rightarrow} v_{i}+h_{i}$ \\
Recuperação de hospedeiro & $h_{i} \stackrel{c}{\rightarrow} h_{r}$ & $h_{i} \stackrel{c^{\prime}}{\rightarrow} h_{r}$ \\
Morte e nascimento de vetor & $v_{i} \stackrel{e}{\rightarrow} v_{s}$ & $v_{i} \stackrel{e^{\prime}}{\rightarrow} v_{s}$ \\
\hline
\end{tabular}

Ao comparar esse algoritmo otimizado a uma versão sem otimização, como mostrado na tabela 4.5, verifica-se que os dois algoritmos estão diretamente relacionados e, portanto, assume-se que há uma relação linear entre as probabilidades $a, c$ e $e$ com $a^{\prime}, c^{\prime}$ e $e^{\prime}$, respectivamente. Se a constante de proporcionalidade é $\alpha$, tem-se as seguintes relações envolvendo essas probabilidades:

$$
\begin{array}{r}
\alpha a=1-c^{\prime}, \\
\alpha a=1-e^{\prime}, \\
\alpha c=c^{\prime},
\end{array}
$$

e

$$
\alpha e=e^{\prime} .
$$

Usando a razão entre as equações (4.43) e (4.42), tem-se:

$$
\frac{c}{a}=\frac{c^{\prime}}{1-e^{\prime}} \quad \text { ou } \quad c=\frac{c^{\prime}}{1-e^{\prime}} a .
$$

E a razão entre as equações (4.44) e (4.42) resulta

$$
\frac{e}{a}=\frac{e^{\prime}}{1-e^{\prime}} \quad \text { ou } \quad e=\frac{e^{\prime}}{1-e^{\prime}} a .
$$

Substituindo a expressão para as probabilidades $c$ e $e$, dadas pelas equações (4.44) e (4.45), na condição de normalização, obtem-se a seguinte equação:

$$
2 a+\frac{c^{\prime}}{1-e^{\prime}} a+\frac{e^{\prime}}{1-e^{\prime}} a=1 .
$$

Colocando a probabilidade de infecção em evidência e depois isolando-a, obtem-se a equação para a probabilidade de infecção em termos das probabilidades do algoritmo otimizado

$$
a=\frac{1}{2+\frac{1-2 a^{\prime}}{1-e^{\prime}}} .
$$


Uma vez que a probabilidade de infecção é explicitada, pode-se calcular as probabilidades de recuperação dos hospedeiros e a probabilidade de morte dos vetores. Para isso, substitui-se a equação (4.48) nas equações (4.44) e (4.45), cujos resultados são:

$$
\begin{aligned}
& c=\frac{1-2 a^{\prime}-e^{\prime}}{2\left(1-e^{\prime}\right)+1-2 a^{\prime}}, \\
& e=\frac{e^{\prime}}{2\left(1-e^{\prime}\right)+1-2 a^{\prime}} .
\end{aligned}
$$

A metodologia de controle de doenças mediadas por vetores, para as quais não há vacinas, usualmente consiste em controlar a população de vetores, reduzindo-a o máximo possível para evitar que haja vetores que possam infectar e transmitir a doença para os hospedeiros. Desse modo, há um controle sobre a probabilidade de morte $e$, cujo aumento provoca uma redução no limiar de infecção $a_{c}$. Isto é, quanto menor a probabilidade de morte dos vetores, maior a probabilidade de infecção crítica $a_{c}$, pois, se houver menor quantidade de vetores, menores serão as chances de haver contato entre vetores infectados e hospedeiros suscetíveis. Dado um valor para a probabilidade $e$, valores abaixo de $a_{c}$ não geram epidemia, mas probabilidades de infecção maiores que $a_{c}$ provocam epidemias.

Por estas razões, nas simulações computacionais do modelo, procurou-se determinar pontos críticos no espaço de fase formado pelas probabilidades de infecção $a$ e morte dos vetores $e$. Isto é, dada uma probabilidade de morte dos vetores $e$, deve-se determinar a probabilidade crítica $a_{c}$ (com a probabilidade de recuperação $c$ estabelecida em termos de $a$ e $e$ via a condição de normalização).

Confome discutido na seção 4.1, este modelo possui irreversibilidade microscópica e apresenta vários estados absorventes. A fim de determinar um ponto crítico, inicialmente foram realizadas simulações de Monte Carlo estacionárias, com evolução assíncrona. Como no modelo SIR, cada realização (amostra) evolui conforme o algoritmo descrito no início dessa seção. Quando não há mais indivíduos infectados na rede, calculam-se as grandezas de interesse. As grandezas calculadas foram o número médio de hospedeiros recuperados,

$$
S=<N_{h r}>\text {; }
$$

a densidade de hospedeiros recuperados,

$$
\rho=\frac{<N_{h r}>}{N}
$$


em que $N=2 L^{2}$ é o total de sítios da sub-rede; o número quadrático médio de hospedeiros recuperados,

$$
M=<N_{h r}^{2}>\text {; }
$$

a razão entre o número quadrático médio de hospedeiros recuperados $(M)$ e o quadrado do número médio de hospedeiros recuperados $\left(S^{2}\right)$,

$$
U=\frac{<N_{h r}^{2}>}{<N_{h r}>^{2}}=\frac{M}{S^{2}}
$$

Também foi calculada a probabilidade $P$ do aglomerado de recuperados atingir a borda mais próxima do foco de infecção (linha $i=1$ ou coluna $j=1$ ). Estas grandezas são calculadas somente depois que o sistema fica preso em estado absorvente, ou seja, não há mais hospedeiros ou vetores infectados e, portanto, não há mais transição. Nas simulações estacionárias, ao fim da simulação, todos os vetores estarão suscetíveis, não sendo necessário fazer cálculos para a população de vetores. Optou-se por usar condições periódicas de contorno. Isso significa que a rede quadrada torna-se um toro, entretanto, a grandeza $P$ é calculada como se de fato houvesse uma fronteira.

$O$ intuito é determinar o limiar de infecção para diferentes probabilidades de morte. Para fins de controle de epidemias, esse problema é visto de outra forma: dada uma probabilidade de infecção característica da doença, deve-se controlar a população de vetores aumentando a probabilidade de morte, ou se houver vacina, imunizar a população de hospedeiros suscetíveis para impedir a disseminação da doença.

A precisão destes resultados permite apenas fazer uma estimativa acerca do ponto crítico. Resultados com maior precisão numérica podem ser obtidos a partir de simulações dependentes do tempo, os quais serão apresentados na seção 4.6. Uma vez determinado o ponto crítico, pode-se investigar o comportamento crítico das grandezas médias estacionárias ou dependentes do tempo que apresentem interesse físico.

A seguir, são apresentados os resultados estacionários obtidos para probabilidade de morte dos vetores baixa $\left(e^{\prime}=0,025\right)$, média $\left(e^{\prime}=0,100\right) \mathrm{e}$ elevada $\left(e^{\prime}=0,400\right)$.

Na figura 4.7, consta a densidade de hospedeiros recuperados para diferentes tamanhos de rede em função da probabilidade de infecção. À medida que a probabilidade de infecção aumenta, mais indivíduos infectam e, assim, cessada a epidemia, há uma densidade de hospedeiros recuperados que cresce em função do aumento da probabilidade de infecção.

N figura 4.8, consta a probabilidade $P$ versus $a^{\prime}$ para diferentes tamanhos de rede. Devido ao tamanho finito das redes, fixadas as probabilidade $a^{\prime}$ e $e^{\prime}$, 
na fase subcrítica $\left(a^{\prime}<a_{c}^{\prime}\right)$, para cada realização, há um aglomerado finito (que não percola) e, portanto, quanto menor o tamanho da sub-rede, maior a densidade estacionária de hospedeiros recuperados. À medida que $L$ cresce, essa densidade tende a um valor nulo para probabilidades de infecção abaixo do limiar de infecção $a_{c}$. Acima deste limiar, a doença pode se espalhar e a probabilidade de a epidemia percolar é não-nula. Nesta fase o tamanho dos aglomerados de hospedeiros recuperados é da ordem do tamanho da rede e, por isso, a densidade de indivíduos recuperados é não-nula. Quanto menor o tamanho da rede $L$, maior a quantidade de aglomerados que percolam. Conforme a probabilidade de infecção torna-se maior que o valor crítico, independentemente do tamanho da rede, a probabilidade de percolação dos aglomerados se aproxima do valor unitário e a rede tende a ficar praticamente preenchida por hospedeiros recuperados.

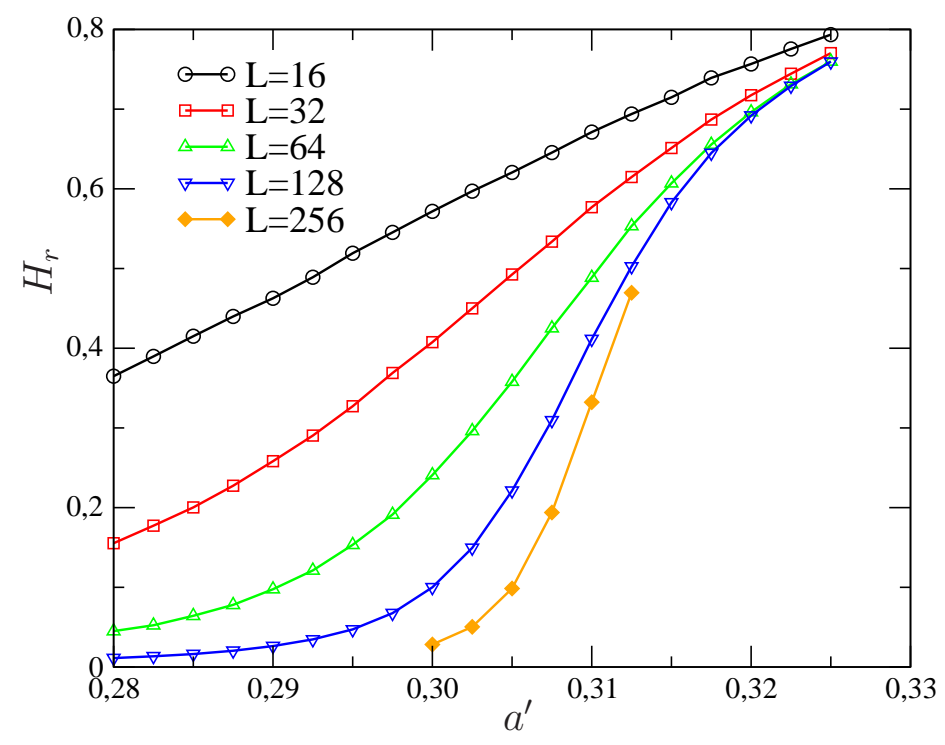

Figura 4.7: Densidade de hospedeiros recuperados $\left(H_{r}\right)$ versus a probabilidade de infecção $\left(a^{\prime}\right)$ para diferentes tamanhos de rede quadrada. A probabilidade de morte dos vetores está fixa em $\left(e^{\prime}=0,025\right)$.

O cumulante $U$ versus a probabilidade de infecção para diferentes tamanhos da rede é ilustrado na figura 4.9. Na fase subcrítica, quando $\left(a \ll a_{c}\right)$, $U$ tende a um valor constante e cresce em função do aumento da probabilidade de infecção, entretanto, quando a se aproxima do valor crítico $a_{c}, U$ decai abruptamente. Isso significa que $\left.S^{2}=<N_{h r}\right\rangle^{2}$ torna-se maior que $M=<N_{h r}^{2}>$. Na fase supercrítica, $S^{2}=<N_{h r}>^{2}$ é sempre maior que $M=<N_{h r}^{2}>$, mas, quando $\left(a \gg a_{c}\right)$, o cumulante tende ao valor unitário. 


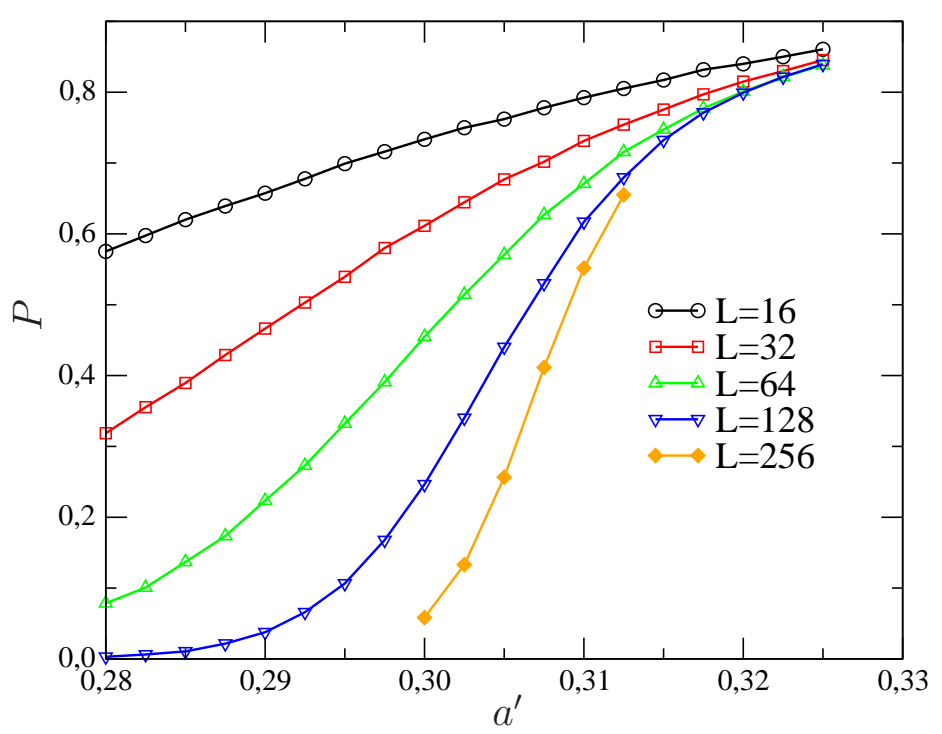

Figura 4.8: Probabilidade de a epidemia atingir a borda da rede $P$ versus a probabilidade de infecção $a^{\prime}$ para diferentes tamanhos de rede quadrada. A probabilidade de morte dos vetores está fixa em $\left(e^{\prime}=0,025\right)$.

O produto entre o cumulante $U$ e a probabilidade da epidemia atingir a borda da rede $P$ é mostrado na figura 4.10 em função da probabilidade de infecção $a^{\prime}$ para diferentes tamanhos de rede. Conforme [46] e o que foi discutido na seção 3.6, UP é universal no ponto crítico. Portanto, no limiar de infecção, as curvas de $U P$ calculadas para diferentes tamanhos de rede se cruzam. Pela figura 4.10, é possível usar este critério para estimar o limiar de infecção $a_{c}$ como sendo $a_{c}^{\prime}=0,3111(1)$ com $U P_{c}=1,0155(2)$.

Comportamento similar ao observado para as grandezas estacionárias calculadas com probabilidade de morte $e^{\prime}=0,025$ é encontrado para outras probabilidades $e^{\prime}$. A figura 4.11 exibe a densidade de hospedeiros recuperados $H_{r}$, a probabilidade $P$, o cumulante $U$ e o produto $U P$ versus $a^{\prime}$ para diferentes tamanhos de rede, com probabilidade $e^{\prime}=0,100$. Se aumentarmos ainda mais o valor de $e^{\prime}$, conforme mostrado na figura 4.12, obtemos um novo valor crítico para $a^{\prime}$. Para determinar a linha de transição de fase devemos variar $e^{\prime}$ e calcular o limiar de infecção $a^{\prime}$. Em vez de prosseguir com as simulações de Monte Carlo estacionárias, usaremos simulações de Monte Carlo dependentes do tempo para determinar os pontos críticos. 


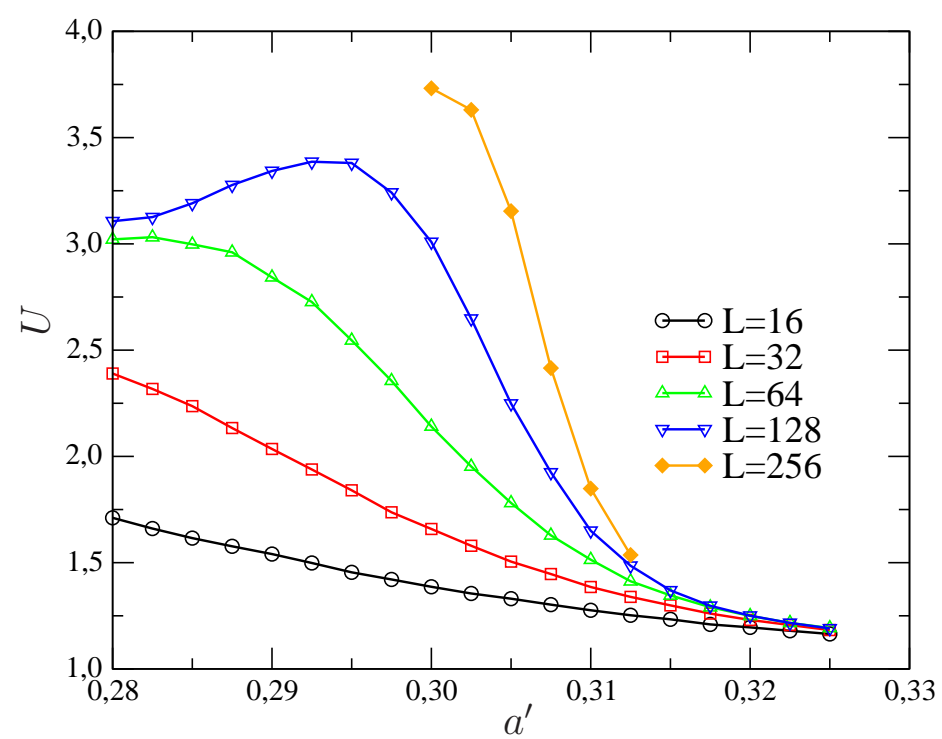

Figura 4.9: Cumulante $U=M / S^{2}$ versus a probabilidade de infecção $\left(a^{\prime}\right)$ para diferentes tamanhos de rede. A probabilidade de morte dos vetores está fixa em $\left(e^{\prime}=0,025\right)$. As probabilidades devem ser reescaladas usando-se as equações (4.48)-(4.50). 

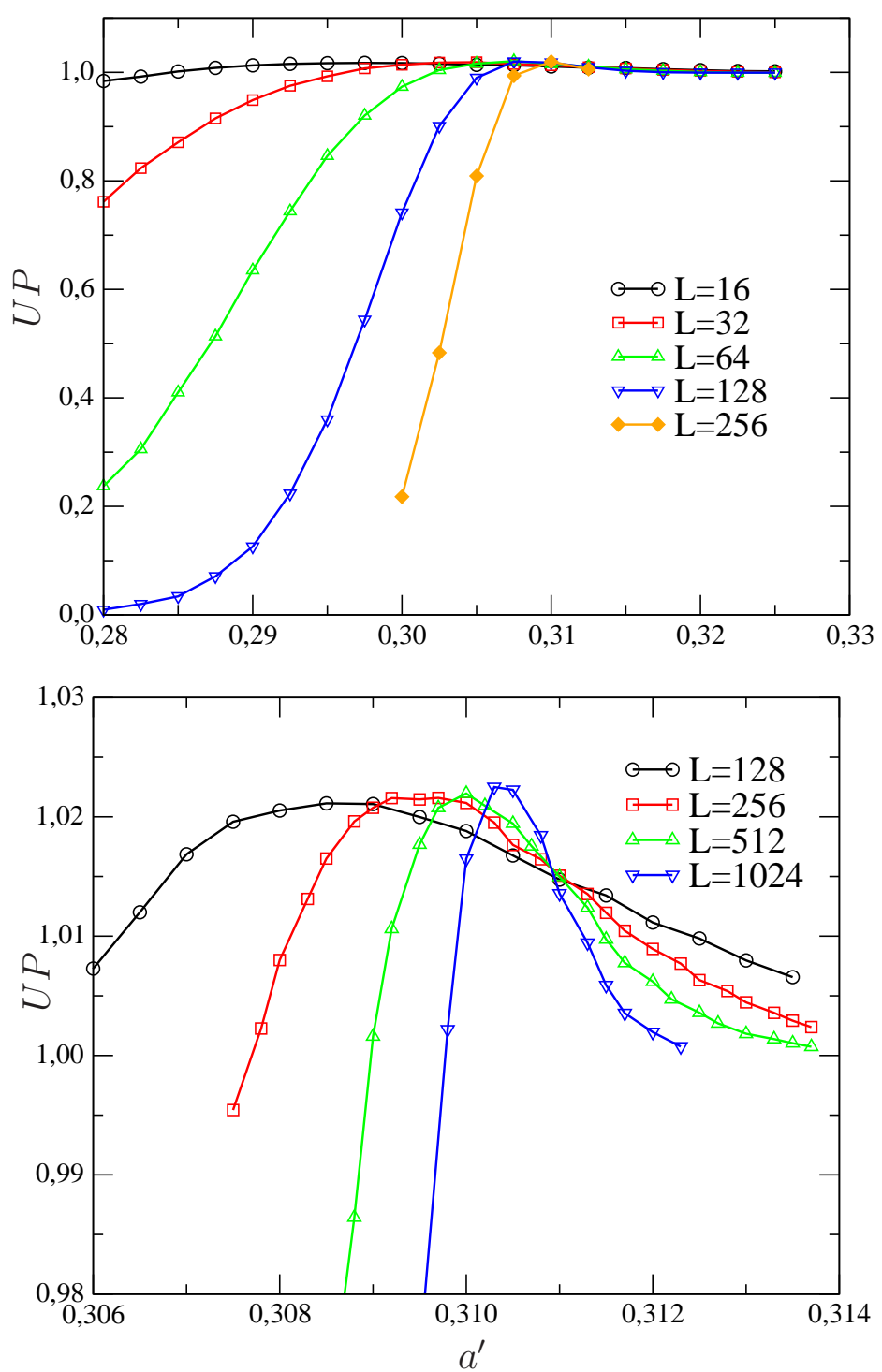

Figura 4.10: Produto do cumulante pela probabilidade de a epidemia atingir a borda da rede $U P$ versus a probabilidade de infecção $\left(a^{\prime}\right)$ para diferentes tamanhos de rede quadrada. A probabilidade de morte dos vetores está fixa em $\left(e^{\prime}=0,025\right)$. 

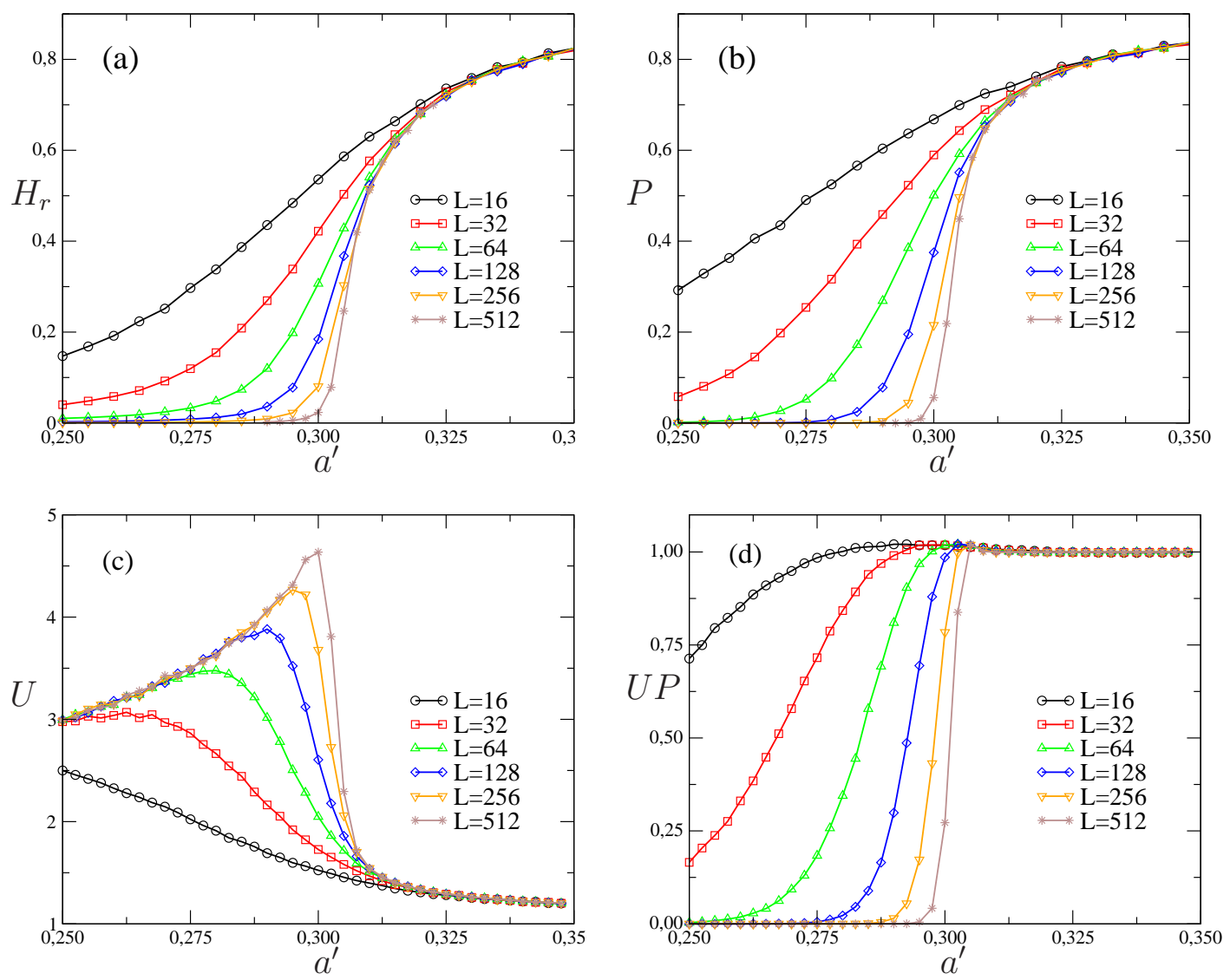

Figura 4.11: Densidade de hospedeiros recuperados $H_{r}$ (a), probabilidade de a epidemia percolar $P(\mathrm{~b})$, cumulante $U$ (b) e produto $U P(\mathrm{~d})$ em função da probabilidade de infecção $a^{\prime}$ para diferentes tamanhos de rede $L$ quando a probabilidade de morte dos vetores é $e^{\prime}=0,100$. 

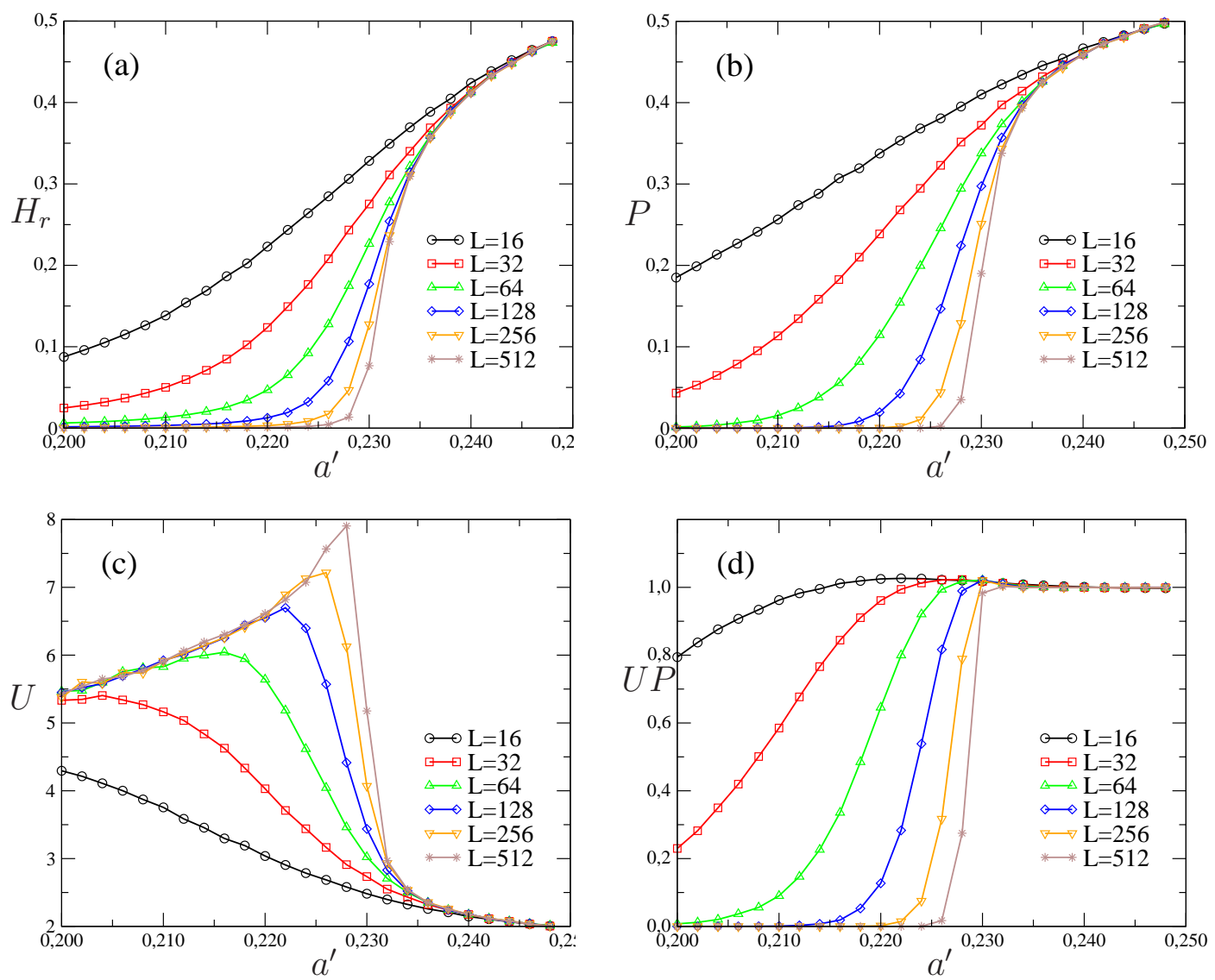

Figura 4.12: Densidade de hospedeiros recuperados $H_{r}$ (a), probabilidade de a epidemia percolar $P(\mathrm{~b})$, cumulante $U(\mathrm{c})$ e produto $U P(\mathrm{~d})$ em função da probabilidade de infecção $a^{\prime}$ para diferentes tamanhos de rede $L$ quando a probabilidade de morte dos vetores é $e^{\prime}=0,400$. 


\subsection{Simulações dependentes do tempo}

$\mathrm{Na}$ seção 4.5, foram apresentados os resultados obtidos por meio de simulações estacionárias. Nessa seção, serão apresentados resultados obtidos por meio de simulações de Monte Carlo dependentes do tempo. Usando o algoritmo com otimizações e mantendo a mesma condição inicial usada nas simulações estacionárias, tem-se inicialmente apenas um vetor infectado $\left(N_{v i}=1\right)$ e todos os hospedeiros e demais vetores suscetíveis $\left(N_{h i}=0\right)$.

Usando atualização assíncrona, a cada sorteio de um sítio ativo, o tempo é incrementado por $\Delta t=1 / N_{i}$, em que $N_{i}=N_{v i}+N_{h i}$ é o total de sítios ativos, isto é, que podem sofrer ou causar transição local. A simulação é interrompida conforme os seguintes critérios:

- (a) não haver mais sítios ativos;

- (b) a epidemia atingir uma das bordas da rede.

Se a condição $(a)$ for satisfeita, significa que o sistema está preso em um estado absorvente. A condição $(b)$ é atingida quando ocorre percolação do aglomerado de recuperados para o tamanho de rede usado na simulação.

Para este modelo, calcularam-se o número médio de indivíduos infectados $N(t)$, a probabilidade de sobrevivência da epidemia $P_{s}(t)$ (isto é, a probabilidade de haver pelo menos um indivíduo infectado) e o raio quadrático médio $R^{2}(t)$ de espalhamento dos infectados em relação ao centro da rede (foco da epidemia). Como há duas espécies diferentes, avaliou-se a evolução temporal destas grandezas médias para cada uma das espécies. Foram calculadas grandezas médias conforme as seguintes expressões:

- Número médio de infectados: para determinar o número médio de infectados da espécie $k$ ( $k=2$ ou 4 para vetores/hospedeiros infectados), é necessário percorrer toda a rede e contabilizar o total de infectados

$$
N(t)=\sum_{i, j=1}^{L} \delta\left(\eta_{i, j}, k\right),
$$

em que $\delta\left(\eta_{i, j}, k\right)$ é a função delta de Kronecker e retorna o valor um se o sítio com coordenadas $i, j$ está ocupado pela variável $k$ e nulo, caso contrário.

- Raio quadrático médio de espalhamento da epidemia: o raio quadrático médio deve ser calculado com relação ao número médio de infectados 
no instante $t$

$$
R^{2}(t)=\frac{1}{N(t)} \sum_{i=1}^{N_{a}} r^{2}(t) .
$$

Nessa soma, $r^{2}$ é a distância de cada infectado com relação ao centro da rede (foco da epidemia) e $N_{a}$ é o total de realizações.

- Probabilidade de sobrevivência: esta grandeza é a fração de realizações que estão no estado ativo no instante $t$, calculada conforme a expressão

$$
P_{s}(t)=\frac{1}{N_{a}} \sum_{i=1}^{N_{a}} H(t),
$$

em que $H(t)$ é a função degrau que assume valor unitário se houver infectado no instante $t$ ou zero, caso contrário.

Para estudar o comportamento crítico deste modelo, utilizou-se o método proposto por Grassberger e de la Torre [86] para investigar modelos com estados absorventes que exibem uma transição de fase contínua. Conforme a hipótese de escala introduzida por estes autores, as grandezas $N(t), P_{s}(t)$ e $R^{2}(t)$, calculadas para um ensemble de sistemas que evoluem a partir de um estado próximo do estado absorvente (na iminência de entrar no estado absorvente), obedecem as seguintes relações de escala:

$$
\begin{gathered}
N(t)=t^{\eta} f\left(\epsilon t^{1 / \nu_{\|}}\right), \\
P_{s}(t)=t^{-\delta} g\left(\epsilon t^{1 / \nu_{\|}}\right),
\end{gathered}
$$

e

$$
R^{2}(t)=t^{z} h\left(\epsilon t^{1 / \nu_{\|}}\right)
$$

em que $\eta, \delta$ e $z$ são os expoentes críticos associados a $N(t), P_{s}(t)$ e $R^{2}(t)$, respectivamente. As funções $f, g$ e $h$ são funções universais que dependem de $\epsilon t^{1 / \nu_{\|}}$, em que $\epsilon=a-a_{c}$ ("distância" em relação ao ponto crítico) e $\nu_{\|}$é o expoente crítico associado ao comprimento de correlação temporal. Essas relações são válidas para tempos e sistemas suficientemente grandes.

No ponto crítico $(\epsilon=0)$, as funções universais são constantes, o que torna possível reescrever as relações anteriores conforme as seguintes leis de potência:

$$
\begin{gathered}
N(t) \sim t^{\eta}, \\
P_{s}(t) \sim t^{-\delta},
\end{gathered}
$$


e

$$
R^{2}(t) \sim t^{z},
$$

em que os expoentes críticos dinâmicos $\eta, z$ e $\delta$, satisfazem a relação de hiperescala $2 \eta+4 \delta=d z$, sendo $d$ a dimensão espacial da rede.

No ponto crítico, os expoentes dinâmicos são constantes, mas, fora do ponto crítico, tornam-se funções do tempo. Para determinar o ponto crítico, é conveniente calcular estes expoentes em função do tempo e analisar seu comportamento para tempo muito grande. Para isso, calculam-se $\eta(t), \delta(t)$ e $z(t)$ em um intervalo de tempo $t-m / 2 \leqslant t \leqslant t+m / 2$, sendo $m$ o tamanho do intervalo. Neste intervalo, os expoentes são calculados usando as seguintes expressões:

$$
\begin{aligned}
& \eta(t)=\frac{\sum_{i} \log \left(\frac{i}{t}\right) \log \left(\frac{N(i)}{N}\right)}{\sum_{i}\left[\log \left(\frac{i}{t}\right)^{2}\right]}, \\
& \delta(t)=\frac{\sum_{i} \log \left(\frac{i}{t}\right) \log \left(\frac{P_{s}(i)}{P_{s}}\right)}{\sum_{i}\left[\log \left(\frac{i}{t}\right)^{2}\right]},
\end{aligned}
$$

e

$$
z(t)=\frac{\sum_{i} \log \left(\frac{i}{t}\right) \log \left(\frac{R^{2}(i)}{R^{2}}\right)}{\sum_{i}\left[\log \left(\frac{i}{t}\right)^{2}\right]},
$$

em que o índice $i$ varia entre $t-m / 2$ até $t+m / 2$, e $\bar{N}, \bar{R}^{2}$ e $\bar{P}_{s}$ são os valores médios de $N, R^{2}$ e $P_{s}$ neste intervalo.

No regime subcrítico $\left(a<a_{c}\right)$, decorrido um tempo longo, o número de infectados tende a zero e, portanto, o cálculo dos expoentes dinâmicos nessa fase mostrará que os expoentes dinâmicos não são constantes no tempo e que as grandezas $N(t), R^{2}(t)$ e $P_{s}(t)$ (relações (4.61)-(4.63)) crescem abaixo da lei de potência. Acima do ponto crítico $\left(a>a_{c}\right)$, as grandezas $P_{s}(t), N(t)$, e $R^{2}(t)$ crescem mais rápido que uma lei de potência. 

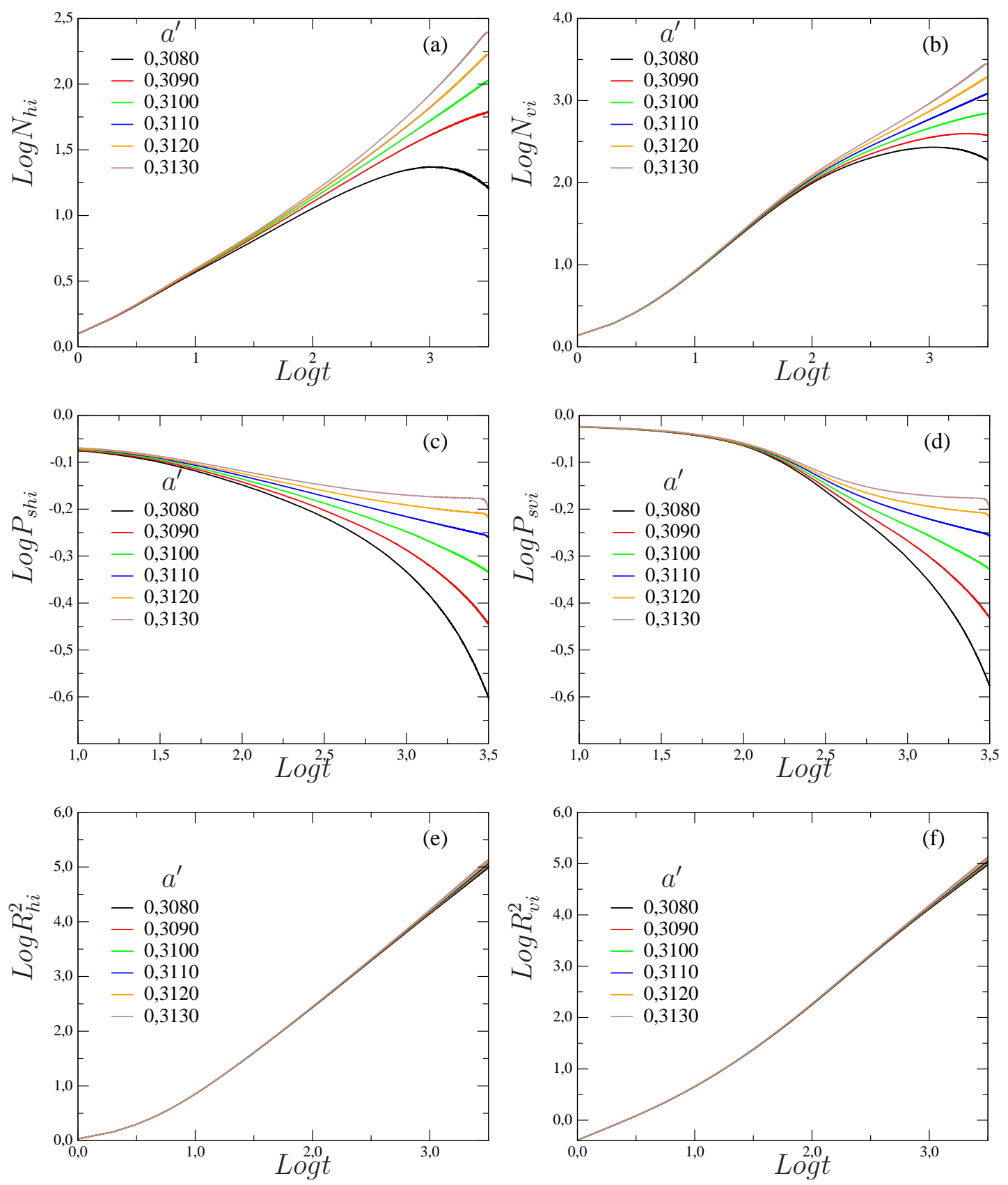

Figura 4.13: Em escala log-log: número de hospedeiros (a) e vetores (b) infectados, probabilidade de sobrevivência da epidema para hospedeiros (c) e vetores (d), raio quadrático médio de dispersão da epidemia para hospedeiros (e) vetores (f) versus tempo para probabilidade de morte dos vetores $e^{\prime}=0,025$. 
Na figura 4.13, descreve-se o comportamento das grandezas $N(t), R^{2}(t)$ e $P_{s}(t)$ versus $t$, em escala log-log, para diferentes probabilidades de infecção $a^{\prime}$ calculadas para vetores e hospedeiros infectados. As simulações foram efetuadas usando redes quadradas com $L=1024 \mathrm{com}$ um total de $5 \times 10^{6}$ realizações independentes e probabilidade de morte dos vetores $e^{\prime}=0,025$. Observando a curva de $N(t)$, tanto para hospedeiros como vetores infectados, vê-se que, na escala logarítmica, as curvas com probabilidade de infecção abaixo do ponto crítico $\left(a<a_{c}\right)$ são voltadas para baixo e, acima do ponto crítico, são convexas. Esse mesmo comportamento ocorre também para a probabilidade de sobrevivência $P_{s}(t)$ e raio quadrático médio $R^{2}(t)$.

Usando as relações (4.64) - (4.66), pode-se calcular a inclinação local de cada curva e determinar o ponto crítico e os expoentes dinâmicos. Na figura 4.14, é demonstrada a inclinação local das curvas mostradas na figura 4.13 usando séries com $m=50$ pontos. O expoente dinâmico $\eta$ associado ao número médio de infectados é o que tem melhor distinção de comportamento das fases subcrítica e supercrítica. Quando $t$ torna-se grande $(1 / t$ tendendo a zero) na fase subcrítica, $\eta(t)$ decai. Pela figura 4.14-(a), no ponto crítico, $\eta(t)$ é constante para todo $t$ e, para a fase supercrítica $\eta(t)$, é crescente em função do aumento do tempo. Esse mesmo comportamento é observado para o expoente $\delta$ associado à probabilidade de sobrevivência. O expoente dinâmico $z$ associado ao raio quadrático médio (para o total de realizações usado) apresenta muitas flutuações e, somente ajustando uma reta aos dados de $z(t)$, é possível distinguir as duas fases.

Ajustando uma reta aos dados de $P_{s}(t), N(t)$ e $R^{2}(t)$, obtêm-se os expoentes críticos. Com base nas inclinações dos exponentes, mostrados na figura 4.14. conclui-se que o limiar de infecção correspondente à probabilidade de morte $e^{\prime}=0,025$ é $a_{c}^{\prime}=0,3108(1)$ e os expoentes críticos são: $\eta=0,56(3), \delta=0,094(5)$ e $z=1,7(1)$. As incertezas advêm de dois fatores: incerteza de determinar o ponto crítico com exatidão e flutuação dos valores médios. Apesar destas limitações, o método permite obter resultados mais precisos, desde que se use tamanho de rede ainda maior (o que permite ver com maior resolução a separação das curvas de $\eta(t), \delta(t)$ e $z(t)$ próximo ao ponto crítico para tempos longos) e/ou aumentar o número de realizações (reduzir as flutuações em cada uma das curvas). 

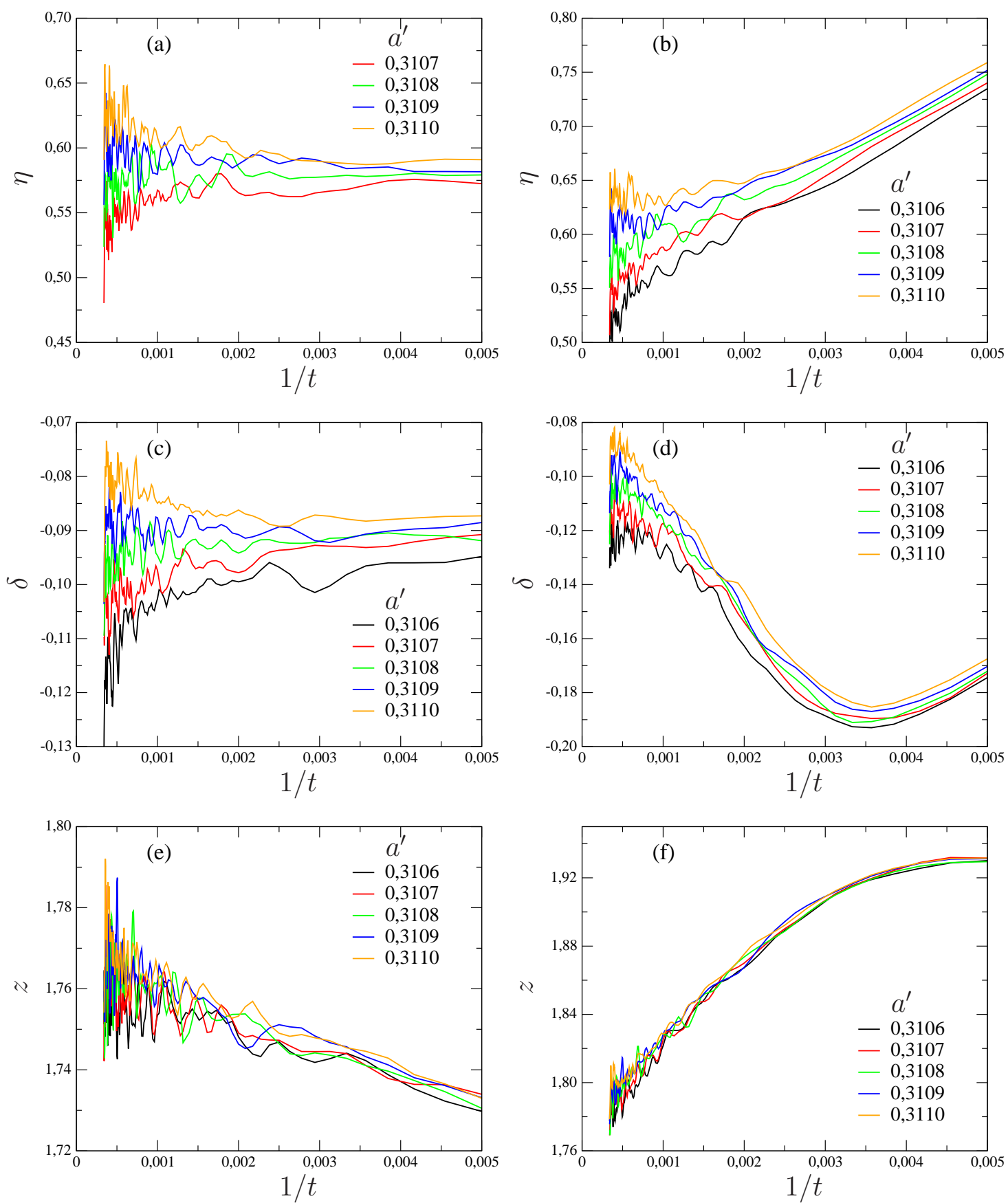

Figura 4.14: Expoentes dinâmicos em função do inverso do tempo para probabilidade de morte dos vetores $e^{\prime}=0,025$. Expoente dinâmico $\eta$ associado ao número de hospedeiros (a) e vetores (b) infectados. Expoente dinâmico $\delta$ associado à probabilidade de sobrevivência da epidemia na população de hospedeiros (c) e vetores (d). Expoente dinâmico $z$ associado ao raio quadrático médio de espalhamento da epidemia na sub-rede de hospedeiros (e) e vetores (f). 
Ambos os fatores exigem muito mais tempo computacional, mas, de todo modo, a precisão e rapidez em determinar o ponto crítico usando simulações dependentes do tempo são melhores que aquelas usando simulações estacionárias, pois, conforme mostrado na figura 4.10, a quantidade $U P$ exige que sejam feitas simulações com diferentes tamanhos de rede para diferentes probabilidades próximas ao ponto crítico. Além disso, o cumulante apresenta muitas flutuações, cuja amplitude cresce em função do aumento do tamanho da rede. Nota-se que, nas simulações dependentes do tempo, é suficiente fazer simulações com um único tamanho relativamente grande para diferentes probabilidades de infecção, tal que possibilite o ponto crítico com a precisão numérica desejada.

Conforme discutido na seção 3.8 (modelo SIR na rede triangular) e nas refêrencias 44, 71, um método muito eficaz para determinar o ponto crítico a partir das simulações estacionárias em que o o estado estacionário é sempre um estado absorvente é analisar as distribuições estacionárias dos aglomerados de hospedeiros recuperados para determinar o ponto crítico com precisão superior à encontrada via simulações dependentes do tempo. Contudo, para se obter um histograma com poucas flutuações, é necessário um conjunto muito grande de aglomerados.

Pelas figuras 4.13 e 4.14, nota-se que há uma diferença entre a evolução temporal dos expoentes dinâmicos para a população de hospedeiros e vetores. Conforme os resultados apresentados na figura 4.14 e 4.15, verifica-se que os expoentes dinâmicos apresentam diferenças de comportamento entre a população de vetores e hospedeiros. O motivo dessa diferença está ligado às probabilidades de reação local. Como vetores e hospedeiros tornam-se infectados com probabilidade $\frac{n a}{\zeta}$ mas os vetores morrem com probabilidade $e$ (e imediatamente nasce um novo vetor suscetível), enquanto os hospedeiros infectados recuperam espontaneamente com probabilidade $c$, o número médio de infectados de cada população não apresenta o mesmo valor em um dado instante de tempo. Logo, o número de primeiros vizinhos infectados $n$, em média, não é o mesmo valor para vetores e hospedeiros. O balanço entre criação de novos infectados e a aniquilação de infectados, em geral apresenta valores distintos para cada uma das populações. Consequentemente, os cálculos dos expoentes dinâmicos em função do tempo, que extraem essas informações, são afetados. Por exemplo, pela figura 4.14t(a) e 4.14t(b), ve-se que, para tempo curto (1/t grande), o expoente dinâmico $\eta$ associado ao número de hospedeiros infectados é praticamente constante no tempo para todas as probabilidades de infecção analisadas. Entretanto, o número de vetores infectados cresce com uma lei de potência cujo expoente decai em função do aumento do tempo (figura 4.13. 
(b)). Contudo, no limite de tempo longo ( $1 / t$ tende a zero), no ponto crítico, todos os expoentes críticos devem atingir um valor bem definido, tornando-se independentes do tempo.

Para determinar outros pontos críticos, é necessário repetir o procedimento para outras probabilidades de morte do vetor. Como o método é o mesmo, suprimem-se os dados e mostra-se somente a síntese dos resultados obtidos. Por exemplo, na figura 4.15, a inclinação local das curvas de $N(t), P_{s}(t)$ e $R^{2}(t)$ para hospedeiros e vetores, com probabilidade de morte $e^{\prime}=0,100$. Comparando as figuras 4.14-(d) e 4.15-(d) e 4.14-(f) e 4.15-(f), nota-se que o aumento da probabilidade de morte reduz o transiente inicial. Os expoentes dinâmicos associados determinada quantidade, tanto para hospedeiros como vetores, tendem ao mesmo valor para tempos longos. Para $e^{\prime}=0,100$, o ponto crítico é $a_{c}^{\prime}=0,30550(3)$ e os expoentes críticos dinâmi$\cos$ são $\eta=0,581(5), \delta=0,094(2)$ e $z=1,75(3)$, cujas incertezas levam em consideração as duas populações. Na tabela 4.2, constam os expoentes dinâmicos calculados para probabilidades próximas ao valor crítico para as duas populações, assim como em $e^{\prime}=0,025$ o expoente dinâmico $z$ calculado para os vetores, para cada probabilidade de infecção $a^{\prime}$, está sempre acima do valor obtido para a população de hospedeiros.

Tabela 4.2: Expoentes dinâmicos calculados para a população de vetores e hospedeiros quando a probabilidade de morte dos vetores é $e^{\prime}=0,100$

\begin{tabular}{c|c|c|c|c|c|c}
\hline & \multicolumn{3}{|c|}{ Vetores } & \multicolumn{3}{c}{ Hospedeiros } \\
\hline$a^{\prime}$ & $\eta$ & $\delta$ & $z$ & $\eta$ & $\delta$ & $z$ \\
\hline 0,30540 & 0,571 & 0,104 & 1,783 & 0,562 & 0,099 & 1,757 \\
0,30545 & 0,581 & 0,101 & 1,784 & 0,572 & 0,096 & 1,758 \\
0,30550 & 0,590 & 0,098 & 1,785 & 0,581 & 0,094 & 1,759 \\
0,30555 & 0,599 & 0,095 & 1,786 & 0,589 & 0,091 & 1,761 \\
0,30560 & 0,608 & 0,093 & 1,787 & 0,598 & 0,089 & 1,762 \\
\hline
\end{tabular}

Os expoentes dinâmicos $\eta, \delta$ e $z$ obtidos para este modelo, dentro da margem de incerteza, estão de acordo com a lei de hiperescala $(\eta+2 \delta=d z / 2)$ e concordam com os expoentes da classe de universalidade da percolação dinâmica. Isso condiz com a conjectura de Grassberger e, embora as regras de transição para a população de vetores sejam análogas à do modelo de contato, o qual pertence a classe de universalidade da percolação direcionada, a dinâmica da doença na população de hospedeiros segue as regras do modelo SIR que pertence à classe de universalidade da percolação dinâmica [87. Devido ao processo de infecção cruzada, as reações que ocorrem entre vetores ficam acopladas às reações do modelo SIR que ocorrem para a população 
de hospedeiro e, assim, prevalece o comportamento crítico do modelo SIR [34, 46, $, 51,88$.

Lembrando que as probabilidades são reduzidas (usadas no algoritmo com otimizações), todos os pontos críticos devem ser corrigidos usando as equações (4.48)-(4.50) que transformam as probabilidades reduzidas para as probabilidades de morte e infecção corretas. Na tabela 4.3, consta a síntese dos resultados obtidos. Os pontos críticos foram determinados usando em média $5 \times 10^{6}$ realizações distintas, e os expoentes críticos estão com incerteza compatível tanto com os dados da população de vetores quanto de hospedeiros.

Tabela 4.3: Síntese dos pontos críticos e expoentes críticos obtidos a partir das simulações dependentes do tempo usando o algoritmo com otimizações. A incerteza nas probabilidades e e $a$ devem ser calculadas usando propagação de erro, mas por simplicidade, ao fazer o diagrama de fase assumimos que a incerteza de $e$ e $a$ é a incerteza de $a^{\prime}$

\begin{tabular}{c|c|c|c|c|c|c}
\hline \multicolumn{3}{c|}{ Probabilidades } & \multicolumn{3}{c}{ Expoentes dinâmicos } \\
\hline \multicolumn{2}{c}{ Reduzidas } & \multicolumn{2}{c|}{ Reescaladas } & \multicolumn{2}{c}{ Expla } \\
\hline$e^{\prime}$ & $a^{\prime}$ & $e$ & $a$ & $\eta$ & $\delta$ & $z$ \\
\hline 0 & $0,31050(13)$ & 0 & 0,38310 & $0,57(2)$ & $0,09(1)$ & $1,75(2)$ \\
0,025 & $0,3108(1)$ & 0,0100 & 0,38877 & $0,56(3)$ & $0,094(5)$ & $1,7(1)$ \\
0,050 & $0,3100(1)$ & 0,02068 & 0,39293 & $0,58(2)$ & $0,093(3)$ & $1,76(1)$ \\
0,100 & $0,30550(3)$ & 0,04414 & 0,39274 & $0,581(5)$ & $0,094(2)$ & $1,75(3)$ \\
0,150 & $0,29785(1)$ & 0,07010 & 0,39722 & $0,585(3)$ & $0,093(1)$ & $1,76(1)$ \\
0,200 & $0,28770(2)$ & 0,09844 & 0,39375 & - & - & - \\
0,250 & $0,27560(2)$ & 0,12913 & 0,38738 & - & - & - \\
0,300 & $0,2619(1)$ & 0,16219 & 0,37844 & - & - & - \\
0,400 & $0,2310(2)$ & 0,23584 & 0,35376 & - & - & - \\
0,500 & $0,1968(2)$ & 0,32051 & 0,32061 & - & - & - \\
0,700 & $0,1222(3)$ & 0,53124 & 0,22768 & - & - & - \\
\hline
\end{tabular}



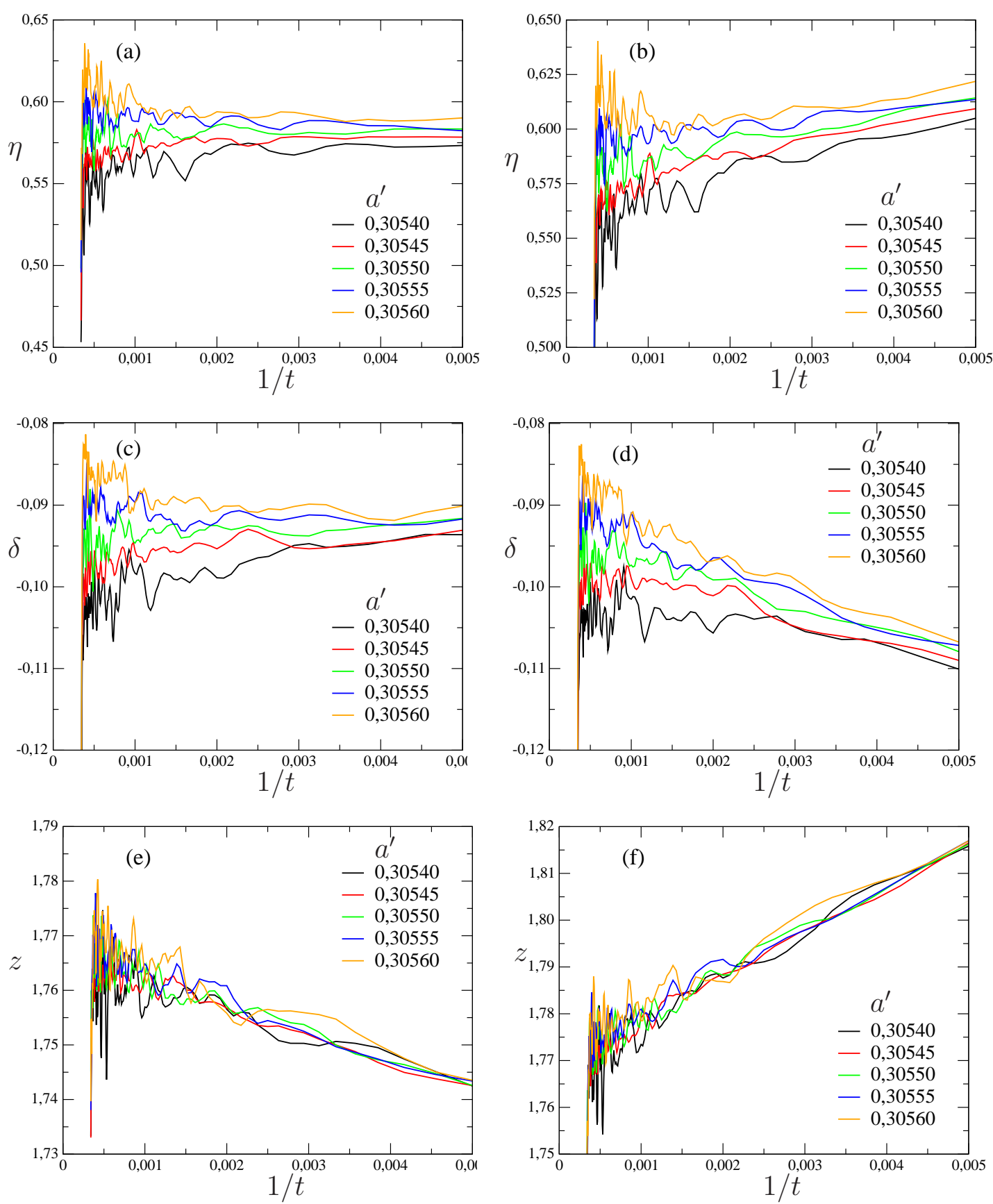

Figura 4.15: Expoentes dinâmicos em função do inverso do tempo para probabilidade de morte dos vetores $e^{\prime}=0,100$. Expoente dinâmico $\eta$ associado ao número de hospedeiros (a) e vetores (b) infectados. Expoente dinâmico $\delta$ associado à probabilidade de sobrevivência da epidemia na população de hospedeiros (c) e vetores (d). Expoente dinâmico $z$ associado ao raio quadrático médio de espalhamento da epidemia na sub-rede de hospedeiros (e) e vetores (f). 


\subsubsection{Caso $e=0$}

Quando $e=0$, não há morte dos vetores e, uma vez infectado, eles permanecem infectados indefinidamente. Neste caso, a probabilidade de sobrevivência da epidemia entre vetores é sempre unitária e forma-se um aglomerado de vetores infectados cuja evolução (e tamanho) é similar à formada pelos hospedeiros recuperados.

Na figura 4.16, estão descritos o número de infectados $N(t)$ e o raio quadrático médio $R^{2}(t)$ para hospedeiros e vetores infectados. Na figura 4.17 tem-se os expoentes $\eta, \delta$ e $z$ associados ao número médio de hospedeiros e vetores infectados, à probabilidade de sobrevivência da epidemia entre hospedeiros e ao raio de espalhamento da epidemia, respectivamente. Além disso, para mostrar que de fato a evolução do número de hospedeiros recuperados é similar ao crescimento do número de vetores infectados, calculou-se o expoente dinâmico, denominado $\eta_{h r}$, associado ao número hospedeiros recuperados. Pelo comportamento dos expoentes dinâmicos, visto na figura 4.17 pode-se inferir que o ponto crítico é $a_{c}=0,31050(3)$ e os expoentes críticos dinâmicos para a população de hospedeiros são: $\eta=0,57(2)$, $\delta=0,09(1)$ e $z=1,75(2)$, que recaem na classe de universalidade da percolação dinâmica. Contudo, os expoentes calculados para a população de vetores são: $\eta=1,57(2), z=1,75(2)$ e $\eta_{h r}=1,57(2)$. E como a probabilidade de sobrevivência é constante no tempo, não faz sentido calcular o expoente $\delta$. Percebe-se que o expoente $z$ para as duas populações assume o mesmo valor e que $\eta_{h r}=\eta \operatorname{com} \eta$ calculado para os vetores, mas esse valor é $1+0,57$ (é um expoente não universal) em que 0,57 é o expoente crítico associado ao número de hospedeiros infectados. Na tabela 4.4, constam os expoentes dinâmicos calculados para pontos próximos ao ponto crítico, os quais mostram a dispersão dos valores em relação ao valor crítico. No cálculo desses expoentes desprezamos os dados referentes ao transiente que ocorre no intervalo $t<200$ todos esses valores apresentam incerteza no último algarismo significativo com uma unidade para mais ou menos.

Verificou-se também que, conforme os resultados obtidos via aproximação de campo médio por pares, mesmo que haja vetores infectados em qualquer instante, é necessário um limiar de infecção não-nulo para que haja dispersão da doença. Além disso, mesmo na fase ativa, com espalhamento da doença, o processo de imunização acaba formando um aglomerado de hospedeiros recuperados que isola os hospedeiros suscetíveis dos vetores infectados que impede o processo de dispersão da doença indefinidamente. 

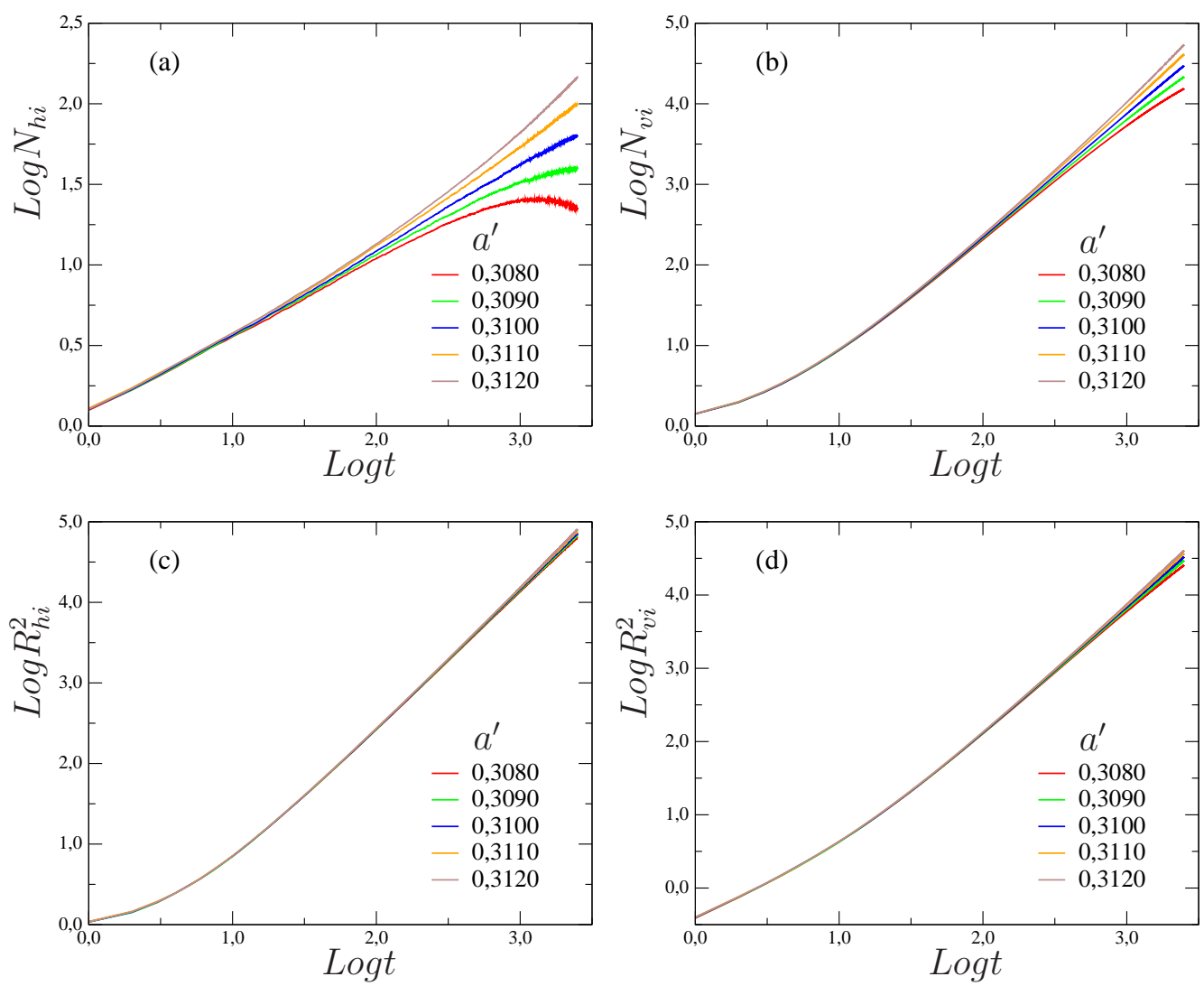

Figura 4.16: Em escala Log-Log: número médio de hospedeiros (a) e vetores infectados (b), raio quadrático médio para hospedeiros (c) e vetores (d) infectados em função do tempo para probabilidade de morte $e^{\prime}=e=0$.

Tabela 4.4: Expoentes dinâmicos calculados para a população de vetores e hospedeiros quando a probabilidade de morte dos vetores é $e^{\prime}=e=0$

\begin{tabular}{c|c|c|c|c|c|c}
\hline & \multicolumn{2}{|c|}{ Vetores } & \multicolumn{4}{c}{ Hospedeiros } \\
\hline$a^{\prime}$ & $\eta_{v i}$ & $z$ & $\eta$ & $\delta$ & $z$ & $\eta_{h r}$ \\
\hline 0,31025 & 1,543 & 1,737 & 0,534 & 0,105 & 1,750 & 1,546 \\
0,31050 & 1,572 & 1,746 & 0,576 & 0,094 & 1,753 & 1,574 \\
0,31175 & 1,595 & 1,754 & 0,609 & 0,084 & 1,758 & 1,597 \\
\hline
\end{tabular}



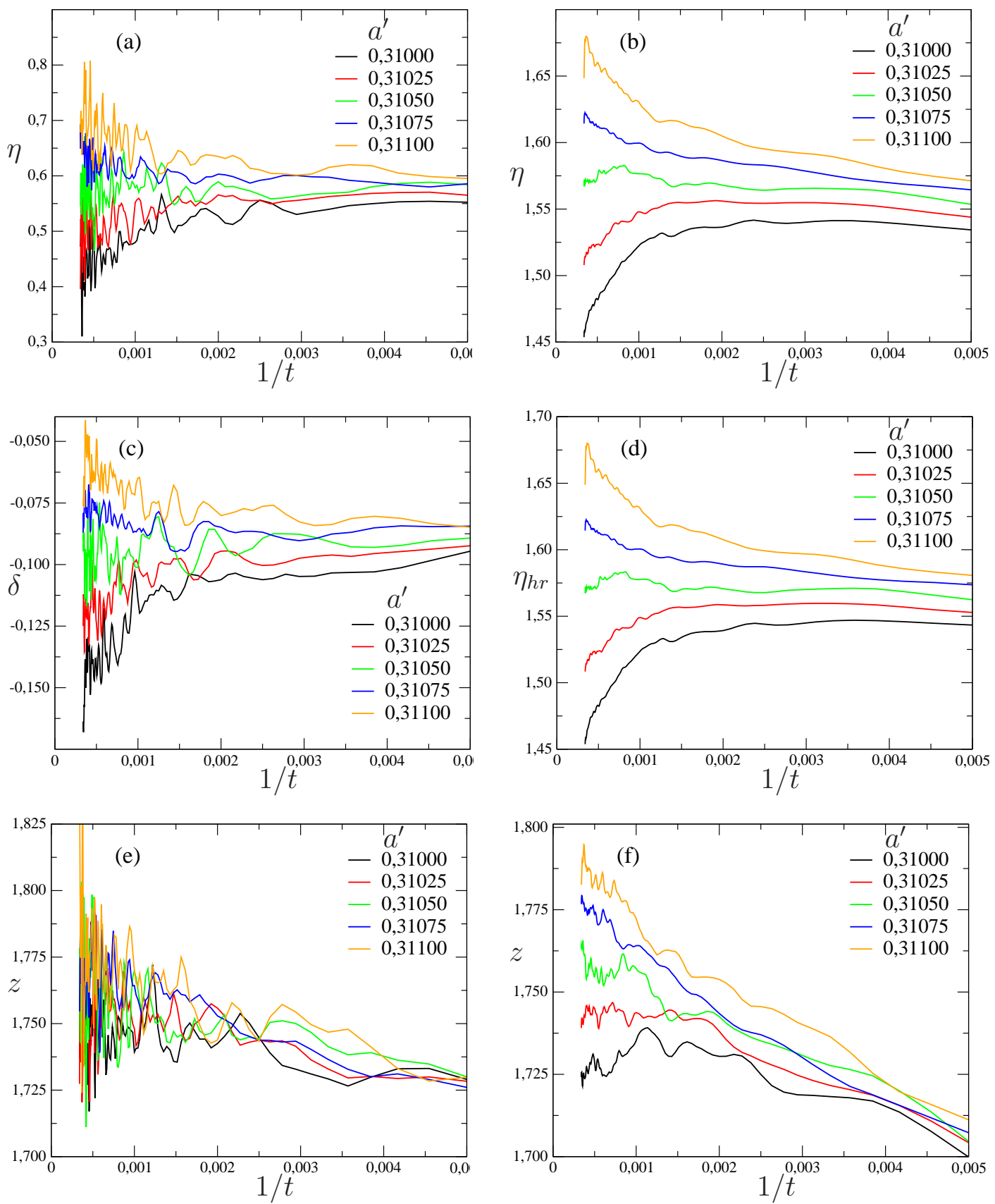

Figura 4.17: Expoentes dinâmicos em função do inverso do tempo para probabilidade de morte dos vetores $e=0$ para diferentes grandezas. Expoente dinâmico $\eta$ associado ao número de hospedeiros (a) e vetores (b) infectados. Expoente dinâmico $\delta$ associado à probabilidade de sobrevivência da epidemia na população de hospedeiros (c) e expoente associado ao número de hospedeiros recuparados (d). Expoente dinâmico z associado ao raio quadrático médio de espalhamento da epidemia na subrede de hospedeiros (e) e vetores (f). 


\subsection{Diagrama de fase do modelo}

A partir dos dados da tabela 4.3, na qual consta a síntese dos resultados obtidos por meio das simulações dependentes do tempo, construiu-se o diagrama de fase em termos das probabilidades de morte do vetor e da probabilidade de infecção. Na figura 4.18, tem-se a linha crítica obtida por meio da aproximação de campo médio simples, por pares e por meio de simulações dependentes do tempo. Nas simulações, o limiar de infecção foi determinado analisando os expoentes dinâmicos em função do tempo, observando qual probabilidade corresponde à curvas com expoentes dinâmicos com inclinação constante no tempo. Acima da linha crítica, há propagação da epidemia e, assim, o estado absorvente contêm hospedeiros suscetíveis e recuperados, bem como vetores suscetíveis. Abaixo da linha crítica, a epidemia não se espalha e, por isso, o estado absorvente contem somente hospedeiros e vetores suscetíveis. No limite que $e=0$, o limiar de infecção é $a_{c}=1 / 4$ na aproximação de campo médio por pares e nas simulações dependentes do tempo $a_{c}=0,31050(3)$. Abaixo da linha crítica, tem-se a fase sem propagação da epidemia, cujo estado estacionário é formado por vetores e hospedeiros suscetíveis. Acima da linha crítica, a epidemia dura um tempo longo e, por isso, a densidade de hospedeiros recuperados é sempre não-nula. A diagonal $c=0$ delimita o espaço de probabilidades.

Neste diagrama, para $e \rightarrow 0$, o aumento da probabilidade de morte dos vetores provoca um aumento no limiar de infecção. Isso parece contraditório, pois exterminar vetores usualmente reduz as chances de haver epidemia. Contudo, não se deve ignorar a condição de normalização que adotada $(2 a+e+c=1)$. Ao variar as probabilidades $a$ e $e$, está-se variando

$c$ também. Próximo de $e=0$, à medida que se aumenta $e$, o limiar de infecção $a_{c}$ atinge um máximo e depois tende a zero, seguindo a assíntota $\mathrm{c}=0$. Para enfatizar que o crescimento da probabilidade de infecção para $e$ pequeno deve-se à probabilidade $c$, construi-se outro diagrama de fase em termos das probabilidades relativas $a / c$ e $e / c$. Na figura 4.19, está inscrita a linha crítica em termos das probabilidades relativas $a / c$ versus $e / c$, que permite observar que o limiar de infecção relativo cresce à medida que se aumenta o controle vetorial $e / c$.

Ademais, em ambos os diagramas de fase, a linha crítica obtida por meio da aproximação de campo médio por pares reproduz qualitativamente os resultados obtidos via simulações computacionais do modelo. Ao longo de toda linha crítica, espera-se que os expoentes críticos dinâmicos correspondam aos expoentes críticos da classe de universalidade da percolação dinâmica. 


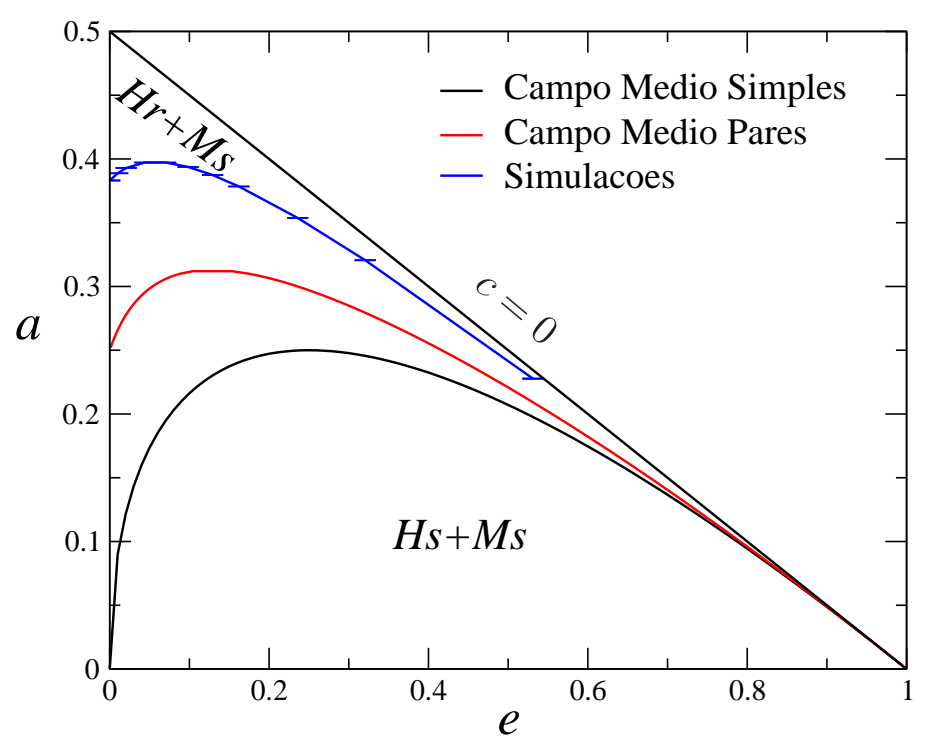

Figura 4.18: Diagrama de fase do modelo de infecção cruzada. A linha de transição de fase foi obtida por meio de aproximação de campo médio simples, por pares e por simulações computacionais dependentes do tempo.

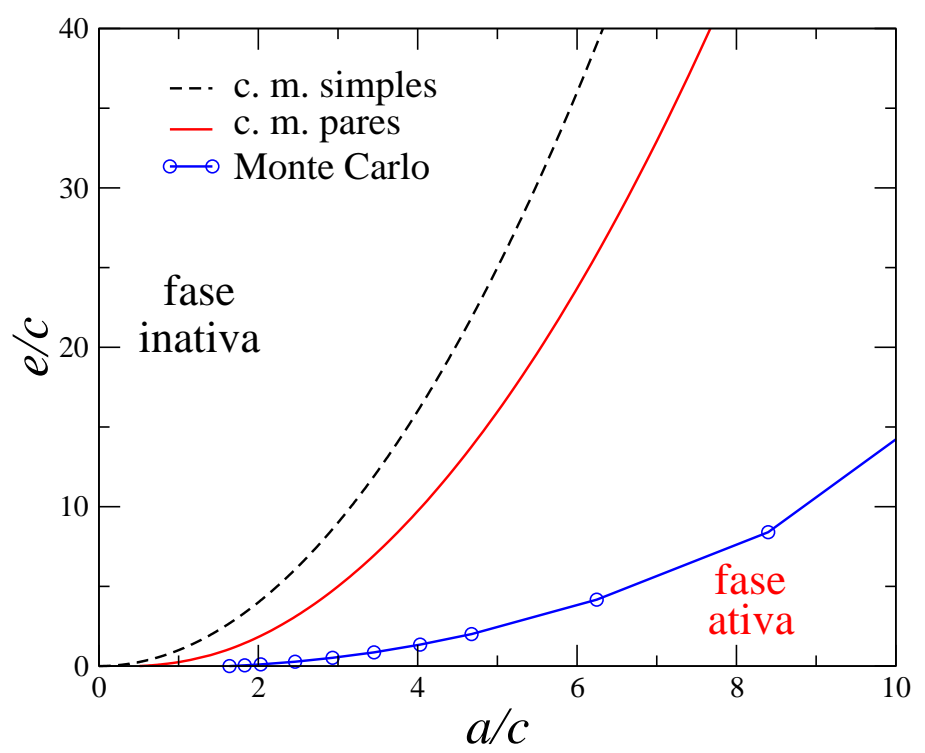

Figura 4.19: Diagrama de fase do modelo de infecção cruzada em termos das probabilidades relativas $a / c$ e $e / c$. A linha crítica foi determinada por meio de simulações dependentes do tempo, aproximações de campo médio simples e por pares. Na fase ativa a epidemia pode se espalhar, enquanto na fase inativa a epidemia cessa em um tempo muito curto, sem haver espalhamento da epidemia. 


\section{Capítulo 5}

\section{Modelo de infecção cruzada II}

\subsection{Descrição do modelo}

O modelo de infecção cruzada no capítulo 4 descreve a propagação de uma doença entre duas espécies. O modelo descreve a existência de uma fase sem propagação de doença e outra fase com ocorrência de epidemia. Contudo, ambas as fases apresentam estados estacionários absorventes a subrede de vetores ocupada por vetores suscetíveis. Isso significa que, neste estado estacionário, a sub-rede é totalmente ocupada por vetores suscetíveis, independentemente do controle de natalidade/mortalidade, e não existe uma densidade limite de vetores necessária para haver dispersão da doença.

Buscando obter resultados que descrevam de forma mais clara a importância do controle vetorial, introduziu-se um novo modelo de infecção cruzada. Este modelo difere do anterior pela existência de um estado vazio com reações locais que representam explicitamente a morte e o nascimento de vetores.

Considerou-se que um vetor pode estar suscetível, infectado ou morto (ausência de vetor). Quando um vetor suscetível ou infectado morre, dá origem a um espaço vazio onde posteriormente pode nascer um novo indivíduo suscetível. Conforme ilustrado na figura 5.1, associou-se a cada sítio uma variável estocástica $\eta_{i}$ que pode assumir um dos seguintes valores: $\eta_{i}=0,1$ ou 2, respresentando a ausência de vetor, a presença de um vetor suscetível, ou a presença de um vetor infectado, respectivamente. Esta variável pode representar também o estado de saúde de um hospedeiro, o qual pode assumir um dos seguintes estados: suscetível $\left(\eta_{i}=3\right)$, infectado $\left(\eta_{i}=4\right)$ e recuperado/imune $\left(\eta_{i}=5\right)$. As regras de transição local entre vetores são similares às do modelo suscetível-infectado-recuperado (SIRS) [34,54,55] com a adição de uma reação reversa para uma das transições.

Conforme ilustrado na figura [5.1, assumiu-se que, tanto os vetores 
suscetíveis quanto os infectados, morrem com probabilidade $e$. Este processo é espontâneo e representa a morte dos vetores devido aos processos naturais e controle de zoonoses. Se o sítio estiver vazio, então pode espontaneamente tornar-se ocupado por um vetor suscetível como probabilidade $r$. O processo de infecção é catalítico e pode provocar a infecção de um vetor/hospedeiro suscetível se houver pelo menos um hospedeiro/vetor infectado na vizinhança do sítio. A probabilidade de infecção é proporcional ao número de primeiros vizinhos infectados. Uma vez infectado, o hospedeiro pode recuperar-se espontaneamente com probabilidade $c$.
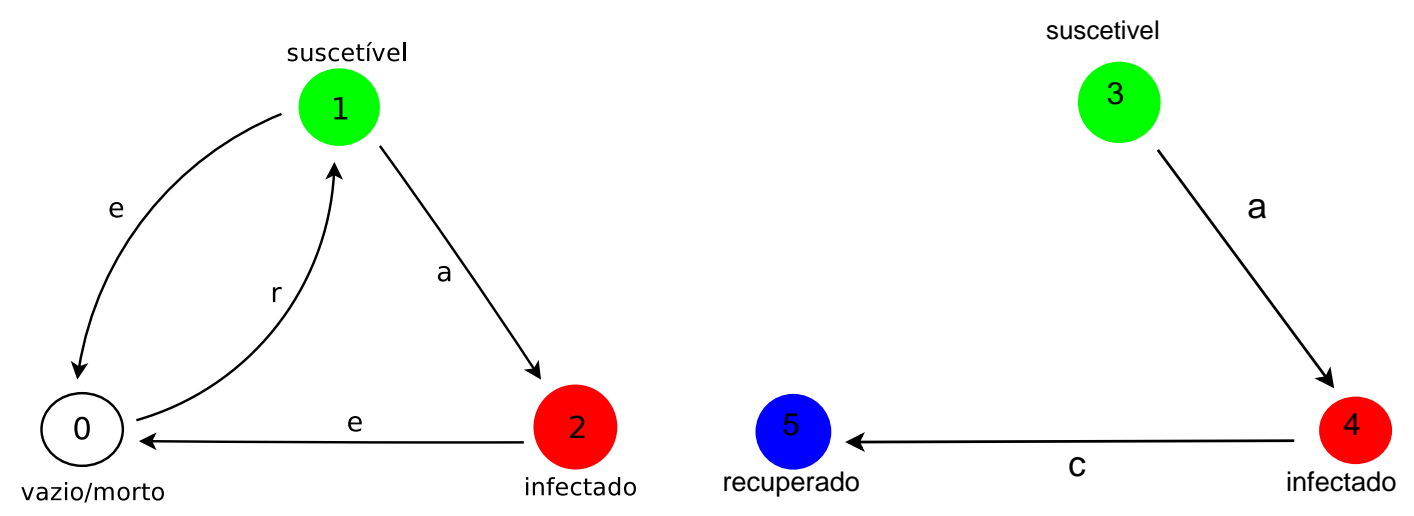

Figura 5.1: Regras de transição para o modelo. Os parâmetros $a, c$, e e $r$ representam as probabilidades de transição e os números são os valores associados à variável estocástica para denotar o estado do sítio. O processo de infecção cruzada é catalítico e as demais transições são espontâneas.

Dependendo dos valores de cada uma das probabilidades, a introdução de único indivíduo infectado (vetor ou hospedeiro) pode produzir uma epidemia. Abaixo de certo limiar de infecção, a epidemia cessa em um intervalo muito curto, de forma que se $\left(a<<a_{c}\right)$ então a densidade de indivíduos afetados pela doença tende a um valor nulo.

Para determinar os limiares de infecção, investigou-se este modelo por meio de aproximação de campo médio e simulações de Monte Carlo. Por meio de simulações, será analisado o comportamento crítico de diferentes grandezas, buscando determinar os expoentes críticos dinâmicos, a fim de determinar a classe de universalidade do modelo. 


\subsection{Equação mestra}

Considerando um reticulado com $N$ sítios, em que cada sítio denota um vetor ou hospedeiro, é possível representar uma configuração do sistema pelo vetor (matriz linha)

$$
\eta=\left(\eta_{1}, \eta_{2}, \ldots, \eta_{i}, \ldots, \eta_{N}\right),
$$

em que $\eta_{i}$ é uma variável estocástica associada ao i-ésimo sítio e que pode assumir um dos seguintes valores: $\eta_{i}=0$ (espaço vazio), $\eta_{i}=1$ (vetor suscetível), $\eta_{i}=2$ (vetor infectado), $\eta_{i}=3$ (hospedeiro suscetível), $\eta_{i}=4$ (hospedeiro infectado) e $\eta_{i}=5$ (hospedeiro recuperado).

As probabilidades de transição esboçadas na figura 5.1 podem ser escritas como segue:

$$
\begin{array}{rll}
w_{i}^{01}=r \quad \text { se } & \eta_{i}=0 \\
w_{i}^{12}(\eta)=\frac{a}{\zeta} \sum_{\delta}\left(\eta_{i+\delta}, 4\right) & \text { se } \quad \eta_{i}=1 \\
w_{i}^{10}(\eta)=e \quad \text { se } & \eta_{i}=1 \\
w_{i}^{20}(\eta)=e \quad \text { se } & \eta_{i}=2 \\
w_{i}^{34}(\eta)=\frac{a}{\zeta} \sum_{\delta}\left(\eta_{i+\delta}, 2\right) & \text { se } \quad \eta_{i}=3 \\
w_{i}^{45}(\eta)=c \quad \text { se } & \eta_{i}=4
\end{array}
$$

em que $w_{i}^{\alpha \beta}(\eta)$ denota a probabilidade de transição de $\alpha \rightarrow \beta$. As outras probabilidades de transição são nulas.

Na equação mestra, considerando que um único sítio é atualizado por vez, a evolução temporal de uma função de estado pode ser calculada usando a expressão:

$$
\left.\frac{d}{d t}\langle f(\eta)\rangle=\sum_{i=1}^{N} \sum_{\alpha \beta}\left\langle\left[f\left(A_{i}^{\alpha \beta} \eta\right)-f(\eta)\right] w_{i}^{\alpha \beta} \eta\right)\right\rangle,
$$

onde $A_{i}^{\alpha \beta} \eta$ denota o estado $\eta^{\prime}$ que se obtem de $\eta$ da seguinte forma. Se $\eta_{i}=\alpha$ entao $\eta_{i}^{\prime}=\beta$. Se $\eta_{i} \neq \alpha$ o estado não é modificado, isto é, $\eta_{i}^{\prime}=\eta_{i}$.

Para se obterem as equações de evolução das densidades, deve-se substituir $f(\eta)$ pela probabilidade do i-ésimo sítio estar em determinado estado. Por exemplo, com $f(\eta)=\delta\left(\eta_{i}, 1\right)$, pode-se obter a equação de evolução da densidade de vetores suscetíveis. Como procedimentos similares foram feitos para outros modelos (capítulo II e III), serão apresentados somente as equações, e não o desenvolvimento delas.

As equações a seguir representam a evolução da densidade de sítios vazios $P(0)$, vetores suscetíveis $P(1)$, vetores infectados $P(2)$, hospedeiros suscetíveis 
$P(3)$, infectados $P(4)$ e recuperados $P(5)$, derivadas a partir da equação mestra usando atualização assíncrona.

$$
\begin{aligned}
\frac{d P(0)}{d t} & =e[P(1)+P(2)]-r P(0), \\
\frac{d P(1)}{d t} & =r P(0)-e P(1)-a P(14) \\
\frac{d P(2)}{d t} & =a P(14)-e P(2), \\
\frac{d P(3)}{d t} & =-a P(32), \\
\frac{d P(4)}{d t} & =a P(32)-c P(4), \\
\frac{d P(5)}{d t} & =c P(4) .
\end{aligned}
$$

O sistema de equações (5.1) é um conjunto de equações diferenciais acopladas que aparentemente não apresenta uma solução exata. Pode-se fazer aproximações que possibilitem descobrir o comportamento das densidades em função das probabilidades de transição local. Na seção 5.3, será empregada a aproximação de campo médio simples e, na seção [5.4, a aproximação de campo médio por pares para se obter a linha de transição de fase.

\subsection{Aproximação de campo médio simples}

No sistema de equações (5.1), será aplicada a aproximação de campo médio simples para descorrelacionar as probabilidades que envolvem dois sítios. Para facilitar a notação, utilizaram-se as seguintes definições: $w=$ $P(0), u=P(1), v=P(2), x=P(3), y=P(4)$ e $z=P(5)$, com as condições de normalização:

$$
x+y+z=1,
$$

e

$$
w+u+v=1
$$

Devido a essas condições de normalização, pode-se escolher quatro densidades independentes. Foram escolhidas $x, y, v$ e $w$ como densidades independentes. Com essa escolha e aplicando-se a aproximação de campo médio simples, 
encontrou-se que o sistema de equações (5.1) torna-se

$$
\begin{aligned}
\frac{d w}{d t} & =e(1-w)-r w=e-w(e+r), \\
\frac{d v}{d t} & =a y(1-v-w)-e v, \\
\frac{d x}{d t} & =-a v x, \\
\mathrm{e} \quad \frac{d y}{d t} & =a v x-c y .
\end{aligned}
$$

As probabilidades de infecção, morte, recuperação e nascimento podem ser normalizadas pela condição:

$$
2 a+2 e+r+c=1 .
$$

Na figura 5.2, é demonstrada a evolução da densidade de infectados em função do tempo para as probabilidades $(a, e, r)=(0,25 ; 0,10 ; 0,10)$ obtidas pela integração numérica do sistema de equações (5.4), usando $v(t=0)=$ $10^{-4}$

No estado estacionário, o sistema de equações (5.4) apresenta o ponto fixo:

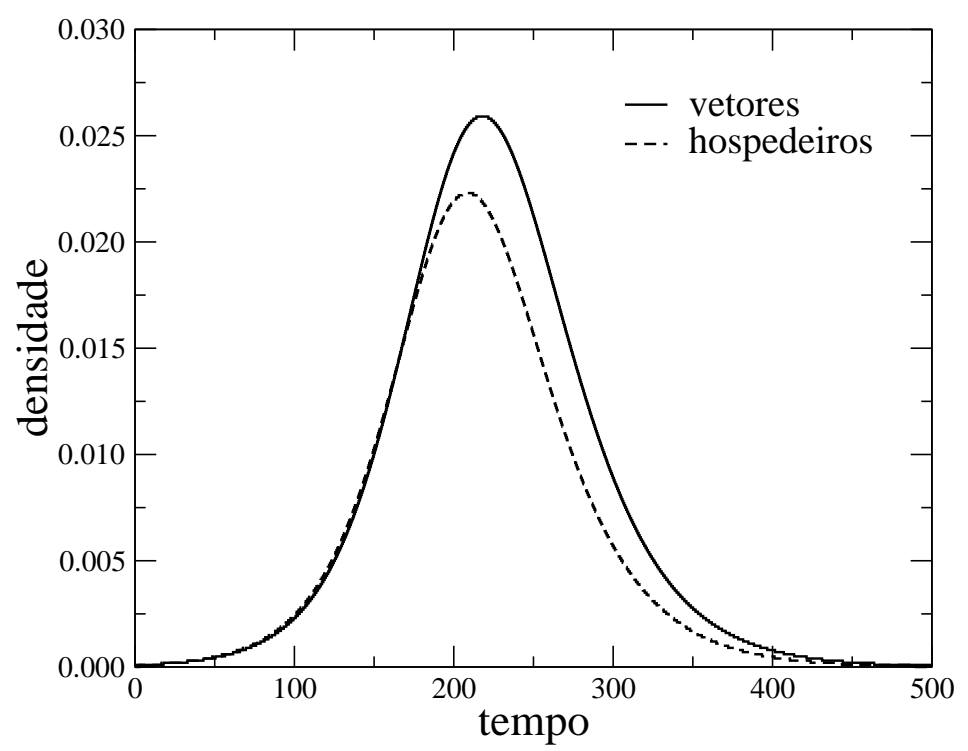

Figura 5.2: Densidade de vetores e hospedeiros infectados em função do tempo para $(a, e, r)=(0,25 ; 0,10 ; 0,10)$.

$(x, y, v, w)=(1,0,0, e /(e+r))$, que representa um estado absorvente de hospedeiros suscetíveis e vetores suscetíveis e vazios (ausencia de vetores). A 
densidade estacionária de vetores suscetíveis é $u^{*}=r /(e+r)$ e a densidade de sítios vazios é $w^{*}=e /(e+r)$, que dependem somente da natalidade e mortalidade de vetores.

No ponto fixo, o jacobiano do sistema de equações (5.4) torna-se:

$$
\mathbf{J}=\left(\begin{array}{cccc}
0 & 0 & -a & 0 \\
0 & -c & a & 0 \\
0 & a\left(1-\frac{e}{e+r}\right) & -e & 0 \\
0 & 0 & 0 & -(e+r)
\end{array}\right)
$$

Pela equação de autovalores $(\operatorname{det}(J-\lambda I)=0)$, pode-se calcular os autovalores e, com eles, determinar os autovetores. Entretanto, o interesse é caracterizar a estabilidade do ponto fixo. Para isso, impõe-se $\lambda=0$ na equação de autovalores:

$$
-e c(e+r)+a^{2}(e+r)\left(1-\frac{e}{e+r}\right)=(e+r)\left[-e c+a^{2} \frac{e}{e+r}\right]=0 .
$$

Como $e+r$ deve ser diferente de zero, é necessário que

$$
-e c+a^{2} \frac{e}{e+r}=0 .
$$

A equação (5.7) é a superfície crítica que separa a fase sem espalhamento da doença (densidade de hospedeiros suscetíveis $x=1$ ) da fase com espalhamento que apresenta densidade não-nula de recuperados $(x<1 \mathrm{e}$ $z>0)$. 


\subsection{Aproximação de campo médio por pares}

É possível melhorar a aproximação de campo médio feita para o sistema de equações (5.1) calculando as densidades envolvendo correlação entre dois sítios. As densidades de pares seguem as seguintes condições de normalização:

$$
\begin{aligned}
& P(03)+P(04)+P(05)=P(0), \\
& P(13)+P(14)+P(15)=P(1), \\
& P(23)+P(24)+P(25)=P(2),
\end{aligned}
$$

com $P(0)+P(1)+P(2)=1$ e $P(3)+P(4)+P(5)=1$.

Entre essas probabilidades, pode-se escolher $P(13), P(23), P(14), P(24)$, $P(1), P(2), P(3)$ e $P(4)$ como probabilidades independentes. Assim, as equações de evolução para esses momentos da distribuição são:

$$
\begin{aligned}
\frac{d P(1)}{d t} & =r P(0)-e P(1)-a P(14) \\
\frac{d P(2)}{d t} & =a P(14)-e P(2) \\
\frac{d P(3)}{d t} & =-a P(32) \\
\frac{d P(4)}{d t} & =a P(32)-c P(4) \\
\frac{d P(13)}{d t} & =r P(03)-e P(13)-a \frac{\zeta-1}{\zeta}[P(413)+P(132)] \\
\frac{d P(23)}{d t} & =-\left(e+\frac{a}{\zeta}\right) P(23)+a \frac{\zeta-1}{\zeta}[P(413)-P(232)] \\
\frac{d P(14)}{d t} & =-\left(e+\frac{a}{\zeta}+c\right) P(14)+r P(04)-a \frac{\zeta-1}{\zeta}[P(414)-P(132)]
\end{aligned}
$$

$\mathrm{e}$

$\frac{d P(24)}{d t}=\frac{a}{\zeta}[P(14)+P(23)]-(e+c) P(24)+a \frac{\zeta-1}{\zeta}[P(414)+P(232)]$,

em que $\zeta$ é a coordenação da rede.

Aplicando-se a aproximação de campo médio por pares, os termos que dependem de trios como $P(132)$ são aproximados usando a relação $P(\alpha \beta \gamma)=P(\alpha \beta) P(\beta \gamma) / P(\beta)$. Com essa aproximação, é possível simplificar a notação definindo as seguintes densidades: $x=P(3), y=P(4), u=P(1)$, $v=P(2), m=P(13), n=P(23), s=P(14)$ e $p=P(24)$, com as quais o 
sistema de equações (5.11) pode ser reescrito, tornando-se:

$$
\begin{aligned}
\frac{d u}{d t} & =r-(r+e) u-r v-a s, \\
\frac{d v}{d t} & =a s-e v, \\
\frac{d x}{d t} & =-a n, \\
\frac{d y}{d t} & =a n-c y, \\
\frac{d m}{d t} & =r x-(r+e) m-r n-a \frac{\zeta-1}{\zeta} m\left(\frac{s}{u}+\frac{n}{x}\right), \\
\frac{d n}{d t} & =-\left(e+\frac{a}{\zeta}\right) n+a \frac{\zeta-1}{\zeta}\left(\frac{s m}{u}-\frac{n^{2}}{x}\right), \\
\frac{d s}{d t} & =-\left(e+c+r+\frac{a}{\zeta}\right) s+r(y-p)-a \frac{\zeta-1}{\zeta}\left(\frac{s^{2}}{u}-\frac{m n}{x}\right), \\
\frac{d p}{d t} & =\frac{a}{\zeta}(s+n)-(c+e) p+a \frac{\zeta-1}{\zeta}\left(\frac{s^{2}}{u}+\frac{n^{2}}{x}\right) .
\end{aligned}
$$

No estado estacionário, este sistema apresenta os seguintes pontos fixos $P(1)=\frac{r}{r+e}, P(0)=1-P(1), P(2)=0, P(3)=1, P(4)=P(5)=P(23)=$ $P(14)=P(24)=0$ e $P(13)=\frac{r}{r+e}$, que para as variáveis adotadas, escrevese como $\left(x^{*}, y^{*}, u^{*}, v^{*}, m^{*}, n^{*}, s^{*}, p^{*}\right)=\left(1,0, \frac{r}{r+e}, 0, \frac{r}{r+e}, 0,0,0\right)$, e representa um estado absorvente de indivíduos suscetíveis. Além deste ponto, tem-se outro estado possível, com densidade não-nula de hospedeiros recuperados mas diferente da unidade, que pode ser representado como $P(3)=\beta$, sendo $P(4)=0$ e $P(5)=1-\beta$, em que $\beta$ denota a densidade de hospedeiros suscetíveis e $1-\beta$, a densidade de hospedeiros recuperados. Este ponto fixo corresponde ao estado com espalhamento da epidemia. No ponto fixo, a matriz jacobiana do sistema de equações (5.12) é:

$$
\left(\begin{array}{cccccccc}
0 & 0 & 0 & 0 & 0 & -a & 0 & 0 \\
0 & -c & 0 & 0 & 0 & a & 0 & 0 \\
0 & 0 & -(r+e) & -r & 0 & 0 & -a & 0 \\
0 & 0 & 0 & -e & 0 & 0 & a & 0 \\
r & 0 & 0 & 0 & -(r+e) & -r-a \frac{\zeta-1}{\zeta} \frac{r}{r+e} & -a \frac{\zeta-1}{\zeta} & 0 \\
0 & 0 & 0 & 0 & 0 & -(e+a / \zeta) & a \frac{\zeta-1}{\zeta} & 0 \\
0 & r & 0 & 0 & 0 & a \frac{\zeta-1}{\zeta} \frac{r}{r+e} & -\left(e+c+r+\frac{a}{\zeta}\right) & -r \\
0 & 0 & 0 & 0 & 0 & \frac{a}{\zeta} & \frac{a}{\zeta} & -(c+e)
\end{array}\right)
$$

Escrevendo a equação de autovalores $(\operatorname{det}[J-\lambda I]=0)$, em que $J$ é a matriz 
jacobiana e $\lambda$ são os autovalores, tem-se a equação a seguir:

$$
\begin{aligned}
& -(\lambda+c)(-1)^{1+1}\left\{-\left(\lambda+\frac{a}{\zeta}+e\right)\left(\lambda+\frac{a}{\zeta}+c+e+r\right)(\lambda+c+e)-\right. \\
& \left.-r a^{2} \frac{\zeta-1}{\zeta^{2}}-r \frac{a}{\zeta}\left(\lambda+\frac{a}{\zeta}+e\right)+\frac{r}{r+e}\left(a \frac{\zeta-1}{\zeta}\right)^{2}(\lambda+c+e)\right\}+ \\
& +r(-1)^{3+1}\left\{-a^{2} \frac{\zeta-1}{\zeta}(\lambda+c+e)\right\}=0
\end{aligned}
$$

Como o interesse é descobrir onde há perda de estabilidade, pode-se impor $\lambda=0$. Com essa condição, a equação (5.13) torna-se:

$$
\begin{aligned}
& c\left\{-\left(\frac{a}{\zeta}+e\right)\left(\frac{a}{\zeta}+c+e+r\right)(c+e)-r a^{2} \frac{\zeta-1}{\zeta^{2}}-r \frac{a}{\zeta}\left(\frac{a}{\zeta}+e\right)\right. \\
& \left.+\frac{r}{r+e}\left(a \frac{\zeta-1}{\zeta}\right)^{2}(c+e)\right\}-r\left\{-a^{2} \frac{\zeta-1}{\zeta}(c+e)\right\}=0 .
\end{aligned}
$$

Na figura 5.3, observa-se o diagrama de fase obtido por meio de aproximação de campo médio simples, cuja linha ou superfície crítica é dada pela equação (5.7) e, por meio da aproximação de pares, que tem como resultado a linha ou superfície crítica dada pela equação (5.14) com a condição de normalização $2 a+2 e+c+r=1$, fixando $r=0,100$.
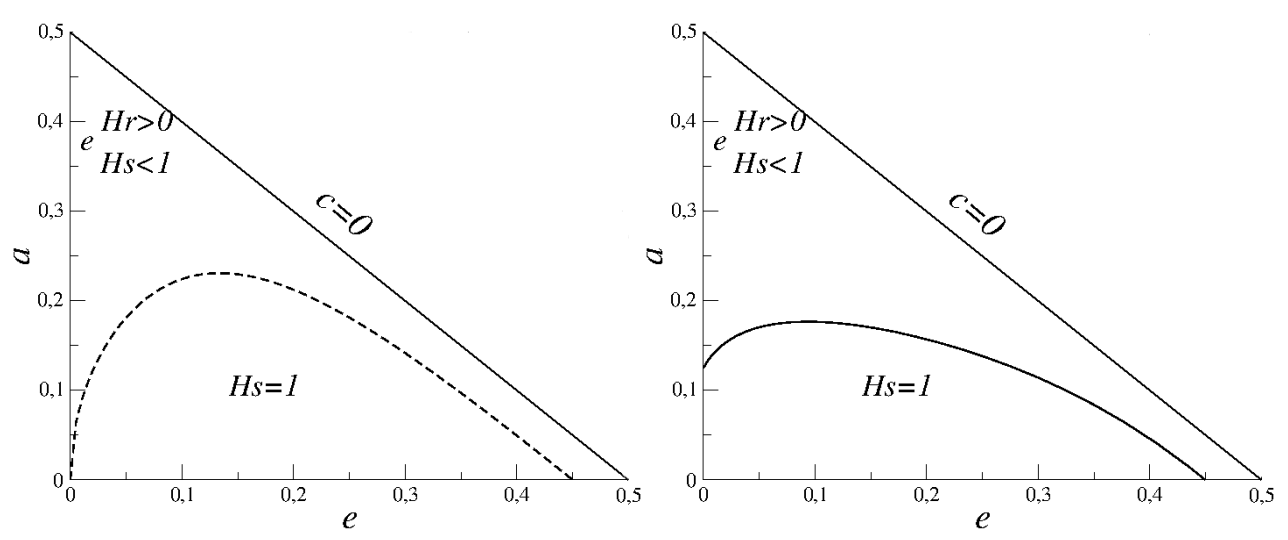

Figura 5.3: Diagrama de fase do modelo obtido via aproximações de campo médio simples (à esquerda) e por pares (à direita) no plano a versus e.

Posteriormente, será feita uma comparação destes resultados aos resultados de simulações de Monte Carlo. 


\subsection{Simulações estacionárias}

A partir de simulações numéricas do modelo, obtem-se o comportamento estacionário das quantidades médias como a densidade de hospedeiros recuperados e a probabilidade de a epidemia atingir a fronteira da rede. Será investigado o comportamento dessas grandezas como função das probabilidades de transição local e determinados os limiares de infecção em função da probabilidade de morte dos vetores $e$, a qual pode estar associada a um controle vetorial.

Usando as regras de interação local entre os indivíduos e aplicando-se o método de Monte Carlo, construiu-se um algoritmo que permite simular a evolução temporal assíncrona do processo de infecção cruzada com nascimento e morte de vetores.

Sabe-se que a condição inicial e as condições de contorno são fatores relevantes quando analisa-se sistemas de tamanho finito. E, uma vez que este modelo possui vários estados absorventes, deve-se usar sempre a mesma condição inicial para todas as realizações, cada uma delas evoluindo a partir de uma série pseudoaleatória distinta. Serão utilizadas condições periódicas de contorno e cada realização (amostra) evoluirá a partir de um estado com todos os hospedeiros suscetíveis e a sub-rede de vetores totalmente ocupada por vetores suscetíveis, com exceção do sítio central ocupado por um vetor infectado. Este estado inicial representa uma pequena perturbação do estado absorvente de vetores e hospedeiros suscetíveis. A sub-rede de vetores apresenta uma densidade inicial de suscetíveis maior que o valor estacionário $(e /(e+r))$.

Seguindo as regras do modelo, elaborou-se um algoritmo de evolução temporal assíncrona, cujas condições a ser analisadas a cada instante seguem as seguintes etapas:

- 0) Definir e inicializar uma rede regular quadrada de lado $L$, formada por duas subredes $A$ e $B$, com condições periódicas de contorno. Colocar todos os sítios da sub-rede $A$ no estado $\eta_{i}=1$ (vetores suscetíveis), exceto o sítio central, com $\eta_{i}=2$ (vetor infectado). Todos os indivíduos da sub-rede $B$ são inicializados com estado $\eta_{i}=3$ (hospedeiros suscetíveis).

- 1) Sortear um sítio da rede e um número pseudoaleatório $\alpha$ que está contido no intervalo $(0 \leq \alpha<1)$ e atualizar o estado do sítio usando a probabilidade de transição por sítio. Se $\alpha<\omega_{i}\left(\eta_{i}\right)$, a variável estocástica $\eta_{i}$ assume seu próximo estado cíclico.

- 2) Repete-se o processo 1 até não haver mais infectado (vetor ou hos- 
pedeiro) na rede. Se não há mais sítios ativos (infectados), significa que o sistema está preso em um estado absorvente e, portanto, a realização deve ser interrompida.

- 3) Calculam-se as quantidades médias de interesse.

- 4) Sorteia-se uma nova semente para o gerador de números pseudoaleatório e repete-se os passos 1 a 3 tantas vezes necessário para se obterem médias com flutuações com a tolerância desejada.

Na figura 5.4, está descrita uma sequencia de retratos para uma série pseudoaleatória. Em $t=1$, parte dos vetores já morreu e o foco epidêmico ainda está concentrado. À medida que o tempo cresce, a epidemia vai se espalhando, formando um aglomerado de indivíduos recuperados. Após um tempo longo, a epidemia acaba e o sistema fica preso em estado absorvente. O espalhamento da epidemia só ocorre para um valor suficientemente grande da probabilidade de infeç̧ão $a$.

Como serão calculadas as quantidades somente quando a realização atinge um estado absorvente, não é preciso inserir um contador para o tempo. Neste modelo, os estados absorventes são formados por vetores suscetíveis, espaços vazios na sub-rede de vetores e hospedeiros suscetíveis e recuperados. As densidades estacionárias de vetores e sítios vazios estão relacionadas à probabilidade de nascimento e morte dos vetores. Por outro lado, a densidade de hospedeiros recuperados depende da probabilidade de infecção, pois, quanto maior essa probabilidade, maior o número de indivíduos contaminados. Desse modo, nas simulações estacionárias, é suficiente analisar as quantidades associadas à população de hospedeiros. As quantidades calculadas foram as mesmas analisadas no modelo SIR, isto é: o número médio de hospedeiros recuperados

$$
S=<N_{h r}>\text {; }
$$

a densidade de hospedeiros recuperados

$$
\rho=\frac{<N_{h r}>}{N},
$$

em que $N$ é o total de sítios da subrede;

o número quadrático médio de hospedeiros recuperados

$$
M=<N_{r}^{2}>;
$$

a razão entre o número quadrático médio de hospedeiros recuperados e o quadrado do número médio de hospedeiros recuperados

$$
U=\frac{<N_{h r}^{2}>}{\left.<N_{h r}\right\rangle^{2}}=\frac{M}{S^{2}} ;
$$



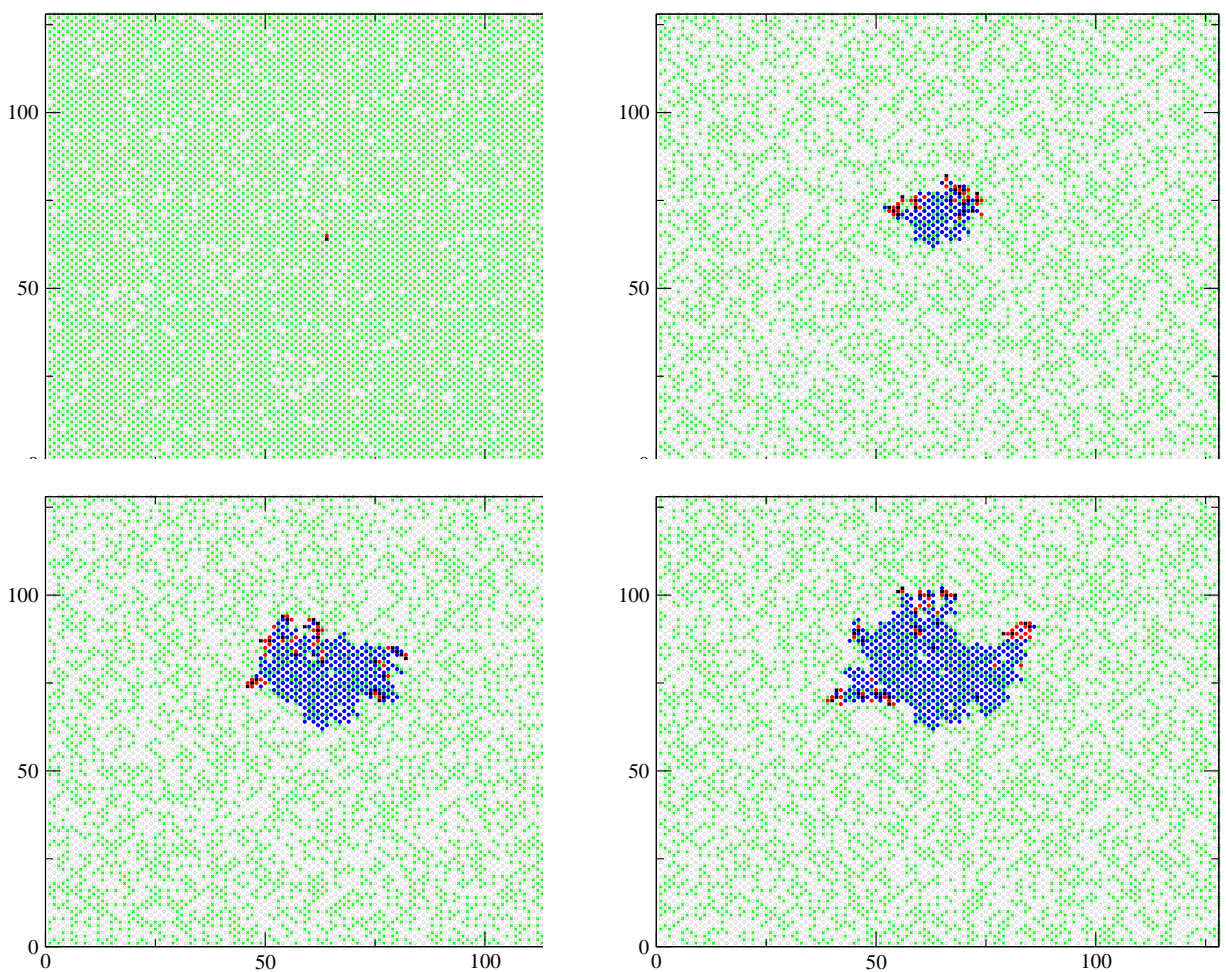

Figura 5.4: Retratos da evolução temporal de uma realização nos instantes $t=1,200,400$ e 500 passos de Monte Carlo. Os parâmetros são $L=64, a=0,3320$ e $e=r=0,1$. Em cada retrato, os pontos branco denotam ausência de vetor, os pontos verde/preto representam vetores suscetíveis/infectados e os pontos marrom/vermelho/azul representam hospedeiros suscetíveis/infectados/recuperados, respectivamente.

e a probabilidade $P$ do aglomerado de hospedeiros recuperados atingir a borda da rede.

Essas grandezas médias foram calculadas para diferentes valores da probabilidade de morte dos vetores e e da probabilidade de infecção $a$. Visando determinar os limiares de infecção, fixou-se a probabilidade de nascimento dos vetores em $r=0,1$ para todas as simulações. Com esta imposição, a equação $2 a+2 e+c+r=1$ fica com duas variáveis independentes. Deixando as probabilidades $a$ e $e$ como variáveis independentes, a probabilidade $c$ fica escrita em função das demais probabilidades. Então, é possível variar a probabilidade $e$ e determinar a probabilidade crítica de infecção $a_{c}$.

Na figura 5.5, constam a densidade $\rho$ de hospedeiros recuperados, a probabilidade $P$ dos aglomerados de recuperados atingir a borda da rede, 
o cumulante $U$ e o produto $U P$ para diferentes tamanhos de rede em função da probabilidade de infecção $a$, mantendo a probabilidade de morte dos vetores $e=0,100$. A densidade de recuperados e a probabilidade $P$ crescem em função do aumento da probabilidade de infecção. Para tamanhos de redes grandes o comportamento dessas grandezas sugere a existência de uma transição de fase contínua, entre uma fase com densidade nula de recuperados e outra fase com densidade não nula de recuperados. A razão entre o número quadrado médio e o número médio ao quadrado de recuperados decai abruptamente em torno do ponto crítico. Na fase supercrítica $a>a_{c}$, à medida que o tamanho da rede aumenta, surgem flutuações relacionadas ao comprimento de correlação espacial.

Conforme discutido para o modelo SIR, o produto $U P$ é uma grandeza universal no ponto crítico, o que significa que, no limiar de infecção, $U P$ independe do tamanho da rede. Portanto, o cruzamento entre as curvas de $U P$ para diferentes tamanhos de rede (visto na figura 5.6) permite estimar o limiar de infecção. Analisando os dados mostrados na figura 5.6, observa-se que para $e=0,100$, o cruzamento das curvas ocorre para $a_{c}=0,3319(1)$.

Neste modelo, devido ao processo de nascimento de vetor, não foi possível fazer otimizações similares ao algoritmo usado no modelo I e, por isso, as simulações são lentas, exigindo grande tempo para realizar cada simulação. Em razão deste fato, o uso da universalidade de $U P$ como critério para determinar o ponto crítico com precisão fica comprometido pela ausência de dados para tamanhos de rede maiores.

Nas figuras 5.7 e 5.8, constam a densidade de recuperados, a probabilidade da epidemia atingir a borda da rede, a razão $U=\frac{M}{S^{2}}$ e o produto $U P$ para as probabilidades de morte dos vetores $e=0,025$ e $e=0,200$, respectivamente. Essas grandezas médias foram calculadas usando $10^{4}$ realizações independentes para todos o tamanhos de rede. Nenhuma das figuras permite determinar o ponto crítico com boa precisão. Para se obter os pontos críticos com melhor precisão, foram realizadas simulações dependentes do tempo, as quais são mais rápidas em comparação às simulações estacionárias e que, adicionalmente, permitem calcular os expoentes críticos dinâmicos e determinar a classe de universalidade do modelo. 

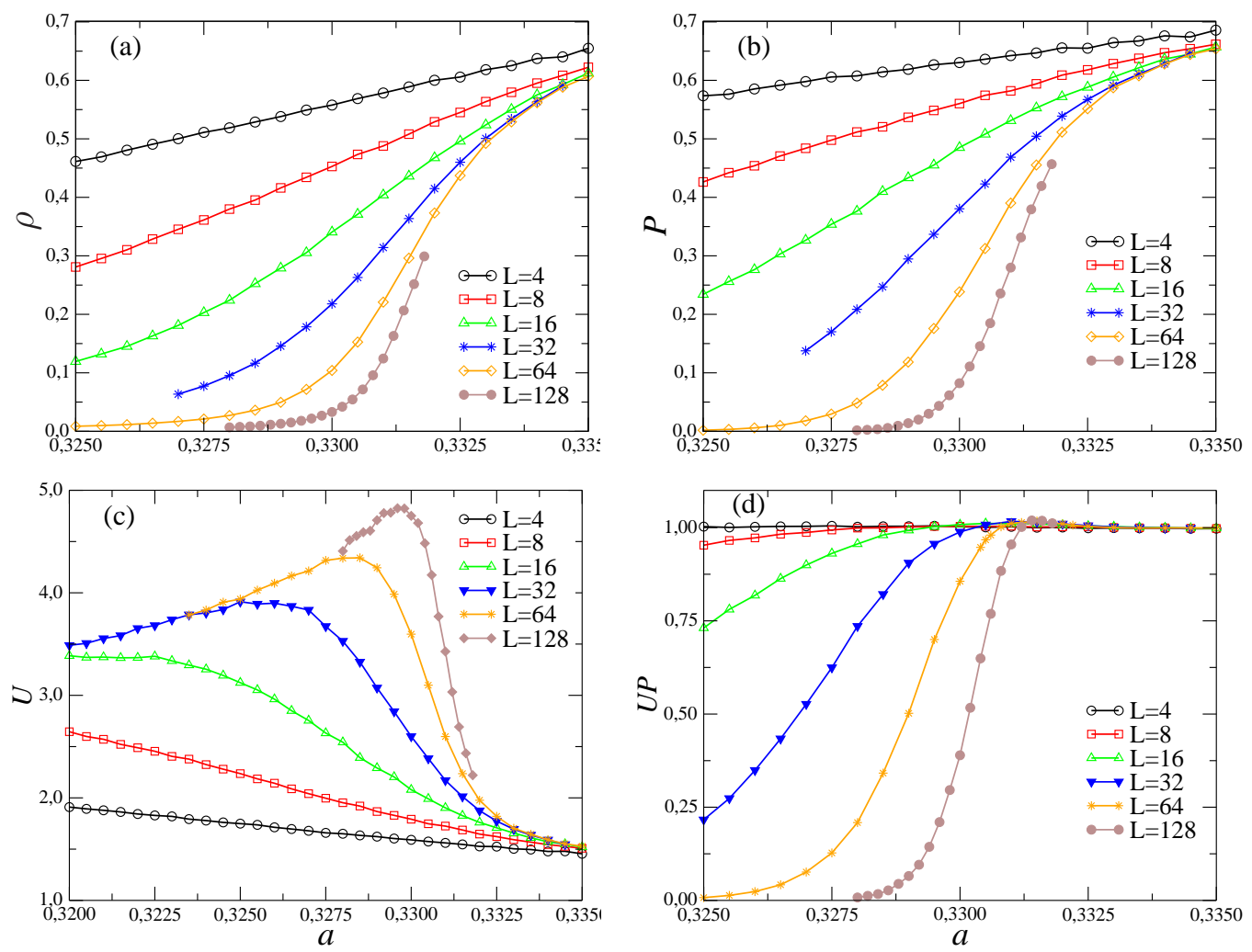

Figura 5.5: Densidade de hospedeiros recuperados $(\rho)$ (a), probabilidade da epidemia atingir a borda da rede $(P)(\mathrm{b})$, razão $U=M / S^{2}(\mathrm{c})$, em que $M$ é o número quadrático médio de recuperados e $S$, o número médio de recuperados, e o produto entre as quantidades $U$ e $P(\mathrm{~d})$ versus a probabilidade de infecção $a$ para diferentes tamanhos de rede quadrada de lado $L$. A probabilidade de morte dos vetores foi fixada em $e=0,1000$, bem como a probabilidade de nascimento $r=0,100$. 


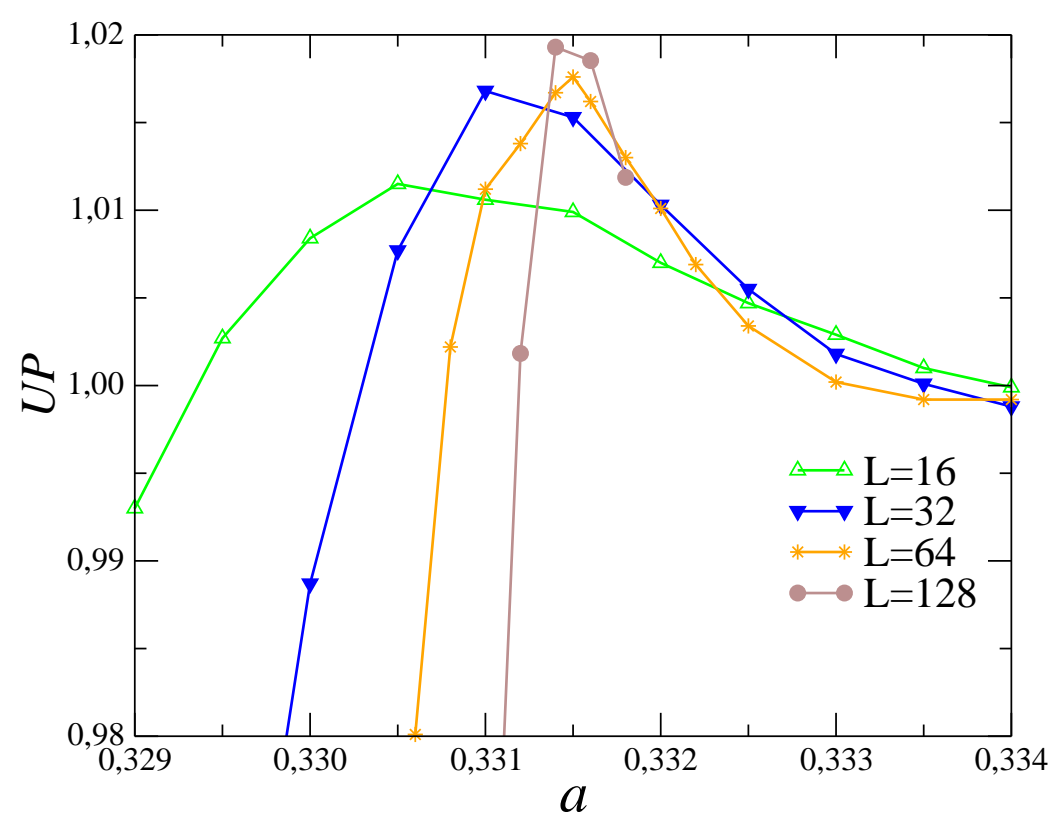

Figura 5.6: Comportamento de $U P$ em função da probabilidade de infecção $a$ para diferentes tamanhos de rede, com probabilidade de morte dos vetores fixa $e=0,100$. No ponto crítico, $U P$ é independente do tamanho da rede e, portanto, as diferentes curvas se cruzam (desde que o tamanho da rede $L$ seja relativamente grande). 

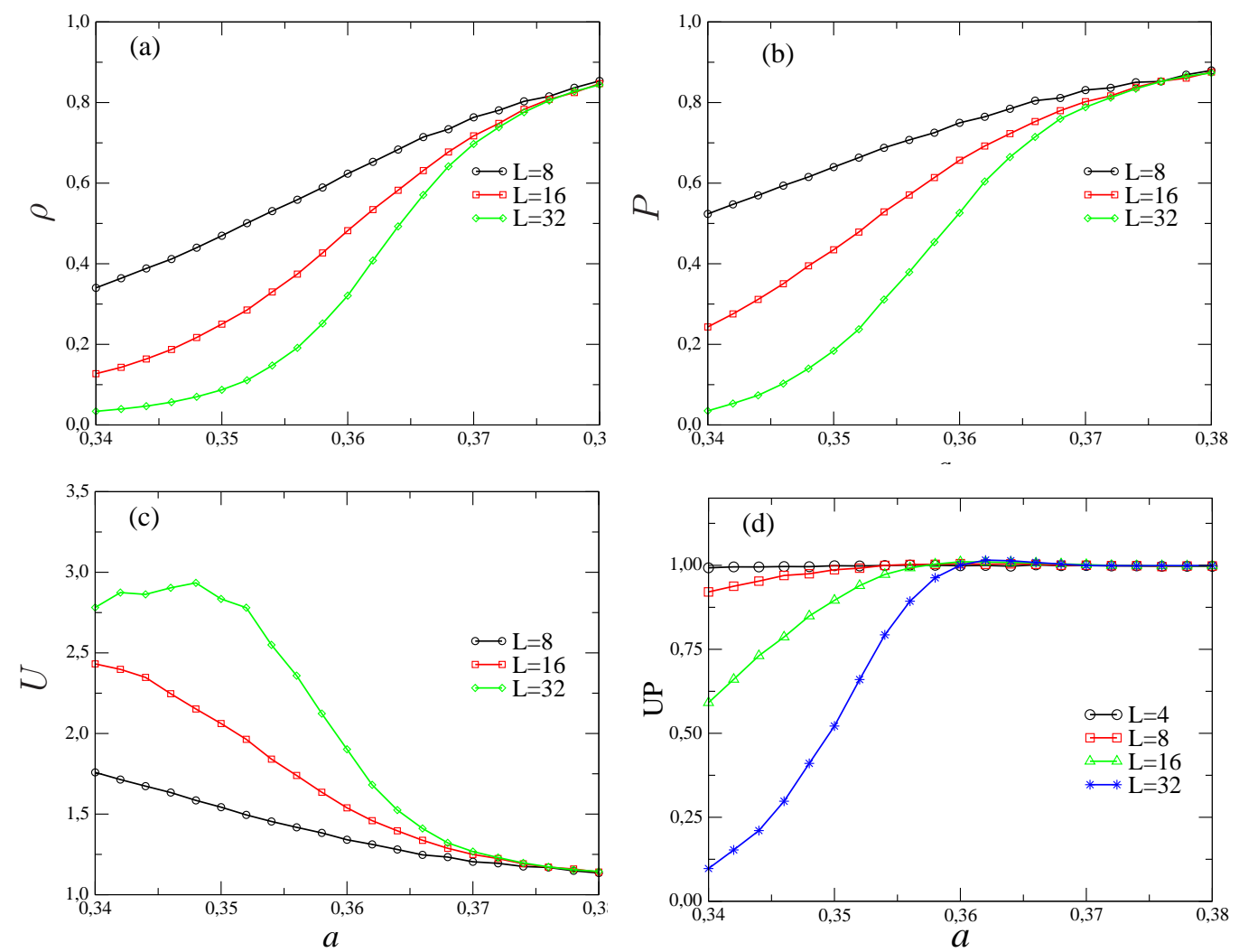

Figura 5.7: Densidade de hospedeiros recuperados $(\rho)$ (a), probabilidade da epidemia atingir a borda da rede $(P)(\mathrm{b})$, razão $U=M / S^{2}(\mathrm{c})$, em que $M$ é o número quadrático médio de recuperados e $S$, o número médio de recuperados, e o produto entre as quantidades $U$ e $P(\mathrm{~d})$ versus a probabilidade de infecção a para diferentes tamanhos de rede quadrada de lado $L$. As probabilidades de morte e nascimento de vetores são $e=0,025$ e $r=0,1000$, respectivamente. 

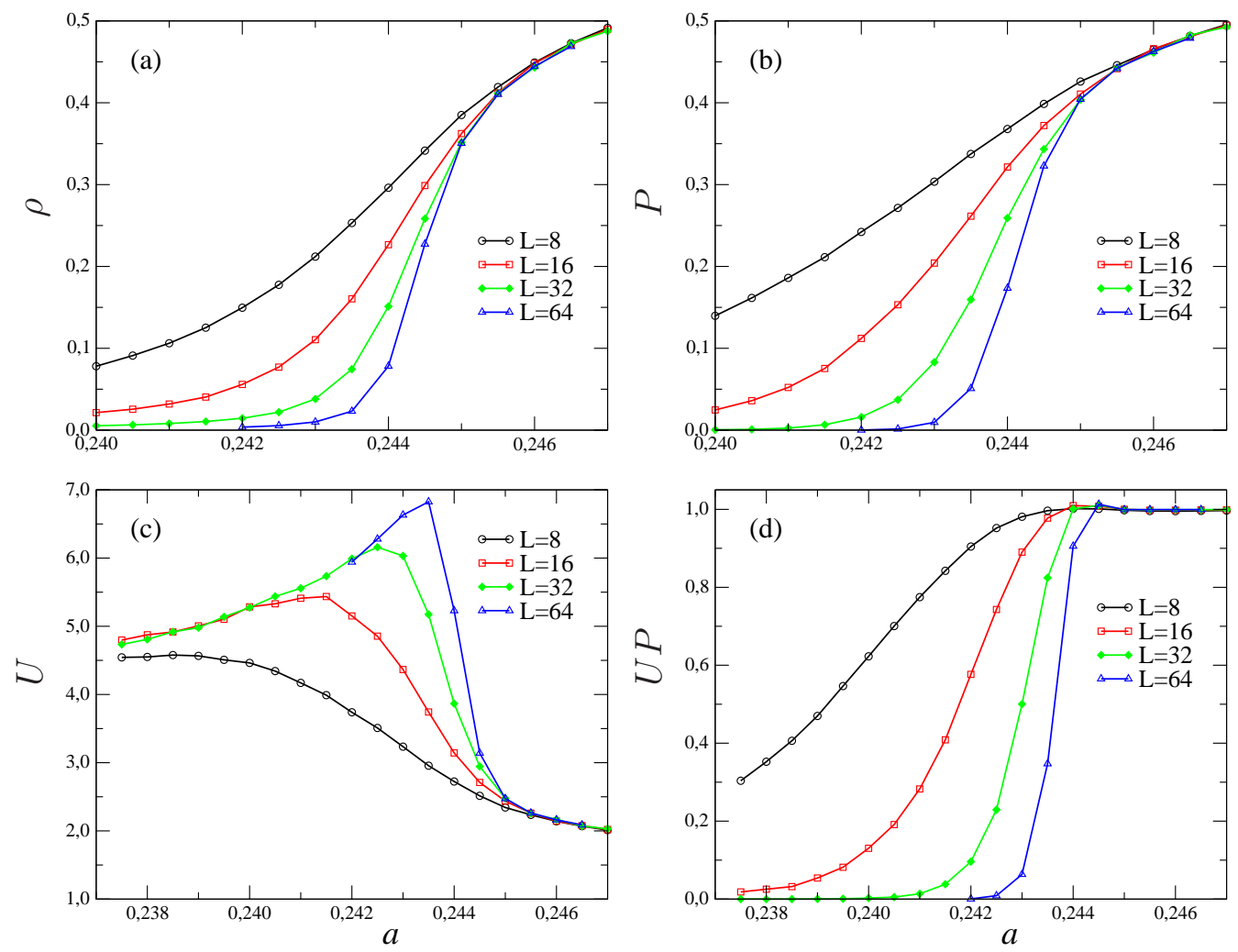

Figura 5.8: Densidade de hospedeiros recuperados $(\rho)$ (a), probabilidade da epidemia atingir a borda da rede $(P)(\mathrm{b})$, razão $U=M / S^{2}$ (c), em que $M$ é o número quadrático médio de recuperados e $S$ é o número médio de recuperados, e o produto entre as quantidades $U$ e $P(\mathrm{~d})$ versus a probabilidade de infecção $a$ para diferentes tamanhos de rede quadrada de lado $L$. As probabilidades de morte e nascimento de vetores são $e=0,200$ e $r=0,1000$, respectivamente. 


\subsection{Expoentes críticos estáticos}

Na seção 3.7, é demonstrada a relação entre o modelo SIR com a percolação usual (seção 2.6). Como neste modelo foram usados os mesmos processos de transição do modelo SIR (para os hospedeiros), é possível investigar o comportamento crítico deste modelo analisando as mesmas grandezas estacionárias estudadas para o modelo SIR.

Por meio da associação entre estes modelos, sabe-se que a probabilidade da epidemia atingir a borda da rede (parâmetro de ordem) e o cumulante, no ponto crítico, obedecem as seguintes formas de escala:

$$
P \sim L^{-\beta / \nu_{\perp}}
$$

$\mathrm{e}$

$$
U \sim L^{\beta / \nu_{\perp}}
$$

em que $\beta$ é o expoente crítico associado ao parâmetro de ordem e $\nu_{\perp}$ é o expoente crítico associado ao comprimento de correlação espacial.

Pela relação (5.19) pode-se escrever:

$$
\log \left(P_{L}\left(a_{c}\right)\right)=\frac{-\beta}{\nu_{\perp}} \log (L)+K,
$$

em que $K$ é uma constante. Utilizou-se essa relação e calculou-se a probabilidade $P$, no ponto crítico, para diferentes tamanhos de rede, com o intuito de se obter a razão entre $\beta$ e $\nu_{\perp}$. Na figura 5.9, consta $\log (P)$ versus $\log (L)$ para $a=0,33180$. Ajustando uma reta aos dados, obtem-se $\beta / \nu_{\perp}=0,105(2)$ coerente com o valor esperado para a classe de universalidade da percolação dinâmica 87].

Na figura 5.10, observa-se $\log (U)$ versus $\log (L)$ para $a_{c}=0,33180$ e, conforme esperado, o coeficiente da reta ajustada aos dados é $\beta / \nu_{\perp}=0,105(2)$. Devido a essas relações, o produto entre $U$ e $P$ no ponto crítico torna-se um valor universal, independente de $L$. Pelo gráfico de $U P$ versus $1 / L$ mostrado na figura 5.11, considerando somente tamanhos $L$ grandes e extrapolando para $L \rightarrow \infty$, obtem-se $U P_{c}=1,016(1)$, que está coerente com os valores críticos obtidos para o modelo de percolação usual [46].

É possível ainda explorar o comportamento crítico da distribuição estacionária de aglomerados para determinar o ponto crítico. Nas seções 3.7 e 3.7, observou-se que, para um conjunto grande de aglomerados, a probabilidade de haver um aglomerado de tamanho maior ou igual a $s$ sítios obedece a relação

$$
P_{\geq s} s^{\tau-2} \sim k_{1}+K_{2} \epsilon s^{\sigma}
$$




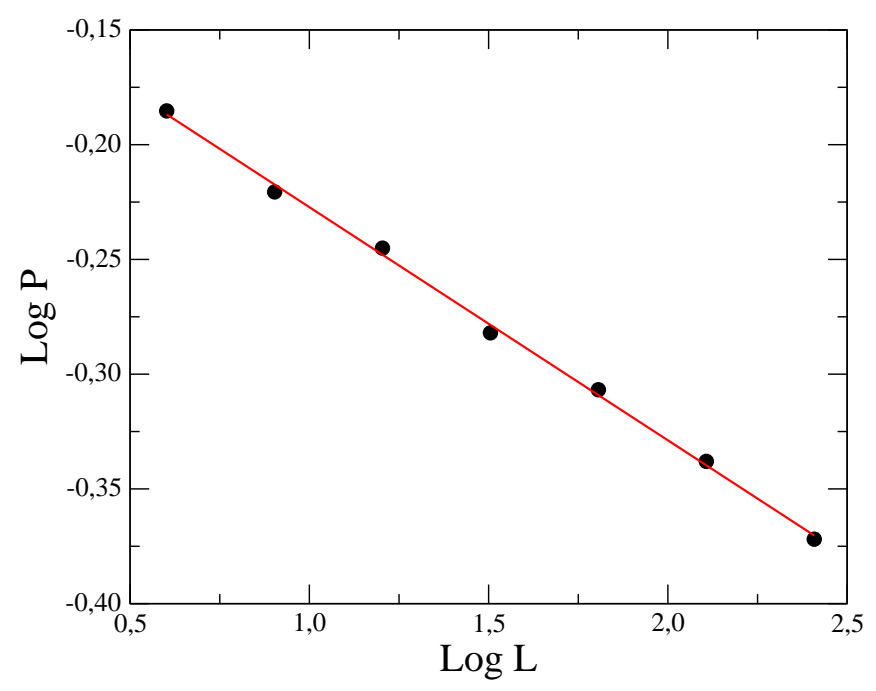

Figura 5.9: $\log (\mathrm{P})$ versus $\log (\mathrm{L})$. As linhas tracejadas são o ajuste linear aos dados.

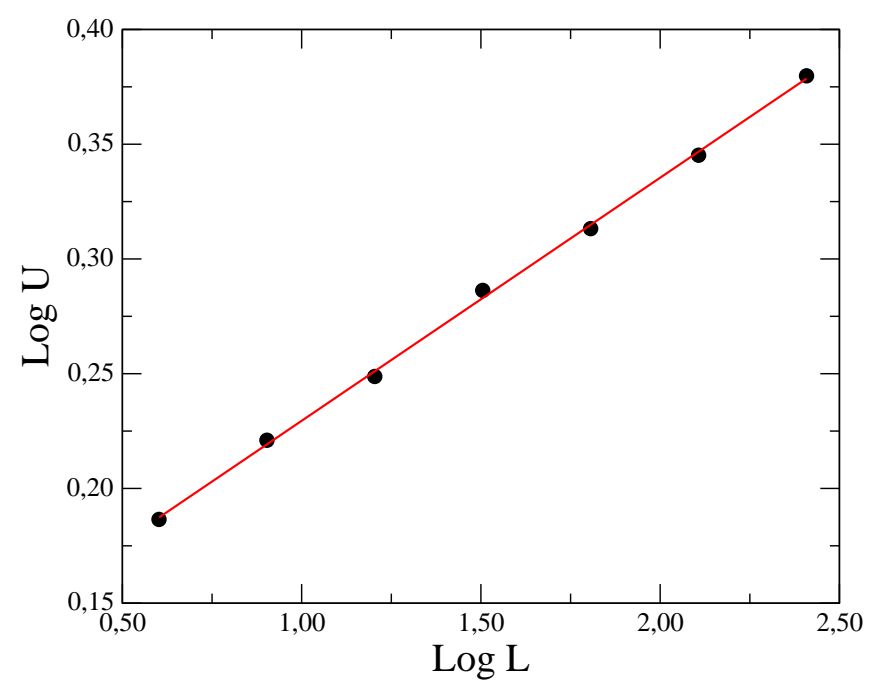

Figura 5.10: $\log (\mathrm{U})$ versus $\log (\mathrm{L})$.

em que $s$ é o tamanho do aglomerado, $K_{1}$ e $K_{2}$ são constantes, $\epsilon=a-a_{c}$, $\beta$ e $\tau$ são expoentes críticos que, em duas dimensões, são iguais à 187/91 e $36 / 91$, respectivamente.

Na figura 5.12 tem-se $P_{\geq s} s^{\tau-2}$ versus $s^{\sigma}$ para diferentes probabilidades a. Pela relação 5.22 no ponto crítico, $\epsilon=0$ e, então, $P_{\geq s} s^{\tau-2}$ é constante. Ajustando uma reta para os dados de cada probabilidade $a$, obtem-se os coeficientes lineares das retas e, pelo gráfico desses coeficientes versus a, 


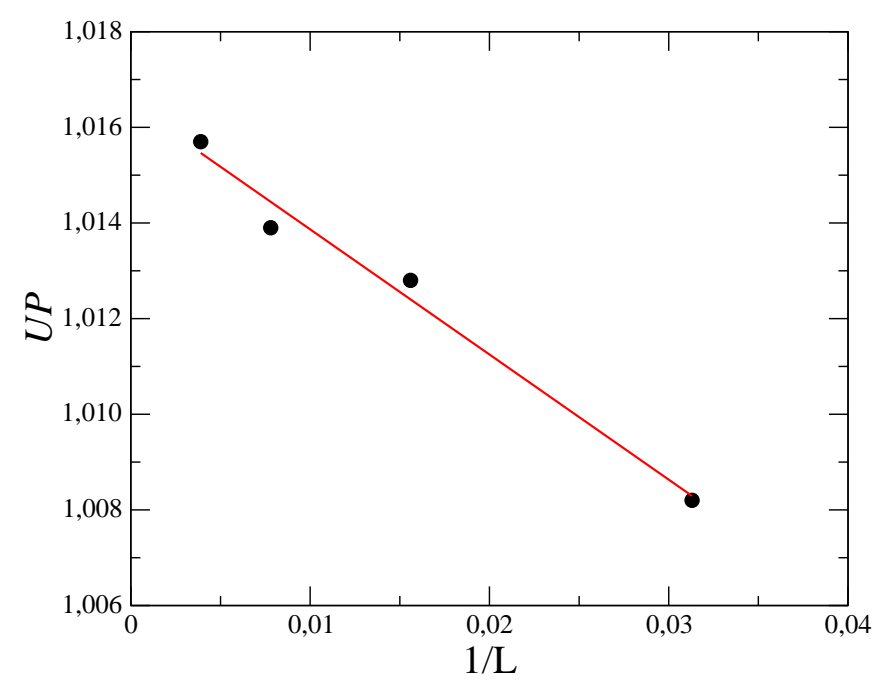

Figura 5.11: Grandeza UP versus 1/L.

pode-se calcular o valor de $a$ para o qual $P_{\geq s} s^{\tau-2}$ é constante. A partir da análise dos dados constantes na figura 5.13, obtem-se $a_{c}=0,331800(5)$.

Embora o número total de aglomerados seja pequeno (simulação com $75 \times 10^{3}$ amostras independentes), bem como o tamanho da rede $L=128$, verificou-se que este método permite calcular o ponto crítico com grande precisão.

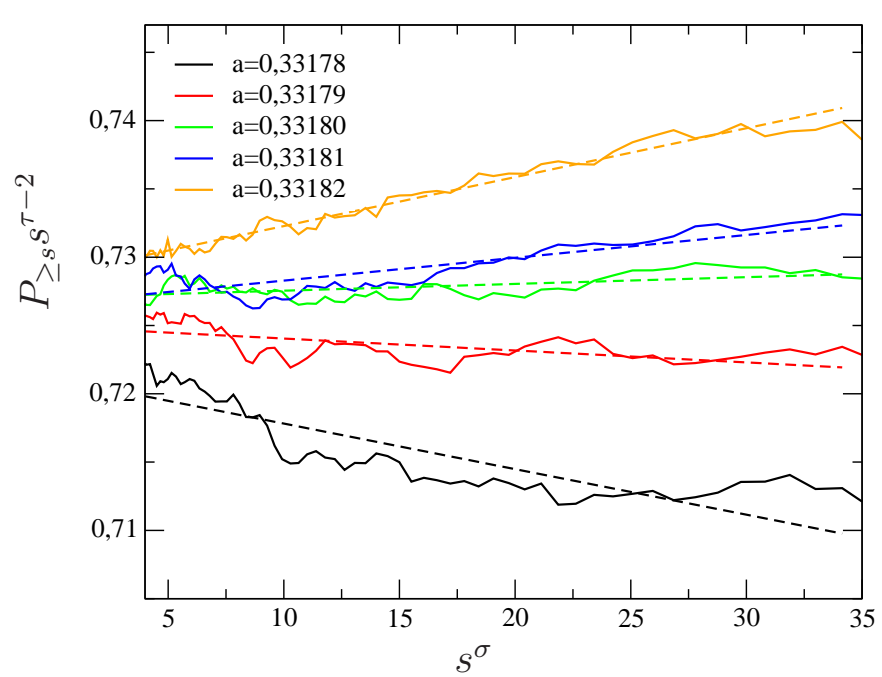

Figura 5.12: $P_{\geq s} s^{\tau-2}$ versus $s^{\sigma}$. 


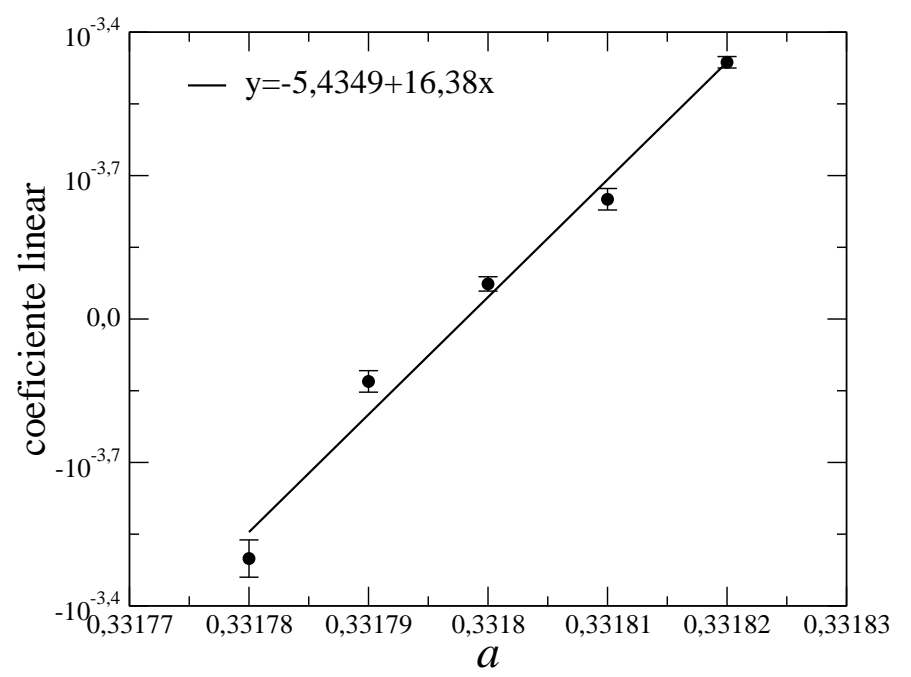

Figura 5.13: Coeficiente linear $K_{2}$ versus a para os dados da figura 5.12 .

\subsection{Simulações dependentes do tempo}

Uma forma computacionalmente eficiente de determinar os pontos críticos consiste em usar o método proposto por Grassberger e de la Torre [41, no qual se analisam a evolução temporal do número de infectados, a probabilidade de, em um dado instante, haver infectados e o raio quadrático médio de espalhamento da doença. Estas grandezas dependem fortemente da condição inicial e devem ser calculadas usando um conjunto de realizações que evoluem a partir de um estado que está na iminência do sistema cair em um estado absorvente. Usualmente, isso é feito colocando-se somente um indivíduo infectado no centro da rede, estando todos os demais indivíduos suscetíveis. Neste modelo, como há duas populações, tem-se arbitrariedade para escolher um infectado em uma das sub-redes. Para manter coerência com a condição inicial usada nas simulações estacionárias, impõe-se que a epidemia espalha sobre uma população com todos os indivíduos suscetíveis (vetores e hospedeiros) a partir de um único vetor infectado inserido no centro da rede.

Para realizar as simulações dependentes do tempo, utilizaram-se as mesmas regras locais apresentadass para algoritmo das simulações estacionárias, mas inseriu-se no passo $1 \mathrm{um}$ contador de tempo cujo incremento em uma unidade corresponde ao sorteio de $N$ sítios, escolhidos arbitrariamente. No passo 4 , foram calculados o número médio de vetores e hospedeiros infectados $N_{i}(t)$, a probabilidade de haver infectados em cada sub-rede $P_{s}(t)$ e o raio quadrático médio de espalhamento da epidemia em cada sub $=$ rede $R^{2}(t)$. Em 
cada realização, escolheu-se uma semente para o gerador de números pseudoaleatórios e deixou-se o sistema evoluir assincronamente, conforme as regras de interações locais, até o instante em que a epidemia cessou ou atingiu a borda da rede.

Na seção 4.6, viu-se que as relações para o número médio de infectados $N_{i}(t)$, a probabilidade de sobrevivência do processo epidêmico $P_{s}(t)$ e o raio quadrático médio de espalhamento da epidemia $R^{2}(t)$ no ponto crítico obedecem às seguintes leis de potência:

$$
\begin{gathered}
N_{i}(t) \sim t^{\eta}, \\
P_{s}(t) \sim t^{-\delta},
\end{gathered}
$$

$\mathrm{e}$

$$
R^{2}(t) \sim t^{z}
$$

em que $\eta, \delta$ e $z$ são os expoentes críticos dinâmicos associados ao número de indivíduos infectados, à probabilidade de sobrevivência da epidemia e ao raio quadrático médio, respectivamente. Próximo do ponto crítico, essas relações podem ser aproximadas usando as relações (4.64.4.66).

A partir dos resultados das simulações estacionárias, como demonstrados nas figuras 5.5,5.8, sabe-se aproximadamente qual é o limiar de infecção para cada probabilidade de morte dos vetores analisada. O conjunto de pontos críticos é parte da linha crítica associada à probabilidade de nascimento de vetores $r=0,1$, com a probabilidade de recuperação (imunização) dos hospedeiros fixada pela condição de normalização $c=1-r-2 a-2 e$.

Na figura 5.14 constam, em escala log-log, o número médio de vetores infectados $N_{i}(t)$, a probabilidade de sobrevivência $P_{s}(t)$ e o raio quadrático médio $R^{2}(t)$ em função do tempo para diferentes probabilidades de infecção $a$, com as probabilidades de nascimento e morte dos vetores fixadas em $r=0,1$ e $e=0,1$. Pela equação (5.23), como no ponto crítico $N_{i}(t)$ cresce com expoente $\eta$, acima do ponto crítico, o comportamento assintótico evidencia que o número de infectados supera a lei de potência e, abaixo do ponto crítico (no limite de tempo muito grande), o número de infectados tende a decrescer e ir para um valor nulo. Isto porque, embora a simulação seja feita em uma rede de tamanho finito, a análise de dados é feita supondo uma rede infinita e, neste caso, a epidemia poderia se espalhar por tempo indefinido (fase ativa $a>a_{c}$ ) ou cessar após um transiente de tempo (fase inativa $a<a_{c}$ ).

Para determinar o ponto crítico e os expoentes críticos, utilizaram-se as relações (4.64,4.66), cujo ajuste aos dados correspondentes a $N_{i}(t), P_{s}(t)$ e $R^{2}(t)$ são as inclinações locais, calculadas em intervalo $m=\Delta t=50$. Na figura 5.15 observam-se os expoentes dinâmicos $\eta(t)$ e $\delta(t)$ versus $1 / t$, que são as inclinações locais das curvas de $N_{i}(t)$ e $P_{s}(t)$ mostradas na figura 5.14 . 

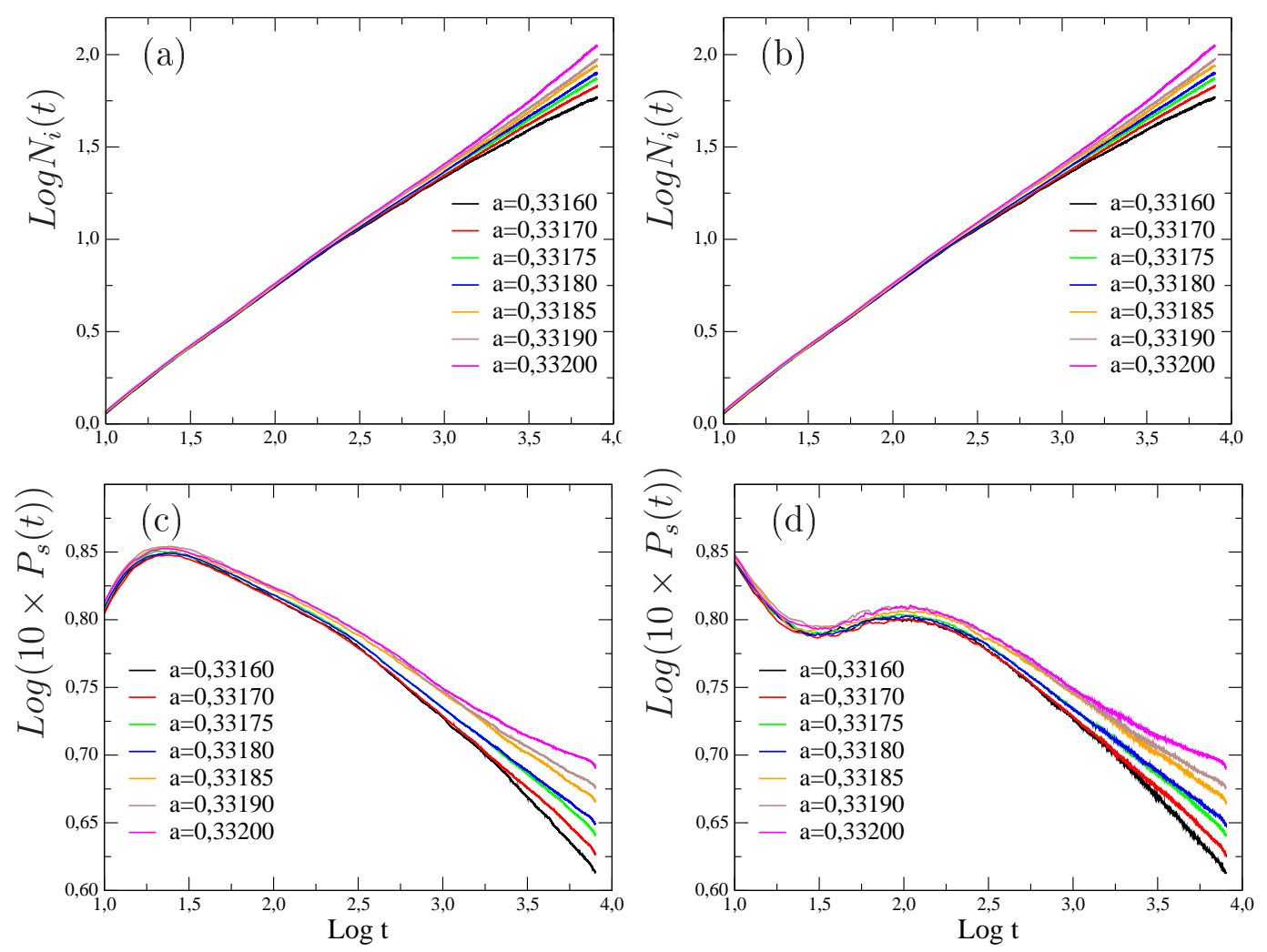

Figura 5.14: Em escala log-log: número de hospedeiros infectados (a) e vetores infectados (b) versus tempo e probabilidade de sobrevivência do processo epidêmico na subrede de hospedeiros (c) e de vetores (d).

Para $a=0,33180, \eta(t)$ tende a um valor constante, isto é, no limite $t \rightarrow \infty$ com $L$ muito grande, o expoente não depende do tempo. Para $a>a_{c} \mathrm{com}$ $1 / t$ tendendo a zero $\eta(t)$ cresce, e abaixo do limiar de infecção $\eta(t)$ decresce a medida que $1 / t$ vai a zero. Considerando estes dados, pode-se afirmar que $a_{c}=0,33180(1)$ o expoente dinâmico é $\eta=0,58(1)$, cujas incertezas estão relacionadas ao grau de distinção entre crescimento e decrescimento de $\eta(t)$ em função do tempo. Determinado o ponto crítico, calcularam-se os expoentes críticos $\delta$ e $z$ associados a $P_{s}(t)$ e $R^{2}(t)$. Analisando os dados de $P_{s}(t)$ e $R^{2}(t)$, obtiveram-se os expoentes críticos dinâmicos $\delta=0,092(1)$ e $z=1,69(5)$, que são os mesmos obtidos para o modelo SIR e o outro modelo de infecção discutido nos capítulos 3 e 4. Isso indica que este modelo também pertence à classe de universalidade da percolação dinâmica.

Para determinar outros pontos críticos, deve-se fixar as probabilidades de nascimento $r$ e morte $e$ dos vetores e variar a probabilidade de infecção, 

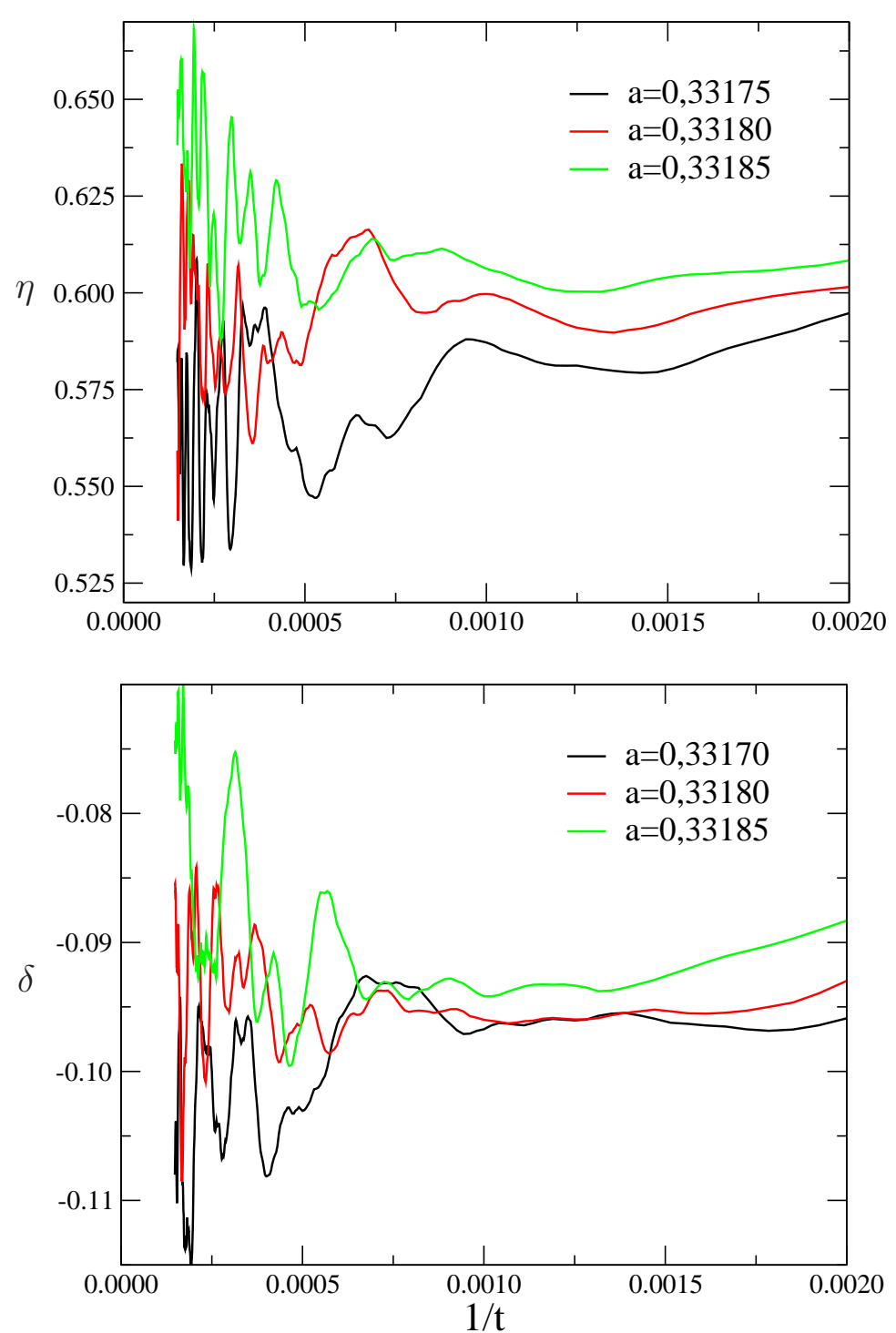

Figura 5.15: Expoentes dinâmico $\eta(t)$ e $\delta(t)$ versus $1 / t$ para diferentes probabilidades de infecção.

fazer simulações para cada conjunto de parâmetros e analisar as quantidades conforme o procedimento discutido anteriormente. Como se trata de repetir o mesmo método, apresentou-se somente o diagrama de fase. Na figura 5.16, é representado o diagrama de fase do modelo no plano a versus e para $r=0,1$. A linha tracejada é a linha crítica obtida por meio da aproximação de campo médio por pares e a linha contínua é a linha crítica obtida a partir de simulações de Monte Carlo (apenas os pontos críticos com barra de incerteza foram determinados por meio de simulações; a linha é uma curva ajustada 
a esses pontos e serve apenas de guia para os olhos). Na figura, a região abaixo de cada linha crítica corresponde à fase sem espalhamento da epidemia. Quando a probabilidade de infecção $a$ torna-se maior que o valor crítico $a_{c}$, a epidemia se espalha formando um aglomerado (no estado estacionário) de hospedeiros recuperados. No diagrama de fase, verifica-se que para $e=0$ o limiar de infecção é não-nulo (na aproximação de campo médio por pares e simulações de Monte Carlo) e, à medida que $e \rightarrow 0,5-(c+r) / 2$, a solução de campo médio por pares concorda qualitativamente com os resultados obtidos por meio de simulações dependentes do tempo. Isso não ocorre com a aproximação de campo médio simples para a qual, quando $e \rightarrow 0$ a probabilidade de infecção $a \rightarrow 0$.

Embora todos os resultados apresentados neste trabalho sejam referentes a $r=0,1$, é possível extrapolar o comportamento crítico visto no diagrama de fase para outros valores de $r$. Para $r$ próximo de zero, a linha crítica deve se aproximar de $c=0$ quando $e \rightarrow 0,5$, provocando um aumento da fase sem espalhamento da epidemia. Para $r>0,1$, os vetores nascem com probabilidade maior e, consequentemente, a fase com espalhamento da epidemia deve crescer em relação à fase vista para $r=0,1$.

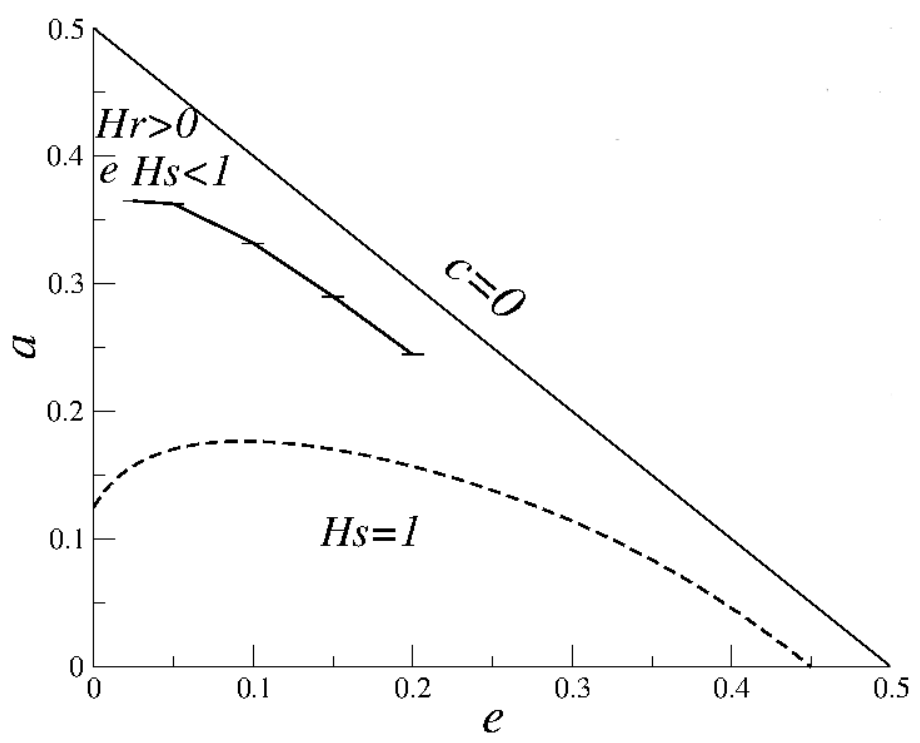

Figura 5.16: Diagrama de fase do modelo no plano a versus e, com a linha crítica determinada via campo médio por pares (linha tracejada) e simulações numéricas do modelo. Nos dados obtidos por meio de simulações de Monte Carlo a incerteza da probabilidade de infecção é da mesma ordem de grandeza da espessura da linha. 


\section{Capítulo 6}

\section{Comparações e aplicações dos modelos}

Os modelos discutidos nos capítulos 4 e 5, denominados modelos I e II, surgiram a partir de tentativas de formular um modelo capaz de descrever as séries temporais (número de casos notificados na rede de saúde pública em função do tempo) para algumas epidemias de dengue em área urbana 75-79, 85, 89 92. E, além disso, investigar qual é o efeito do combate aos mosquitos (fase adulta do vetor no caso do modelo I e nas fases larval e adulta no caso do modelo II) no controle das epidemias.

Por esse ponto de vista, nesses modelos, deve-se considerar a trasmissão devida a somente um sorotipo do vírus da dengue, sendo a fêmea do mosquito Aedes aegypti o vetor e o homem o hospedeiro.

A probabilidade de infecção $a$ está associada ao número de picadas por pessoa por unidade de tempo. A probabilidade $c$ é proporcional ao inverso do tempo de viremia (intervalo de tempo que o homem infectado pode transmitir o vírus). E a probabilidade $e$ representa a probabilidade de morte natural do mosquito e que também pode acrescentar a morte devido ao uso de inseticidas que são aplicadas pelo centro de controle de zoonoses.

No modelo II, considerou-se o nascimento de mosquitos com probabilidade $r$, a qual está associada à capacidade de reprodução dos mosquitos e, embora alguns autores usem esse parâmetro como sendo dependente das condições climáticas, variando sazonalmente, manteve-se a probabilidade $r$ constante no tempo. Este modelo proposto permite investigar a transmissão da doença com outros valores de $r$, mas, devido ao desconhecimento acerca de um parâmetro confiável e dificuldade de variar $r$ em todo seu domínio, fixou-se $r=0,100 \mathrm{em}$ todas as análises do modelo. Essa probabilidade reflete diretamente a concentração de mosquitos no ambiente. 
$\mathrm{Na}$ seção 6.1, serão comparados os resultados de campo médio e simulações dos modelos. Procurar-se-á também evidenciar as semelhanças e diferenças entre eles. Na seção 6.2, o modelo I será usado para descrever uma epidemia de dengue.

\subsection{Comparação entre os modelos}

Analisando as figuras 6.1 e 6.1, que mostram as regras locais de cada um dos modelos, observa-se que os processos de infecção e recuperação seguem as mesmas regras para ambos os modelos. No modelo I, considerou-se que a concentração de vetores no ambiente é grande e, assim, cada unidade espacial está sempre ocupada por um mosquito suscetível ou infectado. Entretanto, no modelo II, supõe-se que a morte de um vetor dá origem a um espaço vazio e, nesse caso, o nascimento de um vetor não é imediato e, devido à capacidade de suporte do meio, só pode ocorrer em um espaço vazio. A probabilidade de nascimento dos mosquitos $r$ pode representar a probabilidade natural de sobrevivência dos mosquitos nos primeiros estágios de vida (ovos e larvas) até a fase adulta (momento que a fêmea necessita de sangue para maturar os ovos). Em caso de controle de mosquitos na fase larval, essa probabilidade deve ser menor que a probabilidade natural de sobrevivência.
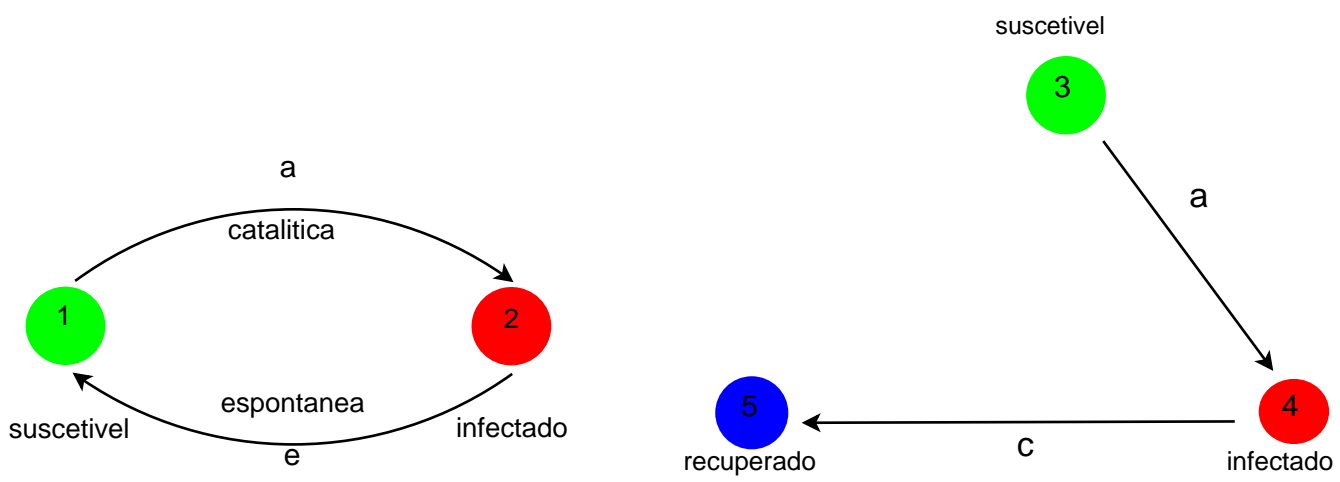

Figura 6.1: Regras de transições locais para o modelo I. Somente o processo de infecção cruzada é catalítico; as demais transições são espontâneas. Para denotar o estado de saúde do indivíduo com relação a uma certa doença associa-se uma variável estocástica $\eta$ que assume os valores 1 ou 2 para denotar vetor suscetível ou infectado; e 3,4 ou 5 para representar um hospedeiro suscetível, infectado ou recuperado.

Usando atualização assíncrona, ao sortear qualquer sítio de um reticulado, deve-se aplicar um dos processos de transições locais. Desse modo, a soma 

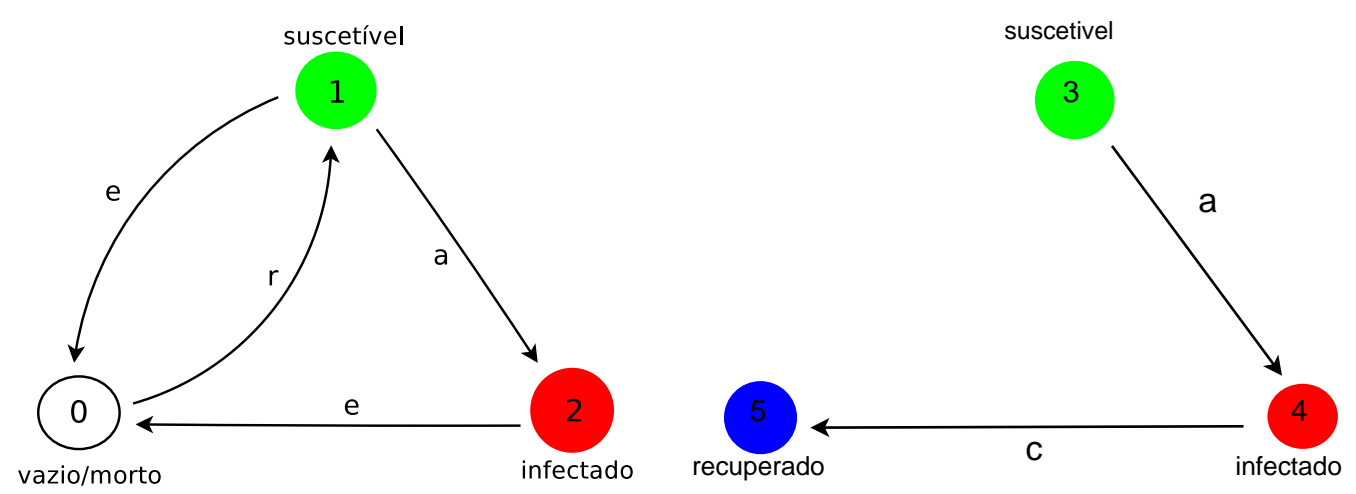

Figura 6.2: Regras de transições locais para o modelo II. Somente o processo de infecção cruzada é catalítico; as demais transições são espontâneas. Para denotar o estado de saúde do indivíduo com relação a uma certa doença associa-se uma variável estocástica $\eta$ que assume os valores 0,1 ou 2 para denotar a ausência de vetor, a presença de um vetor suscetível ou infectado; e 3,4 ou 5 para representar um hospedeiro suscetível, infectado ou recuperado

de possibilidade de eventos deve ser unitária, que pode ser parametrizada da seguinte forma:

$$
e+2 a+c=1
$$

e

$$
r+2 e+2 a+c=1,
$$

para os modelos I e II, respectivamente.

Para analisar as similaridades entre os modelos, é necessário comparar as equações de evolução das densidades. Na tabela 6.1 são descritas as equações de evolução das densidades obtidas a partir da equação mestra para os dois modelos espacialmente estruturados.

Pela equação de evolução de $P(0)$ no modelo II mostrada na tabela 6.1, observa-se que, no regime estacionário, a densidade de sítios vazios é $P(0)=w^{*}=r /(r+e)$ e, quando a densidade de mosquitos infectados $P(2)=y \rightarrow 0$, a densidade de mosquitos suscetíveis torna-se $x^{*}=e /(r+e)$. Para comparar os dois modelos, uma vez que no segundo a sub-rede de mosquitos não está completamente preenchida por mosquitos, pois há espaços vazios, deve-se comparar a densidade de mosquitos suscetíveis do modelo I $x^{\prime}=1$ com a densidade $x^{*}=e /(r+e)$ do modelo II. Impondo um fator de proporcionalidade $\beta$ entre essas densidades obtemos:

$$
\beta e /(e+r)=1
$$

$\mathrm{Ou}$

$$
\beta e=e+r .
$$


Tabela 6.1: Comparação entre as equações para as densidades de indivíduos para os dois modelos

\begin{tabular}{c|c}
\hline Modelo I & Modelo II \\
\hline$\frac{d P(1)}{d t}=e P(2)-a P(14)$ & $\frac{d P(0)}{d t}=e[P(1)+P(2)]-r P(0)$ \\
$\frac{d P(2)}{d t}=a P(14)-e P(2)$ & $\frac{d P(2)}{d t}=a P(14)-e P(2)$ \\
$\frac{d P(3)}{d t}=-a P(32)$ & $\frac{d P(3)}{d t}=-a P(32)$ \\
$\frac{d P(4)}{d t}=a P(32)-c P(4)$ & $\frac{d P(4)}{d t}=a P(32)-c P(4)$ \\
\hline
\end{tabular}

Para evitar confusão entre as probabilidades de morte de um modelo e outro, será denominada como $E$ a probabilidade de morte para o modelo I, diferenciando-a da probabilidade de morte $e$ do modelo II. Pelas equações (6.1) e (6.2), tem-se: $r+2 e+2 a+c=1$ e $E+2 a+c=1$. E, a partir delas, pode-se usar que a probabilidade de nascimento de mosquitos é dada pela expressão:

$$
\begin{aligned}
r+2 e & =E \\
\beta e+e & =E \\
E & =e \frac{e+2 r}{r},
\end{aligned}
$$

que permite comparar a probabilidade de morte do modelo I $E$ à probabilidade de morte $e$ do modelo II.

Na figura 6.3, consta o diagrama de fase dos dois modelos, com as curvas de transição de fase determinadas por meio de aproximações de campo médio com pares e simulações computacionais dos modelos. Abaixo de cada curva, tem-se a fase sem espalhamento da doença e a parte superior corresponde à fase com espalhamento da doença. Neste diagrama a probabilidade de morte dos mosquitos $e$ para o segundo modelo não está reescalada para ser equivalente à probabilidade de morte do modelo $\mathrm{I}$.

Usando a equação (6.5) para reescalar a probabilidade de morte dos mosquitos para o modelo II, essa se torna compatível com a probabilidade usada no modelo I. E, assim, obtem-se o diagrama de fase mostrado na 
figura 6.4. Esse diagrama apresenta ainda alguns problemas, por exemplo, no modelo II, a probabilidade de infecção não se anula quando $E \rightarrow 1$. Entretanto, quando $e / r$ é pequeno os dois modelos descrevem linhas de transição de fase com o mesmo comportamento.

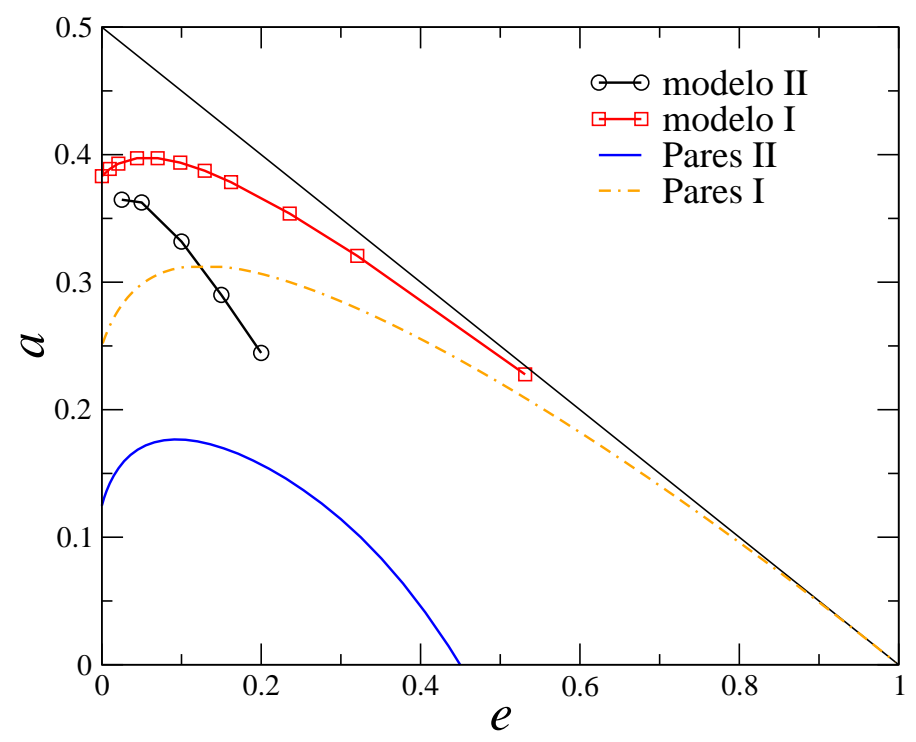

Figura 6.3: Diagrama de fase para os modelos I e II, no plano a versus e, segundo as aproximações de campo médio por pares e simulações computacionais.

Ao fazer o diagrama de fase em termos das probabilidades relativas $e / c$ versus $a / c$, reescalando a probabilidade de morte dos mosquitos (usando a equação (6.5) ), obtem-se o diagrama apresentado na figura 6.5 no qual podemos ver que, se analisadas as linhas críticas em termos de probabilidades relativas à probabilidade de recuperação, os dois modelos apresentam diagramas de fase muito semelhantes. Além disso, verifica-se que a fase com espalhamento da epidemia (fase ativa) para o modelo II é menor (no limite $r<<e$ ) em relação à fase com espalhamento obtida para o modelo I. Portanto, pode-se concluir que o controle de natalidade de vetores, associado ao controle da população na fase adulta é muito mais eficiente para impedir o espalhamento da epidemia, que controlar somente a mortalidade de vetores adultos. 


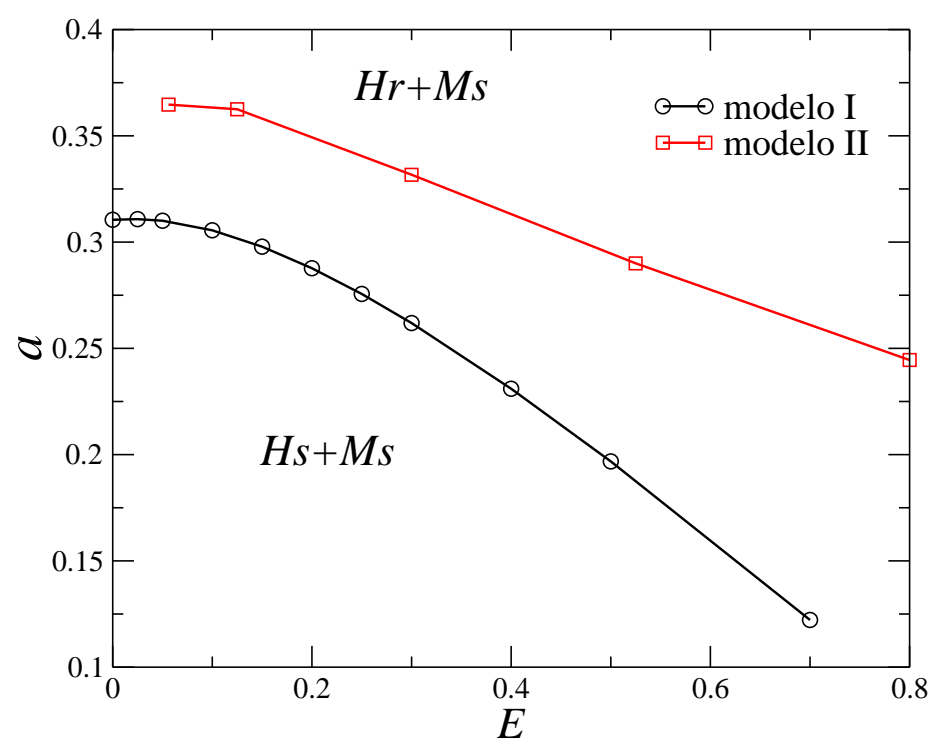

Figura 6.4: Diagrama de fase para os modelos I e II, no plano a versus $E$ ( $a$ e $c$ não reescalados). A probabilidade de morte de mosquitos $e$ para o segundo modelo foi reescalada por meio da equação 6.5. Os dados exibidos nessa figura foram obtidos por meio de simulações de Monte Carlo.

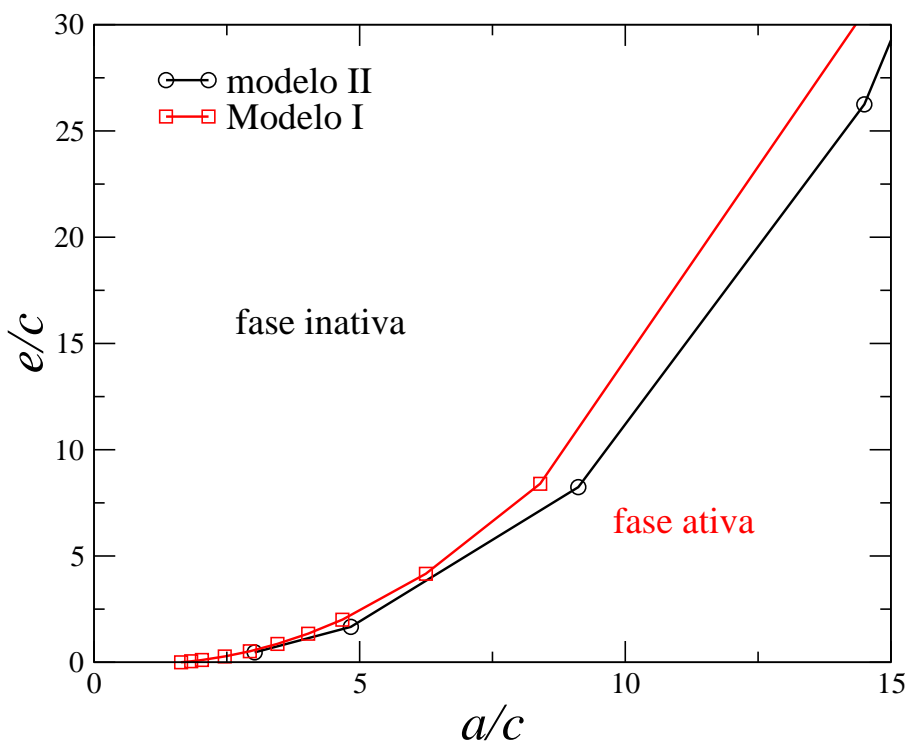

Figura 6.5: Diagrama de fase para os modelos I e II, no plano e/c versus $a / c$. A probabilidade de morte de mosquitos e para o segundo modelo foi reescalada por meio da equação 6.5. 
Pode-se ver que o diagrama de fase em termos das probabilidades relativas $e / c$ e $a / c$ favorece a visualização da dependência do limiar de infecção com o controle vetorial. No diagrama de fase no plano a versus e essa dependência é confusa por não ficar óbvio que o aumento da probabilidade de morte dos mosquitos (quando e é pequeno) de fato causa redução no limiar de infecção.

É possível melhorar essa compreensão do diagrama de fase fazendo algumas adaptações. Seja a razão $e / c$ igual a uma constante $\alpha$. Como no modelo I aplicou-se a condição de normalização $2 a+c+e=1$, pode-se reescrever a fração da seguinte forma:

$$
\begin{aligned}
\frac{e}{c} & =\alpha \\
\frac{e}{1-2 a-e} & =\alpha \\
e+e \alpha & =\alpha-2 \alpha a \\
a & =\frac{1}{2}-e\left(\frac{1+\alpha}{2 \alpha}\right) .
\end{aligned}
$$

Esta equação significa que, aumentando a probabilidade de morte dos mosquitos por um fator $\alpha$, relativo à probabilidade de recuperação dos homens, reduz-se o limiar de infecção conforme a equação (6.6). Apenas como exemplo, usando $\alpha=2$, obtem-se a linha de controle vetorial (linha pontilhada) descrita na figura 6.6, que indica a redução da probabilidade de infecção $a$ em função do aumento da probabilidade de morte $e$. Como esse diagrama de fase constam as linhas críticas obtidas por meio de campo médio simples, por pares e por de simulações de Monte Carlo, deve-se lembrar que na fase II (fase abaixo de cada linha crítica) não há epidemia e, portanto, o controle vetorial é desnecessário para fins de controle de transmissão da doença. Na fase I há espalhamento da doença e o estado estacionário corresponde à situação em que a população de homens fica constituída por indivíduos suscetíveis e recuperados e a população dos mosquitos, somente por mosquitos suscetíveis $\left(H_{r}>0\right.$ e $\left.H_{s}<1\right)$. Na fase II, a doença cessa em tempo muito curto, sem haver espalhamento da doença e, assim, o estado absorvente corresponde a ambas as populações suscetíveis. 


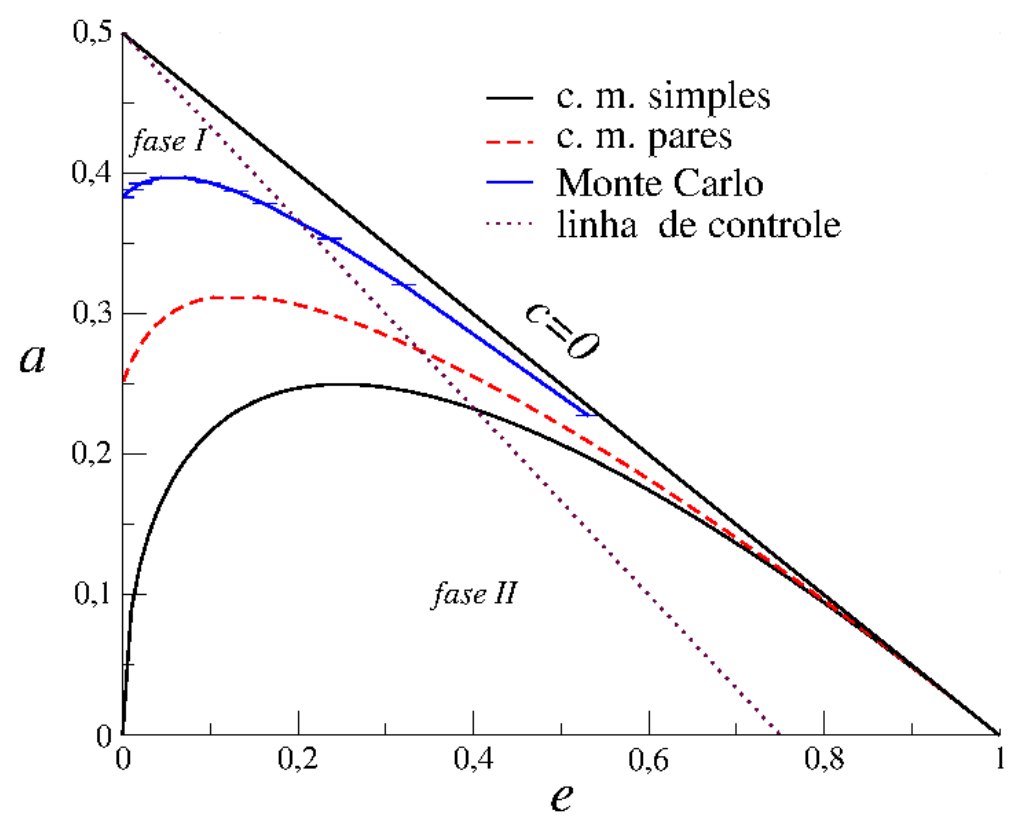

Figura 6.6: Diagrama de fase para o modelo I, com a linha crítica determinada via campo médio simples (curva contínua inferior), por pares (curva tracejada) e simulações de Monte Carlo (curva superior com barras de incertezas para os pontos críticos). A linha pontilhada é a redução da probabilidade de transmissão da doença caso haja controle da população de mosquitos. 


\subsection{Aplicação do modelo I}

Usando as equações obtidas por meio da aproximação de campo médio por pares para o modelo I, dadas pelas equações (4.33)-(4.37), foi analisada a epidemia de dengue registrada na cidade de Salvador-Brasil no ano de 1995, descrita na referência [89. Ainda que a solução exata do sistema de equações (4.33)-(4.37) não tenha sido determinada neste trabalho, pode-se usar parâmetros adequados para obter a evolução temporal do número de indivíduos infectados e comparar com a série epidemica real.

A partir da distribuição temporal de casos de dengue notificados para a epidemia de dengue descrita na referência [89] e usando os parâmetros do modelo $a=2, e=0,2$ e $c=9,8$, em que $a$, e e $c$ devem ser entendidos como taxas de infecção por dia, taxa de morte por dia e taxa de recuperação por dia, respectivamente. Esse valores baseiam se nos parâmetros descritos na referência 93. Supondo uma população exposta (em contato direto com os mosquitos) $N=3,2 \times 10^{4}$, a qual multiplicada pela densidade de infectados resulta o número de infectados, e integrando o sistema de equações (4.33)(4.37) com uma densidade inicial de vetores infectados igual a $10^{-6}$ obtemos a curva epidemica mostrada na figura 6.7. Nessa figura pode-se ver que o modelo I consegue descrever qualitativamente e quantitativamente a série temporal em todo o intervalo de duração da epidemia. Por não ter obtido a solução exata não se aplicou a minimização do desvio da curva epidemica em relação aos dados reais. 


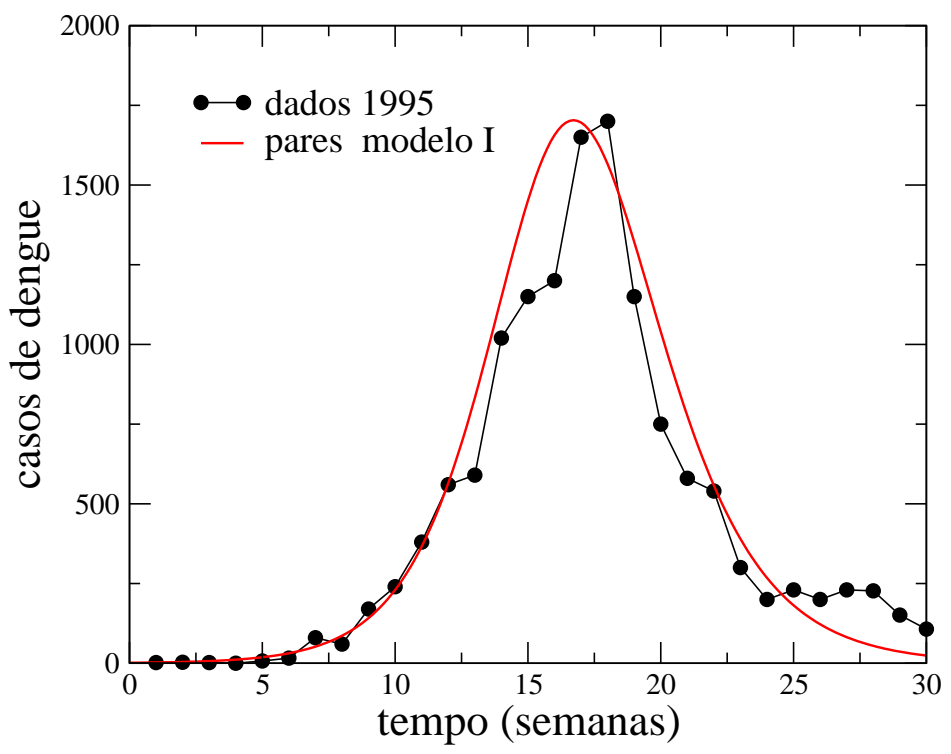

Figura 6.7: Número de casos de dengue registrados na cidade de Salvador em 1995. A linha vermelha é o número de infectados obtidos por meio da aproximação de campo médio por pares para o modelo I, usando os parâmetros $a=2, e=0,2, c=9,8$. 


\section{Capítulo 7}

\section{Conclusões}

Nesta pesquisa investigou-se o modelo SIR estocástico definido em redes hipercúbicas por meio de simulações de Monte Carlo e também por meio de aproximações de campo médio dinâmico. As aproximações de campo médio mostraram que o modelo exibe um ponto crítico que separa uma fase em que há espalhamento da epidemia e outra em que não há espalhamento da epidemia [34]. Essas aproximações, principalmente a de pares (que leva em conta correlações de dois sítios), fornecem resultados qualitativamente compatíveis aos resultados obtidos neste estudo por meio de simulações de Monte Carlo.

As investigações por meio de simulações de Monte Carlo [34, 46], permitiram determinar o ponto crítico do modelo SIR e obter os expoentes críticos. Para tanto, estabeleceu-se em primeiro lugar a correspondencia entre grandezas relevantes para a caracterização do comportamento crítico do modelo SIR e grandezas consideradas no modelo de percolação usual. Em seguida, realizou-se uma análise de escala finita dos dados obtidos por meio de simulações de Monte Carlo para o modelo SIR definido em redes quadradas e triangulares. Dessa maneira, obtiveram-se expoentes críticos estáticos que são consistentes com os da classe de universalidade da percolação dinâmica isotrópica. Para tanto, utilizaram-se simulações dependentes do tempo [51].

Além disso, definiu-se o parâmetro de ordem adequado para a transição no modelo SIR e introduziu-se uma nova função universal para o modelo SIR e para modelos de percolação isotrópica [46]. Essa grandeza foi denominada $U P$, na qual $P$ denota o parâmetro de ordem (para o modelo SIR) e a grandeza $U$ representa a razão entre o valor médio do quadrado do número de sítios no estado $R$ (ocupado por um indivíduo recuperado) e o quadrado do número médio de sítios no estado $R$. No ponto crítico, $U P$ não depende do tamanho do sistema e, portanto, a partir da análise dessa grandeza como função do parâmetro de controle $c$ (probabilidade de recuperação para o 
modelo SIR) para diferentes tamanhos do sistema, pode-se obter o ponto crítico.

A partir do modelo de contato, do modelo SIR e do modelos SIRS, propuseram-se dois diferentes modelos epidêmicos nos quais o processo de transmissão é mediado por um vetor. Esses modelos foram denominados modelo I e modelo II. Foram consideradas duas populações formadas por hospedeiros e vetores que interagem entre si, transmitindo a doença de uma população para outra. O processso de infecção ocorre devido ao contato de um hospedeiro ou vetor suscetível com um vetor ou hospedeiro infectado. No modelo I, supõe-se que os vetores estão homogeneamente distribuídos pelo espaço e o intervalo necessário para o vetor nascer e atingir a fase adulta é muito pequeno comparado ao seu tempo de vida, podendo ser desprezado. Neste caso, cada vetor pode ser classificado como suscetível (S) ou infectado (I). Os hospedeiros podem ser divididos em três classes: suscetível(S), infectado (I) ou recuperado (R). No modelo II, tratou-se explicitamente o processo de nascimento e morte de vetores, adicionando um estado vazio (que representa a ausência de vetores) em que pode haver nascimento de um vetor suscetível. Estes modelos são definidos em uma rede quadrada bipartida em duas sub-redes A e B em que cada sítio da sub-rede A(B) pode ser ocupado por um único vetor(hospedeiro). As regras destes modelos são tais que, para o modelo I, as transições locais para os vetores sejam similares ao processo de contato e, no modelo II, às transições locais para os vetores sejam similares as transições do modelo SIRS. Em ambos, as transições locais envolvendo hospedeiros são análogas às regras do modelo SIR, com o processo de infecção proporcional ao número de vizinhos (da outra população) infectados. Estes modelos estocásticos foram investigados por meio de aproximações de campo médio e simulações de Monte Carlo. Verificou-se que estes modelos exibem transições de fase de $2^{a}$ ordem, em que a linha crítica separa uma fase em que a doença não se espalha e outra fase em que ocorre epidemia. Analisando as mesmas grandezas consideradas no modelo SIR, investigou-se o comportamento crítico destes modelos por meio de simulações de Monte Carlo. Os expoentes críticos obtidos mostram que ambos os modelos pertencem à classe de universalidade da percolação dinâmica isotrópica.

Os modelos discutidos neste trabalho são irreversíveis com muitos estados absorventes e, por meio destes modelos, são apresentadas diferentes abordagens sobre o espalhamento de epidemias para populações espacialmente estruturadas (isso é de grande importância para descrever epidemias reais). As investigações permitiram determinar a classe de universalidade dos modelos analisados e aprimorar métodos para estudar o comportamento crítico de modelos com muitos estados absorventes. É necessário aperfeiçoar estes modelos, inserindo diferentes classes de indivíduos suscetíveis (os quais 
podem tornar-se infectados com diferentes taxas), com o intuito de descrever de forma mais realista alguns tipos de epidemias cuja transmissibilidade está associada ao estado de imunidade de cada indivíduo. Além disso, para tratar a mobilidade dos indivíduos, pretende-se investigar os efeitos de difusão local dos indivíduos através da rede. Acredita-se que o processo de difusão é essencial para descrever a formação de múltiplos focos epidêmicos que favorecem o espalhamento da epidemia e dificultam o controle e erradicação de doenças infectocontagiosas. 


\section{Referências Bibliográficas}

[1] E. Renshaw, Modelling Biological Populations in Space and Time. (Cambridge University Press, Cambridge, 1995).

[2] H. W. Hethcote, "The mathematics of infectious diseases," SIAM J. Appl. Math., vol. 42, pp. 599-653, 2000.

[3] J. D. Murray, Mathematical Biology. (Springer-Verlag, New York, 1980).

[4] N. S. Goel and N. Richter-Dyn, Stochastic Models in Biology. (Academic Press, New york, 1974).

[5] N. Stollenwerk and V. A. A. Jansen, Criticality in Epidemiology, in Complex Population Dynamics: Nonlinear Modeling in Ecology, Epidemiology and Genetics. (World Scientific Lecture Notes in Complex Systems, Oldenburg, Potsdam and Tel Aviv, 2007).

[6] D. Mollison, The Structure of Epidemic Models. (Cambridge University Press, Cambridge, 1995).

[7] M. J. Keeling and P. Rohani, Modeling Infectious Diseases in Humans and Animals. (Princeton University Press, Princeton and Oxford, 2008).

[8] N. C. Grassly and C. Fraser, "Mathematical models of infectious disease transmission," Nat. Rev. Micro., vol. 6, pp. 477-487, 2008.

[9] R. M. Anderson and R. M. May, Infectious Diseases of Humans Dynamics and Control. (Oxford Science Publishing, London, 2008).

[10] N. T. J. Bailey, "The total size of a general stochastic epidemic," Biometrika, vol. 40, no. 1-2, pp. 177-185, 1953.

[11] N. T. J. Bailey, The Mathematical Theory of Epidemic. (Hahner, New York, 1957). 
[12] N. G. van Kampen, Stochastic Processes in Physics and Chemistry. (North-Holland, Amsterdam, 1992).

[13] N. Vandewalle and M. Ausloos, "Exact solution of the dynamic epidemic model on the bethe lattice," Phys. A, no. 230, pp. 1-10, 1996.

[14] R. M. Anderson and R. M. May, "Population biology of infectious diseases: Part i," Nature, vol. 280, pp. 361-367, 1979.

[15] W. O. Kermack and A. G. McKendrick, "A contribution to the mathematical theory of epidemics," Proc. R. Soc. Lond. A, vol. 115, pp. 700$721,1927$.

[16] H. J. Wearing, P. Rohani, and M. J. Keeling, "Appropriate models for the management of infectious diseases," PLoS Med., vol. 2, pp. 1-7, 2005 .

[17] R. M. Anderson and R. M. May, "Population biology of infectious diseases: Part ii," Nature, vol. 280, pp. 455-461, 1979.

[18] H. Nishiura, S. O. Brockmann, and M. Eichner, "Extracting key information from historical data to quantify the transmission dynamics of smallpox," Theor. Biol. Med. Mod., vol. 5, pp. 1-12, 2008.

[19] N. Stollenwerk and V. A. A. Jansen, "Meningitis, pathogenicity near criticality:the epidemiology of meningococcal disease as a model for accidental pathogens," J. Theor. Biol., vol. 222, pp. 347-359, 2003.

[20] S. M. Dammer and H. Hinrichsen, "Epidemic spreading with immunization and mutations," Phys. Rev. E, vol. 68, p. 016114, 2003.

[21] C. J. Rhodes and R. M. Anderson, "Epidemic thresholds and vaccination in a lattice model of disease spread," Theor. Pop. Biol., vol. 52, pp. 101$118,1997$.

[22] D. Bernoulli and S. Blower, "An attempt at a new analysis of the mortality caused by smallpox and of the advantages of inoculation to prevent it," Rev. Med. Virol., vol. 14, pp. 275-288, 2004.

[23] A. L. Lloyd and R. M. May, "Epidemiology: How Viruses Spread Among Computers and People," Science, vol. 292, pp. 1316-1317, 2001.

[24] N. Bahi-Jaber and D. Pontier, "Modeling transmission of directly trasmitted infectious diseases using colored stochastic petri nets," vol. 185, pp. 1-13, 2003. 
[25] R. M. Anderson and R. M. May, "Vaccination and herd immunity to infectious diseases," Nature, vol. 318, pp. 323-329, 1985.

[26] S. Gonçalves, M. Kuperman, and M. F. C. Gomes, "A social model for the evolution of sexually transmitted diseases," Phys. A, vol. 342, pp. $256-262,2004$.

[27] M. Amaku, F. A. B. Coutinho, R. S. Azevedo, M. N. Burattini, L. F. Lopez, and E. Massad, "Vaccination against rubella: Analysis of the temporal evolution of the age-dependent force of infection and the effects of different contact patterns," Phys. Rev. E, vol. 67, p. 051907, 2003.

[28] C. E. Mills, J. M. Robins, and M. Lipsitch, "Transmissibility of 1918 pandemic influenza," Nature, vol. 16, pp. 904-906, 2004.

[29] F. Brauer and P. van den Driessche, "Models for transmission of disease with immigration of infectives," Math. Biosci., vol. 17, pp. 143-154, 2001.

[30] F. Zhang and X. Zhao, "A periodic epidemic model in a patchy environment," J. Math. Anal. Appl., vol. 325, pp. 496-516, 2007.

[31] T. Tomé, Irreversibilidade. Modelos de rede com dinâmicas estocásticas. (Tese de Livre-Docência, Instituto de Física da Universidade de São Paulo, São Paulo, 1996).

[32] T. Tomé and M. J. de Oliveira, Dinâmica Estocástica e Irreversibilidade. (Edusp, São Paulo, 2001).

[33] J. Marro and R. Dickman, Nonequilibrium Phase Transitions in Lattice Models. (Cambridge University Press, Cambridge, 1999).

[34] D. R. de Souza and T. Tomé, "Stochastic lattice gas model describing the dynamics of the sirs epidemic process," Physica A, vol. 389, pp. 11421150, 2010.

[35] S. Davis, P. Trapman, H. Leirs, M. Begon, and J. A. P. Heesterbeek, "The abundance threshold for plague as a critical percolation phenomenon," Nature, vol. 454, pp. 634-637, 2008.

[36] M. J. Keeling, "The effects of local spatial structure on epidemiological invasions," Proceedings of the Royal Society of London. Series B: Biological Sciences, vol. 266, no. 1421, pp. 859-867, 1999. 
[37] V. Privman, Finite Size Scaling and Numerical Simulation of Statistical Systems. (World Scientific, Singapore, 1998).

[38] L. Landau and E. Lifshitz, Physique Statistique, vol. I. (MIR, Moscou, 1984).

[39] H. Hinrichsen, "Non-equilibrium critical phenomena and phase transitions into absorging states," Adv. in Phys., vol. 49, pp. 815-958, 2000.

[40] K. Binder and D. W. Heermann, Monte Carlo Simulation in Statistical Physics: An Introduction. (Springer-Verlag, Berlin, 1992).

[41] P. Grassberger, "On the critical-behavior of the general epidemic process and dynamical percolation," Math. Biosci., vol. 63, pp. 157-173, 1983.

[42] J. L. Cardy and P. Grassberger, "Epidemic models and percolation," J. Phys. A, vol. 18, pp. L267-L271, 1985.

[43] F. M. Neri, F. J. Pérez-Reche, S. N. Taraskin, and C. A. Gilligan, "Heterogeneity in susceptible-infected-removed (sir) epidemics on lattices," J. R. Soc. Interface, vol. 8, no. 55, pp. 201-209, 2011.

[44] T. Tomé and R. M. Ziff, "Critical behavior of the susceptible-infectedrecovered model on a square lattice," Phys. Rev. E, vol. 82, p. 051921, 2010.

[45] T. Tomé and M. J. de Oliveira, "Susceptible-infected-recovered and susceptible-exposed-infected models," J. Phys. A, vol. 44, no. 9, p. 095005, 2011.

[46] D. R. de Souza, T. Tomé, and R. M. Ziff, "A new scale-invariant ratio and finite-size scaling for the stochastic susceptible-infected-recovered model," J. Stat. Mec., vol. 2011, no. 03, p. P03006, 2011.

[47] D. Mollison, "Spatial contact models for ecological and epidemic spread," J. R. Stat. Society. Series B (Methodological), vol. 39, no. 3, pp. pp. 283-326, 1977.

[48] T. Antal, M. Droz, A. Lipowski, and G. Ódor, "Critical behavior of a lattice prey-predator model," Phys. Rev. E, vol. 64, p. 036118, 2001.

[49] L. Sander, C. Warren, I. Sokolov, C. Simon, and J. Koopman, "Percolation on heterogeneous networks as a model for epidemics," Math. Biosc., vol. 180 , pp. $293-305,2002$. 
[50] L. Sander, C. Warren, and I. Sokolov, "Epidemics, disorder, and percolation," J. Phys. A, vol. 325, pp. 1 - 8, 2003.

[51] D. R. de Souza, Modelagem de problemas da dinâmica de populações por meio da dinâmica estocástica. (Dissertação de Mestrado, Instituto de Física da Universidade de São Paulo, São Paulo, 2009).

[52] T. E. Harris, "Contact interactions on a lattice.," Ann. Prob., vol. 2, p. 969, 1974.

[53] I. Jensen, "Critical behavior of the three-dimensional contact process," Phys. Rev. A, vol. 45, pp. 563-566, 1992.

[54] J. Chen, "A sirs epidemic model," Appl. Math.- J. Chin. Univ., vol. 19, pp. 101-108, 2004.

[55] Q.-X. Liu, R.-H. Wang, and Z. Jin, "Persistence, extinction and spatio-temporal synchronization of sirs spatial models," J. Stat. Mech., vol. 2009, p. P07007, 2009.

[56] C. E. M. Fernandes, Fundamentos de Física para Geociências - Vol. 1. (Interciência, Rio de Janeiro, 2007).

[57] R. M. Slatt, Stratigraphic Reservoir Characterization for Petroleum Geologist, Geophisicists, and Engineers. (Elsevier, Amsterdam, 2011).

[58] D. Tiab and E. C. Donaldson, Petrophysics: Theory and pratice of measuring reservoir rock and fluid transport properties. (Elsevier, WalthamMA, 2012).

[59] M. B. Isichenko, "Percolation, statistical topography, and transport in random media," Rev. of Mod. Phys, vol. 64, no. 4, pp. 961-1033, 1992.

[60] R. Lenormand, "Capillary fingering: Pecolation and fractal dimension," Transport in Porous Media, vol. 4, pp. 599-612, 1989.

[61] M. Basta, V. Picciarelli, and V. Stella, "An introduction to percolation," Eur. J. Phys., vol. 15, pp. 97-101, 1994.

[62] N. J. Giordano and H. Nakanishi, Computational Physics. (Prentice Hall, New York, 2006).

[63] P. Meakin, Fractals, scaling and growth far from equilibrium. (Cambridge University Press, Cambridge, 1998). 
[64] C. Betrencourt, E. Guyon, and G. Giraud, "Teaching physics out of a bag of marbles," Eur. J. Phys., vol. 1, pp. 206-211, 1980.

[65] J. P. Clerc, G. Giraud, S. Alexander, and E. Guyon, "Conductivity of a mixture of conducting and insulating grains: Dimensionaly effects," Phys. Rev. B, vol. 22, no. 5, pp. 2489-2494, 1980.

[66] B. J. Last and D. J. Thouless, "Percolation theory and eletrical conductivity," Phys. Rev. Lett., vol. 27, no. 25, pp. 2489-2494, 1971.

[67] F. Family and T. Vicsek, Dynamics of Fractal Surfaces. (World Scientific, Singapore, 1991).

[68] H. Ottavi, J. Clerc, G. Giraud, J. Roussenq, E. Guyon, and C. D. Mitescu, "Eletrical conductivity of a mixture of conducting and insulating spheres: an application of some percolation concepts," J. Phys. C: Solid State Phys., vol. 11, p. 056003, 1978.

[69] A. García-García, A. A. Vovk, P. Strichovanec, J. A. Pardo, C. Magén, P. A. Algarabel, J. M. Teresa, L. Morellón, and M. R. Ibarra, "Determination of the percolation threshold in fe/mgo magnectic granular multilayers," J. Phys.: Condens. Matter, vol. 22, p. 056003, 2010.

[70] D. Stauffer, Introduction to Percolation Theory. (Taylor \& Francis, London and Philadelphia, 1995).

[71] D. C. Lorenz and R. M. Ziff, "Precise determination of the bond percolation thresholds and finite-size-scaling corrections for the sc, fcc, and bcc lattices," Phys. Rev. E, vol. 57, no. 1, pp. 230-236, 1998.

[72] M. E. J. Newman and G. T. Barkema, Monte Carlo Methods in Statistical Physics. (Oxford University Press, New York, 2006).

[73] W. Ebeling and I. M. Sokolov, Statistical Thermodynamics and Stochastic Theory of Nonequilibrium Systems. (World Scientific, Singapore, 2005).

[74] M. J. Keeling, "The effects of local spatial structure on epidiological invasions," Proc. R. Soc. London B, vol. 266, 1999.

[75] E. Massad, M. N. Burattini, F. A. B. Coutinho, and L. F. Lopez, "Dengue and the risk of urban yellow fever reintroduction in são paulo state," Rev. Saúde Pública, vol. 37, pp. 477-484, 2003. 
[76] H. Nishiura, "Mathematical and statistical analyses of the spread of dengue," Dengue Bulletin, vol. 30, 2006.

[77] D. A. T. Cummings, S. Iamsirithaworn, J. T. Lessler, A. McDermott, R. Prasanthong, A. Nisalak, R. G. Jarman, D. S. Burke, and R. V. Gibbons, "The impact of the demographic transition on dengue in thailand: Insights from a statistical analysis and mathematical modeling," PLoS Med., vol. 6, no. 9, p. e1000139, 2009.

[78] L. C. d. C. Medeiros, C. A. R. Castilho, C. Braga, W. V. de Souza, L. Regis, and A. M. V. Monteiro, "Modeling the dynamic transmission of dengue fever: Investigating disease persistence," PLoS Negl. Trop. Dis., vol. 5, no. 1, p. e942, 2011.

[79] S. T. R. Pinho, C. P. Ferreira, L. Esteva, F. R. Barreto, V. C. M. e Silva, and M. G. L. Teixeira, "Modelling the dynamics of dengue real epidemics," Phil. Trans. R. Soc., vol. 368, pp. 5679-5693, 2010.

[80] E. O. Agyingi, D. S. Ross, and K. Bathena, "A model of the transmission dynamics of leishmaniasis," J. Biol. Syst., vol. 19, pp. 237-250, 2011.

[81] N. Bacaër and S. Guernaoui, "The epidemic threshold of vectorborne diseases with seasonality: the case of cutaneous leishmaniasis in chichaoua, morocco," J. Math. Biol., vol. 93, pp. 421-436, 2006.

[82] J. Y. Kodaira, Uma abordagem probabilística do número de reprodução básica em modelos epidemiológicos com aplicação na ferrugem do eucalipto. (Dissertação de Mestrado, Instituto de Física de Biociências da Universidade Estadual Paulista, São Paulo, 2011).

[83] E. Bertuzzo, R. Casagrandi, M. Gatto, I. Rodriguez-Iturbe, and A. Rinaldo, "On spatially explicit models of cholera epidemics," Journal of The Royal Society Interface, vol. 7, no. 43, pp. 321-333, 2010.

[84] M. A. M. de Aguiar, E. M. Rauch, and Y. Bar-Yam, "Mean-field approximation to a spatial host-pathogen model," Phys. Rev. E, vol. 67, p. $047102,2003$.

[85] L. B. L. Santos, M. C. Costa, S. T. R. Pinho, R. F. S. Andrade, F. R. Barreto, M. G. Teixeira, and M. L. Barreto, "Periodic forcing in a threelevel cellular automata model for a vector-transmitted disease," Phys. Rev. E, vol. 80, p. 016102, 2009. 
[86] P. Grassberger and A. de la Torre Ann. Phys., vol. 122, pp. 373-396, 1979.

[87] M. A. Muñoz, R. Dickman, A. Vespignani, and S. Zapperi, "Avalanche and spreading exponents in systems with absorbing states," Phys. Rev. E, vol. 59, pp. 6175-6179, 1999.

[88] E. Arashiro and T. Tomé, "The threshold of coexistence and critical behaviour of a predator-prey cellular automaton," Physica A, vol. 40, pp. 887-900, 2007.

[89] F. R. Barreto, M. G. Teixeira, M. C. Costa, M. S. Carvalho, and M. L. Barreto, "Spread pattern of the first dengue epidemic in the city of salvador, brasil," BMC Public Health, vol. 8, pp. 51-71, 2008.

[90] S. Silva, J. Ferreira, and M. Martins, "Epidemic spreading in a scale-free network of regular lattices," Physica A, vol. 377, pp. 689 - 697, 2007.

[91] H. M. Yang, "Epidemiologia da transmissão da dengue," TEMA Tend. Mat. Apl. Comput., vol. 4, pp. 387-396, 2003.

[92] T. Botari, S. G. Alves, and E. D. Leonel, "An epidemiological model for dengue disease using cellular automata," Phys. Rev. E, vol. 83, p. 037101, 2011.

[93] E. A. C. Newton and P. Reiter, "A model of the transmission of dengue fever with evaluation of the impact of ultra-low volume (ulv) insecticide applications on dengue epidemics," Am. J. Trop. Med. Hyg., vol. 47, pp. 709-720, 1992. 